\title{
Recent Trend in \\ Physical Sciences
}

Edited by

Ashutosh Ghosh

Suchandra Chatterjee

Sandeep Poddar

Amiya Bhaumik

Published by :

Lincoln Research and Publications Limited, Australia in collaboration with

Lincoln University College, Malaysia

www.lincolnrpl.org www.lucp.net 


\section{Recent Trend in Physical Sciences}

\section{Edited by}

Ashutosh Ghosh

Vice Chancellor, Rani Rashmoni Green University, Hooghly, West Bengal, India

Suchandra Chatterjee

Associate Professor, Department of Chemistry

Surendranath College, Kolkata, India

Sandeep Poddar

Deputy Vice Chancellor (Research \& Innovation)

Lincoln University College, Malaysia

Amiya Bhaumik

President

Lincoln University College, Malaysia

\section{Published by :}

Lincoln Research and Publications Limited, Australia in collaboration with Lincoln University College, Malaysia 
Copyright (C2021

Lincoln Research and Publications Limited, Australia and Lincoln University College, Malaysia

All rights reserved

No part of this book can be reproduced or transmitted by any means, electronic or mechanical, including photocopying recording or by any information storage and retrieval system without prior written permission from the publisher.

Published on: $30^{\text {th }}$ November 2021

\section{Published by:}

Lincoln Research and Publications Limited

144A, Marsden Road

Ermington, Sydney

NSW 2115

Australia

Tel.: +61-411497511

E-mail: info@lincolnrpl.org

Web.: www.lincolnrpl.org

in collaboration with

\section{Lincoln University College}

Wisma Lincoln

No. 12-18, Off Jalan, Perbandaran SS 6/12

47301 Petaling Jaya

Selangor Darul Ehsan

Malaysia

Tel.: +603-7806 3478

Fax: +603-7806 3479

Toll Free: 1-300-880-111

E-mail: lucp@lincoln.edu.my

Web.: www.lucp.net

ISBN: 978-0-6488798-5-5

eISBN 978-967-2819-04-2

doi:10.31674/book.2021.rtps

Price: AUD 50 


\section{Editors}

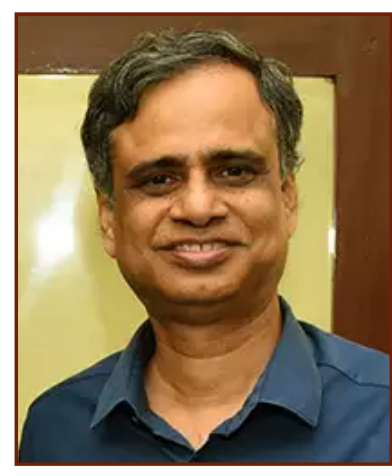

\section{Ashutosh Ghosh}

Vice Chancellor

Rani Rashmoni Green University, Hooghly, WestBengal, India

Professor Ashutosh Ghosh is currently acting as the Vice Chancellorof Rani Rashmoni Green University, Hooghly, West Bengal.After graduation and post-graduation in Chemistry from Presidency college, Kolkata and University of Calcutta respectively, he completed his Ph.D. from Indian Association for the Cultivation of Science, Kolkata. His field of research is synthesis, structural characterization, magnetic properties, and catalytic activities of homoand hetero-metallic poly-nuclear complexes of 1 st transition metal ions. Under his supervision till date 25 students have been awarded with Ph.D. and he has 290 published papers in various international journals to his credit. He had been UNESCO Fellow at Charles University, Czechoslovakia; MONBUSHO Fellow at Nagoya University, Japan; JSPS Fellow at Tsukuba University, Japan and Visiting Scientist at University of Utah, USA and University of Dusseldorf, Germany. He is elected fellow of both Indian Academy of Science (Bangalore) and West Bengal Academy of Science and Technology and recipient of several prestigious awards like Rheometric Scientific-ITAS Award (1995), CRSI Bronze Medal (2016), Professor Priyadaranjan Ray Memorial Award (2017) of Indian Chemical Society and ACT Life-Time Achievement Award (2020) by Association of Chemistry Teachers.

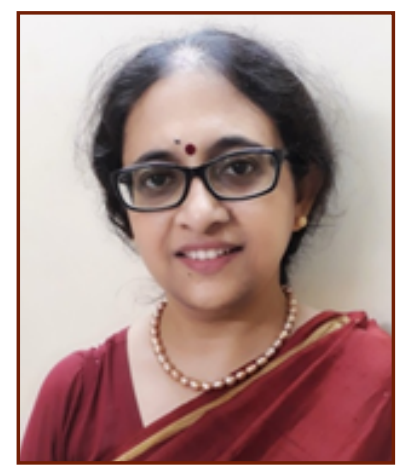

\section{Suchandra Chatterjee}

Associate Professor, Department of Chemistry

Surendranath College, Kolkata, India

After completing her graduation from Presidency College, Kolkata with Honours in Chemistry, Suchandra did her masters from University of Calcutta with specialization in Physical Chemistry, followed by her doctoral research work at Saha Institute of Nuclear Physics, Kolkata on Photochemistry and Molecular Spectroscopy as a CSIR Fellow. She has publications in several journals of international repute. 2001 onwards she is till date effectively occupied with teaching, first as Lecturer and Senior Lecturer of Physical Chemistry at Serampore College, Hooghly, trailed by her current role as an Associate Professor at Surendranath College, Kolkata. She is a recipient of Ranit Pratibha Puraskar for Advancement of Chemistry and an elected fellow of Indian Chemical Society. 


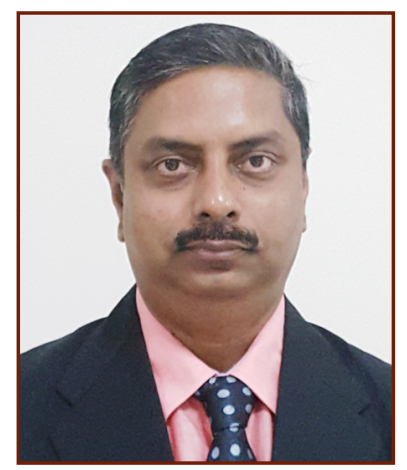

\section{Sandeep Poddar} Deputy Vice Chancellor (Research \& Innovation) Lincoln University College, Malaysia

Prof. Dr. Sandeep Poddar, presently the Deputy Vice Chancellor (Research \& Innovation) of Lincoln University College, Malaysia. He also served as Senior Research Director and Executive Editor(Publications), Lincoln University College, Adjunct Faculty (Honorary), Bharat Center Canada. He has graduated from University of Calcutta in 1993 with Honours in Zoology, he has obtained Post Graduate Diploma in Dietetics from All India Institute of Hygiene and Public Health 1995, Master of Science in Zoology with specialization in Biochemical Genetics from Dayalbagh Educational Institute 1998 with distinction. In addition to this he also obtained Master of Business Administration (MBA) from Lincoln University College in 2021. He has completed Ph.D. in Zoology from Vivekananda Institute of Medical Sciences on Cytotoxicity in 2004. After completing Ph.D. he pursued Post Doctoral Research in different projects on Hemoglobinopathies and Oral Cancer mutation. He is serving as reviewer of several International Journals. He has published several research papers, organized international conferences, and edited books in Malaysia, Australia and India. Dr. Sandeep is founder Assistant Secretary of Dr. Tarak Nath Podder Memorial Foundation, Kolkata, India.

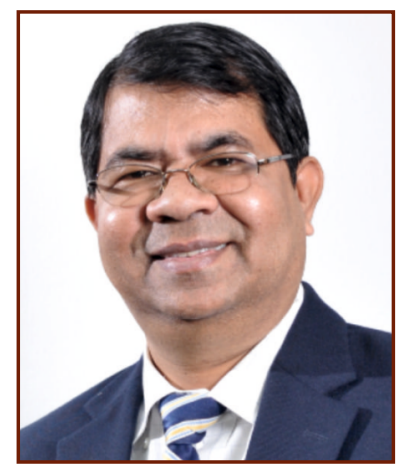

\section{Amiya Bhaumik}

\section{President}

Lincoln University College, Malaysia

Dr. Amiya Bhaumik is the Founder and Former Vice-Chancellor of Lincoln University College. He is purely from the field of education. Dr. Bhaumik is Executive Vice President of the International Education Consulting Group, St. Louis, USA since 1999. Dr. Amiya Bhaumik was Research Fellow of UNESCO, Paris. During this tenure, Dr. Bhaumik has traveled extensively to Europe, Africa, Asia and Latin America. He has served as Professor of Business Administration in University of Lucknow, India and in University of Malaya and many other places. Dr. Amiya Bhaumik is a very dynamic personality. He has authored numerous book chapters and has huge number of publication in many national and international journals. He has also edited several books. 
Contents

Pages

Editorial

i-ii

Foreword

iii

Photocatalyst- Chataracterization and Application in Photochemical Water Splitting

Ayan Sarkar, Sounok Sen, Abishek Kumar Jha, Sourav Misra

Kaushik Bhagat, Harisadhan Ghosh

Synthesis, Characterization and Structure Modelling of Graphene Oxide

Shashi Kant Singh, Hari Shankar Biswas

A Brief Idea About Protein-Protein Interactions Modulators

Saumik Mal, Boijoyanta Howlader, Apurba Biswas

Metal Organic Framework: A Future Tool for Nano-Catalysis and Drug Delivery

Prince Saha, Srijita Bhowmik, Supriya Mondal, Suchandra Chatterjee

Artificial Molecular Machines with Bio-inspired Mechanisms of Natural Molecular

Devices: An Overview

Arghadeep Sarkar, Suchandra Chatterjee

Variational Monte Carlo Approach for Solving Quantum Mechanical Systems

$47-54$

Rohit Kumar Thapa, Subha Pritam Pal, Soumyadeeep Mandal, Sourav Mitra

A Review on the Phenomenological Theory of Phase Transitions

Argha Deb, Animesh Mallick, Avijit Sen Majumder, Deepanwita Das, Eram Tanwir, Asok

Kumar Das

Characteristics of the Detectors for Nuclear Radiation

Indranil Sadhya, Jamal Uddin, Mihir Kumar Das, Naved Akram, Nilay Sankar Chattopadhyay,

Prasanna Kumar Mondal

Optical Phase Locked Loop Advancement

Poushali Mallick, Pritam Mandal, Rahul Paul, Santanu Moi, Purnendu Prakas Pal

Coulomb Blockade Effect in the Context of Set

$79-81$

Rajibul Islam, Rajdeep Saha, Snehasis Mondal, Ranjit Maity

Electromagnetically Induced Transparency in Coherent Atomic Media

Arin Bhattacharya, Abhasita Das Munshi, Abhiroop Ghosh, Anand Singh, Aparajita Das

Limitations of MOSFET Scaling and its Solution

$92-95$

Ishan Saha, Mohammad Javed Imam, Bonsari Choudhury, Haridas Kanji, Budhaditya Das,

Emona Datta

A Comprehensive Study of the Past, Present and Future of Solid-state Drive

96-98

Subhrangshu Hait, Shib Shankar Biswas 



\section{Editorial}

Physical sciences help us to unearth the fundamental laws of nature. It is a collective term that deals with astronomy, chemistry, materials science and physics. The goal of this book are to develop a better understanding of physics research and its applications to promote new industries; to innovate knowledge about recent breakthroughs in physics, both fundamental and technological aspects; and to implement international cooperation in new trends in physics research and to improve the performance of physics research facilities.

Photocatalysts are one of the important research fields in modern material chemistry. Photocatalysts can catalyse chemical reactions with the help of radiations (mainly solar). These are compounds which can affect the rate of a chemical process by absorbing a certain frequency of light (or photon). They are generally semiconductor materials which have a certain band gap.

Brominated organic compounds are highly important because of their tremendous use in the production of pharmaceuticals, agrochemicals, dyes, UV sunscreens, high performance polymers etc. Various brominated marine natural products have drawn a great pharmacological interest. On the other hand, organic bromides are quite useful synthons for important chemical transformations such as substitution and cross coupling reactions.

Graphene Oxide (GO), being an important derivative of graphene, has drawn a wide attention from the researchers worldwide. Several innovative methods have been developed today for the synthesis of graphene oxide. However, the large-scale production is highly important for the industrial as well as other commercial applications.

Protein-protein Interaction (PPIs) play an important role in the life cycle. Many studies have suggested that aberrant PPIs relate to several fatal diseases including cancer, infectious diseases and also neurodegenerative diseases, regulating diseases. Consequently, PPIs, usually small molecule inhibitors, are an important diagnostic strategy for disease progression as well as a crucial technique for medication development.

Metal Organic Frameworks or MOFs are organic-inorganic combined porous and permeable substances. They are made up of metallic ions and organic ligands. Ligands are ions/molecules that are connected to the metal through coordination bonds. During the last two decades MOFs have acquired huge consideration because of their different attributes including high porosity, large surface area, controllable pore sizes, and so on. Current review presents a comprehensive study on different methodologies for synthesis of those MOFs that have higher capacity, rigidity, durability, and ecofriendly nature.

A molecular machine is defined as an assembly of molecular components that are interlocked to bring certain movement or conversion of energy. These machines can be controlled by making alteration in the external environment. A molecular machine, also known as a nanite or nanomachine, is a molecular component that responds to certain stimuli by producing quasi-mechanical movements (output) (input). Macromolecular machines are often used in cellular biology to conduct functions that are critical to life, such as DNA replication and ATP generation.

Complex quantum mechanical systems cannot be solved accurately using analytic methods and must be solved numerically. The variational Monte Carlo approach is one of such techniques to simulate physical systems numerically and is widely used to perform ab initio calculations on the system.

Phase transitions are ubiquitous around us. From water boiling in a saucepan kept on a stove to cosmological phase transitions in the early universe, phase transitions play a very important role in the world around us. A phase transition is essentially associated with change in the symmetry properties of the system undergoing phase transition.

The radiation characterization technique has undergone rapid transformation over the years. It is well known that the ionizing radiation (such as cosmic rays, natural radioactive materials etc.) has always been present around us. One of the most useful detectors is scintillation counter which uses the light 
emitted when charged particles pass through certain materials. The basic process here is excitation. Other type of detectors makes use of ionization produced by charged particles passing through the material.

Optical Phase Locked Loops are expected to have a wide range of applications in fibre optic communication networks. Understanding of phase lock theories requires knowledge of several disciplines of engineering mathematics, nonlinear mechanics, control theory, Fourier transform, and Laplace transform of functions.

As we measure materials down to nanoscale, some interesting properties and phenomenon start to emerge that are not so obvious at macroscale, and sometimes completely different from its bulk properties. One such phenomenon is Coulomb Blockade (CB) which arises due to, in layman terms, Coulomb repulsion. As we make a device smaller, its capacitance decreases and in turn its charging energy, which is inversely proportional to its capacitance, increases.

Quantum interference occurs between the optical transition pathways when the quantum states of atoms or molecules are coherently prepared by lasers. It can dramatically modify the optical responses of an atomic medium to the weak probe field in presence of a strong pump field. Electromagnetically induced transparency (EIT) in atomic medium is the phenomenon where an initially absorbing atomic medium becomes transparent to a weak probe field (typically lasers) in the presence of a strong control field (laser field).

Over the past decades, scaling of complementary metal-oxide-semiconductor (CMOS) technology is a main factor for constant improvement in semiconductor industry. However, because of downscaling, when the device dimensions reduce to nanometre range, CMOS devices face lots of severe problems. To maintain the improvements, various materials and advanced device structures had been introduced.

Technology plays an important role in a changing world and the world changes with the change in technology. In the past data was saved to floppy disks or external drives because the internal hard disk drives used in computers in the market at that time were very expensive. Scientists and engineers have been putting their efforts for years to prepare data storage more concise, speedy, reputable, less power consumption and accessible. Due to the vast changes occurring in the world, new technology has brought about remarkable transformations and one of them is flash memory which is known for its renowned application the solid-state disk or solid state derive (SSD).

Physical sciences have played and continue to play an important part in the advancement and development of nations and communities in the contemporary period. Physical sciences are currently promoting new businesses and innovating information about recent breakthroughs in physics, both basic and technical.

The editors would like to thank all the authors for their contribution in such varied topics that will help to create awareness in various aspect of life. They are also thankful to the Principal, Surendranath College, Kolkata, India and the Management of Lincoln University College, Malaysia along with Lincoln Research \& Publishing Limited, Australia for giving them the necessary permission to publish this book. We are also thankful to Department of Biotechnology, Government of India for providing the financial assistance. The objective of the book is to develop greater understanding of research in the field of research and its applications to encourage new industries; to innovate knowledge about recent advances in physics, both the fundamental and technological aspects; to implement international cooperation in new trends in physics research and to improve the performance of the physics research facilities all over the world.

\author{
Ashutosh Ghosh \\ Suchandra Chatterjee \\ Sandeep Poddar \\ Amiya Bhaumik
}




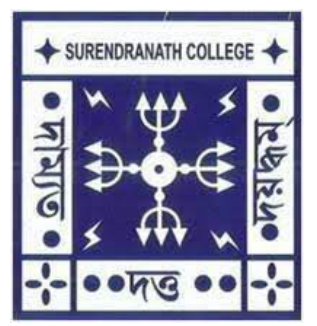

\title{
Surendranath College
}

24/2, Mahatma Gandhi Road, Kolkata - 700 009, India

Phone: (033) 2350 2864, 2354 3876, Fax: (033) 23502864

E-mail: snlcollege@gmail.com, Web.: www.snlawcollege.ac.in

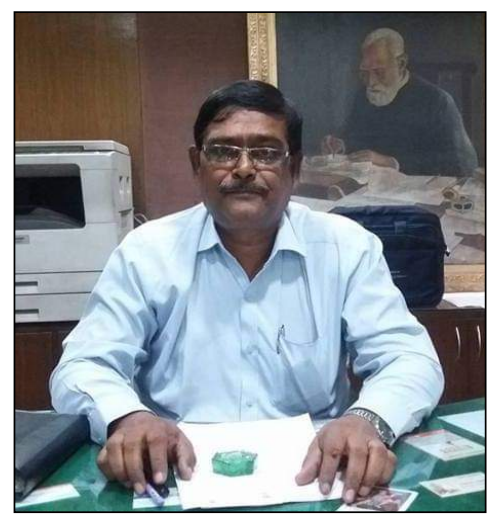

\author{
Dr. Indranil Kar \\ M.Sc., Ph.D. \\ Principal \\ Surendranath College \\ Kolkata, India
}

\section{FOREWORD}

It is my great pleasure to introduce this book titled as "Recent Trend in Physical Sciences," a collection of review articles written by the undergraduate students of the four DBT STAR departments of Surendranath College, Kolkata, covering a wide range of contemporary themes on current development in various areas of physical sciences, under able mentorship of their faculty members as mentors.

The volume consists of fourteen chapters and pursues specific objectives while complementing the existing literature on different areas of Physical and Chemical Sciences. It explores the scope of future developement in various related emerging fields and analyses the challenges in doing so. I extend special thanks and underline my deepest appreciation to the publisher for publishing this issue, Department of Biotechnology, Government of India for providing the financial assistance and all the editors of this book for their real hard work and sincere effort in bringing out this wonderful volume.

While I hope this collection will have particular appeal to all the new young readers in this field, I am confident that the volume will raise interest among our regular readership of scholars and practitioners elsewhere as well, thanks to the relevance and diversity of contributions compiled here in this volume.

Happy reading to all!

Dr. Indranil Kar

Principal

Surendranath College

Kolkata, India 



\title{
Photocatalyst- Chataracterization and Application in Photochemical Water Splitting
}

\author{
Ayan Sarkar, Sounok Sen, Abishek Kumar Jha, Sourav Misra* \\ Department of Chemistry, Surendranath College, Kolkata, India \\ *Corresponding Author's Email: souravmisra.exam@gmail.com
}

\begin{abstract}
Photocatalysts are one of the important research fields in modern material chemistry. Photocatalysts can catalyze chemical reactions with the help of radiations (mainly solar). In this review article, some common characterization techniques (Raman spectrum, SEM, TEM etc) of photacatalytic materials are discussed in brief. The applications of photocatalytic materials are now widespread. Here we have mentioned two important photocatalytic materials $-\mathrm{TiO}_{2}$ and $\left[\mathrm{Ru}(\mathrm{bpy})_{3}\right]^{2}+$ complexes. Their mode of application in water splitting using solar radiation are discussed here in brief.
\end{abstract}

Keywords: SEM, TEM, Photochemical Water Splitting, Band Gap, Titanium Dioxide, Ruthenium -Bipyridyl Complexes

\section{INTRODUCTION}

Photocatalysts are compounds which can affect the rate of a chemical process by absorbing a certain frequency of light (or photon). The best example of a natural photocatalyst is chlorophyll which absorbs solar radiation and catalyze in the splitting of water to produce oxygen during photosynthesis in green plants. Photocatalysts are generally semiconductor materials which have a certain band gap. When the energy value of this band gap becomes comparable with that of certain radiation (mostly solar); energy get absorbed by the semiconductor and that semiconducting material get excited. This excited semiconductor can then behave as a photocatalyst and catalyze the reaction under consideration (Martin, 2015).

\section{DISCUSSION}

\section{Characterization Methods for Photocatalysts}

A huge variety of methods for the characterization for photocatalytic materials are available nowadays. These methods not only help us to confirm results, but also are very much useful to understand the principle of workings of a photocatalyst (Gilreath, 1985).

\section{UV-Visible Spectroscopy}

The absorption spectra of a material are observed using this method. UV-Visible spectrophotometers ('UV-Vis') can measure the electromagnetic spectrum from ultraviolet to visible wavelengths. Because semiconductors have discrete energy levels, quantum-mechanically allowed electronic transitions can occur within these energy levels, molecules frequently undergo electronic transitions at these frequencies. As a result, we can determine the semiconductor's band gap size and type.

\section{Scanning and Transmission Electron Microscopy}

The surface structure of semiconductors is imaged using two separate techniques: scanning electron microscopy (SEM) and transmission electron microscopy (TEM).

SEM is frequently used to determine particle size and analyze surface morphology, particularly with thick materials. The SEM generates a high-energy electron beam that interacts with the sample's surface, resulting in secondary electrons, backscattered electrons, and diffracted backscattered electrons. The detectors capture all these electrons, which are then converted into a high-depth-of-field 
image. Although it is a two-dimensional image, this gives it a three-dimensional aspect.

The transmission of an electron beam through a thin material (less than $100 \mathrm{~nm}$ ) is the subject of TEM. The beam interacts with the sample, causing it to refract around the atoms. This produces a photograph on photographic film or a CCD. It's used to image thin films or nanoparticles that are smaller than $10 \mathrm{~nm}$ in size. It can also be used to detect nano-scale surface dopants, such as metallic or crystalline components, that are difficult to see on a SEM picture. Unlike SEM, TEM permits non-conductive sampling to be investigated. When a SEM is used to examine a non-conductive sample, a phenomenon known as 'charging' occurs. This charging effect frequently damages the sample, making imaging extremely difficult.

Transmission and Scanning EDX is a feature that is typically included with electron microscopes (Energy Dispersive X-ray Spectroscopy, abbreviated as EDS). This kind of analysis is effective for determining the elements in samples. It produces a spectrum of elements and their proportions in the specimen under inquiry. The SEM's electron beam is used to excite the electrons in the sample's ground state and compel them to be expelled. The empty ground state is subsequently filled by an electron from any higher energy shell, resulting in the emission of an X-ray. The energy of the emitted X-ray is equal to the difference between the two shells, making it unique to individual elements. This can be utilized in photocatalysis research to determine the extent of co-catalyst distribution on the catalyst surface. It can also be used to find contaminants.

\section{Raman Spectroscopy}

Raman spectroscopy is a technique for finding vibrational, rotational, and other low frequency modes in a compound that is less widely used. A monochromatic light source illuminates the sample in Raman spectroscopy, which is then scattered by the substance under analysis. An input photon polarizes the electron density or electron cloud, resulting in an unstable virtual state that is stabilized by the reemission of another photon of comparable, but not identical, energy. The amount of electron cloud deformation (polarizability) caused by the photon in relation to the vibrational mode coordinates is required for an atom to produce the Raman effect (refer to figure 1).

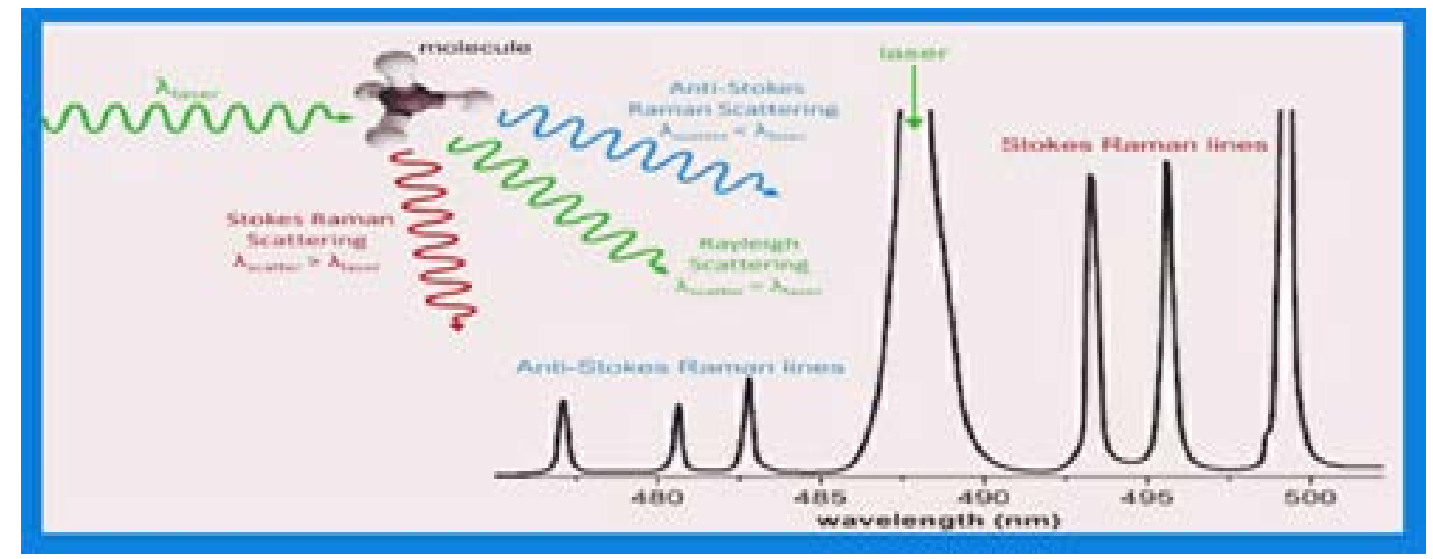

Figure 1: Raman Spectroscopy analyzing Semiconductor Photocatalysts

The intensity of Raman scattering is directly proportional to the magnitude of polarizability. This allows Raman spectroscopy to investigate the various vibrational or rotational transitions that are disallowed in centrosymmetric molecules according to the mutual exclusion condition. The plot, which shows Raman shift (cm1) versus intensity, shows which vibrational or rotational bands are present in the molecule or material.

Semiconductor photocatalysts can be studied using Raman spectroscopy. Identifying probable impurities on the surface of the semiconductor employed as a photocatalyst and correlating their photocatalytic activity is extremely beneficial. 


\section{Photocatalytic Water Splitting}

Solar water splitting using an inorganic semiconductor is one of the most environmentally beneficial methods for producing clean, renewable fuels like hydrogen from abundant natural resources. TiO2 and $[\mathrm{Ru}(\mathrm{bpy}) 3] 3+$ complexes are two of the most prominent photocatalysts in this regard.

\section{Fundamentals of water splitting}

Water electrolysis is a well-studied procedure. This process can result in the production of hydrogen. It does, however, necessitate a tremendous amount of energy. The use of solar radiation is used to avoid this large energy input while maintaining hydrogen production. Water photolysis is a technique that uses a certain frequency of radiation to enhance charge carrier separation in a semiconductor. Water splits as a result of a photo-generated voltage (refer to figure 2 ).

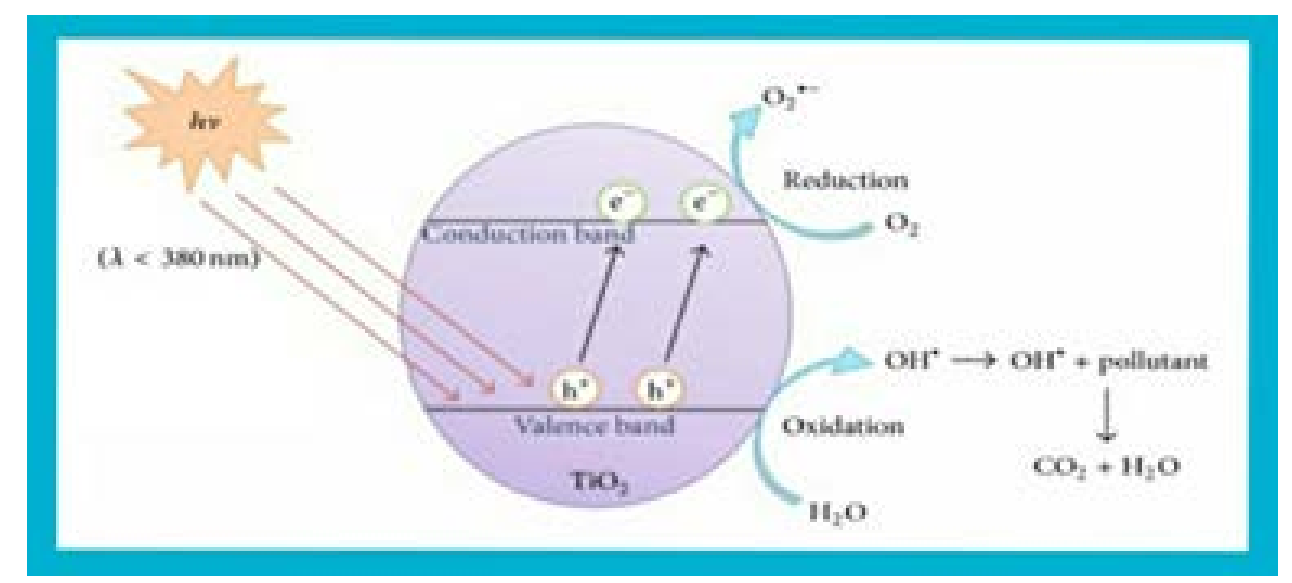

Figure 2: General Reaction for Water Splitting

General reaction for water splitting

$$
2 \mathrm{H}_{2} \mathrm{O} \rightarrow \mathrm{O}_{2}+4 \mathrm{H}^{+}+4 \mathrm{e}^{-} \quad 4 \mathrm{H}^{+}+4 \mathrm{e}^{-} \rightarrow 2 \mathrm{H}_{2}
$$

As most of us are aware, the earliest way of conventional water splitting used a titanium anode and a platinum cathode, with the response being driven by an electrical bias. As a result, this structure can be regarded as a photo-electrochemical cell.

When hydrogen and oxygen are together in a confined space, they tend to explode. Because of this, when using a suspension framework and an effective photocatalyst for the process of water splitting, safety precautions must be observed. Water splitting on a larger mechanical scale using colloidal frameworks may be illogical since combustibility cutoff points may be reached unless the framework is made more unexpected by using compressors and separating units to transport hydrogen and oxygen blends from reactors. Suspension frameworks are a feasible technique for lab-scale materials testing, especially for a half reaction.

If a substance is discovered to be very efficient, it can be integrated into a PEC (Photo Electrochemical Cell) system, with hydrogen and oxygen produced in distinct compartments of the same cell. Electron acceptors and donors are compounds with high relative electronegativity (for electron acceptors/scavengers) or electro positivity (for electron donors/hole scavengers) that can stop the production of hydrogen (electron donor) or oxygen (electron acceptor) by combining with the respective charge carrier. Scavenger chemicals must also be highly soluble in water in order to readily give or receive charge.

$\mathrm{S} 2 \mathrm{O} 82, \mathrm{Ce}(\mathrm{SO} 4) 2, \mathrm{FeCl} 3$, and other electron acceptors are examples. Ag+ from AgNO3 is the most common electron acceptor because it has been found to scavenge electrons more efficiently than any other electron acceptor. Ethanol, methanol, and triethanolamine are the most commonly utilised electron donors (TEOA) (refer to figure 3). 


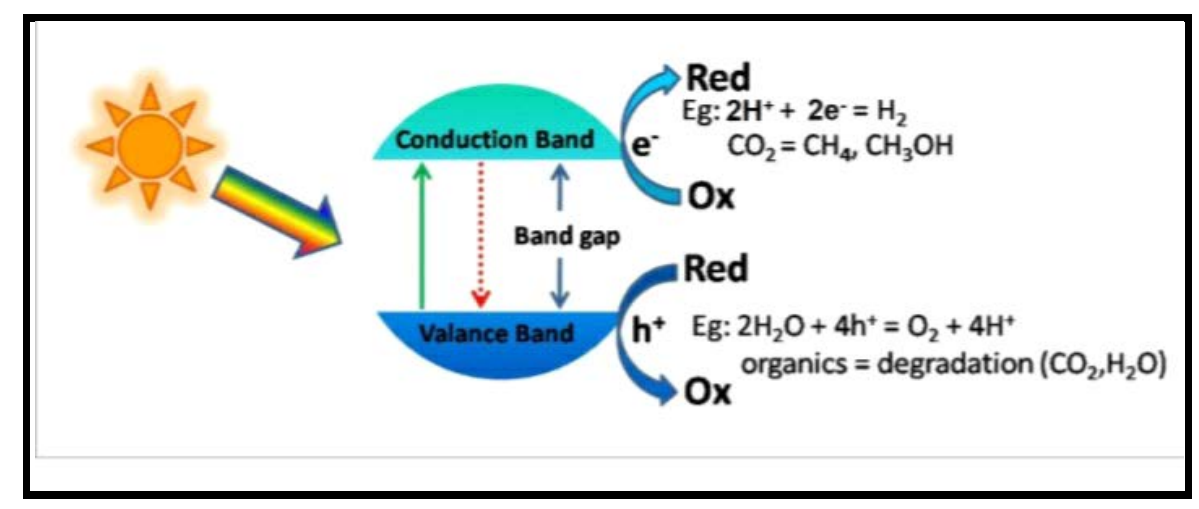

Figure 3: Electron acceptors

Photochemical water splitting by $\mathrm{TiO}_{2}$

Several methods of preparation are used for the synthesis of $\mathrm{TiO}_{2}$ materials. The synthesis procedure depends on the final application of the material. The methods can be classified into thermal reactions, deposition methods, sol-gel, Micelle, and electromagnetic methods. But mostly doped $\mathrm{TiO}_{2}$ are better candidates for water splitting. This doping on $\mathrm{TiO}_{2}$ can be of two types- metal doping and non-metal doping.

Atanelov, Gruber \& Mohn (2015) doped rutile $\mathrm{TiO}_{2}$ with $\mathrm{C}$ and it seems to have lesser photocatalytic performances than pure $\mathrm{TiO}_{2}$, this is due to $\mathrm{C}-\mathrm{O}$ dimes creating some mid-band states. However, if $\mathrm{N}$ doped rutile TiO2shows no mid-bandgap states. Ghuman \& Singh (2013) used Rh to dope rutile $\mathrm{TiO}_{2}$ and found that it had a lower bandgap but with more recombination centers. When they used $\mathrm{Rh}-\mathrm{Nb}$ as co-dopants on rutile $\mathrm{TiO}_{2}$, they obtained no isolated band-gap states that might act as a recombination center. Moreover, the bandgap was decreased by $0.5 \mathrm{eV}$, which makes it a better photocatalyst.

The mechanism of $\mathrm{TiO}_{2}$ as photocatalyst can be shown by the following diagram (figure 4):

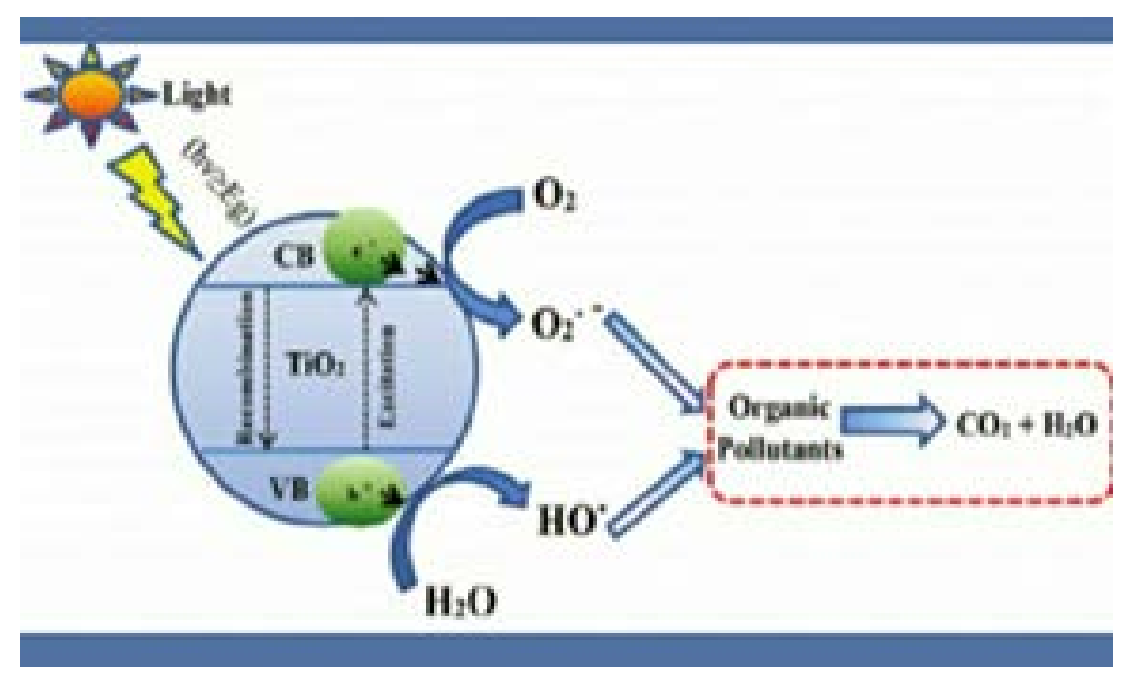

Figure 4: Mechanism of $\mathrm{TiO}_{2}$

\section{Photochemical water splitting by $[\mathrm{Ru}(\mathrm{bpy}) 3]^{2}+$ complex}

$\mathrm{Ru}(\mathrm{bpy})_{3}{ }^{2}+$ can catalyze the splitting of water to generate the fuel gas $\left(\mathrm{H}_{2}\right.$ and $\left.\mathrm{O}_{2}\right)$ which is used in fuel cell to produce electricity. $\mathrm{Ru}(\mathrm{bpy})_{3}{ }^{2}+$ absorbs photons $(\lambda=452 \mathrm{~nm})$ and is excited to singlet $\left[R u(b p y) 3_{3}{ }^{2}\right]^{* *}$ which relaxes to the relatively long- lived triplet $\left[R u(b p y){ }_{3}{ }^{2}\right]^{*}$ species (Juris et al. 1988).

In photo-excitation process of $\mathrm{Ru}(\mathrm{II})\left(\mathrm{d}^{6}\right)$, one electron from $\mathrm{t}_{2 \mathrm{~g}}$ orbital $(\mathrm{HOMO})$ of $\mathrm{Ru}$ is excited to empty $\Pi^{*}$ orbital (LUMO) of bpy. 


$$
\begin{aligned}
& {\left[\mathrm{Ru}(\mathrm{bpy}) 3^{2+}\right]^{*}+\mathrm{e} \rightarrow\left[\mathrm{Ru}(\mathrm{bpy}) 3^{+}\right]} \\
& \mathrm{E}^{0}=0.84 \mathrm{~V} \\
& 2 \mathrm{H}_{2} \mathrm{O}+2 \mathrm{e} \rightarrow \mathrm{H}_{2}+2 \mathrm{OH}^{-} \\
& \mathrm{E}^{0}=-0.41 \mathrm{~V} \\
& {[\mathrm{Ru}(\mathrm{bpy}) 3]^{3+}+\mathrm{e} \rightarrow\left[\mathrm{Ru}(\mathrm{bpy}) 3^{2+}\right]^{*}} \\
& \mathrm{E}^{0}=-0.86 \mathrm{~V} \\
& 4 \mathrm{H}^{+}+\mathrm{O}_{2}+4 \mathrm{e} \rightarrow 2 \mathrm{H}_{2} \mathrm{O} \\
& \mathrm{E}^{0}=-0.82 \mathrm{~V}
\end{aligned}
$$

In the long- lived triplet intermediate, $\left[\mathrm{Ru}(\mathrm{bpy}) 3^{2}+\right]^{*}$ an electron from $\mathrm{ABMO}$ of bpy ligand can be easily donated to an oxidant and this makes the $\left[\mathrm{Ru}(\mathrm{bpy}) 3_{3}{ }^{2}\right]^{*}$ a better reducing agent. On the other hand, vacancy created at $t_{2 g}$ level of Ru- center makes it electron-greedy and thus $\left[R u(b p y){ }_{3}{ }^{2}\right]^{*}$ can also act as a better oxidizing agent. The splitting of water by $\left[\mathrm{Ru}(\mathrm{bpy}) 3^{2}{ }^{2}\right]^{*}$ is thermodynamically feasible(as seen from reduction potential values). But there are some kinetic barriers which prevents direct use of $\left[\mathrm{Ru}(\mathrm{bpy}) 3^{2}+\right]^{*}$

$\mathrm{Ru}$ - complexes can act as 1 e transfer reagents while for photochemical splitting of water requires $2 \mathrm{e}$ and $4 \mathrm{e}$ transfer. Hence, before e transfer, the excited state may spontaneously decay (Porter, 1983). That's why during photolysis of water, different quenching agents are required to stabilize the $\left[\mathrm{Ru}(\mathrm{bpy}) 3^{2+}\right]^{*}$ and intermediate redox species. One such suggested quenching agent is methyl viologen $\left(\mathrm{MV}^{2+}\right)$ (refer to figure 5).

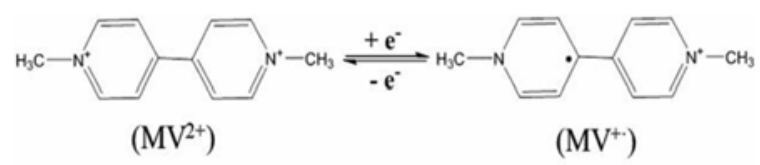

Figure 5: Methyl Viologen (Mv2+)- A Quenching Agent

The Ru- complex harvests the solar radiation through photochemical excitation and then the quencher $\left(\mathrm{Mv}^{2}+\right)$ sets up a redox reaction leading to decomposition of water. The redox reaction is catalyzed by finely divided $\mathrm{Pt}$ and $\mathrm{RuO}_{2}$ supported on $\mathrm{TiO}_{2}$. To regenerate $\mathrm{Ru}(\mathrm{bpy})_{3}{ }^{2}+$ from $\mathrm{Ru}(\mathrm{bpy}) 33+$ triethanolamine (TEA) may be added. The schematic representation of the catalytic activity of $\left[\mathrm{Ru}(\mathrm{bpy}) 3^{2}+\right]$ complex in photochemical water splitting is given below in figure 6 .

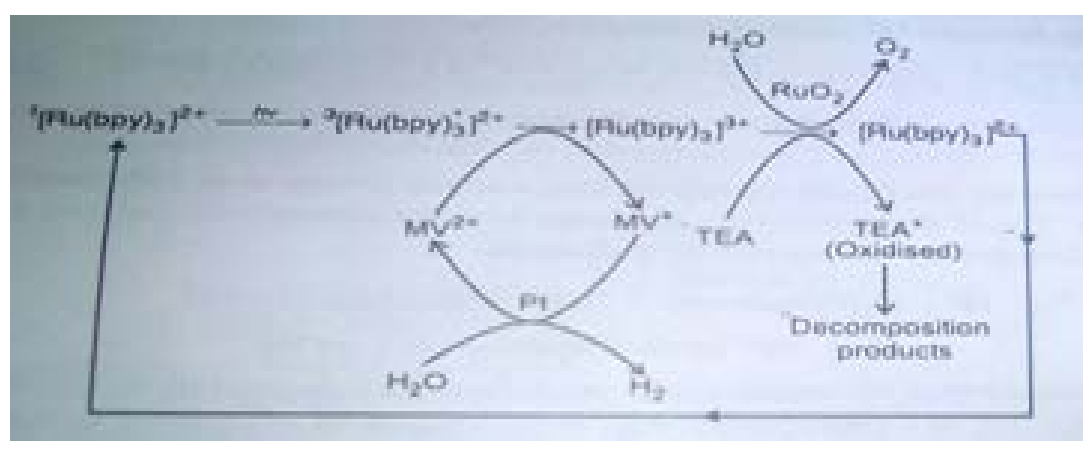

Figure 6: Schematic representation of the catalytic activity of $\left[R u(b p y)_{3}{ }^{2}+\right]$ complex in photochemical water splitting

\section{Calculation of efficiency}

Numerous strategies and conditions can be utilized to figure the productivity of a semiconductor's capacity to decay water. Ordinarily, the creation of hydrogen or oxygen is estimated by the unit micromole of item each hour, per gram of photocatalyst; ( $\mu \mathrm{mol} \mathrm{hr}-1 \mathrm{~g}-1)$. Despite being worthy unit for introducing rates, it is unacceptable for deciding effectiveness, on the grounds that the rate of evaluation of most gases rely upon the exploratory conditions: source of the light, size of the reactor, quantity of catalyst etc. This makes evaluation rates somewhat exceptional.

The number of the incident photons can be determined utilizing a thermopile or silicon identifier/photodiode. Sadly, the quantity of consumed photons (by the photocatalyst) is often 
significantly lower than that of light incident upon it because of scattering and transmission. In any case, the amount of catalyst is to be balanced in such a way that it can minimize the loss due to reflection and can adjust with variable dispersity.

In this manner, it is feasible to utilize an experimentation cycle, whereby concentration of catalyst is changed, and afterward is compared with the concentration of the products (in - $\mu \mathrm{mol} \mathrm{hr}-1 \mathrm{~g}-1$.)

\section{CONCLUSION}

Therefore, Photocatalysts are defined as semiconductor materials with a certain band gap width. When the energy value of this band gap matches that of radiation (mainly solar), the semiconductor absorbs the energy, and the semiconducting material becomes activated. Photocatalytic materials are being used in a wide range of applications. Two major photocatalytic materials are mentioned here: $\mathrm{TiO}_{2}$ and $[\mathrm{Ru}(\mathrm{bpy}) 3] 2+$ complexes. Their mode of application in solar-powered water splitting is also briefly explored here in this study.

\section{REFERENCES}

Atanelov, J., Gruber, C., \& Mohn, P. (2015). The electronic and magnetic structure of p-element (C, N) doped rutile-TiO2; a hybrid DFT study. Computational Materials Science, 98, 42-50.

Ghuman, K. K., \& Singh, C. V. (2013). A DFT+ U study of (Rh, Nb)-codoped rutile TiO2. Journal of Physics: Condensed Matter, 25(8), 085501.

Gilreath, E. S. (1958). Fundamental concepts of inorganic chemistry. McGraw-Hill.

Juris, A., Campagna, S., Balzani, V., Gremaud, G., \& Von Zelewsky, A. (1988). Absorption spectra, luminescence properties, and electrochemical behavior of tris-heteroleptic ruthenium (II) polypyridine complexes. Inorganic Chemistry, 27(20), 3652-3655.

Martin, D. J. (2015). Investigation into high efficiency visible light photocatalysts for water reduction and oxidation. Springer.

Porter, G. B. (1983). Introduction to inorganic photochemistry: Principles and methods. 


\title{
1,3-Dibromo-5,5-Dimethyl Hydantoin (DBDMH): A Useful Brominating Reagent In Organic Synthesis
}

\author{
Kaushik Bhagat, Harisadhan Ghosh* \\ Department of Chemistry, Surendranath College, Kolkata, India \\ ${ }^{*}$ Corresponding Author's Email: ghosh.harisadhan@gmail.com
}

\begin{abstract}
The application of 1,3-dibromo-5,5-dimethyl hydantoin (DBDMH) in various organic synthesis has attracted a great deal of attention nowadays. This is due to its low cost, higher bromine content, lowtoxicity issues, easy handling. It has been used both as a catalyst as well as a reagent. In this book chapter, the use of DBDMH as a brominating reagent is summarised. Various stereoselective, regioselective and enantioselective bromination reaction has been discussed where DBDMH has been used as the most suitable brominating reagent. A special emphasis is devoted for the documentation of aromatic bromination mediated by DBDMH.
\end{abstract}

\section{Keywords: N-Bromo Compounds; Bromination; DBDMH; Aromatic Bromination; Regioselectivity}

\section{INTRODUCTION}

Brominated organic compounds are highly important because of their tremendous use in the production of pharmaceuticals, agrochemicals, dyes, UV sunscreens, high performance polymers etc. Various brominated marine natural products have drawn a great pharmacological interest. On the other hand, organic bromides are quite useful synthons for important chemical transformations such as substitution and cross coupling reactions (Desmurs, Goldstein \& Gérard, 1995).

To synthesize the brominated organic compounds usually the molecular bromine $\left(\mathrm{Br}_{2}\right)$ is used. But the major problem of using the elemental bromine is its handling difficulties in laboratory, as it is hazardous and is a strong irritant. That is why, the use and handling of bromine needs special precautions, laboratory setup, experiences, trainings. So, the researchers around the globe have developed different brominating reagents to make bromination procedure hassle-free (Saikia, Borah \& Phukan, 2016).

\section{LITERATURE REVIEW}

In this context, use of various $\mathrm{N}$-bromo compounds and organic ammonium tribromides as brominating agents have gained a special attention (refer to figure 1).

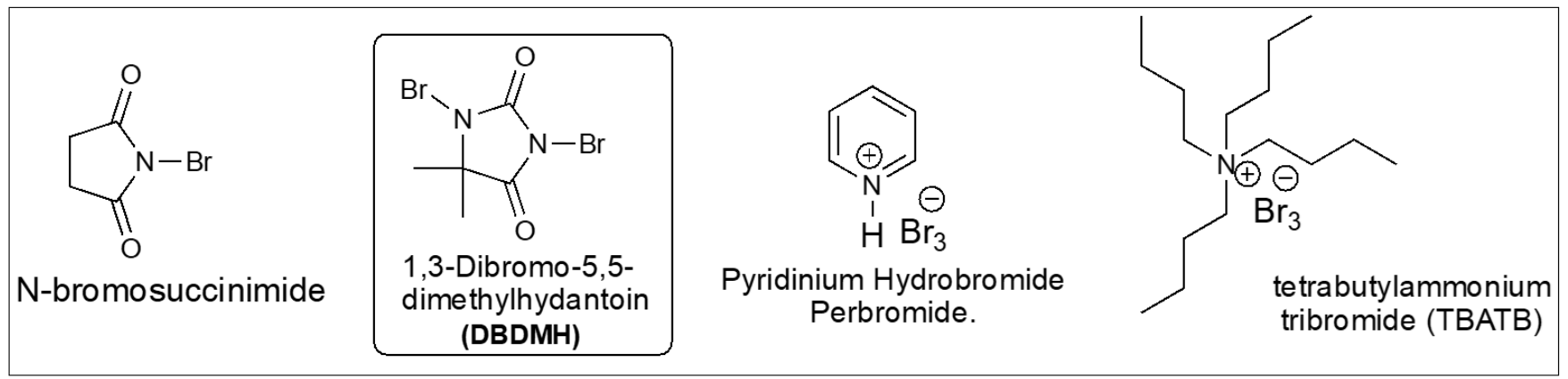

Figure 1: Various brominating agents

1,3-Dibromo-5,5-dimethylhydantoin or DBDMH is a derivative of dimethylhydantoin which contains two bromine atoms. It is commercially available as white powder or crystals. DBDMH is usually prepared in laboratory by reaction of $\mathrm{Br}_{2}(1 \mathrm{~mol})$ with 5,5 -dimethylhydantoin $(0.5 \mathrm{~mol})$ and $\mathrm{NaOH}(1$ $\mathrm{mol}$ ) in ice-water at $0 \circ \mathrm{C}$ (Virgil, 2001). 
It has been widely known as a brominating and oxidizing agent. DBDMH is used as source for bromine in the radical bromination of benzylic positions, allylic positions and various electrophilic additions of alkenes, alkynes, arenes and heterocycles. An analogous brominating agent like DBDMH knows as NBS ( $\mathrm{N}$-bromo succinimide) reacts in similar way in many reactions as DBDMH does and known more before the DBDMH. However, many comparative literature studies between NBS and DBDMH proved that DBDMH works better than NBS. DBDMH has a common advantage over NBS and molecular bromine that DBDMH is very easy to handle in laboratory, whereas molecular bromine is very corrosive in nature, for brominating purposes DBDMH exhales NBS as former is the source of two Bromine atoms and later is only of one.

Many synthetic uses of DBDMH have been reported to date. In this book chapter, the unique brominating ability of DBDMH has been discussed. A special attention has been devoted to bromination of aromatic compounds mediated by DBDMH. The selectivity and practicability issues of bromination methods have been pointed out.

\section{DISCUSSION}

\section{Bromination Reactions using DBDMH}

\section{A. Bromination of unsaturated C-C bonds:}

Development of new synthetic technique for di-bromination of unsaturated C-C bonds is very much demanding transformation because the dibrominated compounds are important intermediates in syntheses of many natural product derivatives, pharmaceuticals.

Hernández-Torres, Tan \& Barbas III (2012) disclosed an organocatalytic stereospecific dibromination of a wide variety of functionalized alkenes using $\mathrm{DBDMH}$. They found out a simple thiourea catalyst which accelerated the rate of the dibromination reaction at room temperature. The isolated yield of the desired dibrominated product were found to be good in various screened solvents, including aqueous solvents (Scheme 1).

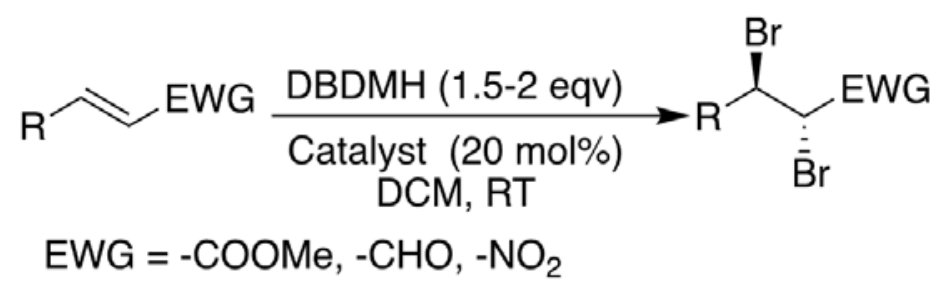<smiles>CCCNC(=S)Nc1cc(C(F)(F)F)cc(C(F)(F)F)c1</smiles>
Thiourea Catalyst

\section{Scheme 1: Di-bromination of alkenes using DBDMH}

$\alpha-M o n o$ and $\alpha, \alpha^{\prime}$-dihalo ketones are very important structural motifs and useful synthons for the fine organic chemicals, agrochemicals, pharmaceuticals and natural products. These types of brominated compound can be synthesised from various alkynes using DBDBMH as the brominating agent. Dr W.M. He et al. has developed an efficient method for the selective synthesis of $\alpha$-mono-bromo or $\alpha, \alpha^{\prime}$-dibromo ketones via a water-controlled three-component thiourea-catalysed cascade reaction (Scheme 2). They have judiciously switched the product formation depending on the reaction solvent (Wu et al. 2017). When the reactions were conducted in aqueous acetone medium, a-monobromo ketones are produced completely selectively. On the other hand, $\alpha, \alpha^{\prime}$-dibromo ketones are exclusively formed when water was used as the sole solvent.

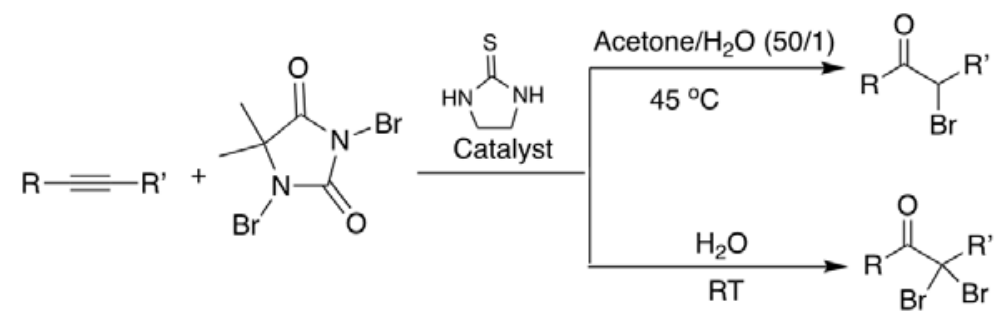

Scheme 2: $\alpha-M o n o$ and $\alpha, \alpha^{\prime}-d i b r o m o$ ketones preparation from alkynes using DBDMH 
a-Bromoacetals of ketones are very useful as intermediates in the synthesis of biologically active compounds specially for triazole fungicides and many more. DBDMH was used as an oxidising as well as brominating agent to prepare $\alpha$-Bromoacetals of ketones from secondary alcohol and ethylene glycol through oxidation, bromination, and acetalization process in one pot methodology (Scheme 3) (Han et al. 2017).

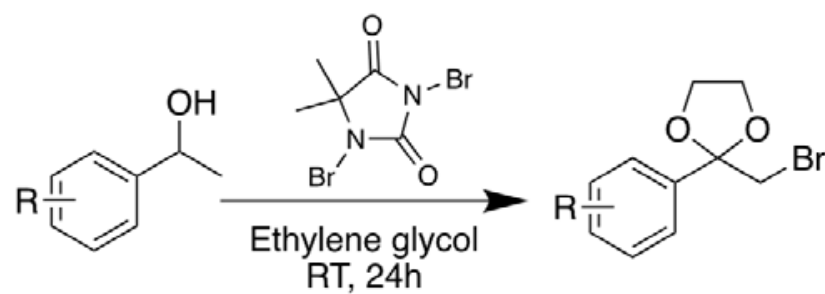

\section{Scheme 3: Synthesis of $\alpha$-Bromoacetals of ketones from secondary alcohol and ethylene glycol mediated by DBDMH}

\section{B. Bromination of Aromatic Compounds:}

Bromination of aromatic compounds (benzylic or ring substitution) are widely practiced reaction in organic synthesis and material science. Specially, recent development of metal-catalyzed coupling reaction increased the utilization of bromine substituted aromatic compound. $\mathrm{N}$-bromo succinimide (NBS) is a frequently used reagent for benzylic as well as aromatic electrophilic substitution, but the drawback of this process is that this requires high temperature or highly activated aromatic compounds.

\section{B-1 Benzylic bromination}

Benzylic bromination happens through a free radical chain reaction. Like the usual course of free radical reaction, benzylic bromination involves three main steps: initiation, propagation, and termination. The bromine radical, $\mathrm{Br}$ which forms from $\mathrm{N}$-bromo compounds through heat or light can break the weaker benzylic C-H bonds for effective bromination at benzylic position.<smiles>CCc1ccc(Br)cc1</smiles>

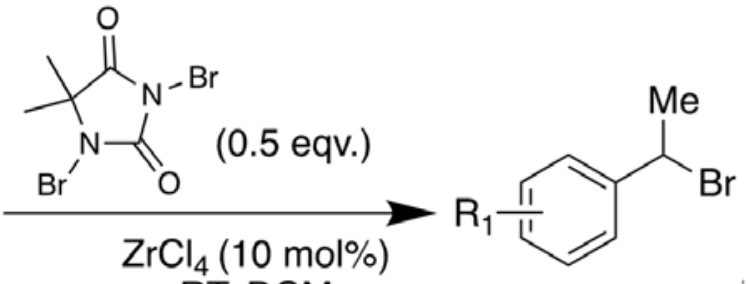

RT, DCM

\section{Scheme 4: Lewis acid catalyzed benzylic bromination of aromatic compounds with DBDMH}

Prof. Hisashi Yamamoto et al disclosed the highly effective Lewis acid catalysed benzylic bromination of aromatic compounds with DBDMH as a brominating reagent (Scheme 4). They found out that the use of zirconium (IV) chloride catalyst (10 mol\%) furnishes the excellent yields of various benzyl bromide derivatives. They were also successful in monobromination of tetramethylsilane with zirconium (IV) chloride and DBDMH (Shibatomi et al. 2008). It may be noted here that in contrast to Lewis acid catalysis which lead to benzylic bromination, Brønsted acid promoted aromatic- ring bromination, devoid of any benzylic bromination.

\section{B-2 Ring Bromination of Aromatic Compounds}

1,3-dibromo-5.5-dimethylhydantoin (DBDMH) is a cheap, easy to handle and above all quite selective bromine less brominating reagent. Therefore, it has attracted a deep attention to test its efficacy for bromination of various aromatic derivatives substituted with either electron donating groups (EDG) or electron withdrawing groups (EWG).

While working with the total synthesis of Lycopodium alkaloid Huperzine A, Dr Yves Langlois and his coworkers developed a synthetic methodology for regioselective bromination of 2-Methoxy-6-methyl pyridine to 5-Bromo-2-methoxy-6-methyl pyridine (Chassaing et al. 1997). They found out that 
DBDMH is the key brominating agent for this successful transformation (Scheme 5).

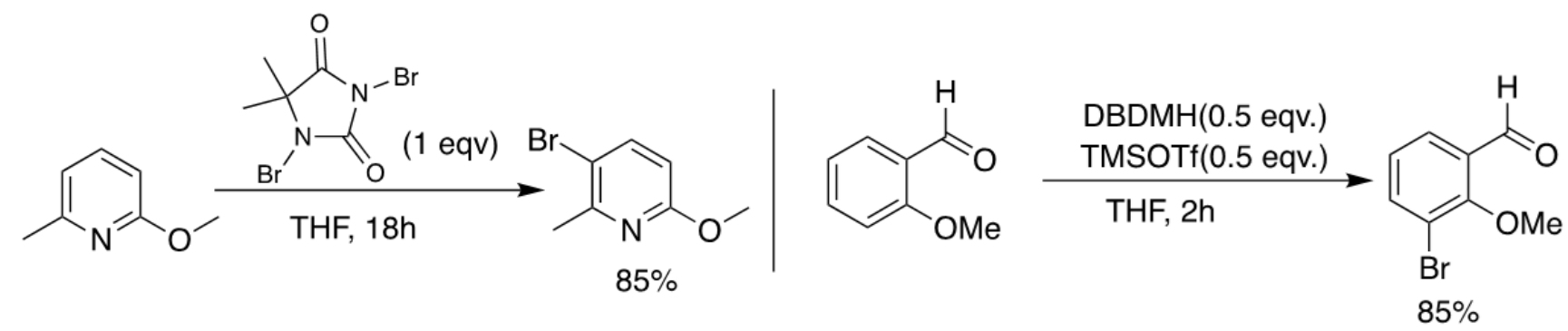

\section{Scheme 5: Regioselective bromination using DBDMH}

At the same time, they established that DBDMH can be activated for the bromination of comparatively electron poor aromatic ring using trimethylsilyl trifluoromethanesulfonate (TMSOTf). 3-Bromo-0anisaldehyde was prepared from o-anisaldehyde using TMSOTf as the promoter (Scheme 5). It may be noted here that without use of TMSOTf no product is formed. The authors have proposed the generation of a bromonium triflate (BrOTf) as reactive intermediate (Scheme-6) in the reaction medium. After electrophilic substitution, one equivalent of triflic acid $(\mathrm{TfOH})$ was released along with silyl enol intermediated. Then, triflic acid reacts with the silyl enol intermediate to produce 5.5dimethylhydantoin (DMH) and a second equivalent of bromonium triflate (Scheme 6).

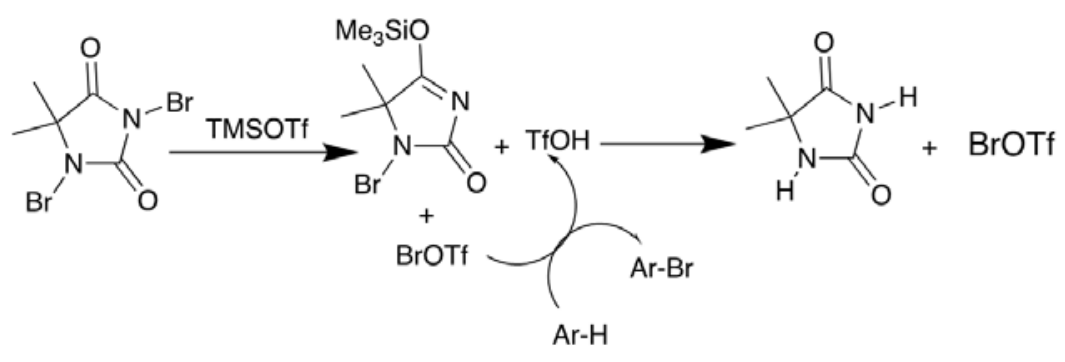

\section{Scheme 6: Activation of DBDMH by TMSOTf}

Regioselective bromination is a challenging task. Bromination of phenolic compounds produce a mixture of ortho/para products. Alam et al. (2005) devised a synthetic methodology for orthomonobromination of phenols and polyphenols using DBDMH as the brominating agent in chloroform solvent medium at room temperature (Scheme 7). They have been successful to synthesize the ortho mono brominated products (X-Z, Scheme 7$)$ using DBDMH in a regioselective manner.

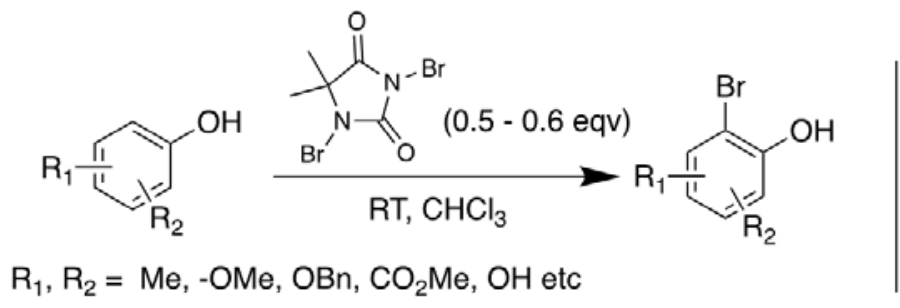

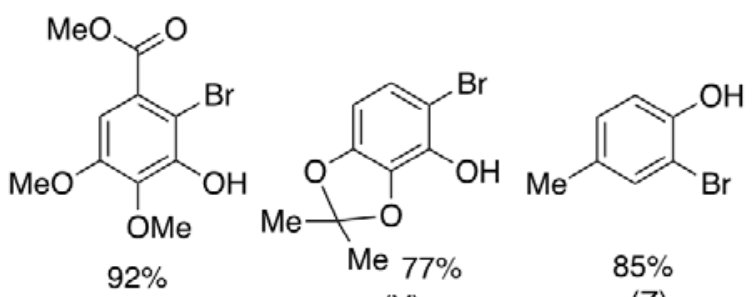

$(\mathrm{X})$

$(\mathrm{Y})$

(Z)

\section{Scheme 7: Regioselective ortho-monobromination of phenols}

Ring bromination of aromatic compounds with electron withdrawing substituents can be carried out using DBDMH as the brominating agent. As a promoter, a strong acid whose pKa values are lower than -2 (such as $\mathrm{CF}_{3} \mathrm{SO}_{3} \mathrm{H}, \mathrm{CH}_{3} \mathrm{SO}_{3} \mathrm{H}, \mathrm{PhSO}_{3} \mathrm{H}, \mathrm{HClO}_{4}, \mathrm{H}_{2} \mathrm{SO}_{4}$ etc.) should be used to achieve a practical yield of the product (Scheme 8) (Fujisaki et al. 1994). The plausible mechanism of ring bromination includes the steps as follows-(a) DBDMH is protonated by strong acids (b) protonated DBDMH generates bromonium ion $(\mathrm{Br}+)$ and $(\mathrm{c})$ finally $\mathrm{Br}+$ is attacked by the aromatic ring (Scheme 8$)$. 


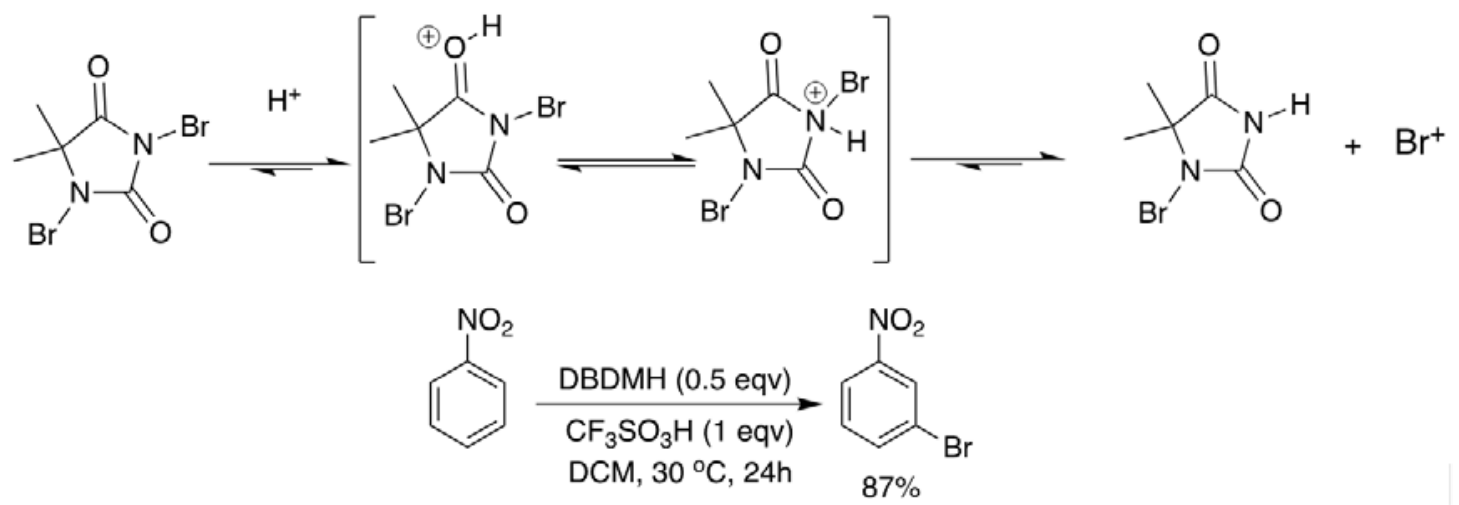

\section{Scheme 8: Ring Bromination of electron poor aromatic compound by DBDMH}

Szumigala et al. (2004) demonstrated an easily scalable, reproduceable synthesis of various aryl bromides through halodeboronation of a series of aryl boronic acids using DBDMH as the brominating reagent and $5 \mathrm{~mol} \% \mathrm{NaOMe}$ (Scheme 9). As a representative example of this methodology, they have reported the synthesis of 2-bromo-3-fluorobenzonitrile from 2-cyano-6-fluorophenylboronic using $\mathrm{DBDMH}$ (Scheme 9).

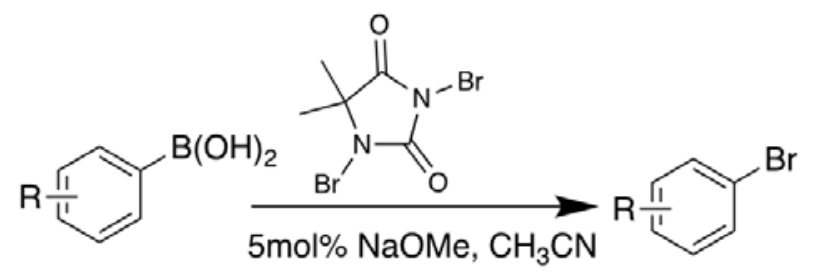<smiles></smiles>

\section{Scheme 9: Halodeboronation by DBDMH}

Most of the aromatic halogenation reactions are usually carried out in chlorinated solvent (e.g., DCM, Chloroform) medium which is toxic in nature. Prof. Yeung research group introduced an environmentally friendly and sustainable pathway for the synthesis of valuable bromoarenes (Shi et al. 2018). They have demonstrated the bromination of aromatic compounds using an indole-based organocatalysts in the non-polar reaction medium, heptane (Scheme 10). The most interesting feature of their development is halogenation of thioarenes which readily undergo oxidative side-reactions in usual bromination conditions.

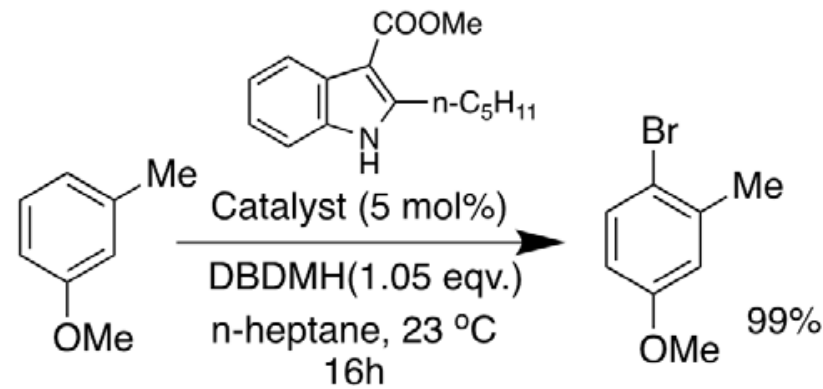

Scheme 10: Indole-based organocatalyst catalyzed aromatic bromination where DBDMH is the brominating agent

Prof. Arai research group have introduced an interesting method of activation of DBDMH for effective electrophilic aromatic bromination using organic disulfide compound (Scheme 11) (lida et al. 2019). Here it is assumed that the disulfide activates the DBDMH as a Lewis base to promote bromination in acetonitrile under mild conditions. 


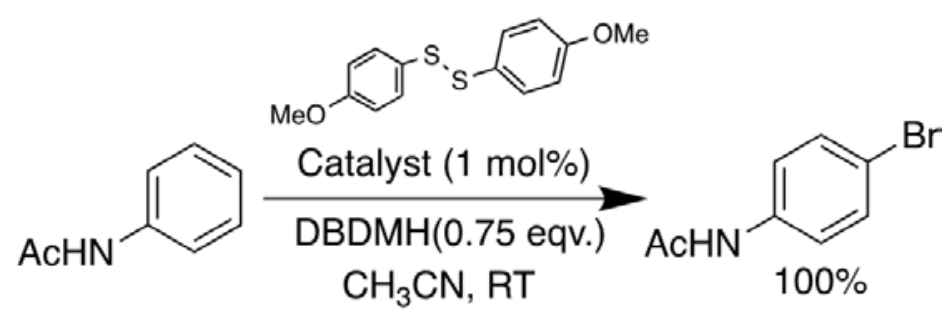

\section{Scheme 11: Disulfide catalysed aromatic bromination where DBDMH is the brominating agent}

DBDMH has been used as a suitable reagent of choice for enantioselective bromination process. Barrett \& Miller (2013) revealed the enantioselective synthesis of atropisomeric benzamides using a simple tetrapeptide bearing a tertiary amine organocatalyst that acts as a Brønsted base for electrophilic bromination (Scheme 12).

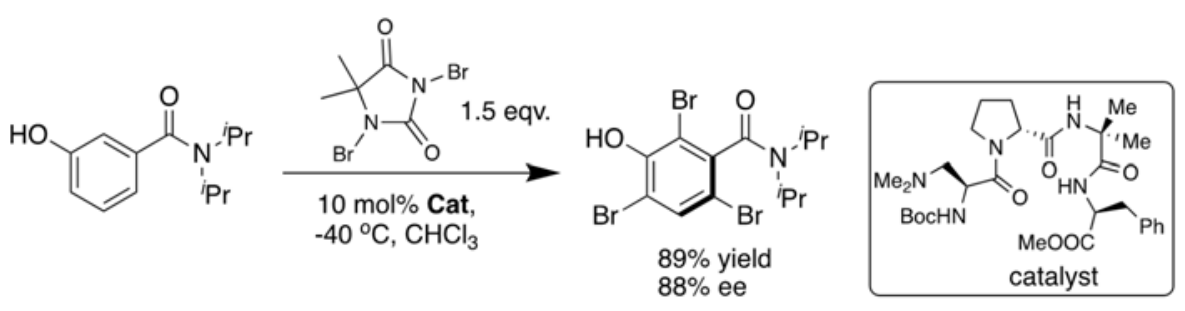

Scheme 12: Use of DBDMH as brominating agent in enantoselective bromination reaction CONCLUSION

In conclusion, DBDMH mediated various organic bromination reaction of synthetic importance has been documented in this book chapter. DBDMH is proved to be very efficient, easy to handle and cheap brominating agent for the bromination of unsaturated $\mathrm{C}-\mathrm{C}$ bond, benzylic position, and aromatic ring. The selectivity issues while bromination using DBDMH have been pointed out. Considering the higher bromine content, stability, and unique reactivity, DBDMH should be considered one of the preferred brominating agents in organic synthesis.

\section{ACKNOWLEDGEMENT}

The authors acknowledge the support of Department of Biotechnology, Government of India for providing DBT-STAR College grant, under which this review project was conducted. The authors also like to thank the Principal and the DBT-STAR Coordinator, Surendranath College, for their support and encouragement in implementing this review project at undergraduate level.

\section{REFERENCE}

Alam, A., Takaguchi, Y., \& Tsuboi, S. (2005). 1, 3-Dibromo-5, 5-dimethylhydantoin, a useful reagent for ortho-monobromination of phenols and polyphenols. 岡山大学環境理工学部研究報告, 10(1), 105109.

Barrett, K. T., \& Miller, S. J. (2013). Enantioselective synthesis of atropisomeric benzamides through peptide-catalyzed bromination. Journal of the American Chemical Society, 135(8), 2963-2966.

Chassaing, C., Haudrechy, A., \& Langlois, Y. (1997). 1, 3-Dibromo-5, 5-dimethylhydantoin, a useful reagent for aromatic bromination. Tetrahedron Letters, 38(25), 4415-4416.

Desmurs, J. R., Goldstein, M. J., \& Gérard, B. (Eds.). (1995). Advances in Organobromine Chemistry II. Elsevier.

Eguchi, H., Kawaguchi, H., Yoshinaga, S., Nishida, A., Nishiguchi, T., \& Fujisaki, S. (1994). Halogenation using N-halogenocompounds. II. Acid catalyzed bromination of aromatic compounds with 1, 3dibromo-5, 5-dimethylhydantoin. Bulletin of the Chemical Society of Japan, 67(7), 1918-1921. 
Han, B., Zheng, Z., Wu, F. \& Wang, A. (2017). One-pot synthesis of a-bromoacetals of ketones from secondary alcohols and 1,3 dibromo-5,5-dimethylhydantoin (DBDMH) in ethylene glycol. Synthetic Communication, 47(24), 2387-2394.

Hernández-Torres, G., Tan, B., \& Barbas III, C. F. (2012). Organocatalysis as a safe practical method for the stereospecific dibromination of unsaturated compounds. Organic Letters, 14(7), 1858-1861.

lida, K., Ishida, S., Watanabe, T., \& Arai, T. (2019). Disulfide-catalyzed iodination of electron-rich aromatic compounds. The Journal of Organic Chemistry, 84(11), 7411-7417.

Saikia, I., Borah, A. J., \& Phukan, P. (2016). Use of bromine and bromo-organic compounds in organic synthesis. Chemical Reviews, 116(12), 6837-7042.

Shi, Y., Ke, Z., \& Yeung, Y. Y. (2018). Environmentally benign indole-catalyzed position-selective halogenation of thioarenes and other aromatics. Green Chemistry, 20(19), 4448-4452.

Shibatomi, K., Zhang, Y., \& Yamamoto, H. (2008). Lewis acid catalyzed benzylic bromination. Chemistry-An Asian Journal, 3(8-9), 1581-1584.

Szumigala, R. H., Devine, P. N., Gauthier, D. R., \& Volante, R. P. (2004). Facile synthesis of 2-bromo-3fluorobenzonitrile: An application and study of the halodeboronation of aryl boronic acids. The Journal of Organic Chemistry, 69(2), 566-569.

Virgil, S. C. (2001). 1, 3-Dibromo-5, 5-dimethylhydantoin. Encyclopedia of Reagents for Organic Synthesis.

Wu, C., Xin, X., Fu, Z. M., Xie, L. Y., Liu, K. J., Wang, Z., ... \& He, W. M. (2017). Water-controlled selective preparation of $\alpha$-mono or $\alpha, \alpha^{\prime}$-dihalo ketones via catalytic cascade reaction of unactivated alkynes with 1, 3-dihalo-5, 5-dimethylhydantoin. Green Chemistry, 19(8), 1983-1989. 


\title{
Synthesis, Characterization and Structure Modelling of Graphene Oxide
}

\author{
Shashi Kant Singh, Hari Shankar Biswas* \\ Department of Chemistry, Surendranath College, Kolkata, India \\ ${ }^{*}$ Corresponding Author's Email: harishankarb7@gmail.com
}

\begin{abstract}
Graphene Oxide (GO), being an important derivative of graphene, has drawn a wide attention from the researchers worldwide. Several innovative methods have been developed today for the synthesis of graphene oxide; however, the large-scale production is highly important for the industrial as well as other commercial applications. One of the most common method for the graphene oxide preparation is the oxidation of graphite to graphene oxide using strong oxidising agents in presence of concentrated acids followed by thermal exfoliation. If graphene oxide thus obtained is reduced, it yields reduced graphene oxide. In this review, the authors have discussed about the synthesis of graphene oxide through different approaches including classical and recent methods. Characterization of the product using FTIR, Raman Spectra, UV-vis, XRD, SEM/TEM imaging, and thermogravimetric analysis (TGA) techniques and structural modelling of $\mathrm{GO}$ is also discussed.
\end{abstract}

Keywords: Graphene Oxide; Synthesis; Methods; Characterization; Structure

\section{INTRODUCTION}

Graphene is a monolayer sheet of sp2-bonded carbon atoms in a hexagonal arrangement, which results into the formation of honeycomb-like 2D lattice (Dresselhaus, Jorio \& Saito, 2010, Geim \& Novoselov, 2010, Sykes, 2009). In the recent years, it has enjoyed great attentions from scientists and engineers due to its excellent properties and huge applications in various fields of research and developments, especially, in electronics and energy storing devices (Eigler \& Hirsch, 2014, Choi \& Lee, 2019). Like graphene, Graphene Oxide is also a monolayer sheet of graphite oxide and contains different oxygen-containing functional group, such as hydroxyl $(-\mathrm{OH})$ and epoxy groups present at the basal plane, while carboxyl $(-\mathrm{COOH})$, carbonyl $(>\mathrm{C}=\mathrm{O})$ and phenol groups are attached to the edges of the plane (Kim, Cote \& Huang, 2010, Eda \& Chhowalla, 2010, Li et al., 2008).

The first ever synthesis of $\mathrm{GO}$ is honoured to Brodie, back to 1859. He used graphite and $\mathrm{KClO}_{3} /$ fuming $\mathrm{HNO}_{3}$ mixture for preparation. In 1898, nearly forty years later, Staudenmaier proposed his method using concentrated $\mathrm{H}_{2} \mathrm{SO}_{4}$, fuming $\mathrm{HNO}_{3}$ and $\mathrm{KClO}_{3}$ as an oxidising mixture. Both methods were hazardous and explosive in nature. In 1958, Hummers and Offeman brought an alternative method for synthesis using $\mathrm{KMnO}_{4}, \mathrm{NaNO}_{3}$ and concentrated $\mathrm{H}_{2} \mathrm{SO}_{4}$. Despite having some flaws, this method was proved to be the most common used method even today. However, Marcano et al. in 2010, proposed an improved form of Hummers method, which was much safer and advantageous. Apart from this, graphene oxide can also be synthesised by using a different carbon source called HDLC (Hydrogenated Diamond like Carbon). Recently, in 2015, Peng introduced a green method for the synthesis using $\mathrm{K}_{2} \mathrm{FeO}_{4}$ as an oxidant.

\section{DISCUSSION}

\section{Synthesis}

Graphene Oxide is generally prepared by the oxidation of graphite into graphite oxide followed by sheet exfoliation either by sonication or by giving some thermal shock. However, the synthesis can also be carried out either through dry or wet approach. The former involves the oxidation of graphene through atomic oxygen under ultrahigh vacuum conditions (Hossain et al., 2012 and Vinogradov et al., 2011), to exposure to molecular oxygen (Starodub, Bartelt \& McCarty, 2010 and Yamamoto et al., 
2012), and treating with ozone under ultraviolet light (Chung et al., 2012 and Huh et al., 2011), while in the latter, graphite is used instead of graphene to make the process cost efficient. In wet synthesis, graphite is oxidised by using strong acids and strong oxidising agents as suggested by Brodie, Staudenmaier, and Hummers.

\section{Methods}

Today, a number of methods are available for synthesis, however, Brodie (Brodie, 1859); Staudenmaier (Staudenmaier, 1898) and Hummers (Hummers \& Offemann, 1958) method are the three major synthetic methods among which Hummers method is the most common one. Based on these methods, several methods are being derived by making major or minor improvements in the methods to get better yield and quality product (refer to table 1).

Table 1: Summary of the Synthetic Methods of Graphene Oxide

\begin{tabular}{|l|c|c|c|}
\hline & Oxidants & Solvents & Additive \\
\hline Staudenmaier & $\mathrm{KClO}_{3}$ & Fuming $\mathrm{HNO}_{3}$ & - \\
\hline Hummers & $\mathrm{KClO}_{3}$ & $\begin{array}{c}\text { Fuming } \mathrm{HNO}_{3} \& \\
\text { conc. } \mathrm{H}_{2} \mathrm{SO}_{4}\end{array}$ & - \\
\hline Tour & $\mathrm{KMnO}_{4}$ & Conc. $\mathrm{H}_{2} \mathrm{SO}_{4}$ & $\mathrm{NaNO}_{3}$ \\
\hline HDLC & $\mathrm{KMnO}_{4}$ & Conc. $\mathrm{H}_{2} \mathrm{SO}_{4}$ & $\mathrm{H}_{3} \mathrm{PO}_{4}$ \\
\hline Peng & $\mathrm{KMnO}_{4}$ & Conc. $\mathrm{H}_{2} \mathrm{SO}_{4}$ & - \\
\hline
\end{tabular}

\section{Brodie Method}

In 1859, a British chemist B.C. Brodie (Brodie, 1859), for the first-time synthesised Graphene Oxide by oxidising the slurry of graphite with a strong oxidising mixture of $\mathrm{KClO}_{3}$ and fuming nitric acid.

Experimental procedure - a mixture of (1:3 weight) graphite and potassium chlorate $\left(\mathrm{KClO}_{3}\right)$ was prepared and the resulting mixture was transferred in a retort. Thereafter, enough fuming nitric acid was added to the above mixture to make a solution. The retort was then kept in a water bath for 3 to 4 days at $60^{\circ} \mathrm{C}$ for carrying out the oxidation process. The completion of reaction was ensured when the yellow vapours stop evolving. Obtained substance was then transferred into plenty of water and washed by decantation to make it free from acids and salts followed by drying in a water bath. Now this whole oxidation procedure was repeated for at least four times to ensure better oxidation of the substance. The product thus obtained was first dried under vacuum and then at $100^{\circ} \mathrm{C}$ to obtain the final product, i.e., graphene oxide.

However, the method was very time consuming and involves health, safety, as well as environmental hazards due to the generation of highly explosive $\mathrm{ClO}_{2}$ gas, toxic $\mathrm{NO}_{x}$ gases that is also a potent air pollutant and carcinogenic $\mathrm{ClO}^{-}$. Moreover, the product yield is also very low.

\section{Staudenmaier Method}

In 1898, L. Staudenmaier (Staudenmaier, 1898) made some modifications in Brodie method and introduced his synthetic method. He kept the oxidising mixture same, i.e., fuming nitric acid and $\mathrm{KClO}_{3}$, but in addition to it, he also added some concentrated Sulphuric Acid to make the reaction medium more acidic (Pendolino \& Armata, 2017). This was done to increase the extent of oxidation. Instead of direct addition of solid $\mathrm{KClO}_{3}$, he added it in several aliquots.

Experimental procedure - At first a mixture of $87.5 \mathrm{~mL}$ concentrated $\mathrm{H}_{2} \mathrm{SO}_{4}(98 \%)$ and $27 \mathrm{~mL}$ fuming 
$\mathrm{HNO}_{3}(98 \%)$ is cooled in an ice bath for 30 mins and to this mixture, $5 \mathrm{~g}$ of graphite is added with vigorous stirring to homogenise the mixture. $55 \mathrm{~g}$ of potassium chlorate was added slow to the reaction mixture kept in the ice bath. Slow addition was chosen to ensure that there should not be any sudden rise in temperature and to avoid the formation of explosive $\mathrm{ClO}_{2}$ gas. Thereafter, the reaction mixture is kept under vigorous stirring continuously for $96 \mathrm{~h}$ at room temperature providing some space for the gas evolved during the reaction to escape. The product obtained after oxidation is washed with $3 \mathrm{~L}$ of deionised water and decanted followed by treatment with $5 \% \mathrm{HCl}$ solution to make it sulphate free and then centrifuged. Finally, the product was washed with deionised water until a negative test for chloride and sulphate is achieved followed by drying in vacuum at $60^{\circ} \mathrm{C}$ for 48 hours to obtain Graphene Oxide.

Despite improvements, Staudenmaier method did not achieve much success. The issue of generation of explosive $\mathrm{ClO}_{2}$ gas and other toxic gases remains the same. The method was still time consuming, and the overall yield was low. Thus, the method still needed some further modifications.

\section{Hummers Method}

Both Brodie's and Staudenmaier's Method faced a failure since the methods were not much efficient, explosive in nature and include severe health impacts. Hence, the methods needed substantial developments. In 1958, Hummers and Offeman (Hummers \& Offeman, 1958) came forward with an alternate method of synthesis with improved safety. They improved the oxidising mixture by replacing $\mathrm{KClO}_{3}$ and fuming $\mathrm{HNO}_{3}$ with $\mathrm{KMnO}_{4}$ and $\mathrm{NaNO}_{3}$. Besides this, the reaction time was also drastically reduced to 2 days that was originally 10 days or even more.

Experimental procedures $-5 \mathrm{~g}$ graphite and $2.5 \mathrm{~g} \mathrm{NaNO}_{3}$ was added to $115 \mathrm{~mL}$ sulphuric acid (98\%) and stirred and then the mixture was cooled in an ice bath. Thereafter, addition of $15 \mathrm{~g} \mathrm{KMnO}_{4}$ was carried out in several portions over a period of 2 hours with vigorous stirring to maintain the temperature below $20^{\circ} \mathrm{C}$. After complete dissolution of $\mathrm{KMnO}_{4}$, the reaction temperature was increased to about $35^{\circ} \mathrm{C}$ and it was maintained for 30 minutes to obtain a brownish-grey paste. After 30 minutes, $250 \mathrm{~mL}$ of water is added to the paste with constant stirring, which results in a violent effervescence and rise in temperature to $98^{\circ} \mathrm{C}$. This temperature was maintained for 15 minutes and after that, the mixture was further diluted with $1 \mathrm{~L}$ of deionised water. After dilution, the mixture was treated with $3 \%$ of $\mathrm{H}_{2} \mathrm{O}_{2}$ solution to eliminate the manganese dioxide formed and unreacted permanganate by converting them into soluble manganese sulphate. The obtained product is then washed with water and decanted. Purification is done via repeated centrifugation and redispersing into deionised water and all the salts and interfering ions are removed. Finally, the product is vacuum dried in oven at $60^{\circ} \mathrm{C}$ for 48 hours to obtain graphene oxide.

Despite being superior to other methods, the extent of oxidation of product obtained in this method was comparable to that of Staudenmaier's one. Moreover, the method did not completely reduce the risk of explosion as the intermediate $\mathrm{Mn}_{2} \mathrm{O}_{7}$ which was formed during the reaction was explosive in nature at elevated temperatures. Furthermore, the GO obtained was found to be contaminated with Mn residues, thus, deteriorating the quality of product. In addition, the emission of toxic gases, such as $\mathrm{NO}_{2}$ and $\mathrm{N}_{2} \mathrm{O}_{4}$, remains as it was. Oxidation time was fast but washing, purifying, and drying steps were time consuming.

\section{Tour Method}

Marcano et al., 2010, made some improvements in Hummers method and proposed the modified form of Hummers method called "Improved Hummers Method." This time the sodium nitrate was replaced with phosphoric acid, and they used a mixture of $\mathrm{H}_{2} \mathrm{SO}_{4} / \mathrm{H}_{3} \mathrm{PO}_{4}(9: 1)$ and $\mathrm{KMnO}_{4}$ in greater amounts as an oxidising agent.

Experimental procedures - At first a mixture of $3 \mathrm{~g}$ graphite and $18 \mathrm{~g} \mathrm{KMnO}_{4}$ was taken and a mixture of concentrated $\mathrm{H}_{2} \mathrm{SO}_{4} / \mathrm{H}_{3} \mathrm{PO}_{4}(360: 40 \mathrm{~mL})$ was added to it which results in slight increase in the temperature to about $35^{\circ} \mathrm{C}$. The mixture was then heated for 12 hours at $50^{\circ} \mathrm{C}$, thereafter, it was cooled to room temperature. The reaction mixture was then poured in $400 \mathrm{~mL}$ of ice and treated with $30 \% \mathrm{H}_{2} \mathrm{O}_{2}$ for 
eliminating any unreacted permanganate residues. The substance obtained was then worked up and filtered through polyester fibre and the filtrate is centrifuged at $4000 \mathrm{rpm}$ for 4 hours, decanting away the supernatant. The substance obtained was washed multiple times with water, $\mathrm{HCl}$ and ethanol. Finally, it was coagulated with $200 \mathrm{~mL}$ ether and filtered over PTFE (Polytetrafluoroethylene) membrane $(0.45 \mu \mathrm{m}$ pore size) and obtained solid was vacuum dried at room temperature overnight to obtain the final product.

On conclusion, this improved method enjoys various advantages over Hummers' method, such as low temperature requirements, no emission of toxic NOx gases and increased safety. The product yield is also quite high and well-oxidised as compared to Hummers' method. Overall, this method can be regarded perfect for the large-scale production of GO.

\section{From HDLC (Hydrogenated Diamond like Carbon)}

Until now, we have talked about preparation of GO using graphite. However, apart from graphite, graphene oxide can also be prepared from many carbon sources and HDLC (Bala et al., 2021) (Hydrogenated Diamond like Carbon) is one of them. HDLC is a very smooth surface material., Before the preparation of GO, HDLC was first converted to A-HDLC (Annealed Hydrogenated Diamond like Carbon). This is done by heating HDLC at high temperature of about $1000^{\circ} \mathrm{C}$ under high vacuum $(\sim$ $10-6$ torr) for 30 minutes and the cooled to room temperature. The synthesis was based on modified Hummers method.

Experimental procedure - A sample of A-HDLC (about $10 \mathrm{~mm}$ diameter) sheet was taken in a reaction jar kept in an ice bath and $1 \mathrm{~mL}$ of ice cooled concentrated sulphuric acid (98\%) was added to it followed by gradual addition of $20 \mathrm{mg} \mathrm{KMnO}_{4}$. While adding $\mathrm{KMnO}_{4}$, the mixture was continuously stirred vigorously with simultaneous cooling of the system so that the reaction temperature remains maintained below $25^{\circ} \mathrm{C}$. After the complete dissolution of $\mathrm{KMnO}_{4}$, the reaction mixture was transferred from ice bath to a water bath where it was heated for half an hour at $35^{\circ} \mathrm{C}$ with continuous stirring and allowing the gas evolved to release. Thereafter, $2 \mathrm{~mL}$ of deionised water was added which results a rapid increase in the temperature to $90^{\circ} \mathrm{C}$ and this temperature was maintained for 10 minutes for better oxidation of the product. The oxidised product thus obtained was purified by filtration, repeated washing, and finally dried under vacuum to the ultimate product, GO.

\section{Peng Method}

In Peng et al., in 2015, introduced a green method for the synthesis of GO by using a strong oxidant, potassium ferrate $\left(\mathrm{K}_{2} \mathrm{FeO}_{4}\right)$. The biggest plus point of this method was the time efficiency of the process. It takes only 1 hour to complete the overall synthesis and thus, it was the faster method compared to others. Moreover, the process was eco-friendly and cheap and is used for large-scale production of single layered GO. Importantly, the sulphuric acid used in the synthesis was recyclable, thus, reducing its consumption.

Experimental procedure - In a reaction flask, $60 \mathrm{~g}$ of $\mathrm{K}_{2} \mathrm{FeO}_{4}, 400 \mathrm{~mL}$ of concentrated sulphuric acid $(93 \%)$ and $10 \mathrm{~g}$ graphite is taken and stirred well and the mixture is kept for 1 hour at room temperature, keeping the flask open to escape the oxygen gas evolved during the reaction. During the reaction, the dark greenish coloured suspension changes to grey coloured viscous fluid. Thereafter, the concentrated sulphuric acid was recycled by centrifuging for 3 minutes at $10000 \mathrm{rpm}$. The as-obtained product was purified by repeated centrifugation and washing out with $1 \mathrm{~L}$ of water until the pH becomes 7 .

\section{Characterization}

GO prepared from the above-mentioned methods was characterized by FT-IR Spectroscopy (Fourier Transform Infrared Spectroscopy), Raman Spectroscopy, UV-Vis (Ultra violet - visible) Spectroscopy, Xray diffraction (XRD), Thermogravimetric Analysis (TGA), TEM and SEM (Scanning Electron Microscopy) techniques. Detailed characterization process and their results are discussed below.

\section{FT-IR Spectroscopy}

FTIR serves as a key technique for characterization since it reveals the information regarding the 
functional groups present in GO (Graphene Oxide). As an illustration, a FTIR spectrum of triple layered $\mathrm{GO}$ at room temperature is shown in figure 1. Hydroxyls shows an absorption at $3000-3700 \mathrm{~cm}-1$ (broad band), carboxyls at 1650-1750 cm-1 and carbonyls at 1750-1850 cm-1. The absorption at 1500-1600 $\mathrm{cm}-1$ corresponds to the $\mathrm{C}=\mathrm{C}$ bond stretching (in-plane), while epoxides appear at $1280-1320 \mathrm{~cm}-1$ and $800-900 \mathrm{~cm}-1$ (Acik et al. 2010).

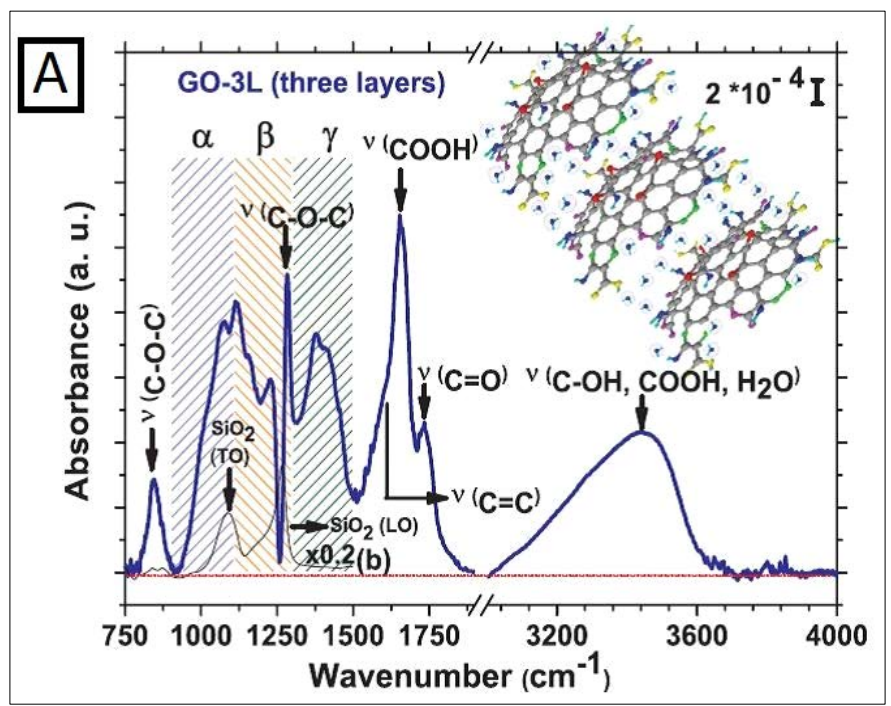

Figure 1: FT-IR spectrum of 3-layered GO printed with permission from Acik et al., 2010

\section{Raman spectroscopy}

Raman Spectroscopy is one of the major tools used for characterization of compounds, especially highly conjugated carbon compounds because the extended double bond conjugations result in highly intense spectrum (Kudin et al., 2008). In the figure 2, the Raman spectra of pristine graphite, GO and reduced GO ( $\mathrm{rGO}$ ) is shown. The spectra reveal a $\mathrm{G}$ band for pristine graphite, while a $\mathrm{G}$ and $\mathrm{D}$ band for both $\mathrm{GO}$ and $\mathrm{rGO}$. The $\mathrm{G}$ band for pristine graphite appears at $1581 \mathrm{~cm}-1$, attributed to the first order scattering of E2g mode (Tuinstra \& Koenig, 1970). For GO, the D band appears at $1363 \mathrm{~cm}^{-1}$, while the $G$ band gets shifted to $1594 \mathrm{~cm}^{-1}$ and is quite broadened compared to that of pristine ones. In contrast, rGO shows its $G$ and $D$ band at 1584 and $1352 \mathrm{~cm}^{-1}$, respectively. It is worth to note the shifting of $G$ and $D$ band of $r G O$ to the lower value in contrast to $G O$, which depicts the decrease in the size of the sp2 framework upon reduction (Tuinstra \& Koenig, 1970). However, the D/G intensity ratio of rGO is found to be greater than GO.

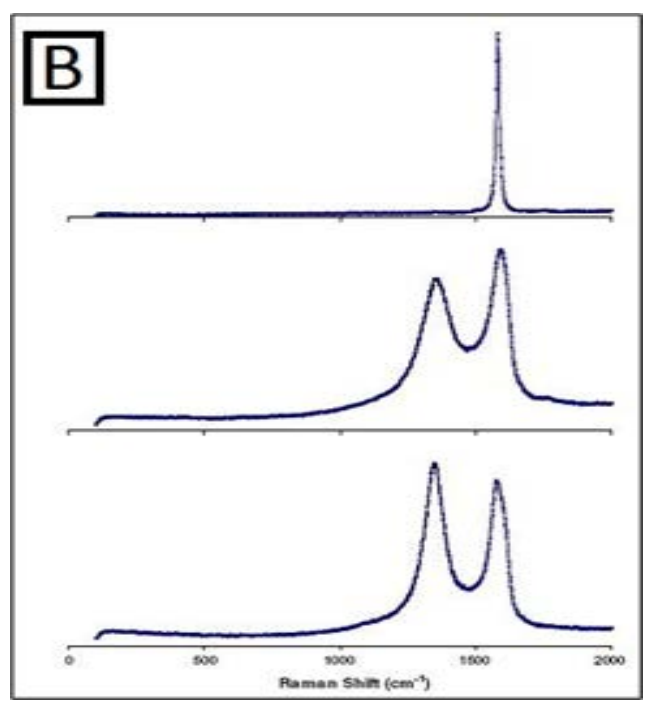

Figure 2: Raman Spectrum of pristine graphite, GO and rGO printed with permission from Stankovich et al., (2007) 


\section{UV-Vis Spectroscopy}

The UV-Vis absorption spectrum of GO exhibits two characteristic absorptions, a maximum at $230 \mathrm{~nm}$ and a shoulder at $300 \mathrm{~nm}$ in figure 3 . The maximum absorption is attributed to the $\pi \rightarrow \pi^{*}$ transition of conjugated $\mathrm{C}=\mathrm{C}$ bonds, whereas the shoulder appears due to $\mathrm{n} \rightarrow \pi^{*}$ transition which corresponds to carbonyl bond (Paredes et al., 2008, and Liu et al., 2015).

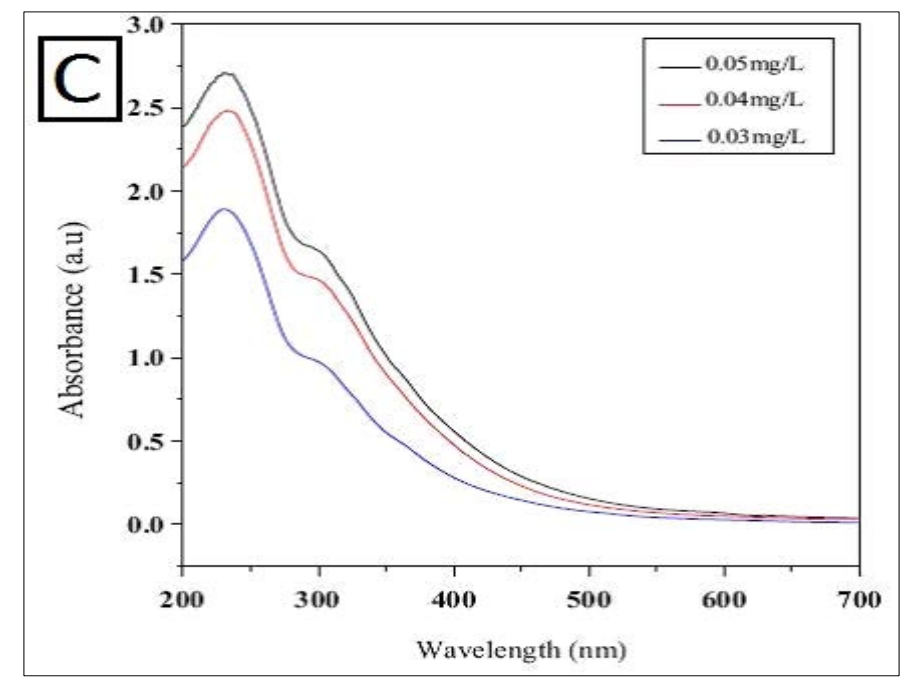

Figure 3: UV-Vis spectra of GO, printed with permission from Liu et al., (2016)

\section{$X$-ray diffraction (XRD)}

X-ray diffraction (XRD) technique is widely used for analysing the structure of crystal lattice. In this technique, a beam of $x$-ray is allowed to fall on the lattice surface which gets scattered after striking the surface by making a scattering angle of $2 \theta$. Thus, by measuring the intensity of this scattered radiation and plotting it against the scattered angle (20), one can obtain the XRD patterns (Compton, 1923). In GO characterization, XRD (X-ray Diffraction) provides the information about the interlayer spacing and hence, it becomes possible to determine the degree of exfoliation of the sheets quantitatively. In the figure 4, the XRD patterns of pristine graphite (a), GO (b) and rGO (c), are shown. From the XRD pattern it can be seen that a very intense peak is obtained for graphite at higher $2 \theta$ value $\left(=26.52^{\circ}\right)$ and the interlayer separation is calculated from the Bragg's Equation and it is found to be $0.34 \mathrm{~nm}$ (Kumar et al., 2012). However, for GO, the intensity of the peak is extremely low and shifted towards the lower $2 \theta$ value which indicates the greater interlayer spacing. Moreover, the value of $2 \theta$ for $\mathrm{GO}$ is largely influenced by the synthetic method used for its preparation and presence of water between the layers (Bragg, 1876). In case of rGO, no such peak is observed which confirms the single-layered structure of rGO.

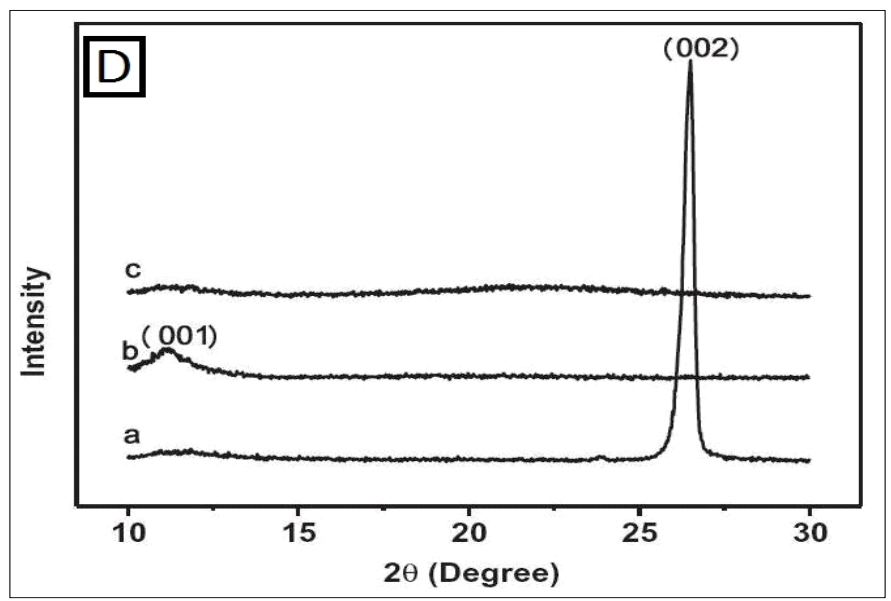

Figure 4: XRD pattern of (a) pristine graphite, (b) GO and (c) rGO, printed with permission from Guo et al. (2012) 


\section{Thermogravimetric Analysis (TGA)}

Thermogravimetric analysis (TGA) is a technique used to monitor the thermal stability of compounds by measuring their mass lost with ascending temperatures. Similarly, the thermal stability of GO can also be determined. From the plot of weight loss vs. temperature of GO figure 5, GO starts losing it weight at $100^{\circ} \mathrm{C}$ and as the temperature reaches to $215^{\circ} \mathrm{C}$ and $550^{\circ} \mathrm{C}$, it shows a drastic drop in weight (Xu et al., 2008). These additional two drops in the weight of $\mathrm{GO}$ are attributed to the loss of $\mathrm{CO}$ and CO2 which ultimately leads to the combustion of GO (Herrera-Alonso et al., 2007, Bissessur et al., 2006, and Wang et al., 2005).

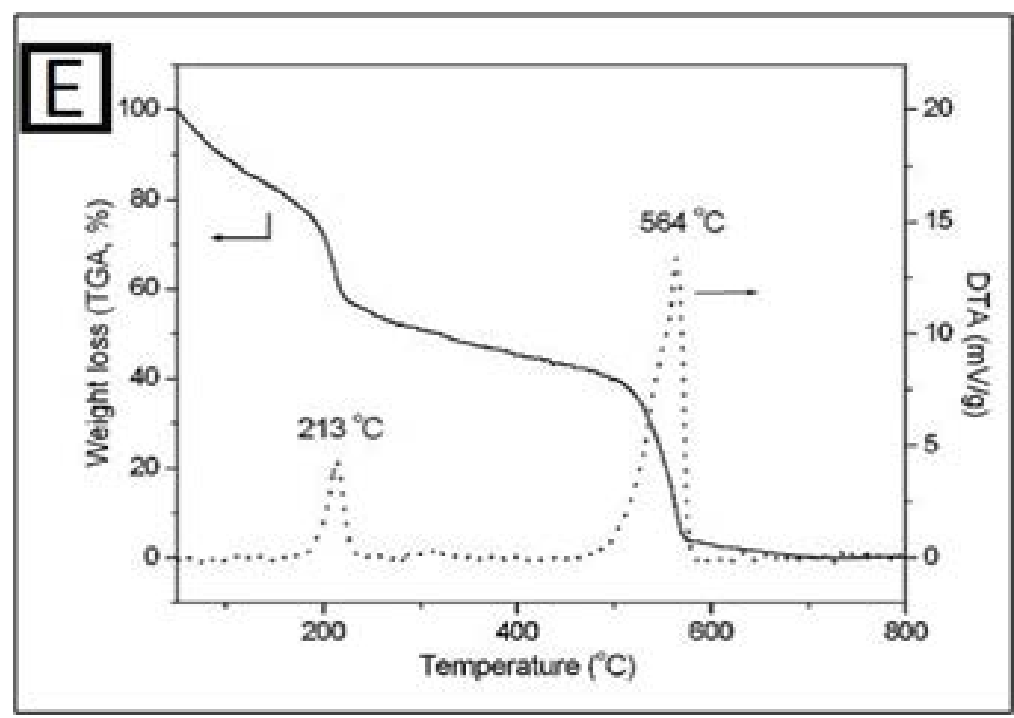

Figure 5: TGA plot of GO, printed with permission from Xu et al., (2008)

\section{Transmission Electron Microscopy (TEM)}

Transmission Electron Microscopy is a microscopy technique widely used in the field of material science and nanotechnology for characterization of samples. In this technique, generally a beam of electrons is allowed to fall on the sample to be characterized. Then the electrons of the incident beam interact with the sample and gets transmitted through it which makes a magnified image of the sample by analysing which, the structural characterization is done. TEM imaging of GO provides information about its layered structure which is found to be helpful in distinguishing between a single layer and multilayer $\mathrm{GO}$. The TEM image of ultrasonically exfoliated GO figure 6 shows a wrinkled and folded sheet of GO (McAllister et al., 2007 and Shao et al., 2010). This wrinkled and folded morphology of the GO proves it to be single layer GO as all of these are its characteristic features (Zhang, Xiong \& Zhao, 2011).

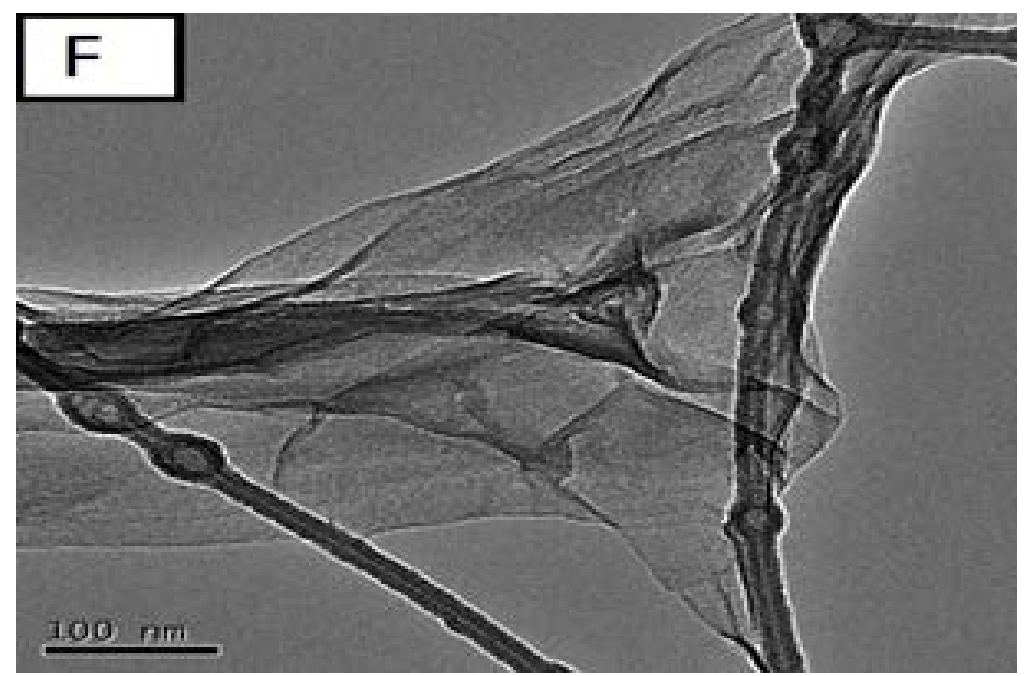

Figure 6: TEM image of GO, printed with permission from Shao et al. (2010) 


\section{Scanning Electron Microscopy (SEM)}

Like TEM, Scanning Electron Microscopy is also an imaging technique used to examine the architectural features of the surface. However, unlike TEM, SEM image are formed by reflected or knocked-off electrons (Shih et al., 1997). The SEM images in the figure 7 showing the morphology of GO having corrugated and wrinkled surface with folded and rough edges (Ren et al., 2013).

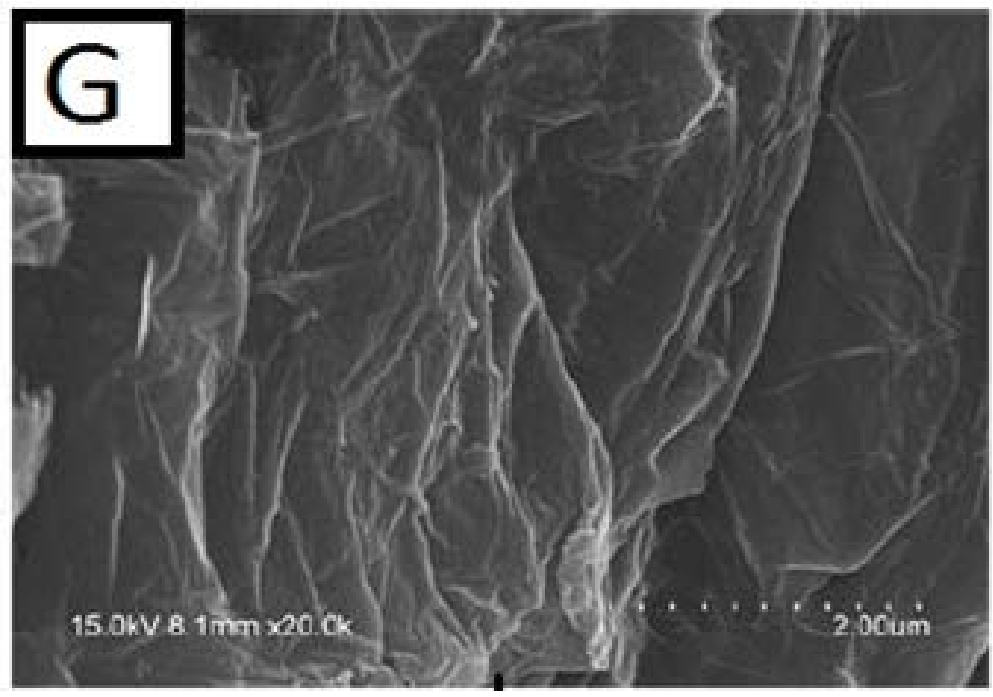

Figure 7: SEM image of GO, printed with permission from Han et al., (2013)

\section{Structure of GO}

The actual structure of GO is not yet clear, and several efforts has been made for its structural prediction. However, some researchers have proposed some approximate models most of which suggests the structure of $\mathrm{GO}$ to be a planar one, having $\mathrm{sp}^{2}$ carbon atoms arranged in hexagonal array and oxygen functional groups being attached to the basal plane. Besides $\mathrm{sp}^{2}$ carbons, there are some $\mathrm{sp}^{3}$ carbons also present on the basal planes of GO having oxygen functionalities. The first ever structure of GO was proposed by Hofmann and Holst (1939) back in the year 1936. Their model was composed of a graphite layer having epoxide functionalities distributed all over the sheet. Later, Ruess (1947) modified Hofmann's model by incorporating hydroxyl functionalities and alternating $\mathrm{sp}^{3}$ carbons into the basal plane. Scholz and Boehm (1969) came forward with their model which involves C=C bonds and periodically cleaved $\mathrm{C}-\mathrm{C}$ bonds within the corrugated carbon layers. The hydroxyl and carbonyl groups are also present but were free from ether oxygen surroundings. Nakajima and Matsuo (1994) introduced their structure of $\mathrm{GO}$ based on the poly $\left(\mathrm{C}_{2} \mathrm{~F}\right) \mathrm{n}$ network thus, forming a stage-2 graphite intercalation compound. Further, Lerf et al. (1998) and Szabó et al. (2006) introduced their model. Both used SSNMR (Solid-State Nuclear Magnetic Resonance) analysis to characterize the structure of GO. Lerf-Klinowski model mainly comprises of tertiary alcohols and 1, 2-ethers, residing on the basal plane of GO. Besides this, the NMR (Nuclear Magnetic Resonance) studies confirmed the presence of $C=C$ bond and ketone groups and suggested that the ketone groups are preferably located at the edges of the GO. However, Lerf et al., (1998) denied the existence of periodically cleaved C —C bond structure. Szabó et al., (2006) suggested a model, which was based on the logic of Scholz-Boehm model and contains 1, 3-ethers attached to the cyclohexane rings.

\section{CONCLUSION}

In this review, the authors have gone through six different methods of synthesis of Graphene Oxide and learned how its synthesis can be made more efficient, safer, faster and environment friendly just by making some alterations in oxidation process, oxidising agent, or source of graphite. Moreover, the results obtained from the instrumental characterization of GO, such as FT-IR, UV-Vis, Raman, XRD, TGA, etc. gives us a clear idea about the presence of various functionalities, sheet exfoliation, thermal stability, etc., while those of SEM and TEM (Transmission Emission Microscopy) images provides a 
close view of the layers which makes our visualization much clearer.

\section{ACKNOWLEDGEMENT}

The authors express their sincere thanks to Department of Biotechnology, Government of India for the funding from DBT-STAR College grant, under which this review project was conducted. The authors are also grateful to the Principal and the DBT-STAR Coordinator, Surendranath College, for their support and encouragement in implementing this review project at undergraduate level.

\section{REFERENCES}

Acik, M., Mattevi, C., Gong, C., Lee, G., Cho, K., Chhowalla, M., \& Chabal, Y. J. (2010). The role of intercalated water in multilayered graphene oxide. ACS Nano, 4(10), 5861-5868.

Bala, S., Bose, R., Chaterjee, S., Sarkar, S., Saha, I., \& Biswas, H. S. (2021). Synthesis of Graphene Oxide from Hydrogenated Diamond Like Carbon and Protein Immobilization onto It: Characterization and Study of Practical Utility. Journal of Materials Science and Chemical Engineering, 9(01), 32.

Bissessur, R., Liu, P. K., White, W., \& Scully, S. F. (2006). Encapsulation of polyanilines into graphite oxide. Langmuir, 22(4), 1729-1734.

Bragg, W. L. (1876). The Crystalline State: The Determination of Crystal Structures, by H. Lipson and W. Cochran (Vol. 3). Cornell University Press.

Brodie, B. C. (1859). XIII. On the atomic weight of graphite. Philosophical Transactions of the Royal Society of London, (149), 249-259.

Choi, W., \& Lee, J. W. (Eds.). (2019). Graphene: Synthesis and Applications. CRC press.

Chung, M. G., Lee, H. M., Kim, T., Choi, J. H., kyun Seo, D., Yoo, J. B., ... \& Kim, Y. H. (2012). Highly sensitive NO2 gas sensor based on ozone treated graphene. Sensors and Actuators B: Chemical, 166, 172-176.

Compton, A. H. (1923). The spectrum of scattered X-rays. Physical Review, 22(5), 409.

Dresselhaus, M. S., Jorio, A., \& Saito, R. (2010). Characterizing graphene, graphite, and carbon nanotubes by Raman spectroscopy. Annu. Rev. Condens. MatterPhys., 1(1), 89-108.

Eda, G., \& Chhowalla, M. (2010). Chemically derived graphene oxide: towards large-area thin-film electronics and optoelectronics. Advanced Materials, 22(22), 2392-2415.

Eigler, S., \& Hirsch, A. (2014). Chemistry with graphene and graphene oxide-challenges for synthetic chemists. Angewandte Chemie International Edition, 53(30), 7720-7738.

Geim, A. K., \& Novoselov, K. S. (2010). The rise of graphene. In Nanoscience and Technology: A Collection of Reviews from Nature Journals (pp. 11-19).

Guo, Y., Sun, X., Liu, Y., Wang, W., Qiu, H., \& Gao, J. (2012). One pot preparation of reduced graphene oxide (RGO) or $\mathrm{Au}(\mathrm{Ag})$ nanoparticle-RGO hybrids using chitosan as a reducing and stabilizing agent and their use in methanol electrooxidation. Carbon, 50(7), 2513-2523.

Han, Y., Wu, Y., Shen, M., Huang, X., Zhu, J., \& Zhang, X. (2013). Preparation and properties of polystyrene nanocomposites with graphite oxide and graphene as flame retardants. Journal of Materials Science, 48(12), 4214-4222.

Herrera-Alonso, M., Abdala, A. A., McAllister, M. J., Aksay, I. A., \& Prud'homme, R. K. (2007). Intercalation and stitching of graphite oxide with diaminoalkanes. Langmuir, 23(21), 10644-10649.

Hofmann, U., \& Holst, R. (1939). Über die Säurenatur und die Methylierung von Graphitoxyd. Berichte der Deutschen Chemischen Gesellschaft (A and B Series), 72(4), 754-771.

Hossain, M. Z., Johns, J. E., Bevan, K. H., Karmel, H. J., Liang, Y. T., Yoshimoto, S., ... \& Hersam, M. C. 
(2012). Chemically homogeneous and thermally reversible oxidation of epitaxial graphene. Nature Chemistry, 4(4), 305-309.

Huh, S., Park, J., Kim, Y. S., Kim, K. S., Hong, B. H., \& Nam, J. M. (2011). UV/ozone-oxidized large-scale graphene platform with large chemical enhancement in surface-enhanced Raman scattering. ACS Nano, 5(12), 9799-9806.

Hummers Jr, W. S., \& Offeman, R. E. (1958). Preparation of graphitic oxide. Journal of the American Chemical Society, 80(6), 1339-1339.

Kim, F., Cote, L. J., \& Huang, J. (2010). Graphene oxide: surface activity and two-dimensional assembly. Advanced Materials, 22(17), 1954-1958.

Kudin, K. N., Ozbas, B., Schniepp, H. C., Prud'Homme, R. K., Aksay, I. A., \& Car, R. (2008). Raman spectra of graphite oxide and functionalized graphene sheets. Nano Letters, 8(1), 36-41.

Kumar, N. A., Choi, H. J., Shin, Y. R., Chang, D. W., Dai, L., \& Baek, J. B. (2012). Polyaniline-grafted reduced graphene oxide for efficient electrochemical supercapacitors. ACS Nano, 6(2), 1715-1723.

Lerf, A., He, H., Forster, M., \& Klinowski, J. (1998). Structure of graphite oxide revisited. The Journal of Physical Chemistry B, 102(23), 4477-4482.

Li, X., Zhang, G., Bai, X., Sun, X., Wang, X., Wang, E., \& Dai, H. (2008). Highly conducting graphene sheets and Langmuir-Blodgett films. Nature Nanotechnology, 3(9), 538-542.

Liu, J., Li, X., Jia, W., Li, Z., Zhao, Y., \& Ren, S. (2015). Demulsification of crude oil-in-water emulsions driven by graphene oxide nanosheets. Energy \& Fuels, 29(7), 4644-4653.

Liu, Y., Zhang, Y., Duan, L., Zhang, W., Su, M., Sun, Z., \& He, P. (2016). Polystyrene/graphene oxide nanocomposites synthesized via Pickering polymerization. Progress in Organic Coatings, 99, 23-31.

Marcano, D. C., Kosynkin, D. V., Berlin, J. M., Sinitskii, A., Sun, Z., Slesarev, A., ... \& Tour, J. M. (2010). Improved synthesis of graphene oxide. ACS Nano, 4(8), 4806-4814.

McAllister, M. J., Li, J. L., Adamson, D. H., Schniepp, H. C., Abdala, A. A., Liu, J., ... \& Aksay, I. A. (2007). Single sheet functionalized graphene by oxidation and thermal expansion of graphite. Chemistry of Materials, 19(18), 4396-4404.

Nakajima, T., \& Matsuo, Y. (1994). Formation process and structure of graphite oxide. Carbon, 32(3), 469-475.

Paredes, J. I., Villar-Rodil, S., Martínez-Alonso, A., \& Tascon, J. M. D. (2008). Graphene oxide dispersions in organic solvents. Langmuir, 24(19), 10560-10564.

Pendolino, F., \& Armata, N. (2017). Synthesis, characterization, and models of graphene oxide. In Graphene Oxide in Environmental Remediation Process (pp. 5-21). Springer, Cham.

Peng, L., Xu, Z., Liu, Z., Wei, Y., Sun, H., Li, Z., ... \& Gao, C. (2015). An iron-based green approach to 1-h production of single-layer graphene oxide. Nature Communications, 6(1), 1-9.

Ren, X., Li, J., Tan, X., \& Wang, X. (2013). Comparative study of graphene oxide, activated carbon and carbon nanotubes as adsorbents for copper decontamination. Dalton Transactions, 42(15), 52665274.

Ruess, G. (1947). Über das graphitoxyhydroxyd (graphitoxyd). Monatshefte für Chemie und Verwandte Teile Anderer Wissenschaften, 76(3), 381-417.

Scholz, W., \& Boehm, H. P. (1969). Untersuchungen am graphitoxid. VI. Betrachtungen zur struktur des graphitoxids. Zeitschrift für Anorganische und Allgemeine Chemie, 369(3-6), 327-340.

Shao, Y., Wang, J., Engelhard, M., Wang, C., \& Lin, Y. (2010). Facile and controllable electrochemical reduction of graphene oxide and its applications. Journal of Materials Chemistry, 20(4), 743-748.

Shih, A., Yater, J., Hor, C., \& Abrams, R. (1997). Secondary electron emission studies. Applied Surface 
Science, 111, 251-258.

Stankovich, S., Dikin, D. A., Piner, R. D., Kohlhaas, K. A., Kleinhammes, A., Jia, Y., ... \& Ruoff, R. S. (2007). Synthesis of graphene-based nanosheets via chemical reduction of exfoliated graphite oxide. Carbon, 45(7), 1558-1565.

Starodub, E., Bartelt, N. C., \& McCarty, K. F. (2010). Oxidation of graphene on metals. The Journal of Physical Chemistry C, 114(11), 5134-5140.

Staudenmaier, L. (1898). Verfahren zur darstellung der graphitsäure. Berichte der deutschen chemischen Gesellschaft, 31(2), 1481-1487.

Sykes, E. C. H. (2009). Surface assembly: graphene goes undercover. Nature chemistry, 1(3), 175.

Szabó, T., Berkesi, O., Forgó, P., Josepovits, K., Sanakis, Y., Petridis, D., \& Dékány, I. (2006). Evolution of surface functional groups in a series of progressively oxidized graphite oxides. Chemistry of Materials, 18(11), 2740-2749.

Tuinstra, F., \& Koenig, J. L. (1970). Raman spectrum of graphite. The Journal of Chemical Physics, 53(3), 1126-1130.

Vinogradov, N. A., Schulte, K., Ng, M. L., Mikkelsen, A., Lundgren, E., Martensson, N., \& Preobrajenski, A. B. (2011). Impact of atomic oxygen on the structure of graphene formed on $\operatorname{Ir}(111)$ and $\mathrm{Pt}$ (111). The Journal of Physical Chemistry C, 115(19), 9568-9577.

Wang, G., Yang, Z., Li, X., \& Li, C. (2005). Synthesis of poly (aniline-co-o-anisidine)-intercalated graphite oxide composite by delamination/reassembling method. Carbon, 43(12), 2564-2570.

Xu, C., Wang, X., Zhu, J., Yang, X., \& Lu, L. (2008). Deposition of Co 304 nanoparticles onto exfoliated graphite oxide sheets. Journal of Materials Chemistry, 18(46), 5625-5629.

Yamamoto, M., Einstein, T. L., Fuhrer, M. S., \& Cullen, W. G. (2012). Charge inhomogeneity determines oxidative reactivity of graphene on substrates. ACS Nano, 6(9), 8335-8341.

Zhang, J., Xiong, Z., \& Zhao, X. S. (2011). Graphene-metal-oxide composites for the degradation of dyes under visible light irradiation. Journal of Materials Chemistry, 21(11), 3634-3640. 


\title{
A Brief Idea About Protein-Protein Interactions Modulators
}

\author{
Saumik Mal, Boijoyanta Howlader, Apurba Biswas* \\ Department of Chemistry, Surendranath College, Kolkata, India
}

${ }^{*}$ Corresponding Author's Email: apurbacu@yahoo.co.in

\begin{abstract}
Protein-protein Interaction (PPIs) play an important role in the life cycle. Many studies have suggested that aberrant PPIs relate to several fatal diseases including cancer, infectious diseases and also neurodegenerative diseases, regulating diseases. Consequently, PPIs, usually small molecule inhibitors, are an important diagnostic strategy for disease progression as well as a crucial technique for medication development. In the past few decades, targeting intracellular PPIs for framed a advances drug is really a challenging task. However, advance in PPIs focused drug discoverytechnology, have been reported and a few have entered clinical trials, with some of them being approved for commercialization, indicating that modulators that target PPIs have a significant advantage.
\end{abstract}

\section{Key words: Protein-Protein Interactions; Small Molecules; Modulators}

\section{INTRODUCTION}

The goal of advances drug discovery is to find new therapeutic substances which has an ability to target disease-specific biological mechanisms or pathways (Díaz-Eufracio, Naveja \& Medina-Franco, 2018). It is considered that the elementary building block of life is the protein which are formed by amino acid. By Gene coding method (translation, central dogma) the amino acids are linked together and construct the peptide bond between them, where from the various proteins are manufactured by the peptides. Then peptides form the living tissues. Besides this proteins have the widest array of biological processes such as catalysts reactions, transport molecules, immune reactions to various pathogens and cell functions by signal transduction methods and also others important biological processes which are directly associated with our health like DNA-replication transcription, translation and also transmembrane signals transductions all depends on specific protein (Voet \& Voet, 2010; Basu et al., 2019; Tewari, 2019). The aforementioned biological activist are controls through specified proteins complex which are typically regulated through protein-protein interactions. PPIs in cell construct a complicated network which has a term named "interactome" which plays a crucial role in physiological and pathological processes like cell proliferation, growth, differentiations and apoptosis etc (Venkatesan et al., 2008; Koh et al.,2012). So many human hazardous diseases are associated with aberrant PPIs such as cancers, infectious disease and neurodegenerative diseases. Protein protein interactions (PPIs) are an appealing new class of molecular targets in this context, and they play a vital role in the course of many disease states (Robertson \& Spring, 2018; Zhang, Andersen \& Gerona-Navarro, 2018).

\section{LITERATURE REVIEW AND DISCUSSION}

\section{Biophysical Principles Controlling the PPIs:}

Guldberg and Waage (1986) in their study of chemical affinity formulated the law of rate of chemical reactions as proportional to the "active mass" of the reagents (Bera, 2019; Schreiber, 2021). This is called the law of mass action and very clearly defined "Active mass" as amount per unit volume. The law of mass action generally helps to understand the dynamic of a chemical reaction. It can be also expressed the concentration of the products divided by those of the reactants is constant for a given temperature and pressure. For a reaction. $A+B \leftrightharpoons A B$, the equilibrium constant $K$ is $[A][B] /[A B]$. From the law of mass action is, it is predicted that the amount of protein in complex is a function of its concentration and the binding affinity between the two proteins. In complex cellular atmosphere, this 
such of situation is more mystical. There are many competing interactions present which interact to bind the protein even weakly. Let us take example that a potential interaction $[A][B]$ is competed with by a maker interaction of $[A]$ with $[C]$, but with $[C]$ being at a 1000 - fold higher concentration than $[B]$. In this case the interaction between $[A]$ with $[B]$ will not be occurred the biological context. The situation is furthermore completed by part-translational modification (glycosylation, phosphorylation, etc) and cofactors (such as metals, peptides, etc) which often transfers a specific protein from binding to nonbindings. The biological system maintains all of the above process to dictate binding and function. From the biological paint of view the ability of the cell and organism to simulate the formation of PPIs is one of the main techniques used to control almost any biological processes, without which life would not possible.

\section{Entropy-Enthalpy Compensation in PPIs:}

Multiple processes govern the association of a protein complex in solution. Some processes are structural rearrangement, desolvation and also formation of new short-range interactions (Schreiber, 2021). When enthalpically favoured complex is formed between two molecules, for this complexation their conformational, rotational and translational freedom are reduced. This is due to the fact that, multiple processes are inter-links with each other, even formation of new 'H'-bonds or ionic interactions may be energetically neutral, due to entropic lass resulting from less of freedom of motion. It has been proposed that enthalpically favourable rearrangements of water were stronger than entropically favourable rearrangement of water. The problem in analysing enthalpy-entropy compensation is that water and molecular motions represent common sources of enormous and potentially compensating differences in enthalpy and entropy between similar binding processes. Such differences are incompatible with common approaches to molecular docking, which rely on strict conformational constraints and implicit water (Nero et al., 2014; Scott et al., 2016). Moreover, binding affinity provides only a partial description of the recognition processes. This has clear implications for force-field development to generate novel protein-interaction partners. Still, most current docking and design algorithms don't consider entropic contributions, due to the difficulties in their implementation.

\section{Challenges in Developing PPIs Modulator:}

Actually, the classic small molecules drug discovery deals with the protein-protein interactions such as enzymes, ion-channel, receptor where a highly specific ligand-binding site is located. It is convenient for the interaction of small molecules. The PPIs modulations through small molecules is actually regarded tough and PPIs were considered as undruggable targets (Stumpf et al., 2008; Buchwald, 2010).

\section{Reason's}

1. First, PPIs take place in the interacting region of the two-specific protein. Experimental results show that the interface site of the interaction is larger than the contact area of the receptor-ligand (Stelzl et al., 2005).

2. Second, the interface of the PPIs avails to be broad and carried some of pockets like shapes which make it inconvenient for the framed of small molecule to bind.

3. Third, if we just observed the traditional drug target like enzymes, ion-channel, there is a deficiency in PPIs for endogenous small molecular ligands as their reference (Lu et al., 2020).

\section{HOT-spots:}

Theoretically the large binding interface are not suitable for the best drug targets as it is quitting ponderous to recognize a similar molecule. Generally, PPIs take place on various amino acid residues on the PPIs interface. The region of the amino acid residue on the PPIs interface that contribute to the binding-free energy are called "hot-spot" (Lu et al., 2020). When the area PPIs prolong, the number of hot-spot is gradually increase. Generally, tryptophan, arginine and tyrosine are arising in hot-spot than others amino acids. As the amino acids and hot-spot play import role for this PPIs for drug design, so 
they are frequently used for purpose solved ((Lu et al., 2020; Coyne, 2010).

\section{Modern Thinking About the Development of PPIs Modulator:}

As the PPIs interface is specified and unique so it is very challenging tasks to target the PPIs (Lu et al., 2020). If we compare to the binding site of the traditional protein targets, interface of the PPIs tends to be broad. Therefore, it is difficult for the classical medicinal chemistry to identify and design the PPIs modulator. So, it is going to be more essential to create more effective and sophisticated approaches for screening the PPIs modulators.

\section{Working Function of PPIs Modulators:}

Many reports suggested that the small molecules PPIs moderators have a high potential to interact with the protein-protein interface and also allosteric sites (Lu et al., 2020). It is also notice there are some modulators which generally help to maintain the inert potential energy of the proteins and also accelerate the binding force between the protein neighbor as an outcome a balanced state is present on there. When the modulators come close to a noninteracting site and get attached to this specific site, a change in conformation of the protein is observed. As an effect of such change the potential of binding of the protein get enriched. On the other hand, when the modulators come towards the PPIs interface, the binding capacity get enhance between to adjacent proteins as the contact area become increase (Lu et al., 2020).

\section{Types of PPIs Modulators:}

\section{Small molecule modulators}

In the earlier time, when a new drug development idea was framed, the scientists are mainly focused on the PPIs interface of the enzymes and ions channels as the interface is broad and quite heave. Later it is observed, the previous method is not fulfilled the actual purpose of drug development; one of the reasons is the deficiency of binding pockets where a small molecule can easily attach. Having poor solubility and large weight, this type of modulator may face pharmacokinetic problem (Wells \& McClendon, 2007). Whereas the small molecule modulator would be a suitable choice for its tight and narrow PPI interface.

\section{Antibody modulator}

It covers the large interface at the targeting process of large PPI interface (Lu et al., 2020). As the monoclonal antibodies have large molecule weight and limited application in extracellular targets, so it completes with PPIs. Now a days the monoclonal antibodies have been played a crucial role in many clinical treatments.

\section{Peptides}

Analyzing the structural information of the hot-spot, the well-organized peptides are designed. The newly designed peptides attached to the proteins and accelerate the proneness with the proteins (Lu et al., 2020). The molecular weight of peptide is quite lower than the small molecule modulators but higher than the monoclonal antibodies. Being high target specific in nature the peptides is an appropriate PPI modulator.

\section{Inhibitor of MDM2/p53Interaction (Small Molecule Modulator and Peptides):}

About: One of the essential proteins is the p53 which generally governs the Primates's cell cycle and arrest the tumor formation. Many reports claimed that modification of the p53 gene is the reason for the half of the human cancer. One kind of protooncogene is the mouse double minute 2 (MDM2). It generally give a negative signal for the $\mathrm{p} 53$ gene. MDM2 restricts the transactivation of $\mathrm{p} 53$ by forming a complex with it. So, breaking the interaction between MDM2 -p53, the impaired function of p53 get recovered.

\section{Structural \& Suppression Strategy:}

The X-ray crystallography revealed the entire information about the interaction mechanism of MDM2 
/p53. From the crystallographic results, it is observed that the four hydrophobic residues (Phel 9 , Leu22, Trp23, Leu26) are involved in the interaction site of MDM2/p53. If a small molecule is designed, will be a feasible technique for the suppression of their functions. The small molecule has an ability to mimics the specific structure oh p53 for a better attachment. As a result of it the inactivation of p53 is restricted.

\section{CONCLUSION}

PPIs modulators development has been possessed a notable place in preclinical studies. The frame of modulators for the purpose of target PPIs is encountered many colossal problems. The deficiency of the suitable ligands, the inconvenient PPI interface, lacks proper guidance methods are the problem for the design an appropriate modulator to target the PPIs. The deficiency of high-resolution PPI protein skeletal information is one of the main barriers for this purpose. However, the fast grow up of structural biology field and corresponding strategies disclosed many unknown sites where a potential progress is hidden about the development of PPIs modulator. It is hoped that more PPI small molecule modulators will be designed and enter the clinic to benefit the patients.

\section{ACKNOWLEDGEMENT}

The authors express their sincere thanks to Department of Biotechnology, Government of India for the funding from DBT-STAR College grant, under which this review project was conducted. They are also grateful to the Principal and the DBT-STAR Coordinator, Surendranath College, for their support and encouragement in implementing this review project at undergraduate level.

\section{REFERENCES}

Arkin, M. R., \& Wells, J. A. (2004). Small-molecule inhibitors of protein-protein interactions: progressing towards the dream. Nature Reviews Drug Discovery, 3(4), 301-317.

Basu, K., Saha, C., Chakraborty, B., \& Chakraborty S. (2019). Analytical Clinical Biochemistry. Techno World.

Bera, N. K. (2019). Physical Chemistry Concept and Models (Vol II). Techno World.

Buchwald, P. (2010). Small molecule protein-protein interaction inhibitors: Therapeutic potential in light of molecular size, chemical space, and ligand binding efficiency considerations. IUBMB Life, 62(10), 724-731.

Coyne, A. G., Scott, D. E., \& Abell, C. (2010). Drugging challenging targets using fragment-based approaches. Current Opinion in Chemical Biology, 14(3), 299-307.

Díaz-Eufracio, B. I., Naveja, J. J., \& Medina-Franco, J. L. (2018). Protein-protein interaction modulators for epigenetic therapies. Advances in Protein Chemistry and Structural Biology, 110, 65-84.

Koh, G. C., Porras, P., Aranda, B., Hermjakob, H., \& Orchard, S. E. (2012). Analyzing protein-protein interaction networks. Journal of Proteome Research, 11(4), 2014-2031.

Lu, H., Zhou, Q., He, J., Jiang, Z., Peng, C., Tong, R., \& Shi, J. (2020). Recent advances in the development of protein-protein interactions modulators: mechanisms and clinical trials. Signal Transduction and Targeted Therapy, 5(1), 1-23.

Nero, T. L., Morton, C. J., Holien, J. K., Wielens, J., \& Parker, M. W. (2014). Oncogenic protein interfaces: small molecules, big challenges. Nature Reviews Cancer, 14(4), 248-262.

Robertson, N. S., \& Spring, D. R. (2018). Using peptidomimetics and constrained peptides as valuable tools for inhibiting protein-protein interactions. Molecules, 23(4), 959.

Schreiber, G. (2020). Protein-Protein Interaction Interfaces and their Functional Implications.

Scott, D. E., Bayly, A. R., Abell, C., \& Skidmore, J. (2016). Small molecules, big targets: drug discovery faces the protein-protein interaction challenge. Nature Reviews Drug Discovery, 15(8), 533-550. 
Stelzl, U., Worm, U., Lalowski, M., Haenig, C., Brembeck, F. H., Goehler, H., ... \& Wanker, E. E. (2005). A human protein-protein interaction network: a resource for annotating the proteome. Cell, 122(6), 957-968.

Stumpf, M. P., Thorne, T., De Silva, E., Stewart, R., An, H. J., Lappe, M., \& Wiuf, C. (2008). Estimating the size of the human interactome. Proceedings of the National Academy of Sciences, 105(19), 69596964.

Tewari, N. (2019). Organic Chemistry-A Modern Approach (Vol. III). McGraw Hill India.

Venkatesan, K., Rual, J. F., Vazquez, A., Stelzl, U., Lemmens, I., Hirozane-Kishikawa, T., ... \& Vidal, M. (2009). An empirical framework for binary interactome mapping. Nature Methods, 6(1), 83-90.

Voet, D., \& Voet J. G. (2010). Biochemistry ( $4^{\text {th }}$ ed.) John Wiley \& Sons, Inc.

Waage, P., \& Gulberg, C. M. (1986). Studies concerning affinity. Journal of Chemical Education, 63(12), 1044.

Wells, J. A., \& McClendon, C. L. (2007). Reaching for high-hanging fruit in drug discovery at protein-protein interfaces. Nature, 450(7172), 1001-1009.

Zhang, G., Andersen, J., \& Gerona-Navarro, G. (2018). Peptidomimetics targeting protein-protein interactions for therapeutic development. Protein and Peptide Letters, 25(12), 1076-1089. 


\title{
Metal Organic Framework: A Future Tool for Nano- Catalysis and Drug Delivery
}

\author{
Prince Saha, Srijita Bhowmik, Supriya Mondal, Suchandra Chatterjee* \\ Department of Chemistry, Surendranath College, Kolkata
}

*Corresponding Author's Email: chatterjeesuchandra01@gmail.com

\begin{abstract}
Metal Organic Frameworks or MOFs are organic-inorganic combined porous and permeable substances. They are made up of metallic ions and organic ligands. Ligands are ions/molecules that are connected to the metal through coordination bonds. During the last two decades MOFs have acquired huge consideration because of their different attributes including high porosity, large surface area, controllable pore sizes, and so on. Current review presents a comprehensive study on different methodologies for synthesis of those MOFs that have higher capacity, rigidity, durability, and ecofriendly nature. This article additionally presents and depicts a few uses of such MOFs like gas storage, nano-catalysis, bioimaging, drug delivery and so forth featuring their tremendous possibilities and enormous prospects.
\end{abstract}

\section{Keywords: Metal Organic Framework; Nano-Porous Material; Building Unit; Drug Delivery; Catalysis; de nova synthesis}

\section{INTRODUCTION}

Metal Organic Frameworks (MOFs) are certain compounds containing metal ions and organic linkers forming a coordinated-organic clusters. Basically, MOFs have originated from coordination polymers (inorganic chemicals) which are porous crystalline materials in which metal is locked into a position by different organic groups (e.g., benzene-1, 4-diamine) to eventually create a rigid as well as porous geometry. They have void spaces in their organic linkers providing structural flexibility, and huge surface region (e.g., $6000 \mathrm{~m}^{2} / \mathrm{gm}$ ) and because of these properties, MOFs have wide range usage in various sectors like gas adsorption and storage, non-linear optics, luminescence, molecular recognition, drug delivery etc. These coordination polymers have different dimensional aspects 1D/2D/3D for which MOFs are useful materials for separation or conversion of molecules as desired. MOFs can likewise be utilized in gas purification, gas separation, in catalysis, and can go about as supercapacitors. In this review article, we have attempted to incorporate recent advances of MOFs, for further exploring and investigating its potential future possibilities.

\section{LITERATURE REVIEW}

\section{(A) Structural Aspects}

Primary Building Units: A primary structural unit is the base where we can find how a MOF is formed. When metal ions connect the organic polymers, they do form a large structural unit resulting in a porous 3D framework. $\mathrm{Cr}^{3+}, \mathrm{Fe}^{3+}, \mathrm{Co}^{2+}, \mathrm{Zn}^{2+}$ are some transition series metal ions that are preferably used in this process. Some s-block elements, Nitrates, Acetates, Sulphates, Chlorides, and Oxides of Lanthanide (rare earth) metals are also used for preparing these MOFs. The organic linkers by which the metals ions remain connected together desired to have functional groups like amine, phosphate, nitrile, carboxylate, sulfonate, etc. that can create coordination bonds.

Secondary Building Units: These SBUs are the main interesting area that helps to build periodic networks that are porous by linking ligands of polytopic organic origin. In these SBUs, Metal-OxygenCarbon clusters connect the organic linkers in place of metal ions. SBUs have intrinsic geometric properties, which facilitate MOF's topology (Yaghi et al. 2003). Nowadays alteration of SBU is an interesting topic by using diverse methodologies such as cation exchange, oxidation state change, defect generation, coordinative incorporation etc. 


\section{(B) Classification}

MOFs can be classified into four main categories, based on their structural features (Sabouni, 2013). They are: Rigid frameworks (Dincă \& Long, 2005), Flexible/dynamic frameworks (Schneemann et al. 2014), Open metal site (Liang, Marshall \& Chaffee, 2009) and Surface functionalized framework (Millward \& Yaghi, 2005).

\section{(C) Synthesis}

In the last two decades, various efforts have been made to synthesize diverse types of MOFs. Organic and inorganic linkers with assorted designs and functionalities have been effectively integrated. Isolated metal ions and organic linkers so far have been synthesized using either of the solvothermal and hydrothermal conditions by means of traditional electrical heating. For speeding up the crystallization and production of uniform diminished sized crystals, several other methodologies have also been tried. A few such procedures are briefly examined beneath:

a) Solvothermal Synthesis: MOFs can be synthesized by this solvothermal process using traditional electrical heating. Here, a mixture of organic linkers and metal ions in a solvent (if water is used then it is called hydrothermal) is heated in a glass apparatus (for lower temperature). Here temperature is an important parameter which is intelligently controlled for obtaining specific structure. If the reaction temperature is found higher than the solvent boiling temperature, the process is alluded as solvothermal and when that temperature is lower than the boiling point, it is called a non-isothermal process. The first two manufactured novel MOFs are HKUST-1 and MOF-5 (Tranchemontagne, Hunt \& Yaghi, 2008) as shown in figure 1.

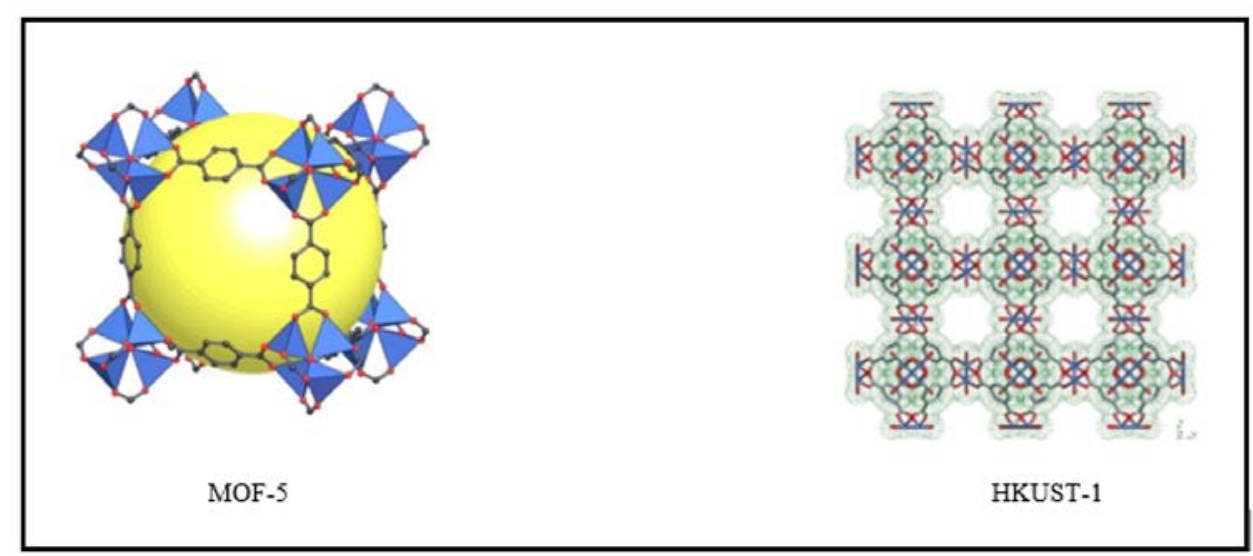

Figure 1: First Two Manufactured Novel MOFs : HKUST-1 and MOF-5

Crystallization rate, particle size and morphologies can have a significant impact on the properties of these MOFs. As for example, in synthesis of MOF-5 if we vary the starting reagent M4( $\mu 4-O)(O A c) 6)$ by changing the metal (M) with Zn/Be, (MOF-5(Zn) and MOF-5(Be) are formed, respectively.

b) Microwave-Assisted Synthesis: In this method, microwave radiation has been used for formation of MOFs. Several MOFs containing $\mathrm{Fe}^{3+}, \mathrm{Al}^{3+}, \mathrm{Cr}^{3+}, \mathrm{V}^{3+}, \mathrm{Ce}^{3+}$ have been synthesized using $\mathrm{MW}$ aided method. Cr-MIL-100 is the first MOF which has been synthesized via MW method (Jhung, Lee \& Chang, 2005). For MW-assisted synthesis to be effective, interaction between the microwave and the polar solvent molecule's electric charge is crucial. High efficiency, particle size reduction, phase selectivity, and control over morphology are some of the major advantages of this method (Jhung et al. 2004; Hwang et al. 2005). MOFs can be generated by this method in noticeably brief time unlike traditional solvothermal technique requiring several days as crystallization time and reduced surface area. CPM- 5 is the MOF which was produced by this method in about 10 minutes. These MOFs can be utilized intelligently for effective separation of carbon dioxide from the flue gas, utilizing their selective $\mathrm{CO}_{2}$ adsorption capability over nitrogen.

c) Electrochemical Synthesis: Researchers at BASF (the famous chemical company) first reported 
the synthesis of MOF using this electrochemical route. They developed new synthesis procedures for formulating some new MOFs using Mg, $\mathrm{Cu}, \mathrm{Zn}$, Co etc. as metals and 1,3,5- $\mathrm{H}_{3} \mathrm{BTC}, \mathrm{H}_{2} \mathrm{BDC}, 1,2,3-$ $\mathrm{H}_{3} \mathrm{BTC}, \mathrm{H}_{2} \mathrm{BDC}-(\mathrm{OH})_{2}$ etc. as organic linkers. Anodic dissolution continuously supplies here metal ions that can react with the dissolved organic linkers. A salt that is conducting enough is also used here in the reaction medium for the electrochemical process to take place. Protic solvents are used to avoid deposition of metal on cathode, but $\mathrm{H}_{2}$ is generated in this process (Mueller et al. 2006). At BASF researchers exploited this process for synthesizing MOF HKUST-1, with a definite aim to eliminate anions for mass production of MOFs in 2005. It was also pointed out that the products obtained via electrochemical routes get inferior quality because of insertion of organic linkers as well as conducting salts in the pores during the crystallization process. Stassen et al. (2015) detailed both anodic and cathodic deposition of electrochemical film of UiO-66 with $\mathrm{Zr}$ as the sole metal (refer to figure 2). This attachment of layer of MOF on the $\mathrm{Zr}$ substrate through bridging oxide layer was done by anodic deposition at $383 \mathrm{~K}$. Cathodic depositions also possessed the benefit of wide substrate adaptability. This synthetic strategy showcased patterned deposition capacity and utilization of UiO-66 in a scaled down sorbent trap for various applications like online analytical sampling as well as concentration determination of dilute organic complexes that are inherently volatile.

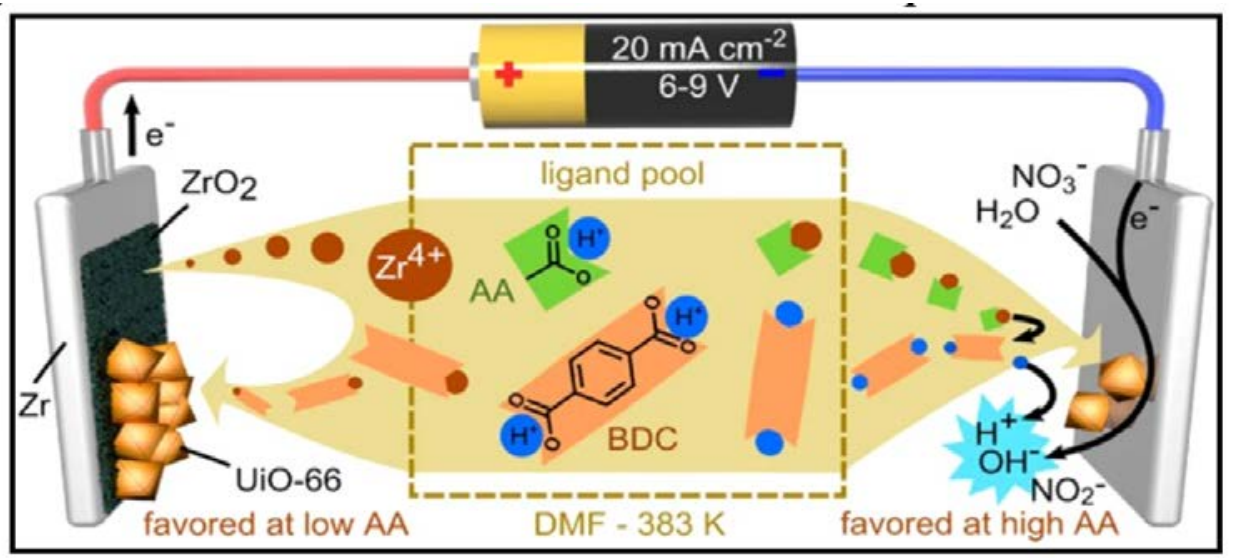

Figure 2: Anodic and Cathodic Electrochemical Deposition Mechanism

d) Sonochemical Synthesis: This is a rapid and environment friendly method in which ultra-sonic radiation is used for MOF synthesis. Contrasted with the traditional procedure of heating, the sonochemical process can achieve homogenous nucleation, decline crystallization time, increase surface area, lower energy utilization, and orchestrate greater portions of nano materials. However, manufactured coloured compounds (azo dye) regularly utilized in textile industry continuously enter the aquatic environment as waste, with escalated harmfulness and shading, they are sure to make natural peril. For removing these dye pollutants from wastewater, adsorption methods by MOFs like 4,4'-oxybis (benzoic acid) (oba) ligand and Zn (II)-based MOFs were proved to be highly effective. Application of Solvothermal and Sonochemical processes in these MOF preparations were extensively tried. Removal efficiency of different MOFs in presence of pollutants like Congo and Sudan red (as shown in figure 3 ) were also investigated in detail.

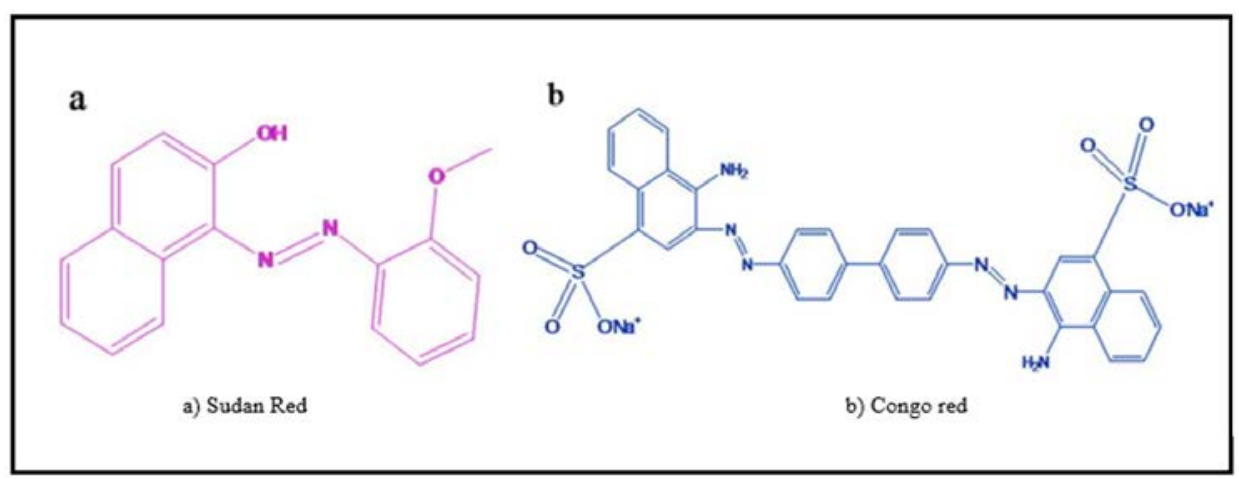

Figure 3: Structures of Congo and Sudan Red 


\section{(D) Factors Affecting Stability of MOFs}

Different promising strategies have been developed in the last twenty years, to modulate stability (particularly chemical) of the MOFs which is vital for their assorted applications in various regimes. Gradual development and production of stable MOFs started with the first ever porous MOF (MOF-5). At the very outset, MOF stability used to normally refer to thermal and mechanical stabilities only. Various MOFs that are stable in water were also reported later based on their structural and chemical reactivity. Even later various methods of crystal growth and crystal structure determination ultimately could generate some extraordinarily stable MOFs, that effectively widened their scope of application and helped in their commercialization too. Imparting of chemical stability to MOFs could be done either by de nova synthesis for new MOF formulation or by improving stability of the existing MOFs. Degradation of such stable MOFs however have been proved to involve metal-ligand bond breaking and formation of products that are more stable compared to them (Antwi-Baah \& Liu, 2018). The chemical stability of MOFs was proved to rely unequivocally upon their characteristic structure, charge density cum coordination number of metal ions and basicity cum hydrophobicity of the participating ligands.

High-Valent Metal containing MOFs: High coordination number of the participating metal ions, strong coordination bond, inertness of metal and so forth are the fundamental requisite for creation of any stable high-valent metal-containing MOF. Those properties obviously help in upgrading the MOF's resilience of hydrolysis before structure breakdown happens. High-valent metals (hard acids) with higher charge density e.g., $\mathrm{Zr}^{4+}, \mathrm{Cr}^{3+}, \mathrm{Al}^{3+}, \mathrm{Fe}^{3+}$ etc. are in general tried to be connected to Oxygen donor ligands (hard bases) to fabricate good MOFs with strong coordination bonding, that as a rule can impart greater chemical stability as per HSAB standard. But the relatively lower pKa values of the carboxylate linkers demand a greater number of such linkers to balance the charges on the central metal ions, thereby resulting in a higher number of metal clusters and a nice degree of acidic stability of the resultant MOFs. F'erey and his group gave birth to a $\mathrm{Cr}$ (III)- based MOF, MIL-101(Cr), with exceptionally large pore sizes and surface area, while Behrens's group (Schaate et al. 2011) developed a unique methodology to regulate the size of crystals of Zr-MOFs from nano to large single crystals, so that it could manifest X-ray diffraction. Needle-shaped single crystals of PCN-222 (Fe), also called MOF-545 and MMPF-6 (Feng D 2012) was formulated by Zhou's group using Fe-tetrakis (4-carboxyphenyl) porphyrin, $\mathrm{Zr}^{4+}$ and benzoic acid as the ligand, metal ion and modulator, respectively. In the synthesis of $\mathrm{Zr}$ based MOF, when the commonest solvent dimethylformamide (DMF) was replaced by a mixture formic acid and acetic anhydride, it yielded MIP-200, with even larger pore sizes that remained stable under extremely harsh conditions, even in concentrated strong acids. Du's group later successfully produced an AI-based MOF (467-MOF) using formic acid as modulator that too showed exceptional stability throughout the $\mathrm{pH}$ range 1 to 11 . Large and good-quality single crystals of another Al-based MOF, AITCS-1 was also formed using Hydrofluoric acid as the modulator, that could remain stable even in aqua regia solution for twenty-four hours. Other tri-valent ions like $\operatorname{~In~}^{3}+$ can also form stable MOFs with $\mathrm{O}$ donor linkers. An unusual out-of-plane porphyrin-based MOF, USTC-8(In) was later synthesized by Jiang's group. Apart from carboxylate, phosphonate, and phenolate-based frameworks also can impart remarkable chemical stability to these high-valent metal-containing MOFs.

Low-Valent Metal containing MOFs: Low-valent metal ions like $\mathrm{Zn}^{2+}, \mathrm{Co}^{2+}, \mathrm{Ni}^{2+}, \mathrm{Fe}^{2+}, \mathrm{Ag}+$ etc. can make hydro-stable skeletons with $\mathrm{N}$ donor linkers with high $\mathrm{pKa}$ values. These metal ions here function as soft acids and get connected to $\mathrm{N}$-containing linkers (soft bases). High pKa values of azoles as organic linkers can help make strong coordination bonds thereby imparting wonderful stability to the resultant MOFs in basic medium. As a rule, the higher is the pKa of the ligand, the steadier are the resultant MOFs in humid conditions. Zn-based Zeolitic imidazolate framework (ZIF)-8 (MAF-4) having zeolite-type topology with imidazolate linkers was found to be stable enough in aqueous as well as in alkaline conditions. Zn-based ZIF series like ZIF-68, 69, 70 also displayed similar stability (Banerjee et al. 2008). Volkmer's group succeeded to fabricate a hydrolytically stable Co-based MOF (MFU-1) (Demessence et al. 2009), that is isostructural with MOF-5 having decent thermodynamical stability for over six months. Further $\mathrm{Ni}$-based ultra-stable framework, $\mathrm{Ni}_{3}(\mathrm{BTP})_{2}$ was also fabricated having 
stability even in boiling aqueous solutions throughout the $\mathrm{pH}$ range 2 to 14 . Self-assembly of lowvalent metal ions with triazole based linkers was also found to form highly stable MOFs. Such a Cubased triazolate-bridged framework Cu-BTTri (Demessence et al. 2009), was reported by Long's group which displayed high tolerance in boiling water and $\mathrm{HCl}$ solution for three days. Another triazolebased system MAF-X27-Cl was also observed to hold its structural uprightness both in acidic and basic environments for more than a week (Lu et al. 2016).

Hydrophobic Ligand Containing MOFs: Hydrophobic properties of MOFs are often tried to be refined by fastening the hydrophobic groups with the organic linkers or by placing them around the metal nodes for reducing their fondness to moisture. For shielding the feeble coordination bonds from possible intervention by water molecules and for boosting the inherent stability of MOFs in aqueous medium, the functional groups that are water repellent are generally arranged around the metal clusters. When methyl groups were attached to MOF-5, H2 uptake capacity of the resultant 2,5dimethyl-MOF-5 was found to be retained. Induction of fluorinated groups ( $-\mathrm{F},-\mathrm{CF}_{3}$ etc.) and other hydrophobic substituents to the organic linkers additionally could improve moisture resistance of the those modified MOFs.

\section{(E) Factors Affecting the Synthesis of MOFs}

Solvents: It is a crucial factor for MOF's synthesis and the solvent utilized can often decide morphology of resultant MOFs. Solvents act either as space-filling agents or they get attached to the metals. The main criteria for being a solvent in MOF synthesis are its high boiling point and polar nature. Dimethylformamide (DMF), dimethylformamide (DEF), dimethylacetamide (DMA), dimethyl sulphoxide (DMSO), alcohols, acetone, acetonitrile, etc. are commonly used as solvents here in this domain. The small difference in the degree of deprotonation of organic linkers in different solvents can result in different morphology of MOFs. It is found that dimensionality of the MOF network and their pore sizes are truly determined by the coordination capacity of solvents with the metal. If we consider some solvents like DMF, DMP, and DMA, the pore size variation will be like DMP>DMA >DMF $(\mathrm{He} Y C$ 2014). (Banerjee et al. 2011) reported MOFs containing magnesium and PDC (3,5- pyridine dicarboxylic acid) have different crystal structure prepared under same condition using different solvents as shown in figure 4. They found that coordination ability of solvent with metal determines the dimensionality of MOF network. Among DMF, $\mathrm{H}_{2} \mathrm{O}$, EtOH and $\mathrm{MeOH}, \mathrm{H}_{2} \mathrm{O}$ has the highest affinity towards $\mathrm{Mg}$ while $\mathrm{EtOH}$ and $\mathrm{MeOH}$ do not have any affinity to coordinate with metal centers and hence $\mathrm{DMF} / \mathrm{MeOH}$ and $\mathrm{EtOH} / \mathrm{H}_{2} \mathrm{O}$ these combinations are used here as potential solvents.

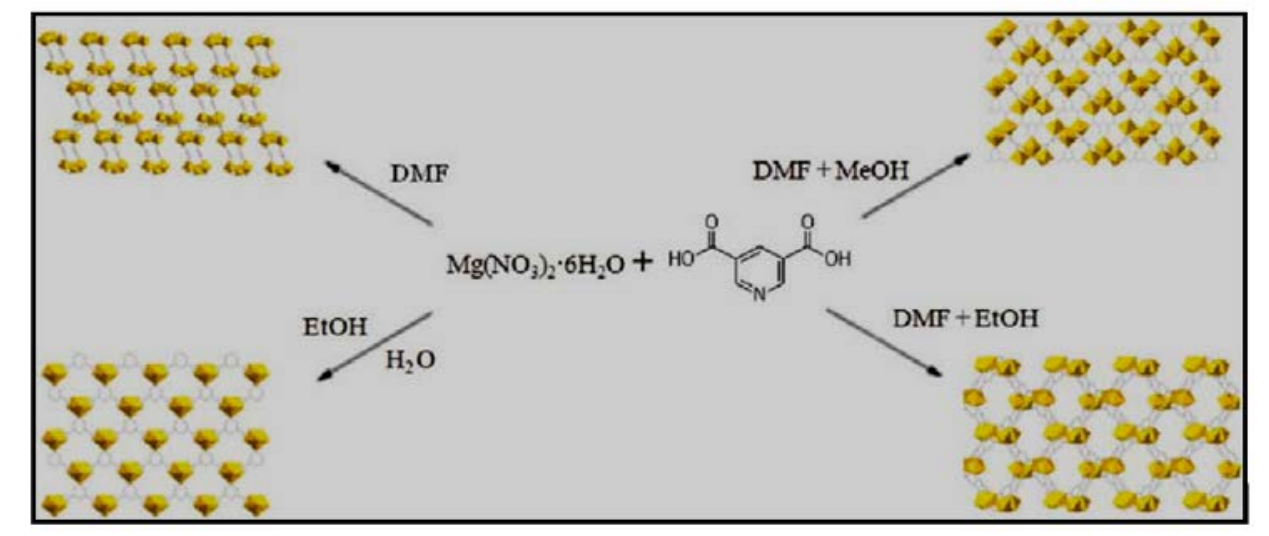

Figure 4: Effect of Solvent Systems on Morphology of MOF

Temperature and $\mathrm{pH}$ : Apart from solvents, other factors that have a significant impact on MOFs are temperature and $\mathrm{pH}$. On expanding the $\mathrm{pH}$ value, the degree of deprotonation of linkers increases (Volkringer et al. 2010). Interpenetrated network has been reported to be formed at greater and lower $\mathrm{pH}$ values and hence moderate $\mathrm{pH}$ is generally preferred. Apart from structure, color and adsorption capability of the MOFs also depends on the $\mathrm{pH}$ of the medium. Compounds with higher dimension are usually formed at higher $\mathrm{pH}$ (Chu et al. 2008). Now coming to temperature, formation of large crystals 
of high quality is often supported by an elevated temperature favoring high solubility of the reactants. Nucleation and growth rates also get affected. Morphology of synthesized MOFs can also be altered by varying the temperature of the medium. MOFs of Tm-succinate having similar empirical formula, but varying morphology (monoclinic, and triclinic) can be synthesized at different temperatures following the same recipe. (Bernini et al. 2007) prepared two Ho-succinate MOFs and reported that the MOFs prepared via hydrothermal method at higher temperature is thermally more stable as compared to the other prepared at room temperature. Less hydrated, denser, and higher dimensional solids with greater thermal stability can be generated by properly utilizing the hydrothermal methods.

\section{DISCUSSION}

\section{Major Applications of MOFs}

In MOF there is a simultaneous appearance of three characteristics viz. crystallinity, porosity, and existence of strong metal-ligand interaction. The unique combination of these properties makes MOFs an incredibly special class of materials (Corma, García \& Llabrés i Xamena, 2010). MOFs show versatile applications in catalysis, drug delivery, gas storage, luminescence, electrochemistry, sensor technology and so forth. Some of those applications are discussed below.

a) Gas Storage: Several manual methods are available there for effective storing of different gases. However, all such techniques generally require high pressure tanks, multistage compressors etc. and hence are not cost friendly for practical uses. Thus, a dire need was always there to develop a simpler and cheaper alternative for this storage of gases. To beat these issues and to discover more secure storage techniques for gases, use of Zeolite like materials or enacted porous carbons have been extensively explored. In this context, MOFs have provided edge over other materials. This is due to their easy preparative methods, higher surface area, wide scope of applications and tunable pore structure and size that has made these MOFs preferred over other available porous materials. Numerous MOFs so far have been tested for $\mathrm{H}_{2}$ storage capacity and $\mathrm{Zn}$-based MOF-177 (Wong-Foy, Matzger \& Yaghi, 2006) was found to be most effective for this purpose. Large surface area ( $\sim 5000$ $\mathrm{m}^{2} \mathrm{~g}-1$ ) and pore volumes helps it in higher gravimetric $\mathrm{H}_{2}$ uptake. Another $\mathrm{Zn}$-based MOF-5 (IRMOF1) and MOF-210, MIL-101, HKUST-1, NU-100, PCN-12, NOTT-102, MOF-205 etc. were found to be effective for this $\mathrm{H}_{2}$ storage. By and large, it was observed that MOFs having metal sites that are open can provide larger surface area establishing more grounded connection between metal ions and molecules of $\mathrm{H}_{2}$. This is no doubt the basic reason behind the higher $\mathrm{H}_{2}$ uptake by all these MOFs. NU100 and MOF-210 were found to have the highest excess $\mathrm{H}_{2}$ storage capacity and highest total $\mathrm{H}_{2}$ storage capacity respectively. In addition, experimentally, it was also reported that metal ion doping of MOFs can enhance the $\mathrm{H}_{2}$ uptake capacity (Dincă \& Long, 2007). As compared to other $\mathrm{H} 2$ storage systems such as transition metal hydrides, MOFs require truly little energy to release the adsorbed hydrogen. This released hydrogen can further be used in automobile and fuel cell industries. For reducing the $\mathrm{CO}_{2}$ level in the atmosphere MOFs have also been proved to be amazingly effective. MOF-210 with its highest surface area (known to date) can uptake huge amount of $\mathrm{CO}_{2}$ exceeding the capacity of any other known porous materials. MOF-200 was also found to possess similar $\mathrm{CO}_{2}$ uptake capacity (Furukawa et al. 2010).

b) Magnetism: MOF materials can show magnetism when paramagnetic property of transition metal ( $\mathrm{V}, \mathrm{Cr}, \mathrm{Mn}, \mathrm{Fe}, \mathrm{Co}, \mathrm{Ni}$ and $\mathrm{Cu}$ ) nodes gets combined with the diamagnetic property of the organic linkers (Coronado \& Espallargas, 2013). MOFs having magnetic properties are generally known as Magnetic Metal Organic Frameworks (MMOFs). Closed-shell ligands such as oxo, cyano, azido bridges and polycarboxylic ligands, that have feeble magnetic interaction, are acceptable for this purpose. Another justification for the magnetic conduct of MMOFs is the inherent system structure, which might include layered geometry with an even more limited distance in between the metal clusters. Organic linkers have likewise been utilized for the synthesis of these MMOFs, where radicals present in the organic linkers do effectively superintend the overall magnetic properties of the MMOFs. This metal-radical joint approach has also been used for synthesizing a variety of MMOFs (Roques et al. 2008a; Roques et al. 2008b). For the environmental application in expulsion of arsenic, magnetic MOFs can likewise be investigated. Arsenic removal by magnetic nanoclusters have been reported 
several times.

c) Nano-Catalysis: The very existence of metal-ligand strong interaction in MOFs is solely responsible for imparting sufficient porosity to the material making complete removal of solvent molecules feasible without any structural collapse. If metal centers are not fully blocked by organic ligands in MOFs, they can often have immense potential to become good heterogeneous catalysts due to generation of a free coordination position on the metal when labile ligands and solvent molecules are removed. HKUST-1 is such an example, in which coordinated water molecule leaves a coordination vacancy on $\mathrm{Cu}$ upon thermal activation (Bordiga et al. 2007). Several organic reactions have been reported till date to be catalyzed by using these nano porous MOFs.

\section{d) Biomedical Application:}

Drug Delivery: Effective drug delivery system that is controlled is no doubt crucial to reduce cost and side effects of any drug thereby enhancing its therapeutic effectiveness. The unique properties of the MOFs; their defined pore size, tailorable composition cum structure, tunable size, improved biocompatibility have made them a suitable candidate as drug delivery hosts. With advanced synthetic application their drug loading ability can be changed also. Zr-MOFs having stability towards both hydrolysis and post synthetic modification with nominal toxicity are very much suitable for these drug delivery applications.

Bio Imaging: MIL-101(Fe) is generally used for bio imaging purposes. Whenever a drug is introduced, we can find out the exact location of the drug molecules using Cr-Carboxylate MOFs, MIL-100 \& 101 (Bernini et al. 2014), exhibiting high drug storage capacities and controlled drug release behaviour.

\section{Future Scope}

Although comprehension about MOFs is developing quickly during the last couple of years however, there are yet critical gaps in our complete understanding of their actual structure, stability, and various observed properties. Detailed investigation on factors responsible for destruction of crystal structure of certain MOFs with time, mechanism of their stability and decomposition have still not been conducted systematically. There are some reports on applications of MOFs in drug loading and delivery for some anticancer and antiviral molecules, though detailed toxicological investigation needs to be conducted before commercialization of such products. Lately, MOFs are becoming progressively pertinent in chemical and synthetic industry too. To prevent production of $\mathrm{CO}_{2}$ at an alarming rate we must upgrade our current infrastructure and depend on renewable resources such as ocean water or $\mathrm{H} 2$ which can be done by using these MOFs. In India, the energy producing industries depends on thermal power plants where coal is the most important fuel, and it is expected that by 2050 all of us again must have to wear masks not for the corona virus but for the $\mathrm{CO}_{2}$ pollution and other hazardous gases in air. Though our understanding about MOFs is developing quickly during late couple of years however there are yet critical limits to the acquired knowledge. Pushing ahead, critical examination of mechanisms and gadget designing would be proved as additionally appealing objectives for creating stabler and effective MOFs in future.

\section{CONCLUSION}

In recent many years, much exertion has been committed to de nova synthesis for preparing novel, stabler MOFs, beginning from high-valent metal carboxylate to low-valent metal-azolate MOFs. In chemical stability examination of MOFs, their stability in water or moisture was found to be most important and critical. This is because dealing with MOFs in air without moisture is not practically possible. Luckily, generous number of MOF-based materials that are steady both in air and water have already been formulated till date. Additionally, expanded number of MOFs that are safe even to harsh chemical conditions (acidic/soluble media) have likewise been developed too. AITCS-1 can remain stable and intact even in aqua regia medium more than 24 hours. Similarly, PCN-601 also does not get upset even when immersed in concentrated $\mathrm{NaOH}$ at $100^{\circ} \mathrm{C}$. These significant forward leaps along with numerous other advances provide the MOF community incredible support and certainty. The stability (particularly chemical) of MOFs, which was once viewed as a basic bottleneck for their 
certifiable applications, may at this point do not be an extreme trouble and ought to get settled soon with the persistent endeavors of researchers in this field. As above and beyond, in view of the intriguing properties of these MOFs, the extent of their applications has also essentially extended. Here, in this brief review, a couple such MOFs along with their applications have been covered. Without any doubt, the emergence of more improved and stabler MOFs and their connection with other fields of research, will bring out really encouraging and more creative applications of them in not-so-distant future.

\section{ACKNOWLEDGEMENT}

The authors express their sincere thanks to Department of Biotechnology, Government of India for the funding from DBT-STAR College grant, under which this review project was conducted. They are also grateful to the Principal and the DBT-STAR Coordinator, Surendranath College, for their support and encouragement in implementing this review project at undergraduate level.

\section{REFERENCES}

Antwi-Baah, R., \& Liu, H. (2018). Recent hydrophobic metal-organic frameworks and their applications. Materials, 11(11), 2250.

Banerjee, D., Finkelstein, J., Smirnov, A., Forster, P. M., Borkowski, L. A., Teat, S. J., \& Parise, J. B. (2011). Synthesis and structural characterization of magnesium-based coordination networks in different solvents. Crystal growth \& design, 11(6), 2572-2579.

Banerjee, R., Phan, A., Wang, B., Knobler, C., Furukawa, H., O'Keeffe, M., \& Yaghi, O. M. (2008). Highthroughput synthesis of zeolitic imidazolate frameworks and application to $\mathrm{CO} 2$ capture. Science, 319(5865), 939-943.

Bernini, M. C., Brusau, E. V., Narda, G. E., Echeverria, G. E., Pozzi, C. G., Punte, G., \& Lehmann, C. W. (2007). The Effect of Hydrothermal and Non-Hydrothermal Synthesis on the Formation of Holmium (III) Succinate Hydrate Frameworks.

Bernini, M. C., Fairen-Jimenez, D., Pasinetti, M., Ramirez-Pastor, A. J., \& Snurr, R. Q. (2014). Screening of bio-compatible metal-organic frameworks as potential drug carriers using Monte Carlo simulations. Journal of Materials Chemistry B, 2(7), 766-774.

Bordiga, S., Regli, L., Bonino, F., Groppo, E., Lamberti, C., Xiao, B., ... \& Zecchina, A. (2007). Adsorption properties of HKUST-1 toward hydrogen and other small molecules monitored by IR. Physical Chemistry Chemical Physics, 9(21), 2676-2685.

Chu, Q., Liu, G. X., Okamura, T. A., Huang, Y. Q., Sun, W. Y., \& Ueyama, N. (2008). Structure modulation of metal-organic frameworks via reaction $\mathrm{pH}$ : Self-assembly of a new carboxylate containing ligand $\mathrm{N}$-(3-carboxyphenyl) iminodiacetic acid with cadmium (II) and cobalt (II) salts. Polyhedron, 27(2), 812-820.

Corma, A., García, H., \& Llabrés i Xamena, F. X. (2010). Engineering metal organic frameworks for heterogeneous catalysis. Chemical reviews, 110(8), 4606-4655.

Coronado, E., \& Espallargas, G. M. (2013). Dynamic magnetic MOFs. Chemical Society Reviews, 42(4), 1525-1539.

Demessence, A., D’Alessandro, D. M., Foo, M. L., \& Long, J. R. (2009). Strong CO2 binding in a waterstable, triazolate-bridged metal- organic framework functionalized with ethylenediamine. Journal of the American Chemical Society, 131(25), 8784-8786.

Dinca, M., \& Long, J. R. (2005). Strong H2 binding and selective gas adsorption within the microporous coordination solid Mg3 (O2C-C10H6-CO2) 3. Journal of the American Chemical Society, 127(26), 9376-9377.

Dinca, M., \& Long, J. R. (2007). High-enthalpy hydrogen adsorption in cation-exchanged variants of the 
microporous metal- organic framework Mn3 [(Mn4Cl) $3(\mathrm{BTT}) 8(\mathrm{CH} 3 \mathrm{OH})$ 10] 2. Journal of the American Chemical Society, 129(36), 11172-11176.

Furukawa, H., Ko, N., Go, Y. B., Aratani, N., Choi, S. B., Choi, E., ... \& Yaghi, O. M. (2010). Ultrahigh porosity in metal-organic frameworks. Science, 329(5990), 424-428.

Hwang, Y. K., Chang, J. S., Park, S. E., Kim, D. S., Kwon, Y. U., Jhung, S. H., ... \& Park, M. S. (2005). Microwave fabrication of MFI zeolite crystals with a fibrous morphology and their applications. Angewandte Chemie International Edition, 44(4), 556-560.

Jhung, S. H., Chang, J. S., Hwang, Y. K., \& Park, S. E. (2004). Crystal morphology control of AFI type molecular sieves with microwave irradiation. Journal of Materials Chemistry, 14(2), 280-285.

Jhung, S. H., Lee, J. H., \& Chang, J. S. (2005). Microwave synthesis of a nanoporous hybrid material, chromium trimesate. Bulletin of the Korean Chemical Society, 26(6), 880-881.

Liang, Z., Marshall, M., \& Chaffee, A. L. (2009). CO2 adsorption-based separation by metal organic framework (Cu-BTC) versus zeolite (13X). Energy \& Fuels, 23(5), 2785-2789.

Lu, X. F., Liao, P. Q., Wang, J. W., Wu, J. X., Chen, X. W., He, C. T., ... \& Chen, X. M. (2016). An alkalinestable, metal hydroxide mimicking metal-organic framework for efficient electrocatalytic oxygen evolution. Journal of the American Chemical Society, 138(27), 8336-8339.

Millward, A. R., \& Yaghi, O. M. (2005). Metal- organic frameworks with exceptionally high capacity for storage of carbon dioxide at room temperature. Journal of the American Chemical Society, 127(51), 17998-17999.

Mueller, U., Schubert, M., Teich, F., Puetter, H., Schierle-Arndt, K., \& Pastre, J. (2006). Metal-organic frameworks - prospective industrial applications. Journal of Materials Chemistry, 16(7), 626-636.

Roques, N., Maspoch, D., Imaz, I., Datcu, A., Sutter, J. P., Rovira, C., \& Veciana, J. (2008). A threedimensional lanthanide-organic radical open-framework. Chemical communications, (27), 31603162.

Roques, N., Maspoch, D., Luis, F., Camón, A., Wurst, K., Datcu, A., ... \& Veciana, J. (2008). A hexacarboxylic open-shell building block: synthesis, structure, and magnetism of a three-dimensional metal-radical framework. Journal of Materials Chemistry, 18(1), 98-108.

Sabouni, R. (2013). Carbon dioxide adsorption by metal organic frameworks (Synthesis, Testing and Modeling).

Schaate, A., Roy, P., Godt, A., Lippke, J., Waltz, F., Wiebcke, M., \& Behrens, P. (2011). Modulated synthesis of Zr-based metal-organic frameworks: from nano to single crystals. Chemistry, 17(24).

Schneemann, A., Bon, V., Schwedler, I., Senkovska, I., Kaskel, S., \& Fischer, R. A. (2014). Flexible metal-organic frameworks. Chemical Society Reviews, 43(16), 6062-6096.

Stassen, I., Styles, M., Van Assche, T., Campagnol, N., Fransaer, J., Denayer, J., ... \& Ameloot, R. (2015). Electrochemical film deposition of the zirconium metal-organic framework UiO-66 and application in a miniaturized sorbent trap. Chemistry of materials, 27(5), 1801-1807.

Tranchemontagne, D. J., Hunt, J. R., \& Yaghi, O. M. (2008). Room temperature synthesis of metal-organic frameworks: MOF-5, MOF-74, MOF-177, MOF-199, and IRMOF-0. Tetrahedron, 64(36), 8553-8557.

Volkringer, C., Loiseau, T., Guillou, N., Ferey, G., Haouas, M., Taulelle, F., ... \& Stock, N. (2010). Highthroughput aided synthesis of the porous metal- organic framework-type aluminum pyromellitate, MIL-121, with extra carboxylic acid functionalization. Inorganic chemistry, 49(21), 9852-9862.

Wong-Foy, A. G., Matzger, A. J., \& Yaghi, O. M. (2006). Exceptional H2 saturation uptake in microporous metal- organic frameworks. Journal of the American Chemical Society, 128(11), 3494-3495.

Yaghi, O. M., O'Keeffe, M., Ockwig, N. W., Chae, H. K., Eddaoudi, M., \& Kim, J. (2003). Reticular synthesis and the design of new materials. Nature, 423(6941), 705-714. 


\title{
Artificial Molecular Machines with Bio-inspired Mechanisms of Natural Molecular Devices: An Overview
}

\author{
Arghadeep Sarkar, Suchandra Chatterjee* \\ Department of Chemistry, Surendranath College, Kolkata, India \\ *Corresponding Author's Email: chatterjeesuchandra01@gmail.com
}

\begin{abstract}
The investigation of Molecular Machines has acquired gigantic fame of late, inferable from its wide acknowledgment on granting the Noble Prize in Chemistry in 2016 to Sauvage, Stoddart and Feringa for their monstrous commitment in this field. This progress is attributed to the invention of modern techniques that have enabled a better observation on nanoscale objects and to a better understanding of intermolecular and intramolecular interactions and mechanisms of different biochemical processes. A molecular machine is defined as an assembly of molecular components that are interlocked to bring certain movement or conversion of energy. These machines can be controlled by making alteration in the external environment. In this review, a discussion has been made on natural molecular devices especially the motor proteins along with some basic artificial molecular machines like rotaxanes and catenanes. Discussion is also done on the mechanism i.e., how these special molecules are made to work by stimulating external conditions.
\end{abstract}

\section{Keywords: Molecular Machine; Natural Molecular Device; Artificial Molecular Machine; Rotaxane; Catenane}

\section{INTRODUCTION}

The living world is entirely governed by diverse types of movement, and it is the movement of molecules that is responsible for every biochemical process that make up an organism. The world that we see today is a consequence of the movement of organisms and the growth of human lifestyle that is attributed in turn to the construction and development of advanced machinery. Hence, discussion about movement is essential for a better understanding of this topic. In physics, a machine is always defined as a combination of 'moving' parts which together can function as a force multiplier or can change the direction of force in a desired direction or can function as a speed multiplier. In 20th century, the advancement in technology is primarily focused on miniaturizing of those machines. Also, there has been outstanding progress in the field of molecular biology due to inventions of numerous powerful techniques like single-molecule fluorescence spectroscopy (Rigler, Orrit, \& Basché, 2012) and various microscopic analysis that can help to determine the shape, size of nanoscale objects. This led to a new thinking of applying this miniaturizing concept at molecular level to open innovative technologies in the field of energy, environment, and medical sciences. However, performing an operation using molecular devices needs a firm control over the constitution of molecules, which involves a precise work of construction of molecules through a 'bottom-up' approach. Chemists have always preferred molecules instead of atoms as convenient parts for construction of these nanoscale devices and machines, on account of their independent existence and exact shape unlike atoms. The movement of the components of molecular machines implies that chemical reactions are taking place. Richard Feynman, Nobel Laureate in Physics, the first person to have made serious introspections on this field (Feynman, 1960) noted in his 1959 address to the Royal Physical Society that "An internal combustion engine of molecular size is impossible. Other chemical reactions, liberating energy when cold, can be used instead." However, even relatively cold chemical reactions can also destroy the constituents of the machine. The electronic or nuclear rearrangements make these molecules work and are characterized by the type of energy applied to them to make them work. The motion of these parts is 
due to the rotation around a covalent bond or breaking and making of non-covalent bonds that are caused by the chemical reactions which are preferably reversible since these machines usually function in repeating cycles. However, construction of such molecular machines is as complex as those found naturally in ATP synthase, myosin, DNA synthase, kinase, ribosome etc. Construction of such kind of machines appear to be almost next to impossible. Hence, the molecular devices that are constructed artificially are comparatively much simpler. These devices are employed to perform functions like catalysis and transportation in natural systems. These artificial devices are known to perform logical operations also, like information processing. In 2016, the Nobel Prize in Chemistry was awarded to three organic chemists, Jean-Pierre Sauvage, J. F. Stoddart, and Ben Feringa for the design and synthesis of these molecular machines. They made huge contributions for almost three decades towards establishing ways of exploiting chemical energy to do mechanical work (Sauvage, 1998, Balzani et al. 1998, Browne \& Feringa, 2010). Often electrical and light energy are used to induce these mechanical movements, which gradually led to creation of molecular switches, valves, elevators, actuators, etc. later (Heard \& Goldup, 2020).

\section{DISCUSSION}

\section{A. Natural Molecular Machines}

Prior to examining about artificial molecular machines that do not occur naturally, let us talk about the natural machines at the very outset. A living organism itself is the most complex machine because of existence of billions of component machines (which are not mandatorily microscopic) that work together to make an organism work ultimately as a whole. As stated earlier, it is impossible to reproduce these naturally occurring devices artificially. One of the main functions of natural molecular machines is to convert energy from one form to other. Most of the molecular machines found in nature are protein based. These proteins are employed to perform various tasks which include catalysis of various reactions in biological system, functioning as enzymes, transmission of information within cells, etc. The powerful crystallographic techniques have enabled us to get a detailed understanding about the structure and conformation of those proteins. A few such examples are given below.

(i) Fo $F_{1}$-ATP Synthase: Adenosine triphosphate (ATP) as shown in figure 1 is the energy currency for all biological processes. It is a compound of high-energy potential that acts as a transducer of energy from a wide range of exergonic reactions to an equally wide range of endergonic reactions or processes, such as biosynthesis, muscular contraction, nervous excitation, and active transport. ATP consists of a nucleotide having three phosphate molecules that are labelled as $\alpha, \beta$ and $\gamma$ in order of their proximity with the ribose sugar of the nucleotide and stores much energy in its phosphate groups (Knowles \& Penefsky, 1972, Lipmann, 2013). In its reactions in the cell, it functions as a $\mathrm{Mg}^{2}+$ complex. When ATP is used to supply energy to any biochemical process, it simply dissociates to ADP and a phosphate group to ultimately release energy.

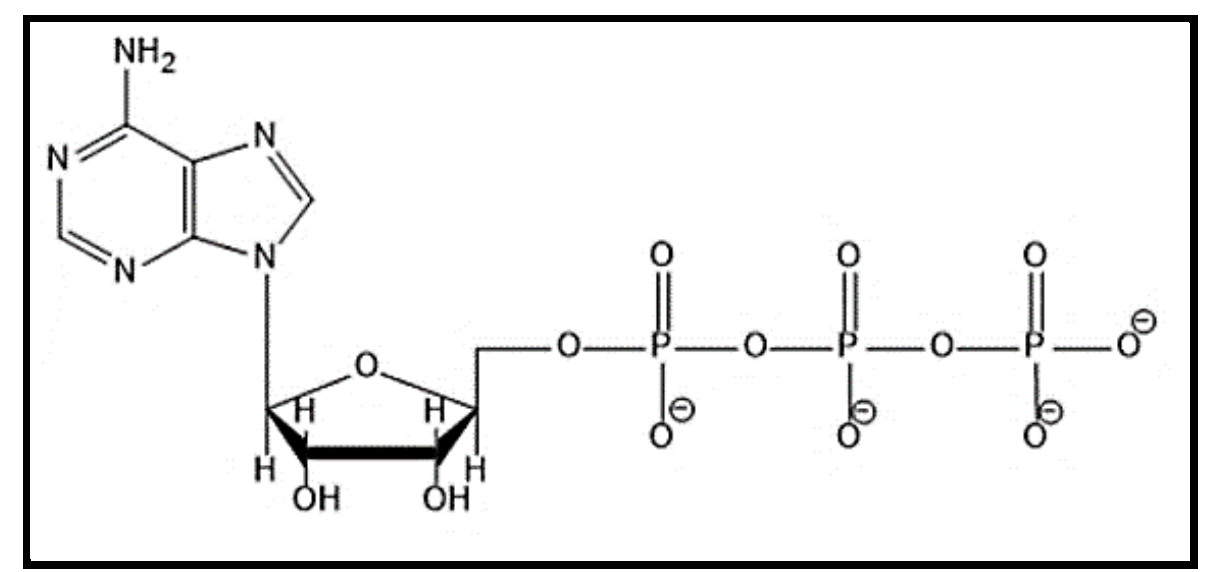

Figure 1: Structure of ATP 
ATP synthase as shown in figure 2 is basically a mitochondrial enzyme present in the inner mitochondrial membrane that catalyses the ATP synthesis (Sielaff et al., 2008). It is a combination of two motors functioning together: the $F_{0}$ motor and $F_{1}$ motor. The $F_{0}$ motor, present in the inner mitochondrial membrane, is composed of 'a', 'b' and 'c' subunits. There is one 'a' subunit which forms the proton channel, two 'b' subunits that form a peripheral stalk when connected to the $F_{1}$ region and ten subunits of ' $c$ ' which together forms a ring that rotates the $\gamma$ subunit of $F_{1}$ (Jones \& Fillingame, 1998). The $F_{1}$ portion is the catalytic region composed of 5 subunits; a $\gamma$ subunit which is the central stalk that connects $F_{0}$ with $F_{1}$, alternating subunits of $3 \alpha$ and $3 \beta$ are also arranged to make a hexamer ring having a circular structure that binds the ADP and Pi molecules and catalyse the synthesis of ATP, a $\delta$ subunit is there to hold the structure together and a $\varepsilon$ unit also, that attaches the $Y$ unit with $\mathrm{C}-10$ unit. The Fo portion exists within the mitochondrial membrane across which there exists a proton concentration gradient. The protons travel across the membrane through $F_{0}$ and thereby create a torque between the $a$ and $c$ subunits, like a turbine. The torque is then transmitted through the $y$ and $\varepsilon$ subunit to $F_{1}$. $F_{1}$ basically acts as an assembly unit that takes up ADP and Pi floating in the solution and combine them into ATP and finally releases it (Wang \& Oster, 1998).

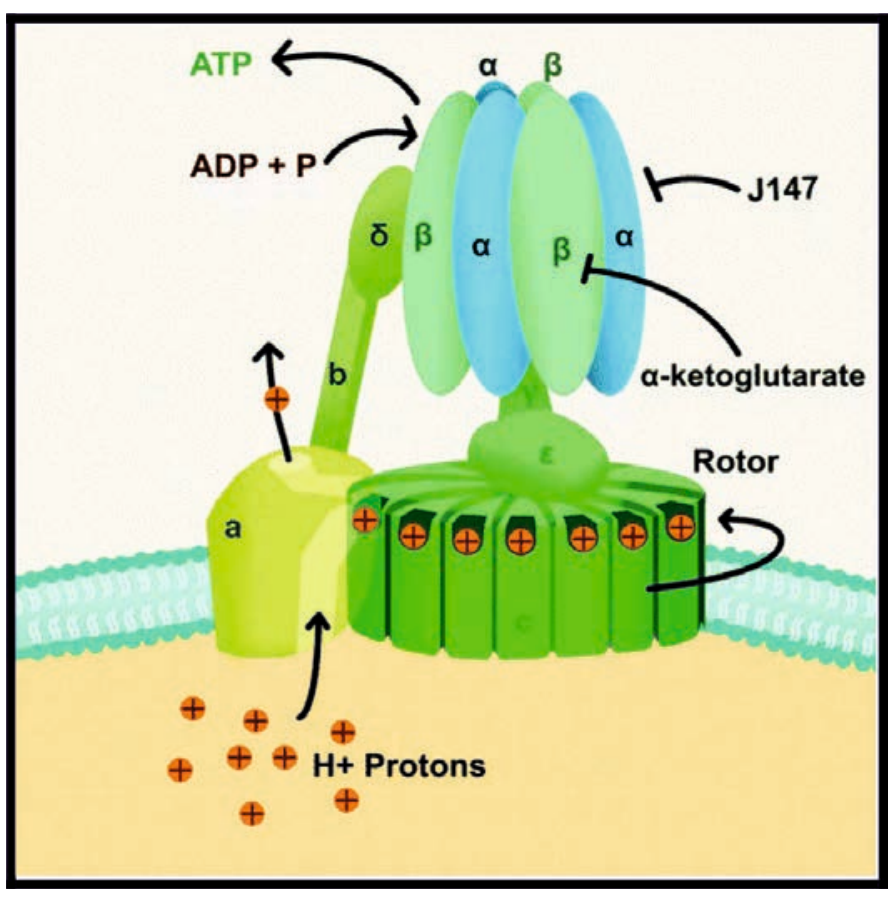

Figure 2: Working mechanism of F0F1-ATP synthase (Larrick, Larrick \& Mendelsohn, 2018)

(ii) Motor Proteins: The motor proteins use the energy acquired by ATP hydrolysis to perform mechanical work. There are three known motor proteins- kinesin, dynein and myosin that play significant role in cell division and motility (Katchalsky, 2003) as shown in figure 3. Before we discuss

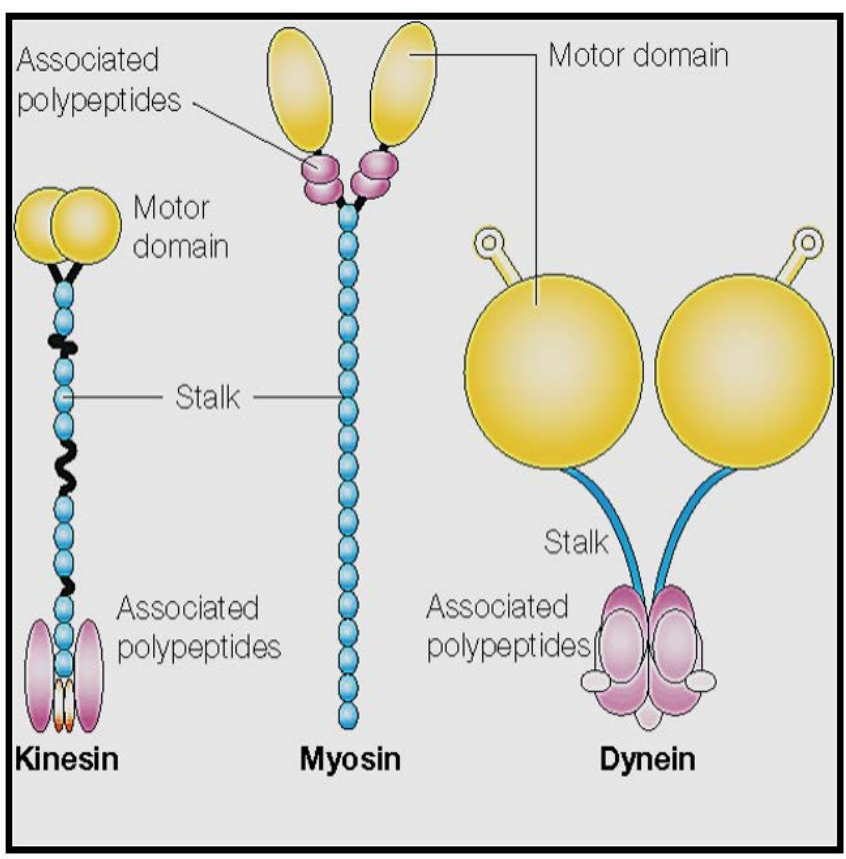

Figure 3: Labelled diagrams for different motor proteins (Le et al. 2019) about motor proteins, we must know about the microtubules. Microtubules is one of the three cytoskeleton fibres that play key role in figuring out the cell shape and cell motility. It is basically a polymer of a heterodimer tubulin protein having a hollow, cylindrical structure. The microtubules have two polar ends- one positive and the other negative (Howard et al., 1989). Kinesin moves from the negative end to the positive end of the microtubule while dynein move in the opposite direction. The microtubules create a track along which the motor proteins "walk," carrying different organelles, cargo, and vesicles (Endow, 2003). Now let us discuss some length about some motor proteins in details.

(a) Kinesin: The kinesin family was discovered in 1985 and it is the smallest molecular motor discovered so far. Analysis of its crystal structure has enabled us to know about its structural information. It is basically a stalk made up of two 
long chains that bind with the microtubules through two globular head region and has a tail that carries the cargo. The kinesin motor takes numerous steps carrying the cargo by forwarding it from one head to another. At the initial stage of movement, each of the two heads carries an ADP molecule and the movement is initiated as a head loses ADP to have a stronger binding with microtubules. As the ADP is lost, ATP enters the empty nucleotide binding site thus leading to a conformational change that snaps the neck region forward and the other head carrying the ADP binds to the microtubules. The former head that carries the ATP now hydrolyses the ATP to release the Pi and now this head is left with an ADP as in the initial stage. The same process is repeated and again and this coordinated ATP hydrolysis of the two motor heads ultimately results in a movement (Block, 2007). A kinesin motor takes about one hundred steps before detaching itself from the microtubule that has a speed of about $100 \mathrm{~nm} / \mathrm{s}$.

(b) Dynein: Another motor protein that transports vesicles along the microtubules is the dynein superfamily (Gibbons \& Rowe, 1965). Like Kinesin, it also has two head portions, but three intermediate chains and four lighter intermediate chains. Dynein is a more complex motor than Kinesin and thus its way of transport is not still known in detail but certainly that would be different from that of kinesin since it belongs to the AAA superfamily of mechanoenzymes.

(c) Myosin: Myosin is another superfamily of motor proteins that converts energy of ATP into mechanical work, i.e., it pulls the Actin filament to the required destination to conduct voluntary motion as well as involuntary muscle contraction (Citi \& Kendrick-Jones, 1987). It refers to fourteen classes of proteins each having an Actin-based motor. Myosin is composed of two polypeptide chains that contain two globular head region which is the binding site for Actin and ATP (Weeds \& Lowey, 1971). There is a neck region formed from the polypeptide chain and the two chains coil around each other to form the tail region. The tails of Myosin associate to form a thick bundle from where the heads protrude out. When ATP binds with the head region that was initially connected to the Actin subunit of the filament, a change in configuration occurs, making the heads bound to release the chain. The ATP then hydrolyses to ADP and $\mathrm{Pi}$ that results in a conformational change again and the lever arm swings to bind with the Actin further along the filament. As the myosin head binds, the ADP and Pi is released, and Myosin returns to its initial position (Wagner \& Giniger, 1981). The repetition of all these steps in the form of a mechanochemical cycle ultimately causes the thin actin filament to move forward and this action is known as the power stroke.

\section{B. Artificial Molecular Machines}

Artificial molecular machines are constructed by molecules of several kinds, as well as supramolecular systems. These devices so produced are aimed to be able to store, receive and process information. The recent progress of chemical synthesis of supramolecules and the non covalent bonds has helped in templating reactions to develop these machines. However, these devices are composed of structures based on rotaxanes and catenanes. These structures are the fundamental building blocks of different nanoscale devices and are regarded as molecules rather than supramolecules although the components are not covalently bonded (Lehn, 1996).

(i) Rotaxane: The name rotaxane is derived from the Latin words 'rota' and 'axis' which means wheel and axle and the word catenane is derived from 'catena' which means chain. A rotaxane is a mechanically interlocked molecular structure composed of a dumb-bell shaped part, a rod section and a macrocyclic ring as shown in figure 4 .

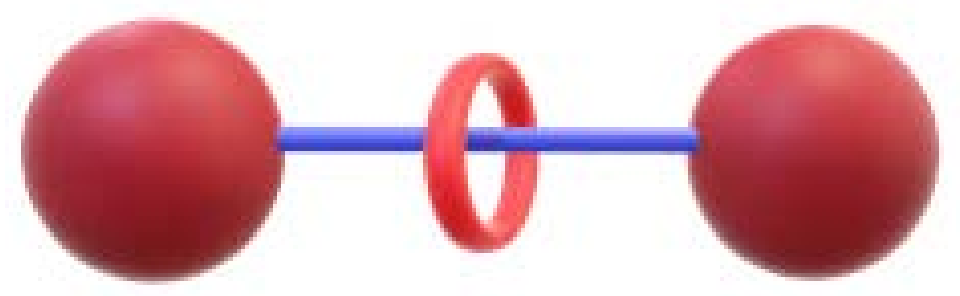

Figure 4: Schematic Representation of Rotaxane 
The constituents of rotaxanes are adjusted such that the diameter of the ring is lesser than the two bulky stoppers attached at both ends of the rod-like portion and hence the two components are mechanically trapped (Balzani, Credi \& Venturi, 2003). Two types of movement can be brought about by this structure (i) shuttling of the macrocyclic ring along the axle, and (ii) rotation of the ring along the axis. This shuttling motion has been used in different molecular motors and molecular elevators. There is another known form of rotaxane where the macrocyclic ring may be large enough to come out of the rod.

(ii) Catenane: Catenane is another supramolecular entity in which two or more macrocyclic rings are interlocked (as shown in figure 5) where no chemical interaction necessarily exists between the rings (Balzani, Credi \& Venturi, 2003). The

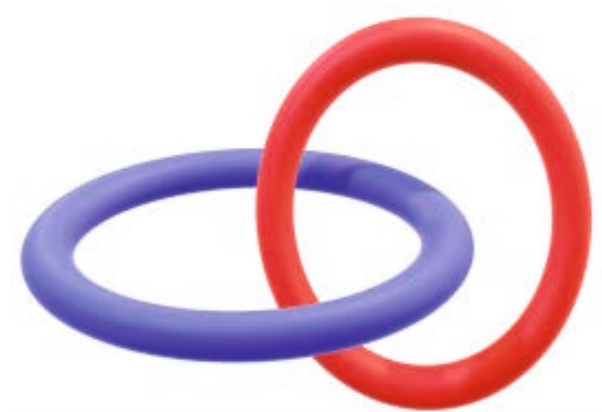

Figure 5: Schematic Representation of Catenane seperation of the constituent rings involves disruption of the rings of the macrocycle and these rings are held together by mechanical bonds (Schill \& Lüttringhaus, 1964). These catenane units are used to design molecular switches, molecular sensors and may work as molecular information processing devices (Balzani, Credi \& Venturi, 2003).

The stability of these artificially derived molecular machines is determined by interaction within their different components. These interactions involve electron donor-acceptor property, $\pi-\pi$ stacking, hydrophobic-hydrophilic interaction, hydrogen bonding, etc. These interactions can be manipulated by external means which serve as a way of controlling these devices. The components of these devices are trapped via non-covalent bonds. Hence, the mechanical movements mostly take place between two different well-defined states as the molecules reversibly isomerise between two stable structures according to the external conditions and are accompanied by on/off switching of some observable properties as manifested in their NMR or electronic absorption spectra or in luminescence.

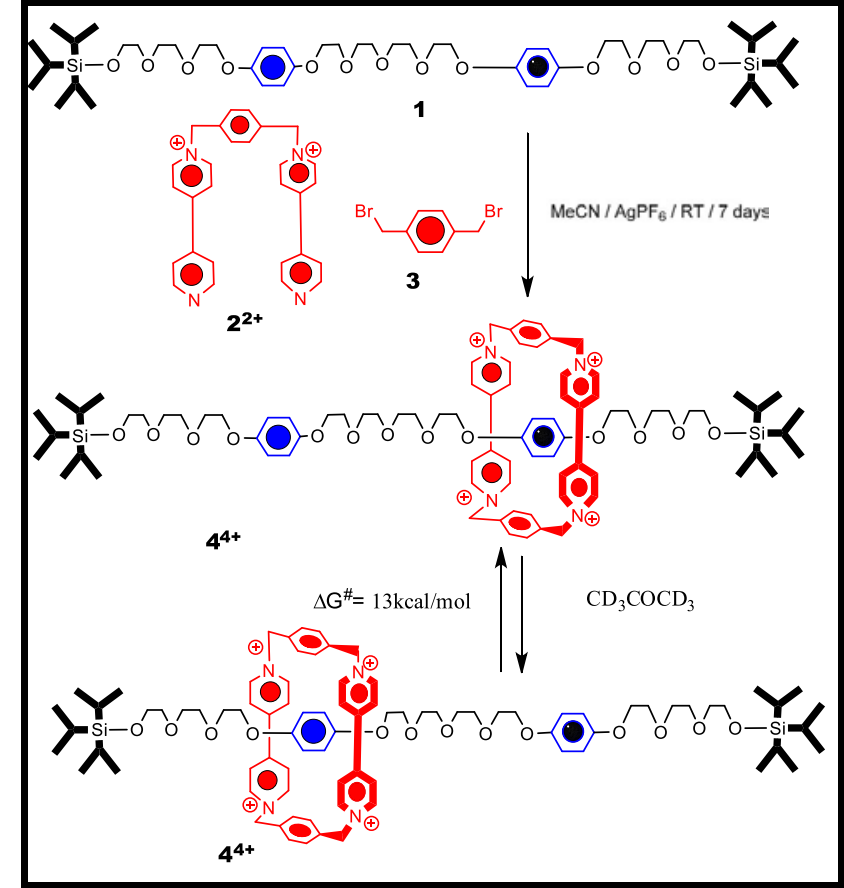

Figure 6: Scheme 1: Templated synthesis of the [2] rotaxane cyclo-bis (paraquat-pphenylene) cyclophane $\left(4^{4+}\right)$ and the shuttling process between the two degenerate recognition sites

\section{Application of Artificial Molecular Machines}

(I) Rotaxane-based Molecular Switch: The rotaxanes were found to exhibit translational isomerism in 1991 by Sir James Fraser Stoddart at the University of Sheffield, UK. They synthesised the $\pi$-electron-deficient cyclo-bis (paraquat- $p$ phenylene) cyclophane $4^{4+}$ as shown in Scheme 1 (Figure 6). Here the tetra-cationic cyclophane ring moves between two degenerate hydroquinone recognition sites. The non-covalent interactions that enable the synthesis of $4^{4+}$ are the interactions with the $\pi$-electron rich guest by $\pi-\pi$ stacking. It was prepared by clipping the $2^{2+}$ and 3 around 1 . The product found in $32 \%$ yield was observed to move between the two stations in the dumb-bell shaped component at 500 times per second at room temperature and $\mathrm{CD}_{3} \mathrm{COCD}_{3}$ and the energy barrier for this shuttling was found to be $13.0 \mathrm{kcal} \mathrm{mol}^{-1}$ (Anelli, Spencer \& Stoddart, 1991).

However, it cannot function as a switch since both the stations at 1 are similar, thereby possessing similar affinity for cyclophane. The first molecular switch ever reported had structural similarity with $4^{4+}$ and that was formed by introducing asymmetry at 
one of the two stations. One of the two 4,4'-biphenol residues were replaced by benzidine to introduce the asymmetry as shown in Scheme 2 (refer to figure 7). This slight structural change resulted in the molecule showing translational isomerism where the cyclophane has greater affinity to reside at the benzidine site because of its richness in $\pi$-electron that resulted in a greater $\pi$ - $\pi$ stacking interaction between the bipyridinium units of the cyclophane and benzidine sites.

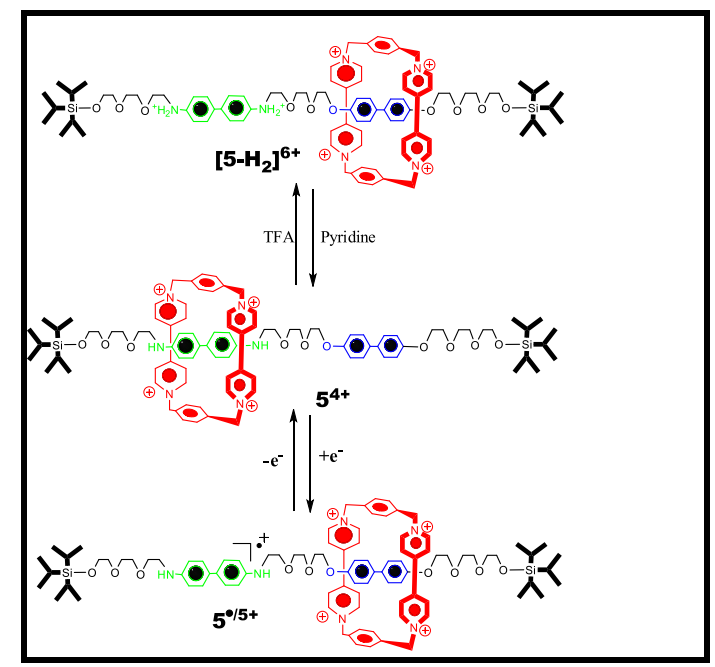

Figure 7: Scheme 2: Chemical and Electrochemical Switching [2] Rotaxane $5^{4+}$

Electrochemical Switching: As mentioned above, the cyclophane preferably resides at the benzidine station of the dumb-bell component. So, the benzidine site undergoes electrochemical oxidation to form $5^{5+}$. It is noteworthy that the tetra-cationic cyclophane initially present at the benzidine site makes the oxidation of benzidine more difficult. This insists the cyclophane to shift away towards the bi-phenol unit. However, the reaction is reversible as the benzidine can be reduced back to $5^{4+}$ again from $5^{5+}$ (Johnston, Warrener \& Gunter, 1998).

Chemical Switching: The $5^{4}+$ system is also stabilised by $\mathrm{C}-\mathrm{H}$...... $\mathrm{O}$ interactions between the bipyridinium hydrogen atoms and the polyether oxygen atoms. In $\mathrm{CD}_{3} \mathrm{CN}$ at $229 \mathrm{~K}$, the $1 \mathrm{H}$ NMR spectrum gives two different signals for the two forms of the system and the ratio between the two co-conformations of this molecule is $84: 16$ with $5^{4+}$ having the higher proportion. Addition of deuterated trifluoroacetic acid $\left(\mathrm{CF}_{3} \mathrm{CO}_{2} \mathrm{D}\right)$ causes protonation at the basic nitrogen atoms of the benzidine unit, causing shifting of the macro-chain towards the bi-phenol unit structure and hence the ${ }^{1} \mathrm{NMR}$ spectrum shows only one signal indicating the structure $\left[5-\mathrm{H}_{2}\right]^{6+}$. The addition of pyridine here results in deprotonation to yield back $5^{4+}$ again.

(ii) Catenane based Molecular Switch: Here Catenane acts as a molecular switch in a similar fashion as Rotaxane does. It also has two different recognition sites at one of the two rings as shown in Scheme 3 (refer to figure 8).

Here, the catenane $6 \mathrm{H}^{5+}$ consists of a cyclophane having two bipyridinium rings and an ammonium recognition site $(\mathrm{AMH})$ and a crown ether ring. Initially, the bipyridinium ring is surrounded by the crown ether ring in both $6 \mathrm{H}^{5+}$ and $6^{4+}$ as determined by the electrochemical properties and absorption spectra, indicating no switching of ring on deprotonation at $\mathrm{AMH}$ unit. Electrochemical measurements

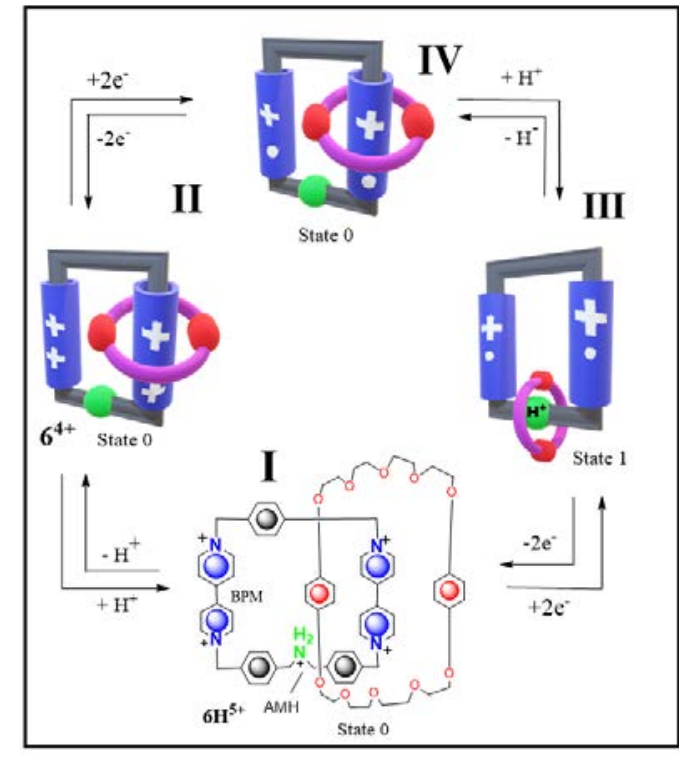

Figure 8: Scheme 3- Protonation of Catenane $6^{4+}$ and switching of the position of crown ether ring under acid base or redox inputs according to AND logic 
show when bipyridinium units of $6 \mathrm{H}^{5+}$ undergo one-electron reduction (electrochemical switching), the crown ether is displaced on AMH site as in III. Also, on deprotonation of III, the crown ether shifts to one of the bipyridinium unit. Thus, both reduction of bipyridinium units and protonation of $\mathrm{AMH}$ can bring the switching of the crown ether (Ghosh, Mermagen \& Schalley, 2002), like the AND logic because the results are dependent on two different inputs.

\section{CONCLUSION}

The recent development in the field of nanotechnology and molecular biology has opened a huge scope to explore and investigate more about different biochemical machinery and robots. We have concentrated in this review about the working of ATP synthase where the F0 rotates to generate the expected function that seems to be like a turbine used for generation of electricity or in nuclear power plants. This shows that nature has made this innovation and created this technology much before humans artificially developed it. Thus, even though it seems impossible to construct molecular devices that mimic the natural ones, getting a thorough concept and detailed understanding about the biochemical devices and mechanism can empower us to apply these mechanisms in the actual physical world and can make developments in mechanical and chemical engineering, physics, mathematics, and biomedical sciences. A careful approach on designing these molecules may help thusly in future in designing complex molecules that can help in turn to play out a few capacities at molecular level.

\section{ACKNOWLEDGEMENT}

The authors express their sincere thanks to the Department of Biotechnology, Government of India for the funding from DBT-STAR College grant, under which this review project was conducted. They are also grateful to the Principal and the DBT-STAR Coordinator, Surendranath College, for their support and encouragement in implementing this review project at the undergraduate level.

\section{REFERENCES}

Anelli, P. L., Spencer, N., \& Stoddart, J. F. (1991). A molecular shuttle. Journal of the American Chemical Society, 113(13), 5131-5133.

Balzani, V., Credi, A., \& Venturi, M. (2003). Molecular logic circuits. ChemPhysChem, 4(1), 49-59.

Balzani, V.V., Gomez-Lopez, M. \& Stoddart, J.F. (1998). Molecular machines. Accounts of Chemical Research, 31(7), 405-14.

Block, S. M. (2007). Kinesin motor mechanics: binding, stepping, tracking, gating, and limping. Biophysical journal, 92(9), 2986.

Browne, W. R., \& Feringa, B. L. (2010). Making molecular machines work. Nanoscience and Technology: A Collection of Reviews from Nature Journals, 79-89.

Citi, S., \& Kendrick-Jones, J. (1987). Regulation of non-muscle myosin structure and function. Bioessays, 7(4), 155-159.

Endow, S. A. (2003). Kinesin motors as molecular machines. Bioessays, 25(12), 1212-1219.

Feynman, R. P. (1960). The wonders that await a micro-microscope. Saturday Review, 43(2), 45-47.

Ghosh, P., Mermagen, O., \& Schalley, C. A. (2002). Novel template effect for the preparation of [2] rotaxanes with functionalised centre pieces. Chemical communications, (22), 2628-2629.

Gibbons, I. R., \& Rowe, A. J. (1965). Dynein: a protein with adenosine triphosphatase activity from cilia. Science, 149(3682), 424-426.

Heard, A. W., \& Goldup, S. M. (2020). Simplicity in the design, operation, and applications of mechanically interlocked molecular machines. ACS central science, 6(2), 117-128. 
Johnston, M., Warrener, R., \& Gunter, M. (1998). Templated formation of multi-porphyrin assemblies resembling a molecular universal joint. Chemical Communications, (24), 2739-2740.

Jones, P. C., \& Fillingame, R. H. (1998). Genetic Fusions of Subunit c in the F0Sector of H+-transporting ATP Synthase: FUNCTIONAL DIMERS AND TRIMERS AND DETERMINATION OF STOICHIOMETRY BY CROSS-LINKING ANALYSIS. Journal of Biological Chemistry, 273(45), 29701-29705.

Katchalsky, A. (2003). How protein motors convert chemical energy into mechanical work. Molecular motors.

Knowles, A. F., \& Penefsky, H. S. (1972). The subunit structure of beef heart mitochondrial adenosine triphosphatase: Physical and chemical properties of isolated subunits. Journal of Biological Chemistry, 247(20), 6624-6630.

Larrick, J. W., Larrick, J. W., \& Mendelsohn, A. R. (2018). ATP Synthase, a Target for Dementia and Aging? Rejuvenation research, 21(1), 61-66.

Le, N. Q. K., Yapp, E. K. Y., Ou, Y. Y., \& Yeh, H. Y. (2019). iMotor-CNN: Identifying molecular functions of cytoskeleton motor proteins using 2D convolutional neural network via Chou's 5-step rule. Analytical biochemistry, 575, 17-26.

Lehn, J. M. (1996). Supramolecular chemistry and chemical synthesis. In Chemical Synthesis (pp. 511524). Springer, Dordrecht.

Lipmann, F. (2013). Metabolic generation and utilization of phosphate bond energy. In A Source Book in Chemistry, 1900-1950 (pp. 381-383). Harvard University Press.

Rigler, R., Orrit, M., \& Basché, T. (Eds.). (2012). Single molecule spectroscopy: Nobel conference lectures (Vol. 67). Springer Science \& Business Media.

Sauvage, J. P. (1998). Transition metal-containing rotaxanes and catenanes in motion: toward molecular machines and motors. Accounts of chemical research, 31(10), 611-619.

Schill, G., \& Lüttringhaus, A. (1964). The preparation of catena compounds by directed synthesis. Angewandte Chemie International Edition in English, 3(8), 546-547.

Sielaff, H., Rennekamp, H., Engelbrecht, S., \& Junge, W. (2008). Functional halt positions of rotary FOF1-ATPase correlated with crystal structures. Biophysical Journal, 95(10), 4979-4987.

Wagner, P. D., \& Giniger, E. (1981). Hydrolysis of ATP and reversible binding to F-actin by myosin heavy chains free of all light chains. Nature, 292(5823), 560-562.

Wang, H., \& Oster, G. (1998). Energy transduction in the F 1 motor of ATP synthase. Nature, 396(6708), 279-282.

Weeds, A. G., \& Lowey, S. (1971). Substructure of the myosin molecule: II. The light chains of myosin. Journal of molecular biology, 61(3), 701-725. 


\title{
Variational Monte Carlo Approach for Solving Quantum Mechanical Systems
}

\author{
Rohit Kumar Thapa, Subha Pritam Pal, Soumyadeeep Mandal, Sourav Mitra* \\ Department of Physics, Surendra Nath College, Kolkata, India \\ ${ }^{*}$ Corresponding Author's Email: hisourav@gmail.com
}

\begin{abstract}
Complex quantum mechanical systems cannot be solved exactly using analytic methods and must be solved numerically. The variational Monte Carlo approach is one of such techniques to simulate physical systems numerically and is widely used to perform ab initio calculations on the system. In this chapter, the authors employ the variational Monte Carlo method to solve the time-independent Schrödinger equation for calculating the ground-state energy of harmonic and anharmonic oscillators which are of great interest in any quantum mechanical problems. The authors found that the results from their numerical analysis are in excellent agreement with the analytical results for known systems. Nonetheless, the numerical method presented here can also be applied to various other complex systems.
\end{abstract}

Keywords: Monte Carlo Method; Schrödinger Equation; Numerical Method; Harmonic and Anharmonic Oscillators

\section{INTRODUCTION}

The Schrödinger equation is one of the fundamental building blocks for determining the quantum nature of any particle. However, unfortunately this equation can be solved analytically only for very few simple quantum mechanical cases. For other systems, the behaviour of the quantum particle is somewhat more complex and thus the numerical techniques must be enforced to solve the equation and obtain its eigenvalues and eigenfunctions. One of such techniques is to use the Monte Carlo method to solve the Schrödinger equation numerically. Monte Carlo simulation methods are quite useful to simulate complex systems with many coupled degrees of freedom. Furthermore, use of Monte Carlo is preferable for evaluating high dimensional integrals. Monte Carlo can also be used to simulate equations that are difficult to solve by standard analytical and other numerical techniques. The authors shall explain two algorithms for solving the Schrödinger equation based on the variational principle for the ground-state energy of a quantum mechanical simple harmonic and anharmonic oscillator along with a detailed comparison between those two methods. In the literature review section, the authors review the basic variational method, generation of pseudorandom numbers, random walks, and metropolis sampling. Then they study two methods based on the variational Monte Carlo techniques and their applications to two-dimensional problems - harmonic and anharmonic oscillator after that conclusion has been provided.

\section{LITERATURE REVIEW}

In quantum mechanics, the variational method, which is based on the variational principle, is one way of finding approximations to the lowest energy eigenstate or ground state, and some excited states (Hammond, Lester \& Reynolds, 1994; Gould, Tobochnik \& Christian, 1988). The method involves choosing a trial wave function depending on one or more parameters (called variational parameters) and finding the best possible values of those parameters for which the expectation value of the energy state is the lowest. The expectation value of the energy eigenstate obtained in this way then gives an upper bound of the ground state energy. Similarly, the ground state wavefunction can be approximated by fixing those variational parameters to their best possible values.

\section{Theory}

If a good approximation to the ground state energy for a system described by the Hamiltonian $\mathrm{H}$ needs 
to be calculated for which the (time-independent) Schrödinger equation cannot be solved exactly. For such a system one can employ the variational method for solving the Schrödinger equation to put a bound on the ground state energy (Hammond, Lester \& Reynolds,1994). According to the variational principle, the expectation value of the energy of a trial wave function $\psi$ given by,

$$
E[\psi]=\frac{\int \psi^{*} H \psi d x}{\int \psi^{*} \psi d x}
$$

will be a minimum for the exact ground state wave function. The functional $E[\psi]$ thus provides an upper bound to the ground state energy. A form for $\psi$ is chosen that contains a set of variational parameters, $\alpha \equiv \alpha_{1}, \alpha_{2}, \ldots, \alpha_{n}$. The functional form of $\psi$ and these variational parameters will determine the ultimate accuracy of the method. They then minimize the $E[\psi]$ with respect to the variation of its parameters to find the best choice of $\psi$. This will also provide an upper bound to the true energy.

\section{Introducing the Monte Carlo Methods}

Monte Carlo methods are a sophisticated class of computational algorithms which is widely used in simulating physical and mathematical systems. Because of their reliance on repeated computation of random or pseudorandom numbers, these methods are most suited to calculation by a computer and tend to be used when it is unfeasible or impossible to compute an exact result with a deterministic algorithm. These methods are particularly useful in studying systems with many coupled degrees of freedom. Furthermore, use of Monte Carlo is advantageous for evaluating higher dimensional integrals which can be used to solve several complex classes of equations that are somewhat impossible to solve by other standard numerical techniques. Here the authors are only interested in various aspects of statistics and simulation of the Monte Carlo solution of the Schrödinger equation. They begin with a discussion of generation of pseudorandom numbers and then present the essentials of Monte Carlo sampling and simulation. For details regarding the use of Monte Carlo methods in numerical analysis, they refer the readers to see Rubinstein and Kroese (2016); Press et al. (1992).

\section{MATERIALS AND METHODS}

Variational Quantum Monte Carlo Methods

In this project, the authors are only interested in solving simple one-dimensional quantum problems using variational methods. They have used two Monte Carlo methods for implementing the variational method (Bolton, 1996; Pottorf et al. 1999).

\section{Method 1}

Consider a one-dimensional physical system whose Hamiltonian operator $H=T+V$, where $T=$ $-\frac{h^{2}}{2 m} \frac{d^{2} \psi(x)}{d x^{2}}$ is the kinetic energy and $\mathrm{V}=\mathrm{V}(\mathrm{x})$ is the potential energy of the system. The variational principle states that for an arbitrary trial wave function $\psi(x)$.

$$
\langle H\rangle=\frac{\int \psi^{*}(x) H \psi(x) d x}{\int \psi^{*}(x) \psi(x) d x} \geq E_{0}
$$

where $E_{0}$ is the ground state energy eigenvalue of the system. They can base the variational approach on the expansion of $\psi$ in terms of a complete set of basis functions $\phi_{n}$

$$
\psi=\sum_{n} c_{n} \phi_{n}
$$

Their first Monte Carlo approach is to retain a finite number of terms in this equation and to vary the unknown coefficients ${ }_{n}$, until a reasonable estimate of the minimum $\langle$ of $H\rangle$ is found. To implement this procedure, they need to calculate the matrix elements

$$
\begin{aligned}
& T_{n m}=\left\langle\phi_{n}|T| \phi_{m}\right\rangle \\
& V_{n m}=\left\langle\phi_{n}|V| \phi_{m}\right\rangle
\end{aligned}
$$


Once the matrix elements are known, the expectation value $\langle H\rangle$ is given by

$$
\langle H\rangle=\frac{\sum_{n, m} c_{n}^{*} c_{m}\left(T_{n m}+V_{n m}\right)}{\sum_{n}\left|c_{n}\right|^{2}}
$$

A Monte Carlo approach in two one-dimensional cases - simple harmonic oscillator (as a test of the method) and anharmonic oscillator were applied.

Harmonic Oscillator: Here the Variational Monte Carlo method were used to solve for the ground state energy of a particle in a harmonic potential. The standard time independent Schrödinger equation

$$
H \Psi=E \Psi
$$

with the Hamiltonian $H=-\frac{h^{2}}{2 m} \frac{d^{2} \psi(x)}{d x^{2}}+V(x)$ and potential $V(x)=\frac{1}{2} m \omega^{2} x^{2}$. Here it is obvious to choose the basis set to be the solution of harmonic oscillator problem

$$
\phi_{n}=\left(\frac{m \omega}{\pi \hbar}\right)^{1 / 4} \frac{e^{-\frac{x^{2}}{2}} H_{n}(x)}{\sqrt{2^{n} n !}}
$$

where the Hermite polynomials $H_{n}(x)$ have the form

$$
H_{n}(x)=(-1)^{n} e^{x^{2}} \frac{d^{n}}{d x^{n}} e^{-x^{2}}
$$

Now in the units of $m=\omega=\hbar=1$, our $T=-\frac{1}{2} \frac{d^{2} \Psi(x)}{d x^{2}}$ and $V=\frac{1}{2} x^{2}$. The matrix elements $T_{n m}$ and $V_{n m}$ can be evaluated analytically for this choice of basis set. The results are

$$
\begin{aligned}
T_{n m} & =\frac{1}{2}\left(n+\frac{1}{2}\right) & \text { if } n=m \\
= & \frac{1}{4} \sqrt{(n+1)(n+2)} & \text { if } n=m+2 \\
= & 0 & \text { otherwise }
\end{aligned}
$$

and $V_{n m}=T_{n m}$ for a harmonic oscillator. The researchers expand $\psi$ in terms of a complete set of this basis functions $\phi_{n}$. They take the initial guess $c_{0}=1$ and $c_{n}=0$ for $n \neq 0$ and retain the first few terms in the equation. Then they vary $c_{n}$ by a random amount between $[-\delta, \delta]$, compute the corresponding change in energy $\Delta E$ and accept the change only if $\Delta E<0$. They repeat these steps until they get their best estimate for the ground state energy $E_{0}$. For harmonic oscillator $E$ will never decrease ifthey start from this choice, becausethey know this is exact for harmonic oscillators. In fact, they can get the same E0 for different choices of initial conditions.

Anharmonic Oscillator: This method can also be used for solving the one-dimensional anharmonic oscillator problem where the potential energy is (in their units) $V(x)=\frac{1}{2} x^{2}+t x^{4}$, where $t$ is the anharmonicity term. It is reasonable to choose the basis set to be the normalized stationary states of the harmonic oscillator. The matrix elements ${ }_{n m}$ and $V_{n m}$ can be evaluated analytically for this case also. $T_{n m}$ are the same as before, but $V_{n m}$ are more complicated. They follow the same procedure as they did for the harmonic oscillator and get the estimated ground state energy.

\section{Method 2}

The Schrödinger equation can be solved numerically by a Monte Carlo method using a variational 
wave function. In this methodthey start from an initial probability distribution and calculate the exact minimum energy of the quantum particle by varying the trial wavefunction. This minimum energy state as a function of the variational parameters is basically the system's eigenstate i.e, the ground state. This method ensures that the minimum in the standard deviation will correspond to the minimum of the energy. For details of this method, the researchers refer the readers to see Pottorf et al. (1999).

They begin with the standard wave equation

$$
H \Psi=E \Psi
$$

with the Hamiltonian $H$ and $E$ is the energy eigenvalue corresponding to an eigenstate $\psi$ of the problem. The researchers will show how a Monte Carlo scheme can be implemented here to estimate the approximated ground state energy for a given trial wavefunction $\Psi(\alpha)$. For that, lets first define a local energy, $E_{L}$, as

$$
E_{L}=\frac{H \Psi}{\Psi}
$$

For a set of $\alpha$, next they define the following energy

$$
\left\langle E_{L}\right\rangle_{\Psi^{2}}=\frac{\int_{-\infty}^{\infty} \Psi(x) H \psi(x) d x}{\int_{-\infty}^{\infty} \psi^{2}(x) d x}=\frac{\int_{-\infty}^{\infty} \Psi^{2}(x) E_{L}(x) d x}{\int_{-\infty}^{\infty} \psi^{2}(x) d x}
$$

where $x$ represents random positions in one dimension. To estimate the energy, they sample the random numbers from the probability density

$$
P(x)=\frac{\Psi^{2}(x)}{\int_{-\infty}^{\infty} \psi^{2}(x) d x}
$$

Suppose that a single walker visits the points $x^{0}, x^{1}, \ldots, x^{m}$ during the walk, also consider an ensemble of walkers $x=x_{1}, x_{2}, \ldots, x_{N}$, each performing independent random walks. They use Metropolis algorithm to reach a distribution $\Psi^{2}(x)$ and the walkers are distributed according to $\Psi^{2}$ . The random numbers, $x_{j}^{i}$, obtained are used to calculate the Monte Carlo average energy

$$
\left\langle E_{L}\right\rangle_{\Psi^{2}}=\lim _{N \rightarrow \infty} \lim _{m \rightarrow \infty} \sum_{j=1}^{N} \sum_{i=1}^{m} E_{L}\left(x_{j}^{i}\right)
$$

where $m$ is the ensemble size of random numbers $\left\{x^{1}, \ldots, x^{i}, \ldots, x^{m}\right\}$ and $\mathrm{N}$ is the total number of ensembles i.e., the number of Monte Carlo steps used here?Note that, the generated ensembles must follow the underlying probability distribution $P(x)$ function to properly evaluate $\left\langle E_{L}\right\rangle$. For that, they choose a given ensemble by the Metropolis method that uses an acceptance and rejection process of random numbers closely obeying the distribution like $\Psi^{2}(x)$. Afterthey generate ensemble of random numbers, the acceptance criterion is imposed in such a way that the probability $(A)$ of moving from an initial random number of the ensemble, ${ }_{i}$, to a new random number, $x_{k}$,

$$
A=\frac{\Psi^{2}\left(x_{k}\right)}{\Psi^{2}\left(x_{i}\right)}
$$

is restricted with a maximum value of 1 . The idea is for any two $\Psi^{2}\left(x_{k}\right)$ and $\Psi^{2}\left(x_{i}\right)$, they will accept the move from $i \rightarrow k$ with acceptance ratio $A$ equal to 1 if $\Psi^{2}\left(x_{k}\right) \leq \Psi^{2}\left(x_{i}\right)$, and also they will accept the move for $\Psi^{2}\left(x_{k}\right)<\Psi^{2}\left(x_{i}\right)$, but with the acceptance ratio or probability equal to $\Psi^{2}\left(x_{k}\right) / \Psi^{2}\left(x_{i}\right)$. Thus, the acceptance probability can be written as 


$$
A=\min \left(\frac{\Psi^{2}\left(x_{k}\right)}{\Psi^{2}\left(x_{i}\right)}, 1\right)
$$

They choose a wider sampling range to ensure a broader ensemble limit. The same process is replicated for each member of an ensemble. Hence, it can be expected that, the accepted ensembles used to evaluate the Monte Carlo algorithm for the average energy will provide a very good approximation to the exact ground state of the system, providedthey use sufficiently large limits of $\mathrm{m}$ and $\mathrm{N}$.

In the process of evaluating the energy, it is equally useful to estimate the corresponding standard deviation, defined as

$$
\sigma(E[\psi])=\sqrt{\frac{\left\langle E_{L}^{2}\right\rangle_{\psi^{2}}-\left\langle E_{L}\right\rangle_{\psi^{2}}^{2}}{m N-1}} .
$$

They know that the standard deviation of the local energy will be zero for an exact trial wave function. So, in the Monte Carlo method, minimum in the standard deviation corresponds to the minimum of $\left\langle E_{l}\right\rangle_{\psi^{2}}$, and this is the main thing that they aim to find out through this analysis. They again apply this approach for two different scenarios described below.

Harmonic Oscillator: For the harmonic oscillator, they begin by assuming a trial wave function of the form

$$
\Psi(x)=\exp \left(-\alpha x^{2}\right)
$$

Using this wavefunction the local energy becomes, in units of $\hbar \omega$,

$$
E_{L}=\alpha+\left(\frac{1}{2}-2 \alpha^{2}\right) x^{2}
$$

For a set of $\alpha$, they have calculated $\left\langle E_{L}\right\rangle_{\psi^{2}}$ and $\sigma(E[\psi])$. Since $\left\langle E_{L}\right\rangle_{\psi^{2}}$ would be exact if an exact trial wave function is used i.e., the standard deviation in the local energy will become zero.

Anharmonic Oscillator: This method can also be used for solving the one-dimensional anharmonic oscillator problem where the potential energy is (in our units) $V(x)=\frac{1}{2} x^{2}+t x^{4}$. They take the trial wave function as

$$
\Psi(x)=\exp \left(-\alpha x^{2}-\beta x^{4}\right)
$$

where $\alpha$ and $\beta$ are the variational parameters. This choice of the trial wave function is justified by the result for anharmonic oscillator by method 1 . So, the local energy becomes

$$
E_{L}=-\frac{1}{2}\left[-\left(2 \alpha+12 \beta \boldsymbol{x}^{2}\right)+\left(2 \alpha \boldsymbol{x}+\mathbf{4} \boldsymbol{\beta} \boldsymbol{x}^{3}\right)^{2}\right]+\frac{1}{2} \boldsymbol{x}^{2}+b \boldsymbol{x}^{4}
$$

They follow the same procedure asthey did for the harmonic oscillator and get the estimated ground state energy of anharmonic oscillator.

\section{RESULT AND DISCUSSION}

\section{Method 1}

For both harmonic and anharmonic oscillator they have taken the initial guess $c \_0=1$ and $c \_n=0$ for $n \neq 0$. They have studied the results for various cases - by taking first 5 terms and first 10 terms and varying our $\delta$. They have taken our Monte Carlo steps to be very large $(=10000)$. For anharmonic oscillator they have taken different anharmonicity terms $b$ and obtained corresponding ground- state energies $E_{-} 0$ (refer to table 1). 
Table 1: Results from the Current Analysis Using Method 1 for Harmonic and Anharmonic Oscillators

\begin{tabular}{|l|c|c|c|}
\hline Case & $\mathrm{n}$ & $\mathrm{t}$ & $\mathrm{E}_{0}$ \\
\hline \multirow{3}{*}{ Harmonic } & 5 & 0.0 & 0.5000000 \\
\cline { 2 - 4 } & 10 & 0.0 & 0.5000000 \\
\hline Anharmonic & 5 & 0.1 & 0.5604791 \\
\cline { 2 - 4 } & & 0.01 & 0.5074735 \\
\cline { 2 - 4 } & \multirow{2}{*}{10} & 0.1 & 0.5750000 \\
\cline { 3 - 4 } & & 0.01 & 0.5075000 \\
\hline
\end{tabular}

Next for anharmonic oscillator, they have taken the coefficients cn (up to $n=5$ ) corresponding to the ground-state energy and calculated the ground-state wave function (refer to table 2). Then they have plotted and tried to fit it by a function $f(x)=a \exp \left(-\alpha x^{\wedge} 2-\beta x^{\wedge} 4\right)$ for different $t$ (Figure 1 and 2 ).

Table 2: Best-fit parameter values from Method 1 for the ground-state wave function of anharmonic oscillator with different anharmonic coefficients $t=0.1$ and 0.01

\begin{tabular}{|l|c|c|c|c|}
\hline Value of $t$ & $a$ & $\alpha$ & $\beta$ & $E_{0}$ \\
\hline 0.1 & 0.752089 & 0.579510 & 0.0136222 & 0.5604791 \\
\hline 0.01 & 0.737127 & 0.514504 & 0.0026657 & 0.5074735 \\
\hline
\end{tabular}

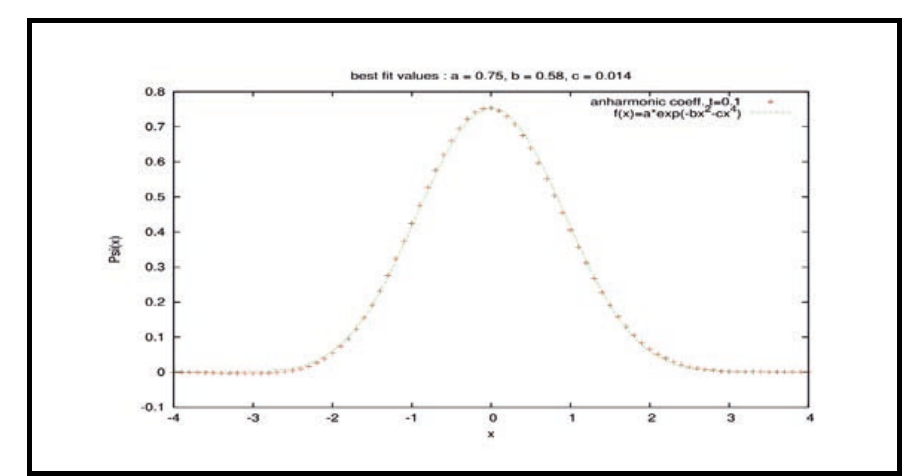

Figure 1: Plot for the ground-state wave function (red dots) of anharmonic oscillator with $t=0.1$. The green line represents corresponding fit with the fitting function $f(x)=a \exp \left(-\alpha x^{\wedge} 2-\beta x^{\wedge} 4\right)$

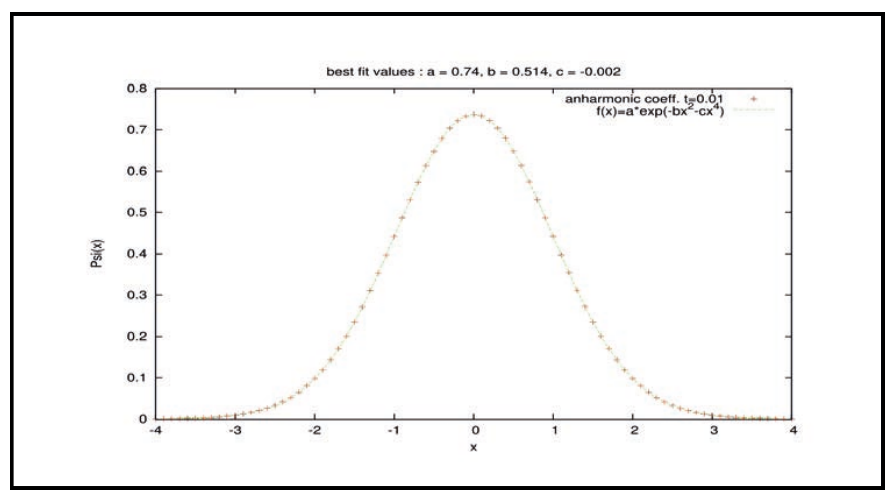

Figure 2: Plot for the ground-state wave function (red dots) of anharmonic oscillator with $t=0.01$. The green line represents corresponding fit with the fitting function $f(x)=a \exp \left(-\alpha x^{\wedge} 2-\beta x^{\wedge} 4\right)$ 


\section{Method 2}

The Monte Carlo process as described in this study has been employed for one dimensional simple harmonic oscillator and anharmonic oscillator. They have first found the no. of steps for equilibration i.e., no. of steps necessary to bring the system in equilibrium. They obtained that to get a reasonable estimate for the ground state energy, it should be enough to take the ensemble size $m=1000$ and the Monte Carlo steps N=10000 for these cases. See Figure 3 below.

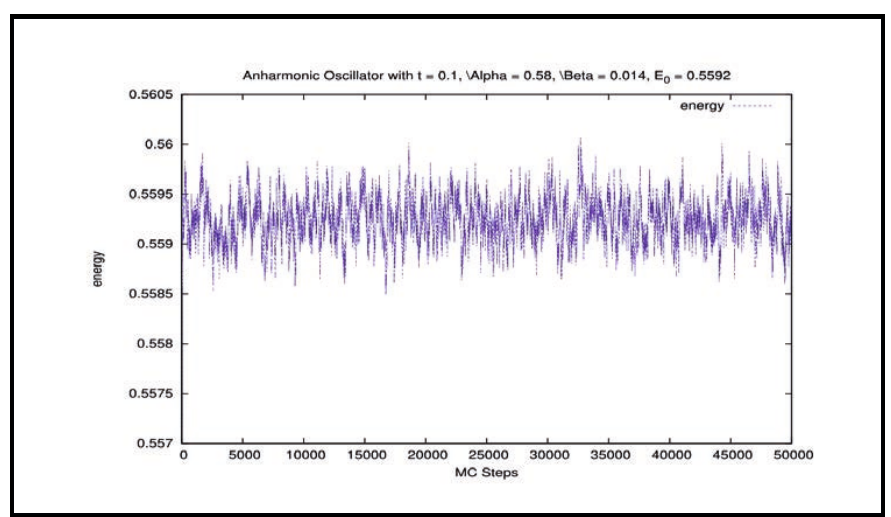

Figure 3: Energy vs. MC step plot for anharmonic oscillator (with $t=0.1$ ) to show the equilibration. Here $E 0 \approx 0.56$ and $\alpha=0.58$ and $\beta=0.014$

For harmonic oscillator they have taken trial wave function $\Psi(x)=a \exp \left(-\alpha x^{\wedge} 2\right)$. They varied $\alpha$ and got the minimum energy (and simultaneously minimum $\sigma$ ) for $\alpha=0.5$. See Figure 4 and Figure 5 below.

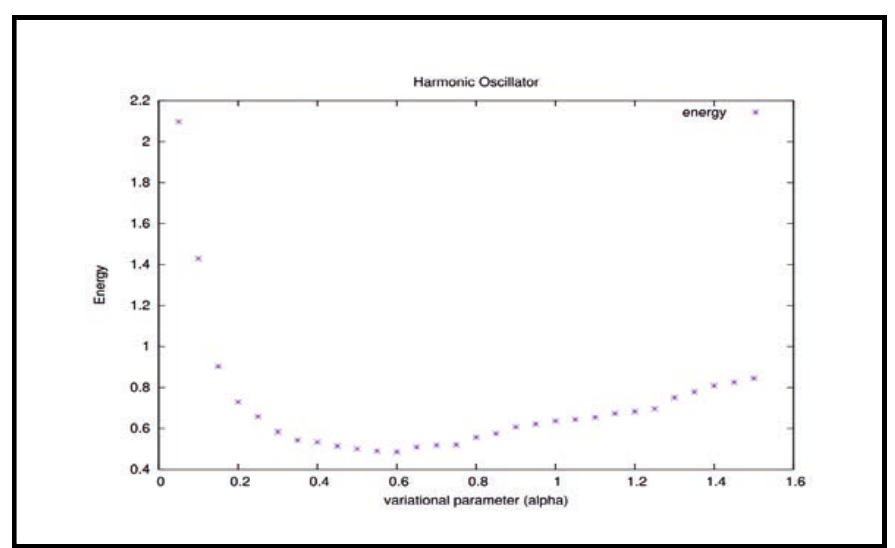

Figure 4: Energy of harmonic oscillator for different a

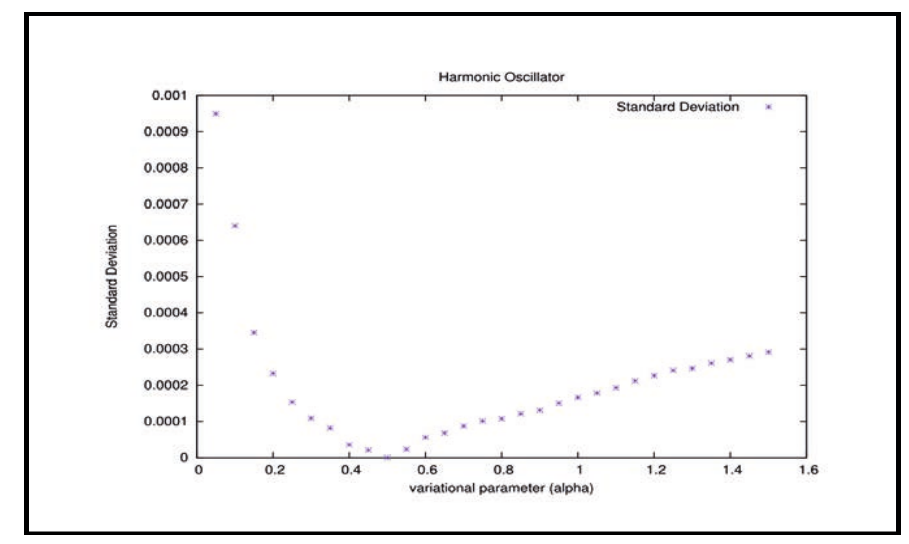

Figure 5: Standard deviation for different a

For anharmonic oscillator they have taken our wave function as $\Psi(x)=a \exp \left(-\alpha x^{\wedge} 2-\beta x^{\wedge} 4\right)$ motivated from 
the result of method 1 for anharmonic oscillator). Here they have varied both $\alpha$ and $\beta$ (over a range $0 \leq \alpha \leq$ 1 and $0 \leq \beta \leq 0.1$ ) to get minimum energy as well as minimum $\sigma$. The results are summarized in the tables $3 \& 4$ below:

Table 3: Results from the current analysis using Method 2 for anharmonic oscillator with different values of $\alpha$

\begin{tabular}{|c|c|c|}
\hline$\alpha$ & $E_{\min }$ & $\sigma_{\mathrm{Emin}}$ \\
\hline 0.25 & 0.6580016 & $1.5313852 \mathrm{E}-04$ \\
\hline 0.50 & 0.5000000 & $0.0000000 \mathrm{E}+00$ \\
\hline 0.75 & 0.5202308 & $1.0085671 \mathrm{E}-04$ \\
\hline 1.00 & 0.6361769 & $1.6603740 \mathrm{E}-04$ \\
\hline
\end{tabular}

Table 4: Same as Table 3 but with different combinations of aand Bfor different anharmonic coefficients $t=0.1$ and 0.01

\begin{tabular}{|l|c|c|c|c|}
\hline Value of $\mathrm{t}$ & $\alpha$ & $\beta$ & $\mathrm{E}_{\min }$ & $\sigma_{\mathrm{Emin}}$ \\
\hline 0.1 & 0.58 & 0.014 & 0.5592714 & $5.7481116 \mathrm{E}-06$ \\
\hline 0.01 & 0.52 & 0.002 & 0.5082759 & $1.0724962 \mathrm{E}-06$ \\
\hline
\end{tabular}

\section{CONCLUSION}

This work presents two variational Monte Carlo methods that can be used to obtain the ground-state energies of a one-dimensional harmonic and anharmonic oscillator. In fact, they can use the second method (here known as method 2) for two-and three-dimensional cases also. Our key findings from this analysis are that the results from the numerical techniques used here are in excellent agreement with the exact analytical ones. It is expected that for systems where the exact wave function is unknown, the minimum energy can still correspond to a minimum in the standard deviation which can be obtained using method 2. In future, they hope to apply these two methods to obtain ground state energies of more complex quantum systems. For such cases, it might happen that the number of variational parameters would be more than two, then they have to use the Conjugate gradient method for this optimization and that could be the next important extension to the work done here.

\section{REFERENCES}

Bolton, F. (1996). Fixed-phase quantum Monte Carlo method applied to interacting electrons in a quantum dot. Physical Review B, 54(7), 4780.

Gould, H., Tobochnik, J., \& Christian, W. (2007). An introduction to computer simulation methods. Comput. Phys, 10, 652-653.

Hammond, B. L., Lester, W. A., \& Reynolds, P. J. (1994). Monte Carlo methods in ab initio quantum chemistry (Vol. 1). World Scientific.

Pottorf, S., Pudzer, A., Chou, M. Y., \& Hasbun, J. E. (1999). The simple harmonic oscillator ground state using a variational Monte Carlo method. European Journal of Physics, 20(3), 205.

Press, W. H., Teukolsky, S. A., Flannery, B. P., \& Vetterling, W. T. (1992). Numerical recipes in Fortran 77: volume 1, volume 1 of Fortran numerical recipes: the art of scientific computing. Cambridge university press.

Rubinstein, R. Y., \& Kroese, D. P. (2016). Simulation and the Monte Carlo method (Vol. 10). John Wiley \& Sons. 


\title{
A Review on the Phenomenological Theory of Phase Transitions
}

\author{
Argha Deb, Animesh Mallick, Avijit Sen Majumder, Deepanwita Das, Eram Tanwir, Asok Kumar \\ Das* \\ Department of Physics, Surendranath College, Kolkata, India \\ ${ }^{*}$ Corresponding Author's Email: haripurus@gmail.com
}

\begin{abstract}
In this brief review, the authors went through a phenomenological approach to describing phase transitions in general. The behaviour of apparently quite different systems undergoing a phase transition can be explained based on Landau's free energy theory which is reviewed in some detail here. The notion of a critical point and that of critical exponents is explored.
\end{abstract}

Keywords: Thermodynamics; Phase transitions; Order Parameter; Critical Phenomenon

\section{INTRODUCTION}

Phase transitions are ubiquitous around us. From water boiling in a saucepan kept on a stove to cosmological phase transitions in the early universe, phase transitions play a very important role in the world around us. A common theme in all phase transitions is some change in certain properties of the system (Zemansky \& Dittman, 1998; Goldenfeld, 2018). In the words of Landau, a phase transition is essentially associated with change in the symmetry properties of the system undergoing phase transition. For example, when a solid melts into the corresponding liquid phase, the translation symmetry associated with the solid crystal is completely lost because the liquid is essentially isotropic in its spatial distribution of particles. In this sense, a face-centered cubic crystal transforming into a bodycentered cubic crystal may also be characterized as a phase transition because it involves a change in the symmetry properties of the system even though superficially, they may both be classified as solids. This idea of recognizing the change in symmetry properties of a system and associating that with a phase transition has led to profound implications.

A little before the mid-20th century, it was observed that the description of a magnet undergoing a paramagnetic to ferromagnetic phase transition and a gas undergoing a liquefaction are not very different. In other words, although apparently a magnet appears to be quite different from a fluid, it shows a very similar behaviour to a fluid as far as the phase transition was concerned. This was a eureka discovery which led people to believe that phase transitions had some universal characteristics which did not depend on the details of the system. It was primarily this observation which led one of the greatest minds of all time, Lev Landau to formulate a phenomenological theory of phase transitions which eventually became his Nobel Prize winning discovery (Goldenfeld, 2018).

\section{LITERATURE REVIEW}

In this brief article, the authors reviewed some preliminary aspects of phase transitions from a rather phenomenological approach. The article is organized as follows. In this next section, the authors briefly reviewed the essential aspects of the liquid-gas phase transition taking the prototypical example of the van der Waals fluid. The notion of a critical point and critical exponents are discussed. Following that, in this manuscript the authors considered a prototypical model namely, the Ising model exhibiting a magnetic phase transition and approximately solve it using a mean field approach. It is then shown that the generic characteristics of such a phase transition exact coincide with that of the van der Waals fluid. Finally, the authors introduced the phenomenological theory of phase transitions due to Landau and show how phase transitions and their associated characteristics can be described in a general manner. Finally, the authors ended with some remarks on the limitations of Landau's theory. 


\section{Liquid-Gas Phase Transition}

The liquid-gas phase transition is something most of us a familiar with from our observations in daily life. The classic experiments due to Andrew on liquefaction of $\mathrm{CO}_{2}$ aroused the interest of physicists and chemists alike on the liquid-gas phase transition. It is well known that although the simplest equation of state describing a hydrostatic, i.e., $(P, V, T)$-system is the ideal gas equation of state, it is incapable of exhibiting any phase transition. Henceforth, several equations of state for gases have been proposed in the literature to explain the experimental observations on real gases. In what follows, the authors will consider the simplest, yet best known real gas model, namely the van der Waals' model whose equation of state reads,

$$
P=\frac{k_{B} T}{v-b}-\frac{a}{v^{2}}
$$

Where, $\mathrm{v}=\mathrm{V} / \mathrm{N}$ is the specific volume or the volume occupied by each molecule. All other symbols have their usual meanings from thermodynamics textbooks. The constant parameters $a$ and $b$ are both positive. While the former characterizes the strength of the intermolecular van der Waals interactions, the latter is the co-volume parameter which associates a finite volume to the fluid molecules.

According to the Andrew's experiments, there exists a critical temperature Tc below which one can observe the liquid phase on the $\mathrm{P}-\mathrm{v}$ indicator diagram. The isotherm at $\mathrm{T}=\mathrm{Tc}$ is termed as the critical isotherm and it is therefore clear that it must satisfy,

$$
\left(\frac{\partial P}{\partial v}\right)=0=\left(\frac{\partial^{2} P}{\partial v^{2}}\right)
$$

with the latter condition arising due to the fact that the critical point occurs as an inflexion point. For the van der Waals fluid, these conditions give,

$$
P_{c}=\frac{a}{27 b^{2}}, \quad v_{c}=3 b, \quad T_{c}=\frac{8 a}{27 k_{B} b}
$$

Interestingly, one has

$$
\frac{k_{B} T_{c}}{P_{c} v_{c}}=\frac{8}{3}
$$

independent of any other parameters. These values of the pressure, (specific) volume and temperature define a very special point on the space of thermodynamic states which is termed as the critical point. It is this point at which the liquid and the gaseous phases become thermodynamically indistinguishable. This can be explained as follows:

The (P,T)-phase diagram of a generic fluid is well known (Zemansky \& Dittman, 1998). Ignoring the lines associated with the solid phase, there is a line separating the liquid and gaseous phases. For values of pressure and temperature to the left of this line, one finds the liquid phase whereas for values to the right, one finds the gaseous phase. In this sense, this line separates the liquid and gaseous phases such that there is a discontinuous jump in the specific volume $v$ as one cross this line and goes from one phase to another. Such a line is called the line of first order transition and there is always a jump in the specific volume, i.e., $v_{\text {gas }}-v_{\text {liquid }}>0$ as one crosses it. However, this line of first order transition terminates at a very special point at which $\mathrm{v}_{\text {gas }}-\mathrm{v}_{\text {liquid }}=0$. This is precisely the critical point described by the critical values of pressure, volume, and temperature. It is that point at which the distinction between the liquid and the gaseous phases vanishes.

It is interesting to note that with a few mathematical manipulations of the van der Waals' equation of state, one can show that in the vicinity of the critical point, quantities such as the specific heat $\mathrm{C}_{\mathrm{v}}$, the isothermal compressibility $\mathrm{k}_{\mathrm{T}}$, etc show an interesting power law behaviour which can be summarized as,

$$
C_{v}=\frac{3 k_{B}}{2} \sim|1-\tau|^{0}, \quad \kappa_{T} \sim|1-\tau|^{-1}, \quad p-1 \sim(\vartheta-1)^{3}, \quad \vartheta_{\text {gas }}-\vartheta_{\text {liquid }} \sim \sqrt{|1-\tau|}
$$


where one defines the dimensionless variables,

$$
p=\frac{P}{P_{c}}, \quad \vartheta=\frac{v}{v_{c}}, \quad \tau=\frac{T}{T_{c}}
$$

In other words, $(p, \vartheta, T)=(1,1,1)$ is the critical point. Therefore, several physical parameters scale with each other near the critical point in a power law fashion with some exponents. These are famously known as the critical exponents. With this background, the authors shall next move to their next example, which is that of a magnetic system.

\section{Magnetic Phase Transitions}

In this section, the authors will consider magnetic phase transitions. For that purpose, they shall consider a simple prototypical model known as the Ising model (Goldenfeld, 2018). A typical Ising model is defined as a square lattice with a spin half particle sitting at each lattice site. Thus, lattice site can be either in an 'up' state or a 'down' state. The lattice shall be taken to be consistent of $\mathrm{N}$ such identical spins and can be extended in any number of spatial dimensions $d \geq 1$. The spins can interact with an external magnetic field $\mathrm{H}$ but they also do interact with each other. In the simplest case, each spin can interact with its nearest neighbours. In such a scenario, the Hamiltonian of the system is given as,

$$
\widetilde{H}=-J \sum_{\langle i j>} S_{i} S_{j}-H \sum_{i} S_{i}
$$

where $S_{i}= \pm 1$ signifying up/down states and $<i j>$ implies nearest neighbour interactions. The parameter $\mathrm{J}$ characterizes the coupling between the spins. If $\mathrm{J}=0$, the spins are non-interacting, and the system behaves like a paramagnet which does not have intermolecular interactions but can only interact with an external field. Now, if $\mathrm{J}>0$ the spins prefer to stay aligned with one another to minimize their energy (note the negative sign) and the interaction is said to be ferromagnetic. On the other hand, if $\mathrm{J}<0$, the neighbouring spins prefer to stay oppositely aligned and the interaction is said to be anti-ferromagnetic.

To determine the thermodynamic properties, one has to compute the partition function $Z$ from where one has the free energy as $F=-N k_{B} T \ln Z$. However, computing the partition function is not an easy task and exact computations can be performed for very limited cases. It is exactly solvable in the onedimensional case $(\mathrm{d}=1)$ where the partition function reads (in the limit that $\mathrm{N}$ is large),

$$
Z=\left[e^{\frac{J}{k_{B} T}} \cosh \left(\frac{H}{k_{B} T}\right)+\sqrt{e^{-\frac{2 J}{k_{B} T}}+e^{\frac{2 J}{k_{B} T}} \sinh ^{2}\left(\frac{H}{k_{B} T}\right)}\right]^{N}
$$

However, it is easy to check that for any non-zero temperature, i.e., $T>0$, it is impossible to have a ferromagnetic phase, i.e., non-zero magnetization with $\mathrm{H}=0$. In other words, if there is no externally applied field, i.e., then at any non-zero temperature, the model fails to exhibit spontaneous magnetization, which is a characteristic of the ferromagnetic phase.

However, this is not the case in higher dimensions where the Ising model exhibits the paramagneticferromagnetic phase transition. Since, it is extremely tedious to solve the Ising model even in two dimensions, one typically resorts to solving it approximately using a mean field theory. This approach assumes that each spin feels an average effect due to the other spins. This averaged or coarse-grained effect of interactions is what makes it possible to solve the Ising model approximately in this method. Employing mean field theory, the expression for magnetization reads the following,

$$
M=\tanh \frac{H+J q M}{k_{B} T}
$$

Where, $q$ is the coordination number, i.e., number of nearest neighbours of each spin. For example, in one dimension $q=2$ while in two dimensions $q=4$. If they now put $H=0$, one can have two situations: If $J q<k_{B} T$, the above equation admits only one solution which is $\mathrm{M}=0$. However, if $J q>k_{B} T$, then there are three solutions $M=0, \pm M_{0}$ (see figure 1 below). 
(a)

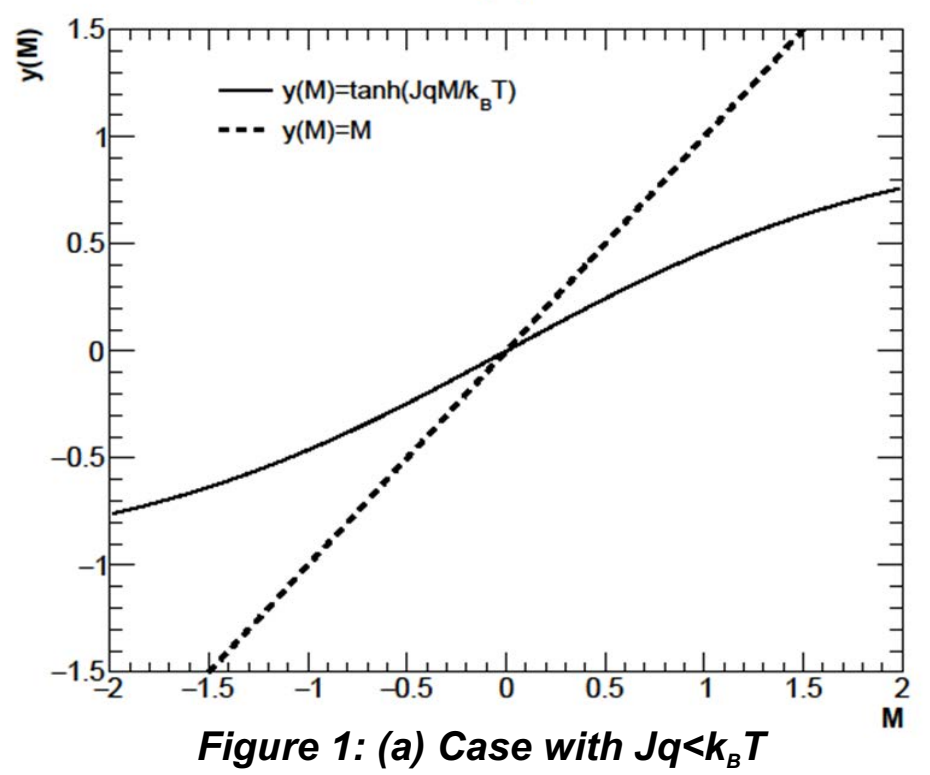

The temperature separating these two regimes is,

$$
T_{c}=\frac{J q}{k_{B}}
$$

and plays the role of a critical temperature. Below the critical temperature, i.e., $T<T$ c one can observe that the system can show spontaneous magnetization $M= \pm \mathrm{M}_{0}$. It can be checked that for this case the solution $M=0$, i.e., the non-magnetized solution is an unstable one because the free energy is not minimum there and a slight perturbation shall magnetize it. Thus, for $T<T c$, the system is in a ferromagnetic phase whereas, for $T>T c$ the system does not show any spontaneous magnetization, i.e., one has $M=0$ which means that the system behaves like a paramagnetic phase.

Interestingly, even in this case, near the critical point some thermodynamic quantities scale with one another as,

$$
C \sim|1-\tau|^{0}, \quad \chi_{T} \sim|1-\tau|^{-1}, \quad M \sim(H)^{3}, \quad M \sim \sqrt{|1-\tau|}
$$

Where, $X_{T}$ is the magnetic susceptibility. Quite remarkably, the critical exponents obtained for a magnetic system (using mean field theory) exactly coincide with those obtained for the van der Waals fluid with $\mathrm{H}$ taking the role of pressure and $\mathrm{M}$ taking the role of volume! Not only do the thermodynamic functions of two very different kind of systems scale in a similar fashion near their respective critical points, but the critical exponents are also the same. This prompted Landau to develop his famous phenomenological theory of phase transitions.

\section{Landau Theory}

Since the properties of a thermodynamic system behave in a rather universal manner near a critical point, Landau proposed a rather phenomenological approach towards dealing with systems near the critical point. As mentioned in the introduction, he defined a phase transition as a transformation of a system which brings about certain changes in the symmetry of the thermodynamic system. For instance, spins in a paramagnetic solid system are completely randomly oriented leading to a more symmetric but disordered phase with net zero spontaneous magnetization whereas those in a ferromagnetic system are rather more ordered leading to a rather less symmetric but an ordered phase with net non-zero spontaneous magnetization. The main ingredient in Landau's formulation is that of an order parameter which characterizes the difference between the ordered and disordered phases. Clearly, for the magnetic case, the magnetization $\mathrm{M}$ can be taken to be the order parameter which is zero in the disordered paramagnetic phase whereas it is non-zero in the ordered ferromagnetic phase. 
The central idea of this phenomenological approach is to assume that in the ordered phase where the order parameter is non-zero, the free energy is an analytic function of the order parameter such that it can be expanded as a power series,

$$
F=F_{0}(T)+a M+b M^{2}+c M^{3}+d M^{4}+\cdots
$$

Where, $F_{0}(T)$ is the free energy of the disordered phase and a,b,c,d,... are coefficients which are not all necessarily positive. Since the order parameter vanishes at the critical point, in its vicinity it is small and thus it suffices to retain only the first few terms in the expansion. Once this expansion is made near the critical point, one can consider minimizing the free energy to find equilibrium configuration of the system.

The authors will now apply it to the case of a magnetic phase transition discussed in the last section. To do so, let them note that the ferromagnetic phase is symmetric under the transformation $M \rightarrow-M$ which means that the system magnetized in the positive $z$-direction is as good as the system magnetized in the negative z-direction. This is most clearly seen from the fact that the magnetic system admits both $\pm M_{0}$ as its solutions below the critical point. Thus, the authors need not retain the odd power terms in the free energy expansion which means they can write the free energy in powers of the magnetization as,

$$
F=F_{0}(T)+\alpha M^{2}+\beta M^{4}
$$

for two coefficients $\alpha$ and $\beta$. Minimization of the free energy gives,

$$
\frac{\partial F}{\partial M}=0=\alpha M+2 \beta M^{3}
$$

This gives as solutions $M=0, \pm M_{0}$ were,

$$
M_{0}=\sqrt{-\frac{\alpha}{2 \beta}}
$$

Then, taking $\alpha<0$, one can obtain real values of $M_{0}$. Since, the magnetization is only non-zero in the ordered phase which occurs for $T<T_{c}$, it is convenient to define $\alpha=\left(T-T_{d}\right) \alpha^{\prime}$ for some positive constant $\alpha^{\prime}$. This means that for $T>T_{c}$ the only real point at which the free energy has an extremum is $M=0$. Thus, all the basic features of the ordered and disordered phases can be summarized from the free energy expansion,

$$
F=F_{0}(T)+\alpha^{\prime}\left(T-T_{c}\right) M^{2}+\beta M^{4}
$$

which has been plotted in figure 2 .

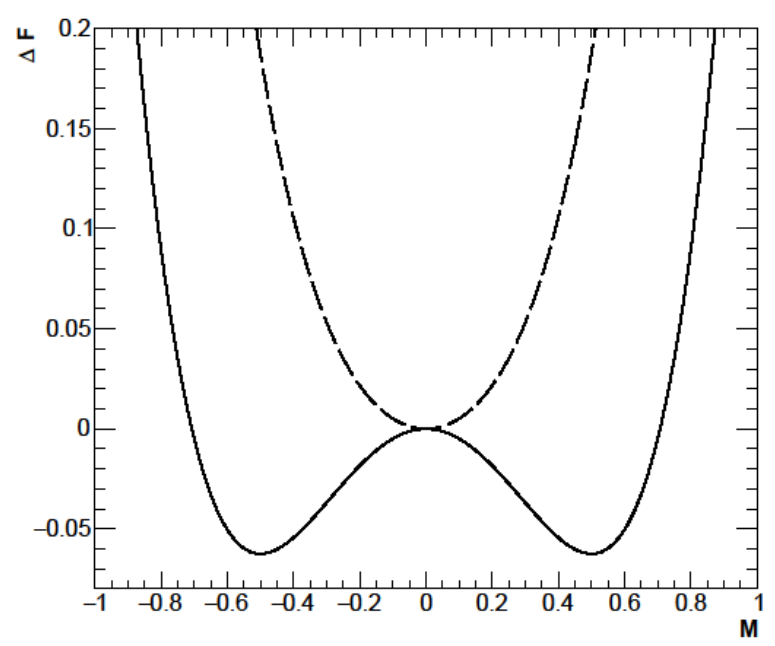

Figure 2: The free energy difference $\Delta F=F-F_{0}$ vs $M$ for $T>T_{c}$ (dotted line) and $T<T_{c}$ (solid line), $\alpha^{\prime}=1$ and $\beta=1$ in appropriate units. 
Next, they must check if the scaling of thermodynamic quantities near the critical point can be reproduced using Landau's theory. For example, in the ordered phase, they have (for $T<T_{\mathrm{c}}$ ),

$$
C(T)=-T \frac{\partial^{2} F}{\partial T^{2}}=C_{0}(T)+\frac{2 \alpha^{\prime 2} T}{\beta}
$$

where $C_{0}(T)$ is the specific heat of the disordered phase which means that there is a discontinuous jump of the specific heat at the phase transition by the amount $\Delta C=\frac{2 \alpha^{\prime 2} T}{\beta}$. This implies that it does not go to zero or infinity scaling with the dimensionless temperature $\tau$ thus giving . $C \sim|1-\tau|^{0}$. Furthermore, it can be shown that below the critical point,

$$
\chi_{T}=\frac{1}{4 \alpha^{\prime}\left(T-T_{c}\right)} \sim \frac{1}{|1-\tau|}
$$

once again exactly matching their expectations. Similarly, one can also derive the other scaling relations. Thus, several aspects of the paramagnetic-ferromagnetic phase transition can be exactly determined from the simplified phenomenological model of Landau where one simply needs to expand the free energy of the ordered phase in powers of the order parameter. Although this model is over simplified, it does provide one with a good description up to the first approximation of a phase transition.

\section{DISCUSSION}

The authors now point out to limitations of such a phenomenological approach. The most obvious limitation of such an approach was already encountered in one of the theories discussed above where mean field theory (equivalent to Landau theory) which works for any number of dimensions indicates towards the existence of a phase transition even in one dimension whereas the exact solution to the Ising model in one dimension tells them that there can be no such phase transition. This happened because mean field theory is only an approximate method and the approach of coarse graining the interactions give them the wrong answer in one dimension. Furthermore, in two and three dimensions, although mean field theory gives us qualitatively correct results, it produces the critical exponents different from that observed in the experiments. The physical explanation for this is that replacing the actual interactions between the degrees of freedom of a system with effective or coarse-grained interactions is not always a very good approximation. This leads to the breakdown of Landau's theory although it works very well in many other cases. In fact, for systems with number of dimensions more than four, Landau theory gives very good results. This dimension $d=4$ is known as the upper critical dimension and the fact that Landau theory works well for dimensions greater than four is the famous Ginzburg criterion for the validity of Landau's formalism. Nevertheless, for lower dimensions $d>1$, even if mean field theories predict the wrong critical exponents, it does provide a decent qualitative picture and a good first approximation to phase transitions. It is this reason why such phenomenological formulations are of interest to scientists even today.

An aspect which was ignored is related to the previously pointed limitation is that in Landau's original formulation, one does not consider the possibility of fluctuations of the order parameter. This is a serious drawback because close to the critical point, there might be strong fluctuations in the order parameter, and it cannot possibly be taken to be homogenous throughout the system as was considered in the last section. One way to explicitly incorporate fluctuations is to consider gradient terms in the free energy expansion and this strategy has been adopted in several cases. For example, if the system has one scalar order parameter, say $\eta$ (not necessarily magnetization) which is allowed to have spatial fluctuations in d-dimensions, the free energy is given by (up to some geometric factors), 


$$
F=\int d^{d} r\left[f_{0}(T)+\gamma(\nabla \eta)^{2}+\left(T-T_{c}\right) \alpha \eta^{2}+\beta \eta^{4}\right]
$$

Where, $f_{0}(T)$ is the free energy density of the disordered phase. Clearly, $\gamma=0$ means neglecting spatial fluctuations in $\eta$. Perhaps the most famous examples of formalisms incorporating fluctuations in the order parameter are the Landau-Ginzburg theory (Ginzburg, 2009) for superconductors and superfluids as well as the Landau-de Gennes theory (De Gennes \& Prost, 1993) for liquid crystals. Although these methods provide a better way to deal with phase transitions and critical phenomenon, they are still limited due to their phenomenological origin and a much more robust technique can only be understood from the point of view of re-normalization group theory (Goldenfeld, 2018).

\section{CONCLUSION}

In this short article, they have discussed some elementary aspects of the theory of phase transitions. Generic properties of a liquid-gas phase transition near the critical point bear a striking resemblance with that of a magnetic system near the critical point. This motivates the fact that perhaps many quantitative and qualitative aspects of phase transitions can be derived from a rather phenomenological set up, the simplest example of which was discussed above in one of the theories. This might be generalized to several directions in the study of many different types of phase transitions.

\section{ACKNOWLEDGEMENT}

The authors express their sincere thanks to the Department of Biotechnology, Government of India for funding from DBT-STAR College grant, under which this review was conducted. The authors are also grateful to the Principal and the DBT-STAR Coordinator, Surendranath College, for their support and encouragement in implementing this review at undergraduate level.

\section{REFERENCES}

De Gennes, P. G., \& Prost, J. (1993). The physics of liquid crystals (No. 83). Oxford university press.

Ginzburg, V. L. (2009). On superconductivity and superfluidity: a scientific autobiography (pp. 113-137). Berlin: Springer.

Goldenfeld, N. (2018). Lectures on phase transitions and the renormalization group. CRC Press.

Zemansky, M. W., \& Dittman, R. H. (1998). Heat and thermodynamics. 


\title{
Characteristics of the Detectors for Nuclear Radiation
}

\author{
Indranil Sadhya, Jamal Uddin, Mihir Kumar Das, Naved Akram, Nilay Sankar Chattopadhyay, \\ Prasanna Kumar Mondal* \\ Department of Physics, Surendranath College, Kolkata, India
}

*Corresponding Author's Email: prasanna.kumar.mondal@gmail.com

\begin{abstract}
The radiation characterization technique has undergone rapid transformation over the years. Earlier it was only possible to record the number of events in a certain detector, but now it is quite common to record the spectral data with digital computers. Now we can process information from the detector in a highly automated manner. A review of nuclear radiation detector with particular emphasis on description of the detector is given in this article. Here many types of detectors will be discussed in terms of its principle of operation and application. It is convenient to consider first the detector in which radiation interacts. The detectors use one of the two important processes by which the radiation transfers its energy to a material, i.e., excitation and ionization. One of the most useful detectors is scintillation counter which uses the light emitted when charged particles pass through certain materials. The basic process here is excitation. Other type of detectors makes use of ionization produced by charged particles passing through the material. This type of detector includes semiconductor detectors, Geiger-Muller counters, ionization chambers, and proportional counters. We know that if the incident radiation consists of charged particles (alpha particles, electrons, etc.) ionization is produced directly. However, the uncharged particles (gamma rays, neutrons, etc.) must first interact with the detector to produce charged particles. In this article an attempt has been made to cover most of the commonly used detector types. Also, the practical applications of the detectors are discussed.
\end{abstract}

\section{Keywords: Scintillation Counter; Semiconductor Detector; Geiger-Muller Counter; Ionization Chamber; Proportional Counter}

\section{INTRODUCTION}

It is well known that the ionizing radiation (such as cosmic rays, natural radioactive materials etc.) has always been present around us. However, the doors to this field of science were opened by Wilhelm Conrad Röntgen (Underwood, 1945) in 1895 and by Henri Becquerel (Myers, 1976) in 1896. In principle, the tools for the detection of ionizing radiation were existed before that! The thermoluminescence phenomenon (Daniels, Boyd \& Saunders, 1953) was described in $17^{\text {th }}$ century, the gold leaf electroscope was invented in 18th century and photography was developed during early 19 th century. Since the discovery of X-rays in 1895 the ionizing radiation has played an important role in different field of science.

Radiation is a form of energy, which can be entirely or partly deposited in a medium and thus produce an effect inside the medium. The detection and measurement of radiation (Knoll, 1979) depends on the detection and measurement of its effects in the medium. The most common types of ionizing radiation are alpha particle, beta particle, gamma-ray, X-ray, and neutron. The ionizing radiation can cause neutral atom or molecule to acquire electrical charge. Charged particle radiation (for example alpha or beta particles) has direct ionizing effect, whereas neutral radiation (for example gamma-ray, X-ray, or neutron) has an indirect ionizing effect. These radiations first produce charged particles which then have the ionizing effect.

Radiation detectors use a wide range of different physical and chemical interactions such as electronic charge collected from ionization produced in air, color change due to changes in atomic electronic states etc. (Flakus, 1982). The complexities of the different physical and chemical effects that are commonly used in radiation detectors make this subject very challenging. In many applications the object is to 
obtain the energy distribution of the radiation. The field of nuclear radiation detector is enormous, and a thorough systematic review is impractical and beyond current space limitations. In this present review we concentrate on the physical principles of operation of the major types of radiation detector such as the scintillation counter (Pringle, 1950), semiconductor detector (Sellin, 2003), Geiger-Muller counter (Trenn, 1986), ionization chamber (DeWerd \& Smith, 2021) and proportional counter (Curran, 1958). Although it is not necessary to be familiar with details of electronic circuits used in the detectors, it is essential that we must understand the functions and limitations of this system.

\section{LITERATURE REVIEW}

\section{Detection and Measurement of lonizing Radiation}

\section{Scintillation Counter}

The scintillation counter or scintillation detector is a device that is used for the detection of nuclear radiation by means of the scintillation effect (Pringle, 1950). The flash of light which is produced in a material due to the passage of a radiation (such as electron, alpha particle, other ion, or photon) is called scintillation. This was one of the earliest methods used for detection of charged particles where the scintillations produced in a phosphor screen were counted. Rutherford employed this device in his famous alpha-particle scattering experiment. The modern scintillation counters are more reliable electrical counters, which use high-gain photomultiplier tube (PMT) (Lubsandorzhiev, 2006) in combination with various scintillating material.

The schematic diagram of a scintillation counter is shown in figure 1. Here the energy of the incident radiation is converted to photons inside the scintillator. The reflector and optical coupler ensure that the photons are efficiently directed to the photocathode. The photocathode converts the light energy to a burst of photoelectrons. This follows an electrostatic focusing electrode that collects the electrons and then focuses them on the first dynode of the photomultiplier tube. The cascade of dynodes acts as electron multipliers which increase the number of electrons by production of secondary electrons (Knoll, 1979). The current gain for a standard 10 -stage multiplier is about $10^{6}$. The secondary electrons finally arrive at the anode and here a negative output pulse of the few millivolts' amplitude is usually generated. The rise time of such pulse depends on the lifetime of the excited state of the scintillator and on the time spread introduced in the PMT.

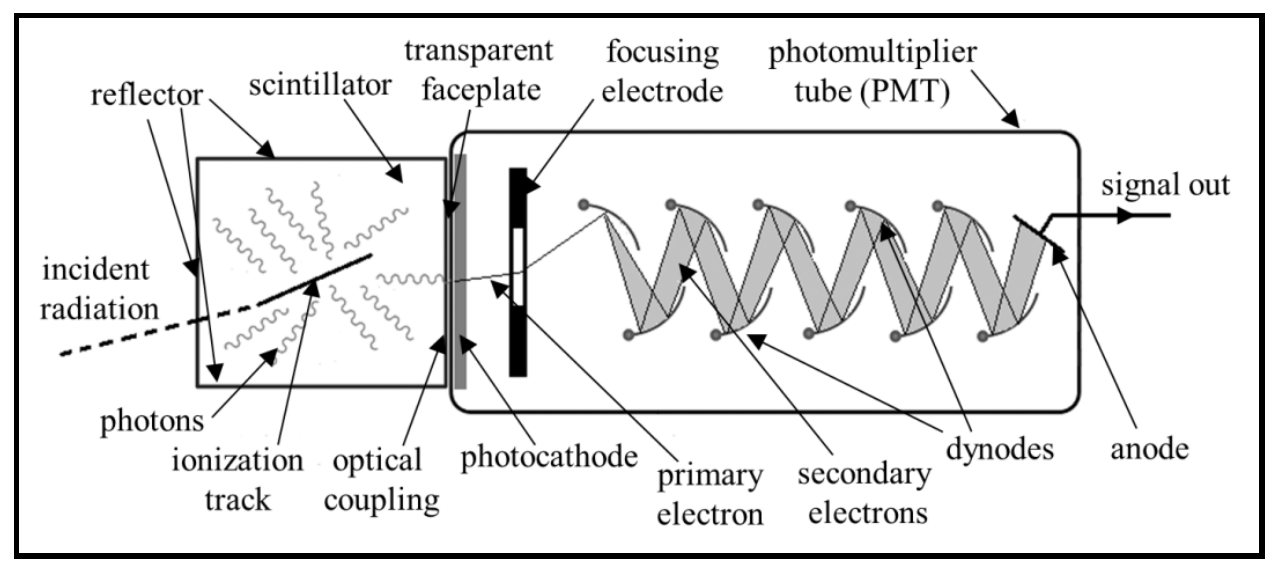

Figure1: Schematic Diagram of the Scintillation Counter

The scintillation counters are used for the detection of beta-ray, beta spectrometry (Salonen, 2006), detection of charged particles (Sherr, 1947), detection of gamma-ray, gamma spectrometry (Moszyński, 2003) etc.

Organic scintillators (Horrocks, 1971) are widely used for the detection of beta-rays because of their low effective atomic number, which effectively decreases the probability of backscattering. Due to the backscattering a beta particle may scatter out depositing only a small fraction of its energy in the scintillator. This effect is large at low beta energy and for high atomic number scintillator. Another 
advantage of organic scintillator is that due to their low gamma-ray sensitivity, it can efficiently detect beta particles in presence of gamma-rays. The organic scintillators may be divided into two classes, single crystals, and solutions (it also includes solid solutions, i.e., plastic materials).

Anthracene, P-terphenyl, P-quaterphenyl are common example of single crystal scintillator (Zaitseva et al. 2011). The liquid and plastic scintillators (Bertrand, Hamel \& Sguerra, 2014) have an advantage that they can be easily fabricated in large volumes. They may be prepared from two or three components. The bulk material is the solvent in which the scintillating substance (primary solute) is dissolved and usually another scintillator (secondary solute, called the wavelength shifter) is also included. An example of solution scintillators is p-terphenyl (primary solute) in toluene solvent along with 1,4-Bis(5phenyl-2-oxazolyl) benzene as the secondary solute. The concentration of solute in solution scintillators is usually kept low and the choice of solvent depends on the application. Usually, xylene is preferred because for a given solute it yields the largest pulse height. On the other hand, toluene exhibits a much smaller absorption of fluorescent light and commonly used when large volume is needed.

The organic scintillators are also useful for the measurement energies of beta-ray. They typically give a linear pulse height-energy curve down to about $100 \mathrm{keV}$; and the response below this is also nearly linear but with a different slope. For mono-energetic electrons the organic scintillators give Gaussian shaped pulse whose width inversely varies with the square root of the energy.

The modern scintillation counters have also made important contribution in the field of detection of gamma-radiations (Moszyński, 2003). The high-density solid gamma-ray scintillators give a far greater stopping power for photons than gas-filled counters. Advanced scintillation counters can also measure the gamma-ray energy spectra. The high density and high atomic number inorganic scintillators are very effective for gamma-ray detection. Although there are many inorganic scintillators, only the activated alkali halides can be grown in large single crystals, and they are also transparent in nature. Sodium iodide activated with TII is a widely used alkali halide scintillator. For the thallium doped sodium iodide $\mathrm{Nal}(\mathrm{TI})$ the light output per MeV is the largest compared to any other known scintillator (Kim et al. 2000). The relatively low-cost single crystal $\mathrm{Nal}(\mathrm{TI})$ with high luminescence efficiency can be obtained in wide variety of sizes and geometries which make it the most widely used scintillator.

The modern scintillation counter has also found its place in detection and spectrometry of charged particles. For the detection of charged particles ZnS activated by Ag can be used (Bodewits et al. 2016). $\mathrm{ZnS}(\mathrm{Ag})$ is available in the form of multi-crystalline powder, and thus although its light output is high its light transmission is poor. It is not suitable for measuring energy spectra of charged particles. To find the energies of charged particle we need to use some other inorganic scintillator, like the $\mathrm{Nal}(\mathrm{TI})$ or CsI(TI) which may be prepared in the form of thin clear disks.

The scintillation counters have many advantages over gas counters. They not only detect a particle but may also be used to find the rate of energy loss if the scintillator is thick enough (Paul, 1971). With the suitable combination of scintillator and photomultiplier tube (PMT) the scintillation counter is most suited in applications where high counting rate is required.

\section{Ionization Chamber}

The ionization chamber is also a very important class of detector that makes use of the ionization produced in a gas. It collects either the electron produced in the gas filled detector, or collects the ion pair i.e., the electron and positive ion both. As charged particle transverses through a material, it slows down due to loss of kinetic energy either by collisional or radiative energy loss. The total stopping power $(\mathrm{dE} / \mathrm{dx})$ has two components: the electronic energy loss due to Coulomb interactions and the nuclear energy loss due to nuclear interactions and emission of Cerenkov or Bremsstrahlung radiation (Knoll, 1979). The ionization completely removes the electron from the atom resulting production of an ion pair, i.e., the free electron and the positively charged atom from which the electron was removed. On the other hand, excitation raises an electron to a shell of higher energy only. The term $(d E / d x)_{\mathrm{c}}$, for only Coulomb interactions is known as the linear energy transfer (LET), i.e., the rate of energy loss of a charged particle due to ionization and excitation. Another quantity used for describing the energy loss is 
specific ionization (Brode, 1939), defined as the number of ion pairs formed per unit distance traveled by the charged particle. Specific ionization can be expressed as $(\mathrm{dE} / \mathrm{dx}) / \mathrm{w}$, where $w$ is the average energy required to produce one ion pair. The value of $w$ is usually constant for gases.

The behavior of the free electrons and positive ions depends on the nature of the gas and the electric field present in the detector. The drift velocity of electron depends on the type of gas used, gas pressure, and electric field strength. The positive ions move much more slowly through the gases compared to the electrons. We know that the free electrons may form negative ions by attaching themselves to neutral atoms or molecules. It is very harmful in ionization chambers using fast electron collection. In ionization chambers usually some rare gases, carbon dioxide, nitrogen, hydrogen, methane etc. are used.

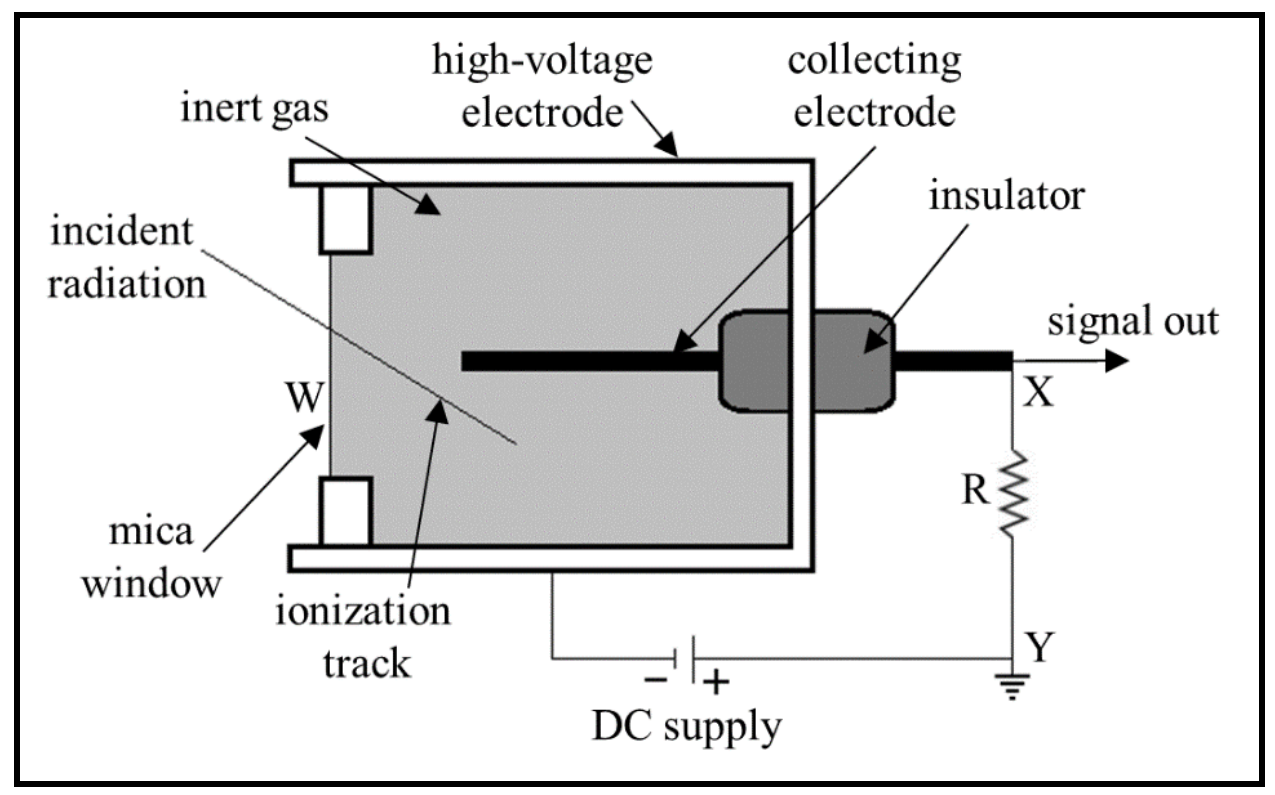

Figure 2: Schematic Diagram of the Ionization Chamber

There may be pulse-type chambers and current chambers. The main components of a gas filled ionization chamber are two insulated electrodes kept inside a gas filled vessel. The electrodes are maintained at few hundred to few thousand volts inside a suitable gas in which ions have rather long lifetime. Usually, the chambers possess parallel plate geometry or cylindrical geometry. In parallel plate chambers two parallel plates are separated by a distance. In cylindrical chambers a conducting cylindrical shell with a coaxial insulated metallic wire acts as electrodes. The construction of a typical ionization chamber is shown schematically in figure 2 . Here one electrode is the outer metallic cylinder that is connected to the negative terminal of the DC power supply and the second electrode is the coaxial central wire connected to the positive terminal of the DC supply through a resistor $\mathrm{R}$. To increase the detector sensitivity the gas is maintained under some pressure; it ensures more targets for the incoming radiation. The thin mica window (W) enables the incoming charged particles to enter the chamber easily. The ion pairs produced inside the chamber are attracted towards different electrodes due to the presence of the electric field between them. As a result of this, voltage pulse is developed between points $X$ and $Y$, which is then amplified and finally recorded for further analysis. The ionization chamber detects the bursts of particles and can discriminate pulses developed due to different radiations (for example alpha and beta particles).

The ionization chambers can be used in current or pulse mode. In contrast the Geiger-Muller counters or proportional counters are usually operated only in pulse mode. The ionization chambers can be used for the detection of alpha and beta particles, charged particles (Göpfert, Hambsch \& Bax, 2000), gamma rays (Fares et al. 2020) (survey meters and for environmental monitoring), neutrons (Salvat et al. 2012) etc. They are used for measuring the strength of sources of heavy charged particles. A simple ionization chamber can be employed for normal alpha counting operation. The parallel-plate type pulse chambers are commonly used for measuring the fission cross-section and for comparison of fissile sources. The 
current type chambers are particularly suitable to the assay of gamma-ray sources. The ionization chambers can also be used as simple handheld survey instruments (Sankaran \& Pillai, 1981) to measure low or high exposure rates, and often used in dosimetry applications. The gridded ionization chambers are commonly used for analyzing charged particle energy spectra (especially alpha particles). Very good energy resolution can be obtained with large-area sources.

The working of an ionization chamber depends on the amount of charge collected when a radiation passes through it. No internal amplification happens within the ionization chamber, and one need to use external amplifiers for enhancing the signal. Whereas the proportional counter and Geiger-Muller counter make use of gas multiplication (Sikkema, 1970) as a result they can produce output signals of amplitude much higher than that of ionization chamber.

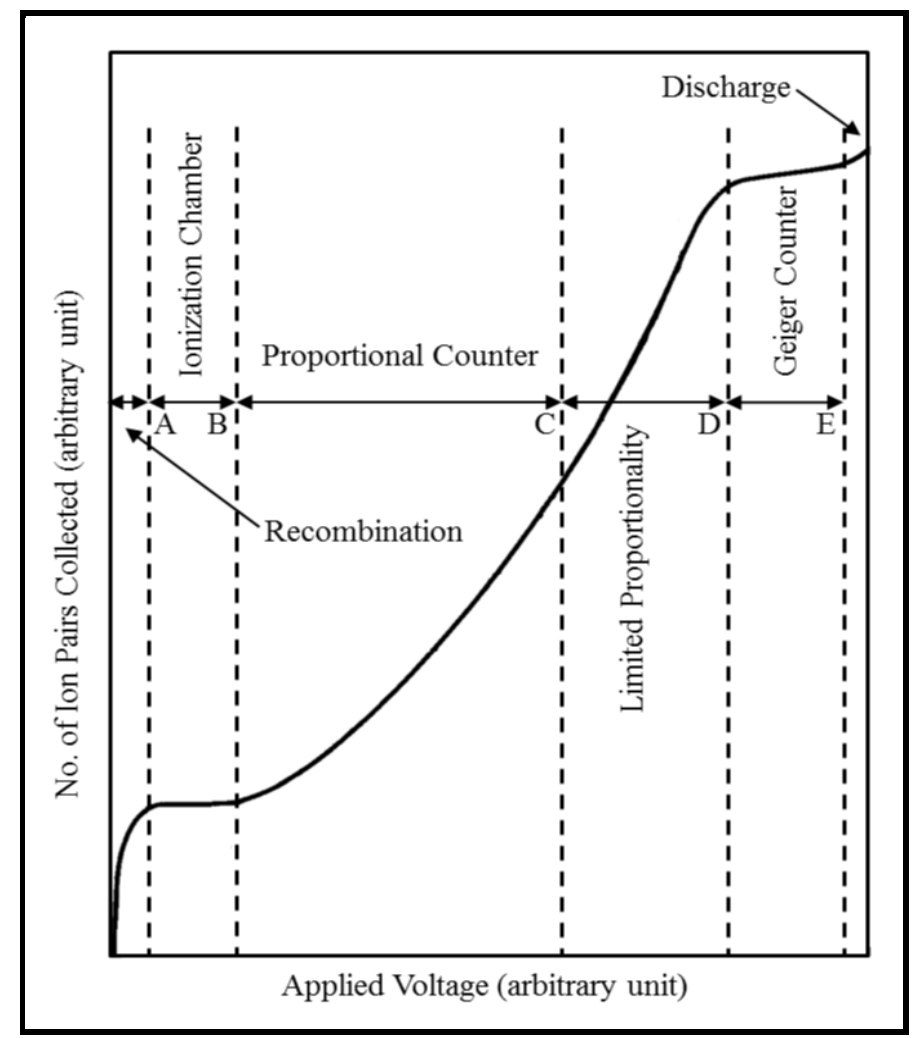

Figure 3: Number of Ion Pairs Collected in a Counter as a Function of the Applied Voltage

The difference between simple ionization chamber and gas multiplication counter may be understood from the variation of number of ion pair (or the pulse height) collected as a function of the applied voltage plot (figure 3). As evident from the figure, the number of ion pairs collected increases with applied voltage, until a saturation region $(A B)$ is reached. After that the electrons (i.e., primary electrons) acquire enough energy near the anode to produce additional ionization. Throughout the region $\mathrm{BC}$ each primary electron produces an avalanche of $\mathrm{M}$ number of secondary electrons ( $\mathrm{M}$ is the gas multiplication). In region $B C$ the pulse height is proportional to the initial ionization and the proportional counter operates in this region. A further increase in applied voltage creates more and more secondary ion-pairs near the anode such that the positive space charge starts to interfere with the formation of avalanche by neighboring electrons. As a result, different amount of primary ionization produces pulse of slightly different amplitude, but the strict proportionality is lost (region CD). Region DE is the Geiger-Muller region where the detector again produces pulse of almost constant amplitude. In this region a single electron initiates an avalanche which propagates throughout entire length of the anode.

\section{Proportional Counter}

The proportional counter consists of a gas filled chamber having a thin coaxial wire that acts as anode and the metal outer vessel acts as the cathode. A sufficiently high voltage is applied between the electrodes such that it does not produce any discharge. The device has a thin mica window through 
which the incident radiation enters the chamber and produces electron-positive ion pair. The electrons are attracted towards the anode, near which the electric field is very high compared to the other region. Near the anode the electrons (primary) gain sufficient kinetic energy and collide with other gas molecules and produce more ion pairs ( $10^{4}$ to $10^{6}$ times more secondary ion pairs can be produced). This mechanism of producing more ion pairs is known as the gas multiplication. In figure 3 , the region $\mathrm{BC}$ represents the proportional counter region. Here the output signal is proportional to the number of ion pairs produced and thus the detector is called proportional counter.

To achieve the gas multiplication, one need to provide an electric field suitable for accelerating the primary electrons to an energy that can produce additional ionization. If $a$ and $b$ are the radii of the anode wire and the cylindrical cathode respectively, then the electric field $(E)$ at a radial distance $r$ is

$$
E=\frac{V}{r \ln (b / a)}=\frac{\mathrm{C}}{r}
$$

where $V$ is the potential difference between the electrodes and $C$ is a constant (Knoll, 1979). It is evident from Eq. (1) that the high field is confined to the region near the anode wire. The time required to collect all the ions usually depends on the applied voltage, geometry of the detector, nature of the gas, and internal pressure. In a typical proportional counter, the collection time is generally a few hundred microseconds. The gas multiplication factor of the detector could be as high as about $10^{6}$ for low primary ionizations.

The proportional counter can be used for the detection of various types of ionizing radiation, for example detection of alpha-particles, beta-particles, gamma-rays etc. The efficiency of the detector is high for beta, but lower for alpha particles. They can be used for discrimination between the alpha and beta radiations. Due to their shorter resolving time, the proportional counters can be used at much higher counting rates than the Geiger-Muller counter. For the detection of alpha and beta particles the detector must be provided with a thin window. The gamma-rays and X-rays can easily penetrate the metal walls of the chamber and thus windowless proportional counters may be used to detect gamma-rays and Xrays (Agrawal et al. 2017). Proportional counters are also used for alpha and beta spectroscopy. For the low energy beta-ray and X-ray spectrometry the proportional counter is capable of much better resolution than scintillation counter. Large volume spherical shaped proportional counter with nitrogen gas is used for the detection of neutrons (Bougamont et al., 2017) via the reactions ${ }^{14} \mathrm{~N}(n, p){ }^{14} \mathrm{C}$ and ${ }^{14} \mathrm{~N}(n, \alpha) 11 \mathrm{~B}$.

\section{Geiger-Muller Counter}

The Geiger-Muller counter or Geiger counter is the most popular type of radiation detector among the gas-filled detectors. The Geiger counter can detect almost all ionizing radiation with sufficient sensitivity. Also due to its large pulse amplitude it can be used without a high-gain amplifier. The Geiger-Muller counter cannot be used in spectrometry, but due to other advantages it is very useful for other applications where simple counting is required such as for survey devices and other field applications. The Geiger-Muller counters (Knoll, 1979) are like the proportional counters, but they differ in nature of the filling gas and the gas pressure. In almost all Geiger-Muller counters a cylindrical geometry is used because here the necessary parameters can be controlled easily. This detector is very easy to construct and is extremely sensitive to charged particles.

When the voltage applied to a proportional counter is increased, at some point the pulses become of same amplitude (figure 3). This region of operation where the pulses do not depend on the initial ionization is called the Geiger region. A simple Geiger counter consists of a cylindrical metallic tube (cathode), inside which a fine tungsten wire (anode) is kept along the axis of the tube and this system is mounted inside a glass tube. The dimension of the tube varies depending on the application. The detector is filled with some inert gas together with a quenching agent that quenches the initial discharge. When a positively charged gas ion strikes the cathode it may produce secondary electron which result in further discharge unless these multiple discharges are not controlled by some quenching agent.

In Geiger-Muller counter the electrodes are so shaped that even with a moderate applied voltage a high 
electric field is produced near one of them. The central electrode is so thin that a high electric field is produced around it. Like the proportional counter the electrons drift towards the anode and accelerated in the electric field resulting release of more electrons by ionization and an avalanche occurs. Here each secondary electron produces more ionization, and some excited atoms also produce photon and sometime photoelectron. With the increase of electric field, the number of photons increases until a photoelectron is produced in each avalanche. Here the avalanche region spreads until it engulfs the entire central wire and an amplification as high as $10^{8}$ can be achieved. The initial ionization thus produces a very high amplitude pulse.

Almost all Geiger-Muller counters are self-quenching (Carver \& White, 1949) in nature. Argon filled counters can have alcohol as the quenching gas. Here argon ion collides with argon atoms and alcohol molecules. The probability that an argon ion will be neutralized in an encounter with an alcohol molecule is very high compared to the opposite transfer of charge. Thus, the positive ions which reach the cathode are composed only of alcohol ions. These ions cannot produce secondary electrons and are neutralized at the cathode.

Unlike the proportional counter the Geiger tube does not immediately recover from the discharge (Knoll, 1979). In Geiger tube the positive ions form a cylindrical capsule around anode wire that disrupts the electric field. As a result of this the starting of new avalanche near the anode becomes difficult. This continues until the ions are migrated out of the high electric field region. The time interval during which the tube remains insensitive to additional ionization is called the dead time. The dead times for Geiger counters are usually in the range 100 to $300 \mu \mathrm{s}$. After that once the detector starts to count again it requires some more time before the pulse size returns to the original amplitude. This time interval is known as the recovery time. In a practical counter it is required to know the resolving time $(\tau)$, which is the average time interval for which the system is insensitive. To obtain the true counting rate of a Geiger counter the resolving time should be known. If $\mathrm{n}$ is the measured counting rate of the detector, then the corrected counting rate $\mathrm{N}$ can be determined from the following equation, $N=n /(1-n T)$.

The Geiger-Muller counters are not as stable as the proportional counters, they also possess a long dead time, and they produce pulses of constant amplitude, which is independent of the initial ionization, and therefore cannot distinguish between different radiations. It is usually designed for the detection of alpha particles, beta particles, gamma rays (Ivasheva, Borisov \& Semenikhin, 2018) and X-rays (Coyle, Hale \& Wainwright,1953). The Geiger-Muller counters are widely used in cosmic ray detection (Wibig et al. 2006), where multiple detectors are operated in coincidence or anti-coincidence arrangement (Altameemi, Hamid \& Kamil, 2021). They also have extensive applications in radiation dosimetry, radiological protection, and nuclear industry.

\section{Semiconductor Radiation Detector}

The semiconductor radiation detector or the solid-state detector is detector in which a semiconductor material such as silicon or germanium crystal used as the active medium (McKenzie,1979; Sellin, 2003). The use of $p-n$ junction is the most important innovation in radiation detectors since the development of the scintillation counter. The semiconductor detector is quite like the gas ionization chamber, except that here the charge is carried by electron-hole pair instead of electron-positive ion pair. For this reason, these detectors are also called solid-state ionization chamber. In semiconductor detector the sensitive layer can be made very thin with a high stopping power. It is also intrinsically highspeed device due to the high mobility of the charge carriers in the electric field, coupled with the small distance between the electrodes. The energy required to produce one electron-hole pair is very low $(\sim 3.5 \mathrm{eV}$ for $\mathrm{Si})$ for this detector, which makes it better than the gas filled counter.

To collect all the produced charges the parallel-plate ionization chamber with small electrode spacing requires a uniform and high electric field. For this the resistivity of the material need to be very high. This makes large power dissipation and a random fluctuation in the current which is usually large in comparison to the actual signal produced due to the collection of electron-hole pairs at the electrodes. Therefore, we need to find some other way of producing a high electric field inside without using material 
of very high resistivity and without applying high voltages. One possible way is the use of reversed biased, p-n junction (Knoll, 1979).

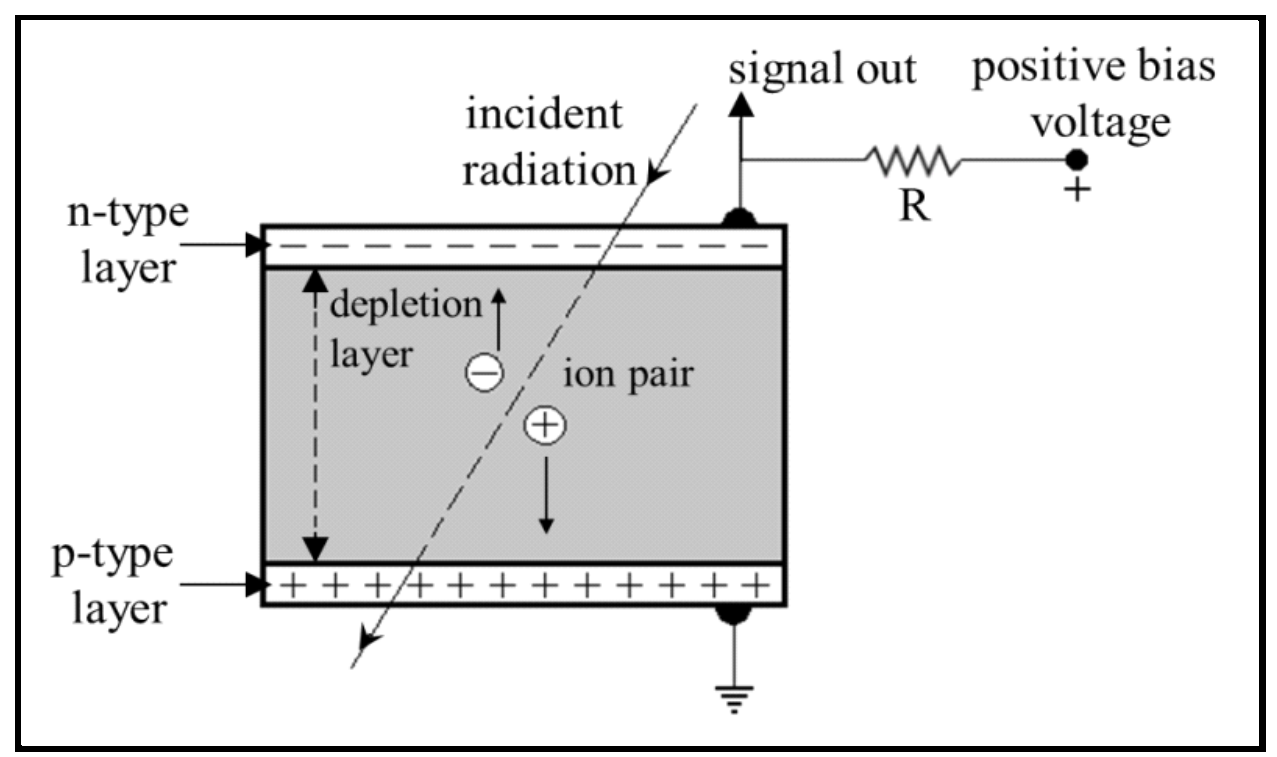

Figure 4: Schematic Diagram of The Semiconductor Detector

The schematic diagram of a typical semiconductor detector is shown in figure 4 . If a voltage is applied by connecting the negative terminal to the $\mathrm{p}$-type region and the positive terminal to the $n$-type region, the $p-n$ junction is said to be reverse biased. The barrier height increases with the increase in the reverse bias voltage. If the applied voltage and the resistivity of the material are known, the barrier height may be determined. So, we can estimate the required depth of the region to be certain that the incident particles will be stopped within the sensitive part of the detector. In semiconductor detector the electrons and holes move away from the junction region and form a depletion layer with no charge carriers there. As the resistivity of the depletion layer is high a large potential difference can be maintained across it, and it works like a solid ionization chamber. The thickness of the depletion layer ranges from few hundred micrometer to few millimeters.

The charged particles while passes through the depletion region produce electron-hole pairs. These electrons move to the conduction band and can move freely through the semiconductor crystal. On the other hand, the holes remaining in the valence band can also move freely. As a result of these a current pulse is generated, the amplitude of which is proportional to the number of electron-hole pairs produced. So, the output signal measured across the resistance $R$ depends on the energy deposited by the incoming radiation. Most of the modern semiconductor detectors are of surface barrier type. One way of making such a detector is by diffusing an n-type impurity to a depth of about $1000 \mu \mathrm{m}$ from the surface of the semiconductor having a small concentration of a p-type impurity. As a result, the p-n junction is established just below the surface of the active material. The semiconductor detectors are operated at very low temperature (liquid nitrogen is usually used for this purpose) to reduce the electrical noise. A very thin layer of good electrical conducting material (such as gold) is applied on the surface of the detector to ensure a good electrical contact and at the same time not much affecting the entry of the incoming radiation inside the active region of the detector.

The output signal produced in the semiconductor detector is about 10 times the amplitude of the pulse obtained from the ionization chamber for the passage of same radiation. Therefore, in this type of detector, the statistical fluctuation is much lower compared to that in ionization chamber. This makes the semiconductor counter very sensitive, and it can give an extremely high counting rate (about 105 particle/sec). Among all types of radiation detectors, the semiconductor detectors have the best energy resolution and due to the very narrow depletion layer the rise time of output pulse is also very small (about $10 \mathrm{~ns}$ ).

The semiconductor detectors are very useful for the detection of heavy ionizing particles, for example 
the alpha-particles, protons, heavy ions, fission fragments etc. (Britt \& Wegner, 1963; Granja et al. 2018). But they are not very suitable for detecting gamma-rays since their specific ionisation is very small. The width of the depletion layer is usually not sufficient to stop the gamma-rays. For the detection of gamma-rays lithium drifting technique is used (one example is the lithium drifted germanium detector, GeLi) (Robinson et al. 1965).

The size of the semiconductor detectors can be made very small and can be used for studying the nuclear reactions. They can also discriminate the charged particles in a heavy gamma-rays or neutrons background. For particles with ranges smaller than the thickness of depletion layer, the detector can also act as a spectrometer (Ruddy, Seidel \& Sellin, 2009). In such application the pulse height is proportional to the kinetic energy of the incoming radiation.

\section{DISCUSSION}

The radiation detectors make use of different physical and chemical interactions such as electronic charge collected, color change etc. The involvement of the different physical and chemical effects makes this subject very exciting. The field of nuclear radiation detector is enormous, and a detail systematic review is difficult and beyond the current space limitations. Apart from the detectors described here, there are also other types of radiation detectors, such as the spark chamber (Rodphai, Suwonjandee \& Asavapibhop, 2018), cloud chamber (Alexandrov et al. 2017), bubble chamber (Behnke et al. 2013), superheated drop detector (Apfel R.E. 1979), Cherenkov counter, photographic detector etc. (Knoll 1979). They also have many applications but to keep this article short we did not discuss them here.

\section{CONCLUSION}

This review is focused on describing different technologies used for detecting the nuclear radiation. Here we have briefly explained the general characteristics and properties that are used to describe the performance and behavior of few common radiation detectors. We have covered scintillation counter, semiconductor detector, Geiger-Muller counter, ionization chamber, and proportional counter. These are the most used detectors, which are developed depending on the specific needs. The technological advancement in recent years has improved the radiation detectors. It is probably safe to say that the development of nuclear radiation detectors will continue and will find new applications.

\section{ACKNOWLEDGEMENT}

The authors express their sincere thanks to Department of Biotechnology, Government of India for the funding from DBT-STAR College grant, under which this review project was conducted. We are also grateful to the Principal and the DBT-STAR Coordinator, Surendranath College, for their support and encouragement in implementing this review project at undergraduate level.

\section{REFERENCES}

Agrawal, P. C., Yadav, J. S., Antia, H. M., Dedhia, D., Shah, P., Chauhan, J. V., ... \& Subbarao, K. (2017). Large area X-ray proportional counter (LAXPC) instrument on AstroSat and some preliminary results from its performance in the orbit. Journal of Astrophysics and Astronomy, 38(2), 1-13.

Alexandrov, A., De Lellis, G., Di Crescenzo, A., Lauria, A., Montesi, M. C., Pastore, A., ... \& Tioukov, V. (2017). Measurements of $12 \mathrm{C}$ ions beam fragmentation at large angle with an Emulsion Cloud Chamber. Journal of Instrumentation, 12(08), P08013.

Altameemi, R. N., Hamid, N. S. A., \& Kamil, W. M. A. W. M. (2021). Examination the cosmic ray muon attenuation by heavy metal alloys. In Journal of Physics: Conference Series (Vol. 1768, No. 1, p. 012006). IOP Publishing.

Apfel, R. E. (1979). The superheated drop detector. Nuclear Instruments and Methods, 162(1-3), 603608.

Behnke, E., Benjamin, T., Brice, S. J., Broemmelsiek, D., Collar, J. I., Cooper, P. S., ... \& COUPP 
Collaboration. (2013). Direct measurement of the bubble-nucleation energy threshold in a CF 3 I bubble chamber. Physical Review D, 88(2), 021101.

Bertrand, G. H., Hamel, M., \& Sguerra, F. (2014). Current status on plastic scintillators modifications. Chemistry-A European Journal, 20(48), 15660-15685.

Bodewits, E., Cester, D., Lunardon, M., Moretto, S., Schotanus, P., Stevanato, L., \& Viesti, G. (2016). Characterization of a large area $\mathrm{ZnS}(\mathrm{Ag})$ detector for gross alpha and beta activity measurements in tap water plants. IEEE Transactions on Nuclear Science, 63(3), 1565-1569.

Bougamont, E., Dastgheibi, A., Derre, J., Galan, J., Gerbier, G., Giomataris, I., ... \& Tsiledakis, G. (2017). Neutron spectroscopy with the Spherical Proportional Counter based on nitrogen gas. Nuclear Instruments and Methods in Physics Research Section A: Accelerators, Spectrometers, Detectors and Associated Equipment, 847, 10-14.

Britt, H. C., \& Wegner, H. E. (1963). Response of semiconductor detectors to fission fragments. Review of Scientific Instruments, 34(3), 274-277.

Brode, R. B. (1939). The specific ionization of high-speed particles. Reviews of Modern Physics, 11(3-4), 222.

Carver, J. H., \& White, G. K. (1949). Methylene Bromide as a Quenching Agent in Geiger-Müller Counters. Nature, 163(4144), 526-527.

Coyle, R. A., Hale, K. F., \& Wainwright, C. (1953). The design and operation of a precision Geiger-Müller counter X-ray diffraction spectrometer. Journal of Scientific Instruments, 30(5), 151.

Curran, S. C. (1958). The proportional counter as detector and spectrometer. In Nuclear Instrumentation II/Instrumentelle Hilfsmittel der Kernphysik II (pp. 174-221). Springer, Berlin, Heidelberg.

Daniels, F., Boyd, C. A., \& Saunders, D. F. (1953). Thermoluminescence as a research tool. Science, 117(3040), 343-349.

DeWerd, L. A., \& Smith, B. R. (2021). Ionization chamber instrumentation. In Radiation Therapy Dosimetry: A Practical Handbook (pp. 19-30). CRC Press.

Fares, M., Messai, A., Begaa, S., Messaoudi, M., Negara, K., \& Debili, M. Y. (2020). Design and study of the characteristics of a versatile ionization chamber for gamma-ray dosimetry. Journal of Radioanalytical and Nuclear Chemistry, 326(2), 1405-1411.

Flakus, F. N. (1982). Detecting and Measuring lonizing Radiation- A Short History. IAEA Bulletin, 23(4), 31-36.

Göpfert, A., Hambsch, F. J., \& Bax, H. (2000). A twin ionization chamber setup as detector for light charged particles with energies around $1 \mathrm{MeV}$ applied to the $10 \mathrm{~B}(\mathrm{n}, \mathrm{\alpha}) 7 \mathrm{Li}$ reaction. Nuclear Instruments and Methods in Physics Research Section A: Accelerators, Spectrometers, Detectors and Associated Equipment, 441(3), 438-451.

Granja, C., Jakubek, J., Polansky, S., Zach, V., Krist, P., Chvatil, D., ... \& Martisikova, M. (2018). Resolving power of pixel detector Timepix for wide-range electron, proton and ion detection. Nuclear Instruments and Methods in Physics Research Section A: Accelerators, Spectrometers, Detectors and Associated Equipment, 908, 60-71.

Horrocks, D. (1971). Organic Scintillators and Liquid Scintillation Counting. $1^{\text {st }}$ Edition. Academic Press. United States.

Ivasheva, A. Y., Borisov, V. S., \& Semenikhin, P. V. (2018, December). Adjusting of the detection efficiency of Geiger-Muller counters in the energy range of gamma radiation from 50 to $3000 \mathrm{keV}$. In Journal of Physics: Conference Series(Vol. 1135, No. 1, p. 012042). IOP Publishing.

Kim, J. H., Choi, Y., Joo, K. S., Sihn, B. S., Chong, J. W., Kim, S. E., ... \& Kim, B. T. (2000). Development of a miniature scintillation camera using an $\mathrm{Nal}$ (TI) scintillator and PSPMT for scintimammography. Physics in Medicine \& Biology, 45(11), 3481. 
Knoll, G. F. (1979). Radiation Detection and Measurement, Wiley \& Sons. New York.

Lubsandorzhiev, B. K. (2006). On the history of photomultiplier tube invention. Nuclear Instruments and Methods in Physics Research Section A: Accelerators, Spectrometers, Detectors and Associated Equipment, 567(1), 236-238.

McKenzie, J. M. (1979). Development of the semiconductor radiation detector. Nuclear Instruments and Methods, 162(1-3), 49-73.

Moszyński, M. (2003). Inorganic scintillation detectors in y-ray spectrometry. Nuclear Instruments and Methods in Physics Research Section A: Accelerators, Spectrometers, Detectors and Associated Equipment, 505(1-2), 101-110.

Myers, W. G. (1976). Becquerel's Discovery of Radioactivity in 1896. Journal of Nuclear Medicine, 17(7), 579-582.

Paul, J. M. (1971). The density effect and rate of energy loss in common plastic scintillators. Nuclear Instruments and Methods, 96(1), 51-59.

Pringle, R. W. (1950). The scintillation counter. Nature, 166(4209), 11-14.

Robinson, R. L., Stelson, P. H., McGowan, F. K., Ford Jr, J. L. C., \& Milner, W. T. (1965). Gamma-ray energies determined with a lithium-drifted germanium detector. Nuclear Physics, 74(2), 281-288.

Rodphai, N., Suwonjandee, N., \& Asavapibhop, B. (2018, December). Cosmic-Ray Muons Detection by a Spark Chamber. In Journal of Physics: Conference Series (Vol. 1144, No. 1, p. 012050). IOP Publishing.

Ruddy, F. H., Seidel, J. G., \& Sellin, P. (2009, October). High-resolution alpha spectrometry with a thinwindow silicon carbide semiconductor detector. In 2009 IEEE Nuclear Science Symposium Conference Record (NSS/MIC) (pp. 2201-2206). IEEE.

Salonen, L. (2006). Alpha/beta liquid scintillation spectrometry in surveying Finnish groundwater samples. Radiochemistry, 48(6), 606-612.

Salvat, D. J., Morris, C. L., Wang, Z., Adamek, E. R., Bacon, J., Hickerson, K. P., ... \& Young, A. R. (2012). A boron-coated ionization chamber for ultra-cold neutron detection. Nuclear Instruments and Methods in Physics Research Section A: Accelerators, Spectrometers, Detectors and Associated Equipment, 691, 109-112.

Sankaran, A., \& Pillai, K. M. (1981). A Multiplication lonization Chamber Survey Meter for Low Level X and Gamma Ray Monitoring. Radiation Protection Dosimetry, 1(3), 227-232.

Sellin, P. J. (2003). Recent advances in compound semiconductor radiation detectors. Nuclear Instruments and Methods in Physics Research Section A: Accelerators, Spectrometers, Detectors and Associated Equipment, 513(1-2), 332-339.

Sherr, R. (1947). Scintillation Counter for the Detection of a-Particles. Review of Scientific Instruments, 18(10), 767-770.

Sikkema, C. P. (1970). A parallel plate ionization chamber with gas multiplication. Nuclear Instruments and Methods, 81(1), 189-194.

Trenn, T. J. (1986). The Geiger-Müller Counter of 1928. Annals of Science, 43(2), 111-135.

Underwood, E. A. (1945). Wilhelm Conrad Röntgen (1845-1923) and the early development of radiology. Proceedings of the Royal Society of Medicine, 38(12), 697-706.

Wibig, T., Kołodziejczak, K., Pierzyński, R., \& Sobczak, R. (2006). Educational studies of cosmic rays with a telescope of Geiger-Müller counters. Physics Education, 41(6), 542.

Zaitseva, N., Glenn, A., Carman, L., Hatarik, R., Hamel, S., Faust, M., ... \& Payne, S. (2011). Pulse shape discrimination in impure and mixed single-crystal organic scintillators. IEEE Transactions on Nuclear Science, 58(6), 3411-3420. 


\title{
Optical Phase Locked Loop Advancement
}

\author{
Poushali Mallick, Pritam Mandal, Rahul Paul, Santanu Moi, Purnendu Prakas Pal ${ }^{\star}$ \\ Department of Physics, Surendranath College, Kolkata, India \\ *Corresponding Author's Email: purnenduprakaspal@gmail.com
}

\begin{abstract}
Understanding of phase lock theories requires knowledge of several disciplines of engineering mathematics, nonlinear mechanics, control theory, Fourier transform, and Laplace transform of functions. Since the exciting discovery of LASER in 1954, scientists have been trying to shift the operating frequency from microwave to optical region of the frequency spectrum. The investigation on Phase Locked Loop (PLL) was thus in progress from radio frequency to optical region. Technological development in laser sources and optical detectors and above all Optical Phase Locked Loop (OPLL) will find their wide applications in telecommunications, radar systems, signal processing, tracking missile systems and satellite communication. Some mechanism and development of PLL and OPLL in this area will be briefly discussed in this article.
\end{abstract}

\section{Keywords: Optical Phase Locked Loop; Phase Locked Loop; Voltage Controlled Oscillator; Voltage Controlled Laser Oscillator; Gunn Diode Oscillator; IMPATT Diode Oscillator; DPLL and FSK}

\section{INTRODUCTION}

Optical Phase Locked Loops are expected to have a wide range of applications in fiber optic communication networks. One such application is the reconstruction of carrier for homodyne receivers (Kazovsky \& Atlas, 1990). The OPLL performance is unlike that of an ordinary PLL - it depends on the various noises, and the loop propagation delay acts in a detrimental way (Hodgkinson, 1985). The loop propagation delay is inevitable in practical situation and is not negligible for the system where the loop delay time is more than bit duration (Salz, 1985). Large number of works has been done on linearized OPLL analysis considering the impact of laser phase noise and detector shot noise. The basic limitations in designing OPLL are these noise sources, particularly the phase noise of the optical sources that introduce a harmful effect in the loop. The loop delay time imposes a stringent laser line width requirement for FSK homodyne detection.

\section{LITERATURE REVIEW}

The OPLL system using nonlinear analysis which can accommodate larger loop delay extending the loop stability and the commercially available laser sources may be employed with the better loop performance than the existing system is also described.

\section{About Phase Locked Loop (PLL)}

The origin of the phase-lock technique Gardner (1966) dates to the time of Huygens (1626-1695), who was credited with the discovery of the 'synchronization phenomenon' or 'phase locking phenomenon' as it is now-a-days called. However, the significance of synchronization of two clocks of Huygens' discovery could not be appreciated till the dawn of the twentieth century when Lord Rayleigh (1894), Appleton (1922) and van der Pol (1922) rediscovered the phenomenon in electronic circuits. At this stage, the birth of the most elemental form of the phase-locked loop took place and it is called the Injection Synchronized Oscillator (ISO). The recent improved version of the ISO is the so-called PhaseLocked Loop (PLL). After this large numbers of papers were published by scientists and engineers from all over the globe, and soon it was found that the phase locked loop can be used to build the most narrow-band, sensitive and flexible receivers of the world. As a result of these studies a very wide zone 
of application areas of PLL was identified, of which mention may be made of the following:

1. The phase locked loops are used in ranging devices and radar systems for tracking artificial satellites and planets.

2. Noisy output of ordinary oscillators can be cleaned up with the help of phase lock techniques.

3. A phase-locked loop can be used as a frequency demodulator.

4. Dynamic measurement of physical constants, like dielectric constant, can be measured with the help of PLL.

Because of increasing emphasis on digital circuitry, research works have been done on Digital PhaseLocked Loop (DPLL) and the response characteristics and stability (Biswas, 1989; Hagiwara \& Nakagawa, 1986) studied.

\section{Mechanization of the Phase Locked Loop}

The simplest form of a phase locked loop is shown in figure 1 (Biswas, 1988). It consists of a phase sensitive detector, a low pass filter and a voltage-controlled oscillator. The phase sensitive detector is a multiplicative device, the purpose of which is to produce an output voltage as a function of the instantaneous phase difference between the reference input and the output of the voltage-controlled oscillator (VCO). Figure 1: The Block Diagram of a Phase Locked Loop (PLL)

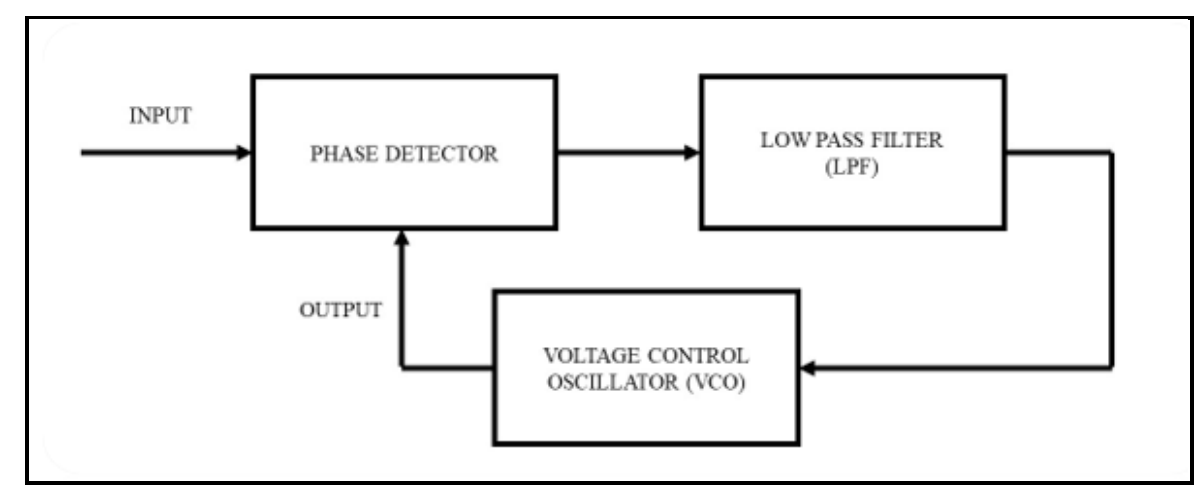

Figure 1: The Block Diagram of a Phase Locked Loop (PLL)

Atypical phase detector is shown in figure 2. The output of the phase detector is given by

$$
\mathrm{Vo}=2 \mathrm{~V} 1 \operatorname{Sin} \varphi
$$

The VCO is an oscillator, the frequency of which can be modulated in accordance with an input voltage. Thus, phase locked loop is an electronic feedback-controlled device. It operates in the following way. To begin with, let us assume that the frequency of the reference input is equal to the center frequency of the VCO. Therefore, the output of the phase detector that depends upon the phase or the instantaneous frequency difference between the two signals will be zero. Now if the frequency of the VCO tries to drift, this change in frequency will be first felt by the phase detector as a phase difference, and it will produce a voltage in correspondence to a measure of the phase difference. This voltage will correct the frequency of the voltage-controlled oscillator in such a way as to reduce the error in frequency. Similarly, if the frequency of the reference input changes by certain amount, then the output of the phase detector will shift the frequency of the VCO by the same amount. At this point it is to be noted that there is a limit to the frequency difference between the input and the output of the VCO up to which the frequency of the local oscillator can be controlled. 


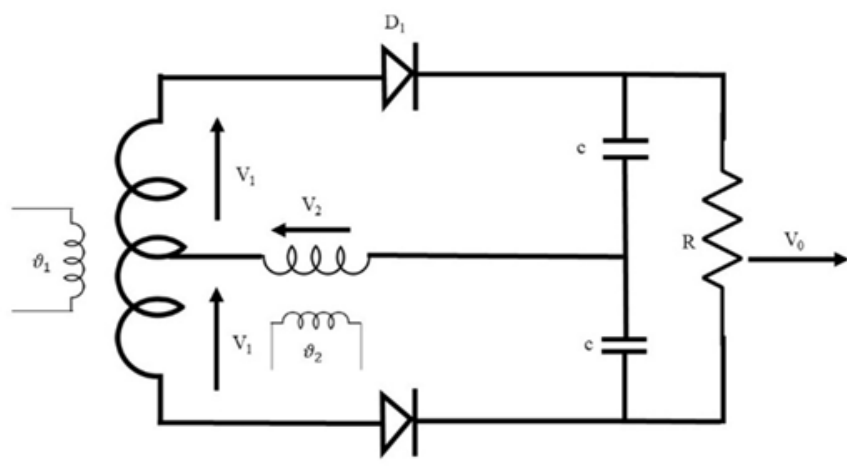

Figure 2: A Typical Phase Detector

\section{Figure 2: A Typical Phase Detector}

The output of the phase detector is connected to the voltage-controlled oscillator through a low pass filter. It modifies the response characteristics of the PLL.

\section{DISCUSSION}

\section{Further applications of Phase-Lock Principle}

Phase Locked Loops find many applications in the field of science and technology, such as, physics, biophysics, biology, telecommunications, electrical engineering, etc. Phase lock principles are utilized in signal processing at radio frequency as well as higher frequencies. At microwave and millimeter wave regions the principle of injection synchronization is commonly used because of the simplicity of circuit arrangement (Cullen \& Forrest, 1974; Biswas et al. 1981; Biswas et al. 1983; Kuno, 1973; Daikoku, \& Mizushima, 1971; Kurokawa, 1973; Gustafsson, Lundstrom \& Hansson, 1973; Hines, 1970; Nakajima \& Ikenouse, 1978; Takayama, 1972). In microwave and millimeter wave regions of the spectrum, mostly solid-state oscillating devices like Gunn diode, IMPATT, etc. are used now-a-days. The synchronization characteristics of microwave solid state oscillators are somewhat different from those of the oscillators operating at radio frequencies. The differences are mainly because of two reasons (Cullen \& Forrest, 1974) microwave solid state oscillators are modeled as nonlinear admittance functions rather than as a nonlinear conductance function, like in a van der Pol oscillator and (Biswas, 1988) aside their nonlinear dependence on the amplitude of the device current or voltage, they are nonlinear functions of the frequency of the oscillating current or voltage. As a result, the locking characteristic of a synchronized oscillator becomes asymmetric in nature on the two sides of the center frequency.

For certain applications, synchronization of microwave IMPATT oscillator and transistor oscillator modulated by laser beams (Yen \& Barnoski, 1978; Forrest \& Seeds, 1978) has also been proposed and many papers were published in this field.

\section{Locking Range}

Synchronization of an oscillator is nothing but a forced vibration. The locking range i.e., the maximum frequency error up to which locking is maintained (Biswas, 1988) is given by

$$
\Omega=K(\operatorname{Sin} \varphi / a)
$$

Where 'a' is the normalized amplitude of the oscillator, ' $\varphi$ ' is the phase difference and ' $K$ ' is related with center frequency of the oscillator.

\section{Optical Phase Locked Loop}

Several works on PLL and synchronized solid-state oscillators in radio frequency, microwave and mm 
wave had been done. Now-a-days scientists have shifted the frequency to optical region (Biswas, 1988; Gardner, 1966; Yen \& Barnoski, 1978; Forrest \& Seeds, 1978; Hodgkinson, 1985) of the frequency spectrum after discovery of solid-state laser sources. A simple block schematic of an Optical Phase Locked Loop (OPLL) is shown in figure 3. The different loop components are photo detector, loop filter, Voltage Controlled Laser Oscillator (VCLO) and an optical phase detector. The phase modulator possesses linear phase modulation characteristic, and it modulates the phase of the VCLO output. A double balanced phase detector comprising of photo detectors has been used, which does not need any low pass filter to reject the sum frequency components, and an optical phase modulator is used to compensate the loop delay. In their analysis, effect of shot and phase noise with loop propagation delay has been included.

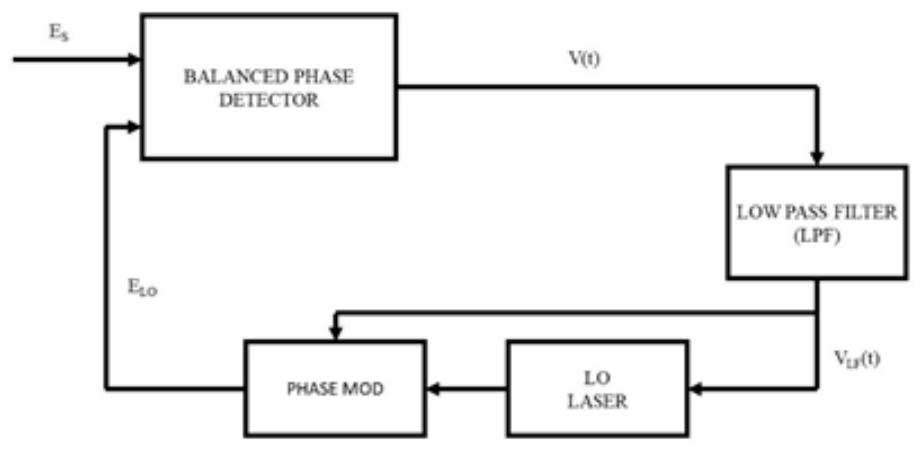

Figure 3: Block Diagram of Optical Phase Locked Loop (OPLL)

$$
\begin{aligned}
& E_{S}=\sqrt{2 P_{S}} \sin \left[\omega_{\mathrm{s}} \mathrm{t}+\varphi_{\mathrm{s}}(\mathrm{t})\right] \ldots \ldots \ldots \ldots \ldots \ldots \ldots \ldots \ldots \\
& \mathrm{E}_{\mathrm{L}}=\sqrt{2 P_{L O}} \operatorname{Cos}\left[\omega_{\mathrm{o}} \mathrm{t}+\varphi_{\mathrm{L}}(\mathrm{t})+\theta(\mathrm{t})+\psi(\mathrm{t})\right]
\end{aligned}
$$

Where Ps and PLo are the source and Local Oscillator (LO) laser power respectively, $\omega_{\mathrm{s}}$ and $\omega_{\mathrm{o}}$ are the frequencies of the source and LO laser, $\varphi_{s}(t)$ and $\varphi_{L}(t)$ are the phase noise contribution of source and LO laser, $\theta(t)$ is the phase part due to feedbac $k$ control of the loop and $\psi(t)$ is phase modulation due to phase modulator.

Now, $\theta(\mathrm{t})$ and $\psi(\mathrm{t})$ are expressed as:

$$
\begin{aligned}
& \Theta(\mathrm{t})=2 \pi \mathrm{K} L O \int_{-\infty}^{t} V_{L F}\left(T-\tau_{D}\right) d T \\
& \Psi(\mathrm{t})=\mathrm{K}_{\mathrm{PM} V_{\mathrm{LF}}(\mathrm{t}-\mathrm{TD}) \ldots \ldots \ldots \ldots \ldots \ldots \ldots \ldots}
\end{aligned}
$$

Where $\mathrm{TD}_{\mathrm{D}}=$ loop delay time, $\mathrm{K}_{\mathrm{LO}}=\mathrm{LO}$ laser sensitivity, $\mathrm{K}_{\mathrm{PM}}=$ phase modulator sensitivity and $\mathrm{V}_{\mathrm{LF}}$ is the low pass filter output.

The power spectral densities of laser phase noise and shot noise are given by,

$$
\mathrm{S}_{\mathrm{PH}}(\mathrm{f})=\delta \mathrm{v} /\left(\pi^{2}\right) \quad \mathrm{rad} / \mathrm{Hz}
$$

where,

$$
\begin{aligned}
& \delta v=\text { sum of the spectral line width of the laser, } \\
& f=\text { detected optical frequency, }
\end{aligned}
$$

and

$$
S_{S N}(f)=2 e R\left(P_{S}+P_{L o}\right) r^{2} \quad V^{2} / r a d
$$

where, $\mathrm{R}$ is photo detector responsivity, ' $e$ ' is the electronic charge, ' $r$ ' is the transimpedance of the detector. 
As per observation of the computer simulation results, the above OPLL showed better performance than the conventional loop.

\section{CONCLUSION}

Phase Locked Loop (PLL) and Optical Phase locked Loop (OPLL) have revolutionized the field of electronic communications and optical communications and are the backbone of today's communication networks. Apart from communications, PLL and OPLL are also expected to find wide applications in the field of biological science, remote sensing, and radar systems. After discovery of solid-state devices like Gunn diode oscillator and IMPATT diode oscillator in the microwave and mmwave region, synchronization techniques have been utilized in the receivers of communication systems. Frequency Modulated signal are detected with the help of PLL, OPLL and synchronized oscillators. The use of microwave techniques in light wave system through OPLL is essential in recent communication systems. With further developments of the technology involved in OPLL, the field of communication is expected to grow significantly in the coming years with new applications.

\section{ACKNOWLEDGEMENT}

The authors express their sincere thanks to Department of Biotechnology, Government of India for the funding from DBT-STAR College grant, under which this review project was conducted. They are also grateful to the Principal and the DBT-STAR Coordinator, Surendranath College, for their support and encouragement in implementing this review project at undergraduate level.

\section{REFERENCES}

Biswas, B. N. (1988). Phase lock theories and applications. Oxford \& IBH Publishing Company.

Biswas, B. N., Bhattacharya, A. K., Pal, P., Mondal, D., Ray, S. K., \& De, R. (1989). New softwarecontrolled digital phase-locked loop. Electronics Letters, 25(16), 1097-1098.

Biswas, B. N., Ray, S. K., Pramanik, K., Sadhu, M., \& Bandyopadhyay, D. (1983). Hold-in characteristics of an extended range Gunn oscillator system. IEEE Transactions on Microwave Theory and Techniques, 31(3), 271-276.

Biswas, B. N., Ray, S. K., Pramanik, K., Sadhu, M., \& Bhattacharya, A. K. (1981). Modified circuit theoretic approach on synchronized microwave IMPATT oscillators. INDIAN JOURNAL OF PURE \& APPLIED PHYSICS, 19(5), 464-471.

Cullen, A. L., \& Forrest, J. R. (1974, December). Analytic theory of the IMPATT diode and its application to calculations of oscillator locking characteristics. In Proceedings of the Institution of Electrical Engineers (Vol. 121, No. 12, pp. 1467-1474). IET Digital Library.

Daikoku, K., \& Mizushima, Y. (1971). Properties of injection locking in the non-linear oscillator. International journal of electronics, 31(3), $279-292$.

Forrest, J. R., \& Seeds, A. J. (1978). Optical injection locking of impatt oscillators. Electronics Letters, 14(19), 626-627.

Gardner, F. M. (1966). Phase-Lock Techniques. Wiley. New York.

Gustafsson, L., Lundstrom, K. I., \& Hansson, G. H. B. (1973). Maximum Phase-Locking Bandwidth Obtainable by Injection Locking (Short Papers). IEEE Transactions on Microwave Theory and Techniques, 21(5), 353-355.

Hagiwara, M., \& Nakagawa, M. (1986). DSP-type first-order digital phase-locked loop using linear phase detector. Electronics and Communications in Japan (Part I: Communications), 69(6), 99-107. 
Hines, M. E. (1970). Negative-resistance diode power amplification. IEEE Transactions on Electron Devices, 17(1), 1-8.

Hodgkinson, T. G. (1985). Phase-locked-loop analysis for pilot carrier coherent optical receivers. Electronics Letters, 21(25), 1202-1203.

Kazovsky, L. G., \& Atlas, D. A. (1990). A 1320-nm experimental optical phase-locked loop: performance investigation and PSK homodyne experiments at $140 \mathrm{Mb} / \mathrm{s}$ and $2 \mathrm{~Gb} / \mathrm{s}$. Journal of Lightwave Technology, 8(9), 1414-1425.

Kuno, H. J. (1973). Analysis of nonlinear characteristics and transient response of IMPATT amplifiers. IEEE Transactions on Microwave Theory and Techniques, 21(11), 694-702.

Kurokawa, K. (1973). Injection locking of microwave solid-state oscillators. Proceedings of the IEEE, 61(10), 1386-1410.

Nakajima, M., \& Ikenouse, J. (1978). Locking phenomenon in Microwave Oscillator Circuits. International Journal of Electronics, 44(5), pp 465-472.

Salz, J. (1985). Coherent lightwave communications. AT\&T technical journal, 64(10), 2153-2209.

Takayama, Y. O. I. C. H. I. R. O. (1972). Dynamic behavior of nonlinear power amplifiers in stable and injection-locked modes. IEEE Transactions on Microwave Theory and Techniques, 20(9), 591-595.

Yen, H. W., \& Barnoski, M. K. (1978). Optical injection locking and switching of transistor oscillators. Applied Physics Letters, 32(3), 182-184. 


\title{
Coulomb Blockade Effect in the Context of Set
}

\author{
Rajibul Islam, Rajdeep Saha, Snehasis Mondal, Ranjit Maity* \\ Department of Physics, Surendranath College, Kolkata \\ ${ }^{*}$ Corresponding Author's Email: ranjit_maity@yahoo.co.in
}

\begin{abstract}
As we scale materials down to nanoscale, some interesting properties and phenomenon start to emerge that are not so obvious at macroscale, and sometimes completely different from its bulk properties. One such phenomenon is Coulomb Blockade (CB) which arises due to, in layman terms, Coulomb repulsion. As we make a device smaller, its capacitance decreases and in turn its charging energy, which is inversely proportional to its capacitance, increases. When the device is very small, the charging energy becomes measurably large enough to even impede the tunnelling of electron. Single Electron Transistor (SET) which can control the transport of only one electron is based on this principle. This review article is intended to explore the principle of Coulomb blockade in context of devices like SETs and underlining the promising application and limitation of implementation of such devices.
\end{abstract}

Keywords: Sets; Qd's; Nanoelectronics; Coulomb Blockade

\section{INTRODUCTION}

Concept of miniaturisation of devices is not a new concept. It dates to the year 1959, when physicist Richard Feynman, in his lecture "There's Plenty of Room at the Bottom: An Invitation to Enter a New Field of Physics" at the annual meeting of American Physical Society on 29th December 1959 (Taylor, 1960 ), he brought up the idea of miniaturisation and direct manipulation of matter at atomic and molecular levels.

It was after three decades that Feynman's foresight became reality with the discovery of carbon nanotube by Sumio ljima in 1991 at the NEC laboratory, Tsukuba, Japan (Drexler, 1992). Since then, various ways to synthesize materials in the size of nanometer had been researched and finally led to the development of quantum dots (QD). This $\mathrm{QD}$ is a nanoparticle having all the dimensions reduced to below $100 \mathrm{~nm}$, which is small enough to be used in observing Coulomb blockade (CB), and it is also the main building block of SET.

\section{LITERATURE REVIEW}

\section{Coulomb Blockade Effect}

Single-electron transistors (SETs) are the simplest device which follows the principle of coulomb blockade.

The device has two electrodes, the "drain" and the "source". They are joined by tunnel junction to a single common electrode, the "island", which has a very low self-capacitance (refer to figure 1). The electric potential of the island can be controlled by a third electrode known as the "gate."

When a device (island) gets tiny enough, in the nanoscale range, its capacitance, which is directly proportional to its diameter, becomes noticeably low. This low capacitance raises the charging energy of the island and prevents electron transmission. This suppression of electron transfer is known as the Coulomb Blockade (Wasshuber, 1997). 


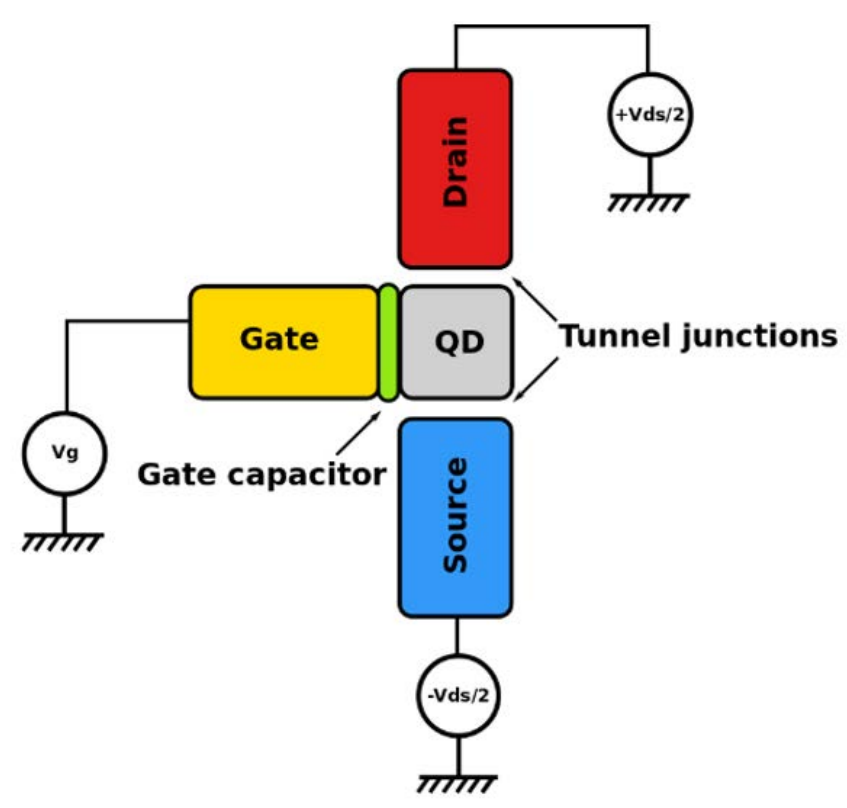

Figure 1: Coulomb Blockade Effect

When a single electron is added to the island of charge $\mathrm{Q}$, the change in electrostatic energy is given by,

$$
\Delta E=\frac{e Q}{C}
$$

The corresponding change in potential is given by,

$$
\Delta V=\frac{e}{C}
$$

\section{Conditions to observe Coulomb blockade effect}

1. To detect Coulomb blockade effects, the change in electric energy $\Delta \boldsymbol{E}$ must be substantially greater than the change in thermal energy $\boldsymbol{K}_{\boldsymbol{B}} \boldsymbol{T}$ at the working temperature. Otherwise, electrons can tunnel through the connections at random without being stopped by the Coulomb energy. Therefore,

$$
C \ll \frac{e^{2}}{K_{B} T}
$$

2. The random variation of the number of electrons in the dot (island) causes fluctuations in potential energy of the order of $\frac{e^{2}}{c}$. Therefore, we should have

$$
R_{t}>\frac{h}{e^{2}} \sim 25.8 k \Omega
$$

where $\mathbf{R}_{\mathbf{t}}$ is the tunneling resistance 


\section{DISCUSSION}

\section{Applications of SET:}

1. The SET are charge sensors that can read out the spin or charge q-bits that are confined in QDs. For both dc and radio frequency single shot measurements, the SET can be used as an effective signal-to-noise ratio probe (Kumar \& Dubey, 2013).

2. Because of their high sensitivity, SETs have been used as supersensitive electrometers in a variety of physical measurements.

3. Because the SET can detect infrared signals at room temperature, it can be used as an IR sensor in sophisticated devices such as IR cameras.

\section{Limitations of SET:}

Despite the wide range of applications, the device's implementation of SET has some limitations, as follows:

1. The requirement $\mathrm{EC} \sim 100 \mathrm{kBT}$, which in practice means sub-nanometer island size for room temperature operation, is a major limitation of all known types of single electron logic devices.

2. Another limitation, given the size of the SET, is connecting it to the outside world via normal wire connections.

3. The randomness of the background charge is another major limitation of single electron logic circuits (Kumar \& Kaur, 2010).

\section{CONCLUSION}

The developing importance of the Internet of Things and healthcare programs has a greater impact on the electricity intake of virtual devices. Ultra-low electricity intake is one of the essential research topics withinside the electronics world for this purpose. The brilliant wide variety of tiny computer systems utilized in each day life (for example, cellular telephones and domestic electronics) necessitates an excessive degree of energy consumption from the carried-out devices. In this case, the SET seems to be an excellent candidate for attaining this low energy variety even as keeping an excessive degree of tool integration.

\section{ACKNOWLEDGEMENT}

The authors express their sincere thanks to Department of Biotechnology, Government of India for the funding from DBT-STAR College grant, under which this review project was conducted. We are also grateful to the Principal and the DBT-STAR Coordinator, Surendranath College, for their support and encouragement in implementing this review project at undergraduate level.

\section{REFERENCE}

Drexler, K. E. (1992). Nanosystems: Molecular Machinery, Manufacturing, and Computation. Wiley

Kumar, A., \& Dubey, D. (2013). Single electron transistor: Applications and limitations. Advance in Electronic and Electric Engineering, 3(1), 57-62.

Kumar, O., \& Kaur, M. (2010). Single electron transistor: Applications \& problems. Int J VLSI Des Commun Syst (VLSICS), 1(4), 24-29.

Taylor, L. (1960). Student Life: The Caltech Y. Engineering and Science, 23(5), 17-19.

Wasshuber, C. (1997). About single-electron devices and circuits. 


\title{
Electromagnetically Induced Transparency in Coherent Atomic Media
}

\author{
Arin Bhattacharya, Abhasita Das Munshi, Abhiroop Ghosh, Anand Singh, Aparajita Das* \\ Department of Physics, Surendranath College, Kolkata, India \\ ${ }^{*}$ Corresponding Author's Email: apdsnc@gmail.com
}

\begin{abstract}
Quantum interference occurs between the optical transition pathways when the quantum states of atoms or molecules are coherently prepared by lasers. It can dramatically modify the optical responses of an atomic medium to the weak probe field in presence of a strong pump field. A beautiful manifestation of the quantum interference or more specifically destructive quantum interference is the electromagnetically induced transparency (EIT). In this article the authors have discussed the basic theory of EIT by considering the atomic dynamics. The responses of two-level and three-level atomic systems to the probe field are presented here by examining the probe absorption and dispersion profiles. Some potential applications of EIT are also mentioned here.
\end{abstract}

Keywords: Electromagnetically Induced Transparency; Absorption; Transparency; Optical Bloch Equations

\section{INTRODUCTION}

Electromagnetically induced transparency (EIT) in atomic medium is the phenomenon where an initially absorbing atomic medium becomes transparent to a weak probe field (typically lasers) in the presence of a strong control field (laser field). EIT receives special interest since it can be described by quantum interference induced by the coherence effect. In this article the authors have studied the underlying physics behind the occurrence of EIT in the three simplest atomic configurations with three atomic states (three level systems) i.e., $\Lambda, \Xi, \vee$-type systems under the semi-classical approach. Firstly, they have presented the basic light-atom interaction phenomenon in two level system. Then the optical Bloch equations (OBEs) are constructed for stationary atoms under the framework of density matrix. For each three level systems ( $\Lambda, \equiv, \vee$-type systems), OBEs are solved numerically to have the corresponding coherence term that are directly proportional to the probe absorption in the medium. The probe absorption and dispersion profiles are represented as the variations of probe absorption, dispersion as a function of probe detuning. Finally, they have summarized our discussion and have mentioned some works based on EIT technique.

\section{LITERATURE REVIEW}

EIT was first proposed theoretically by Jacob Khanin and Olga Kocharovskaya. Later, it was experimentally demonstrated by Boller, Imamoğlu \& Harris in 1991. The transparency in the atomic medium, induced by the electromagnetic field (EM field) is the outcome of a destructive interference between different atomic excitation or transition pathways. Over decades, EIT has made possible many interesting and important progresses to manipulate atomic-optical responses. For examples, a light pulse is slowed down significantly or even trapped in an atomic medium, lasing is observed without population inversion, atoms are laser cooled below the recoil limit etc. The name EIT was first referred by Harris, Field \& Imamoğlu (1990). In their paper the resonantly enhanced non-linear susceptibility based on EIT was proposed. The first experimental demonstration of EIT was followed in 1991 again by the same Group led by Boller, Imamoğlu \& Harris (1991). Their experiment was carried out in a lambda $(\Lambda)$ scheme in Strontium vapour using pulsed lasers. EIT on a collisionally broadened resonance transition of $\mathrm{Pb}$ vapour was reported by Field, Hahn \& Harris (1991). They anticipated the applications of EIT in non-linear optical processes for the first time. Afterward, experimental observation of 
transparency on a transition in the blue spectral region, induced by a continuous wave infrared coupling field in a Doppler broadened medium was presented by Boon et al. (1998). This experimental result was supported by extensive theoretical modelling of the system in a V-type scheme in atomic rubidium vapour. In principle it is possible to achieve a large enhancement of index of refraction via quantum interference effects and EIT is one of them. Eberly, Pons \& Haq (1994) in University of Rochester reported first observation of spatial evolution of dressed field pulses, the exact analogue of temporal evolution of dressed atom. Their result provided the information of EIT propagation. A theoretical and experimental investigation into the viability of $\Lambda, \Xi, V$ systems within $R b$ atom for observation of EIT was done by Fulton et al. (1995). Except the investigation of optical response, the spectral properties are also examined by the EIT technique. The hyperfine structures of highly excited atomic states were determined by EIT experiment based on atomic coherence by Jin, Li \& Xiao (1995). Kasapi (1996) applied EIT to isotope discrimination by adjusting the intensity of a coupling laser. One isotope was made resonantly opaque while another was rendered transparent to a probe. With the gradual theoretical and experimental advances since 1990, a revolutionary observation came forward in the year 1999. Danish physicist team led by Hau et al. (1999) from Harvard University and the Rowland Institute for Science reported an experimental demonstration of EIT in an ultra-cold gas of sodium atoms, in which the optical pulses propagated at twenty million times slower than the speed of light in vacuum. The gas was cooled to nano kelvin temperatures by laser and evaporative cooling. The quantum interference controlling the optical properties of the medium was set up by a 'coupling' laser beam propagating at a right angle to the pulsed 'probe' beam. In conjunction with the high atomic density, they succeeded in slowing a beam of light to about 17 meters per second using EIT technique.

\section{DISCUSSION}

In classical theory of radiative emission and absorption, the atoms are regarded as oscillating dipole. When an atom interacts with EM wave of angular frequency $\omega$, it may be modeled as an EM field driven oscillator system.

\section{(A) Two Level System}

Atoms have many quantum levels and many atomic transitions between them are possible. If the frequency of incident EM wave coincides with one of the optical transition frequencies, all other levels can be excluded except the two levels of transition. Thus, many level system is reduced into a two-level system as shown in figure 1. To understand the resonant light-atom interaction, it is convenient to adopt a semi-classical approach in which the atoms are treated as quantum object, but the light is treated as classical. The link between the classical and quantum theories lies in the transition dipole moment (Dba) corresponding to the transition between two states $\mid \psi a>$ and $\mid \psi b>$. Dba is given as,

$$
\mathrm{Dba}=-\mathrm{e}<\psi \mathrm{b}|\mathrm{r}| \psi \mathrm{a}>
$$

where, a corresponds to lower energy level and b corresponds to the higher energy level. $\mathrm{e}$ is the electronic charge.

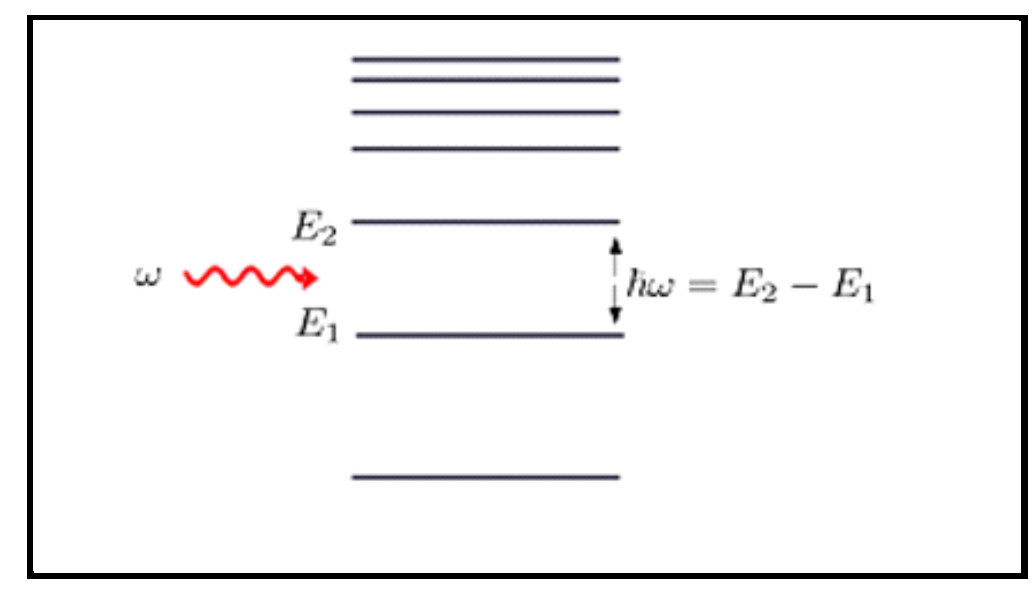

Figure 1: Schematic Diagram of Two-Level System 


\section{Rabi Frequency}

For an atom with two energy levels with energies E1 and E2 (For a two-level system) in the presence of EM field of frequency $\omega$, let us assume that $\omega=\omega 0+\delta \omega$ (i.e., very close to resonance with the transition). Now, the light-atom interaction Hamiltonian can be written as,

$$
\mathrm{H}=\mathrm{HO}+\mathrm{H}^{\prime}
$$

Under Semi-Classical approach the perturbing term (light-atom interaction term) $\mathrm{H}^{\prime}$ is given by,

$$
H^{\prime}=- \text { e r.E }
$$

If they choose that light is polarized along $x$ axis then,

$$
E=E 0 \cos \omega t x
$$

So, H' becomes,

$$
\begin{aligned}
\mathbf{H}^{\prime} & =\frac{e E 0}{2}\left(\mathrm{e}^{\mathrm{i} \omega \mathrm{t}}+\mathrm{e}^{-\mathrm{i} \omega \mathrm{t}}\right)<\mathrm{i}|\mathrm{x}| \mathrm{k}> \\
& =-\frac{E 0}{2}\left(\mathrm{e}^{\mathrm{i} \omega \mathrm{t}}+\mathrm{e}^{-\mathrm{i} \omega \mathrm{t}}\right) \mu_{\mathrm{jk}} \\
& =\frac{\mathrm{h} \Omega \mathrm{R}}{4 \pi}\left(\mathrm{e}^{\mathrm{i} \omega \mathrm{t}}+\mathrm{e}^{-\mathrm{i} \omega \mathrm{t}}\right)
\end{aligned}
$$

The term $\Omega_{\mathrm{R}}=\left|\frac{2 \pi E 0 \mu j k}{h}\right|$ is called "Rabi frequency". Here, $\mu_{\mathrm{jk}}$ is the Dipole Matrix element.

\section{Introduction of density matrix}

For a two-level system the superposition state $|\psi\rangle$ can be written as,

$$
\left|\psi>=\mathrm{c}_{1}\right| 1>+\mathrm{c}_{2} \mid 2>
$$

$\mid \psi>$ is a pure state. Let us now introduce Density Operator $\boldsymbol{\rho}$ as,

$$
\boldsymbol{\rho}=|\psi><\psi|
$$

In general,

$$
|\psi><\psi|=\sum_{i, j=1,2}|i><i| \psi><\psi|j><j|
$$

It is obvious that the diagonal terms give the probabilities of being in the energy (upper and lower) states or the population of the states. The off-diagonal terms determine the atomic polarization. If $|\psi\rangle$ is a coherent superposition of states $|1\rangle$ and $|2\rangle$, then the off-diagonal terms reflect the phase dependence.|

\section{Advantages of density matrix}

1. A pure state can be described by a density operator as well as by a state vector. Both descriptions

are equivalent.

2. Two state vectors $\mid \psi>$ and $\mathrm{e}_{m}^{\mathrm{i} \theta}|\psi\rangle$ describe the same quantum state, though there exists an arbitrary phase factor for the state vectors. However, the state vectors correspond to same density operator. By using density operators, one can eliminates the phase. 
3. Expectation value of any observable $\mathbf{A}$ is

$$
<\mathbf{A}>=\frac{\operatorname{Tr}\{\rho A\}}{\operatorname{Tr}\{\rho\}}
$$

4. One can easily add damping or decoherence terms phenomenologically in this description.

\section{Time evolution of density operator and Optical Bloch Equation (OBE)}

The equation of motion of the density operator is given by,

$$
\begin{aligned}
\frac{d \rho}{d t} & =-\frac{2 \pi i}{h}[\mathbf{H}, \boldsymbol{\rho}] \\
& =-\frac{2 \pi i}{h}[\mathbf{H} \boldsymbol{\rho}-\boldsymbol{\rho} \mathbf{H}]
\end{aligned}
$$

This equation is like the classical Liouville's equation. For a two-level system the time evolution of the density operators takes the following forms,

$$
\begin{aligned}
& \dot{\hat{r}}_{11}=\frac{i \Omega}{2}\left[\rho_{12} \mathrm{e}^{-\mathrm{i} \omega \mathrm{t}}-\mathrm{c} . \mathrm{c}\right] \\
& \dot{\rho}_{22}=\frac{i \Omega}{2}\left[\rho_{21} \mathrm{e}^{\mathrm{i} \omega \mathrm{t}}-\mathrm{c} . \mathrm{c}\right] \\
& \left.\dot{\rho}_{12}=\frac{i \Omega}{2}\left(\rho_{11}-\rho_{22}\right) \mathrm{e}^{\mathrm{i} \omega \mathrm{t}}+\mathrm{i} \rho_{12} \omega_{0}\right] \\
& \left.\dot{\rho}_{21}=\frac{i \Omega}{2}\left(\rho_{22} \rho_{11}\right) \mathrm{e}^{-\mathrm{i} \omega \mathrm{t}}-\mathrm{i} \rho_{21} \omega_{0}\right]
\end{aligned}
$$

\section{Slowly Varying Envelope Approximation (SVEA)}

Off-diagonal elements of the density matrix describe charge oscillations caused by the applied fields. Hence, the temporal evolution of their amplitudes provides transient behavior of an oscillation before reaching any steady-state amplitude. So, the general form of the solution is

$$
\rho_{i j}(t)=\sigma_{i j}(t) e^{i \omega t}
$$

This approximation is called "Slowly varying envelope approximation".

\section{Phenomenological introduction of damping terms}

In real physical atomic systems, the spontaneous emission, atom-atom collisions, atom-wall collisions always persist. By virtue of these population decay processes the population to the levels under consideration are either removed or added. This removal or addition of population can be incorporated in the OBEs by introducing the decay terms as damping terms. In figure 2 the scheme of adding decay terms in the OBEs is explained. 


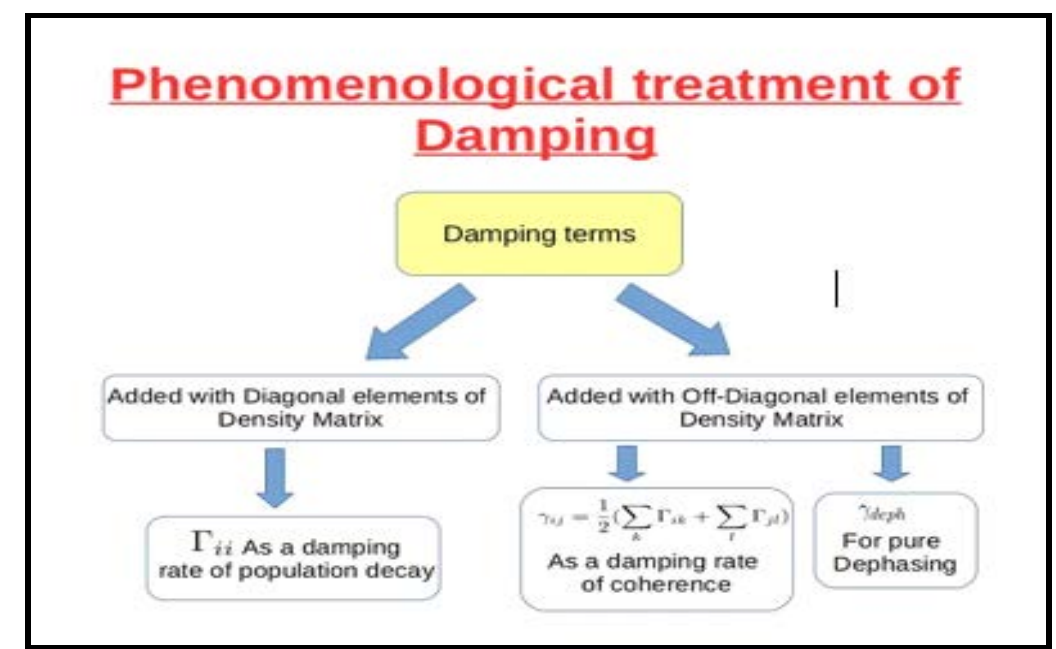

Figure 2: Phenomenological Treatment of Damping Terms

After using SVEA and adding damping terms, the OBEs for a two-level system become,

$$
\begin{aligned}
& \dot{\sigma}_{11}=\frac{i \Omega}{2}\left[\sigma_{12}-\mathrm{c} . \mathrm{c}\right]+\Gamma \sigma_{11} \\
& \dot{\sigma}_{22}=\frac{i \Omega}{2}\left[\sigma_{21}-\mathrm{c} . \mathrm{c}\right]-\Gamma \sigma_{11} \\
& \dot{\sigma}_{12}=\frac{i \Omega}{2}\left(\sigma_{11}-\sigma_{22}\right)-\left(v_{\perp}+\mathrm{i} \delta\right) \sigma_{12} \\
& \dot{\sigma}_{21}=\frac{i \Omega}{2}\left(\sigma_{22}-\sigma_{11}\right)-\left(v_{\perp}-\mathrm{i} \delta\right) \sigma_{21}
\end{aligned}
$$

Here, $v_{\perp}$ is given by, $v_{\perp}=\frac{\Gamma}{2}+v_{c}$.

$\delta$ is the probe detuning. $\Gamma$ is the rate of spontaneous decay, $v_{\mathrm{s}}$ is the additional rate describing pure dephasing (e.g., due to collisions of two-level atoms in a gas). Phenomenological treatment of damping terms is shown in figure 2 . Off diagonal and diagonal matrix elements of $\sigma$ give the coherence and the population of the system, respectively. For a three-level system one gets nine equations with nine variables. Similarly, for a four-level system they have sixteen equations, for five-level system they have twenty-five equations and so on.

\section{Steady state solution of $\mathrm{OBEs}$}

Now, each $\sigma_{i k}$ is divided into two parts i) real part $\left(\mathrm{u}_{i k}\right)$ defining dispersion and ii) imaginary part ( $\left.\mathrm{v}_{i k}\right)$ defining absorption. So, the susceptibility of the medium $(\gamma)$ related to $\sigma_{i k}$ is,

$$
\chi=-\frac{4 \pi}{h \in \Omega} \mathrm{N}_{0}\left|\mu_{\mathrm{ik}}\right|^{2}\left(\mathrm{u}_{\mathrm{ik}}+\mathrm{i} \mathrm{v}_{\mathrm{jk}}\right)
$$

Where, $\mathrm{N}_{0}, \epsilon_{0}, \mu_{\mathrm{k}}, \Omega$ are the atom density, permittivity of the medium, electric dipole matrix elements and probe Rabi frequency, respectively. Again, according to the conservation of population $\sum_{j} \rho_{\mathrm{ij}}=1$. So, $\sum_{j} u_{\mathrm{jj}}=1$ and eventually all $\mathrm{v}_{\mathrm{ii}}=0$. Now by substituting $\sigma_{\mathrm{ik}}$ by $\mathrm{u}_{\mathrm{ii}}$ and $\mathrm{v}_{\mathrm{ii}}$ for any $\mathrm{N}$-level system they have a set of equations, and they can be written in the following matrix form.

$$
\mathrm{AB}=\mathrm{C}
$$

Here, $\mathrm{A}$ is an $\mathrm{N} \times \mathrm{N}$ square matrix and $\mathrm{B}$ and $\mathrm{C}$ are $\mathrm{N} \times 1$ column matrices. From this they can get $\mathrm{B}$ as,

$$
\mathrm{B}=\mathrm{A}^{-1} \mathrm{C}
$$

The elements of B correspond to real and imaginary parts of different $\sigma_{j k}$ that provide absorption and dispersion of probe field connecting the atomic states. Similarly, all the features including the population can also be obtained.

In steady state i.e., $\dot{\sigma}_{\mathrm{jk}}=0(\mathrm{j}, \mathrm{k}=1,2)$, equations (6)-(9) are solved using the above-mentioned technique. The variation of probe absorption and dispersion $w . r . t$. the probe detuning $(\delta)$ for a two-level system is represented in figure 3 . 


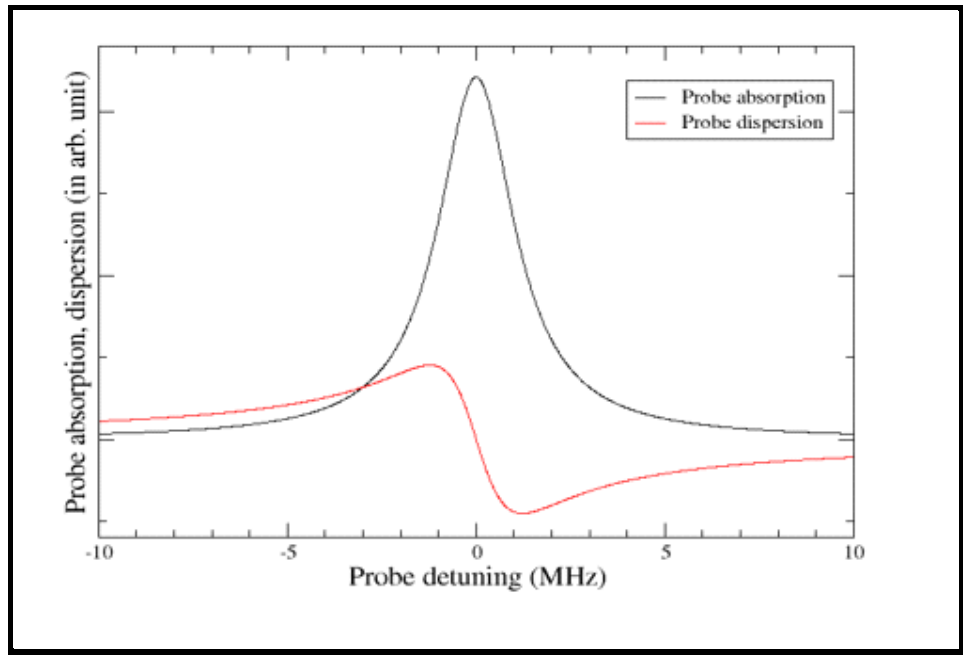

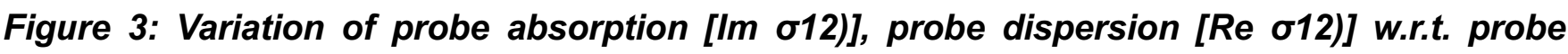
detuning (ס) for a two-level system

\section{Coherent superposition of state}

Wave-function of the two-level system can be written as a superposition of two states corresponding to the upper and lower energy level under consideration. So, $\left.\left|\psi>=c_{1}\right| 1\right\rangle+c_{2} \mid 2>$. $c_{1}$ and $c_{2}$ are the probability amplitudes. In case of statistical mixture, an atom can either be in state $\mid 1>0$ in state $\mid 2>$ at a certain point of time. But in case of light-atom interaction, light pulse links the phases of upper and lower level. If there is a definite phase relationship between $c_{1}$ and $c_{2}$, the wave function $|\psi\rangle$ becomes a coherent superposition state.

\section{(B) Three Level System}

\section{Various schemes}

A three-level system is a system with effectively two two-level systems. According as the shape of allowed transition lines in the schematic energy level diagram of a three-level system, three main choices of level scheme can be considered. In figure 4-6 three three-level systems are schematically illustrated using the hyperfine levels of ${ }^{87} \mathrm{Rb},{ }^{85} \mathrm{Rb},{ }^{11} \mathrm{Na}$ atoms, respectively.

$\Lambda$-type system

In a $\Lambda$-type system (figure 4) a coupling field couples the upper most state $\mid 2>$ (excited state) and the middle state $\mid 3>$ (ground state) whereas, a probe field connects the transition between the lower most state $\mid 1 \mathrm{i}$ (lowest ground state) and the upper most state $\mid 2>$ (excited state).

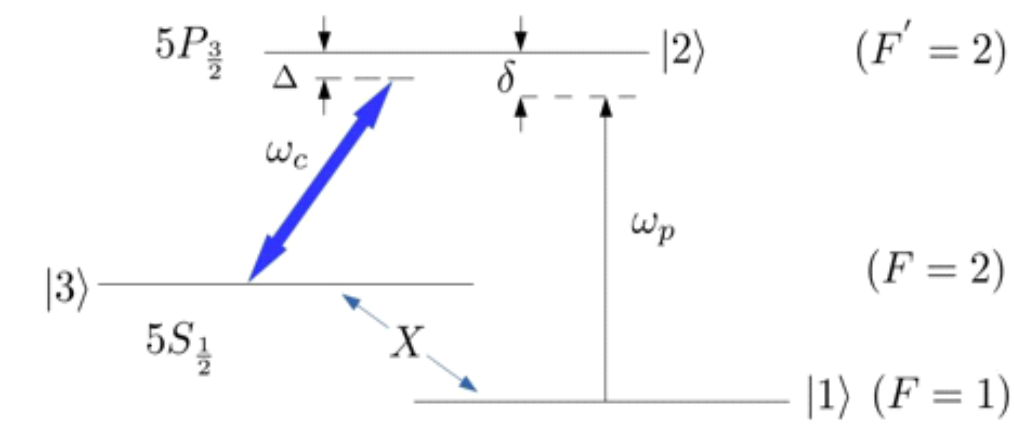

Figure 4: ^-type atom-laser coupling scheme (Hyperfine transitions of87Rb) 
三- type system:

三 systems (figure 5) are those for which coupling between two upper excited states $\mid 2>$ and $\mid 3>$ by the coupling field is considered. Here, the probe field is connected between the lowest state $\mid 1>$ (ground state) and the middle state $\mid 2>$.

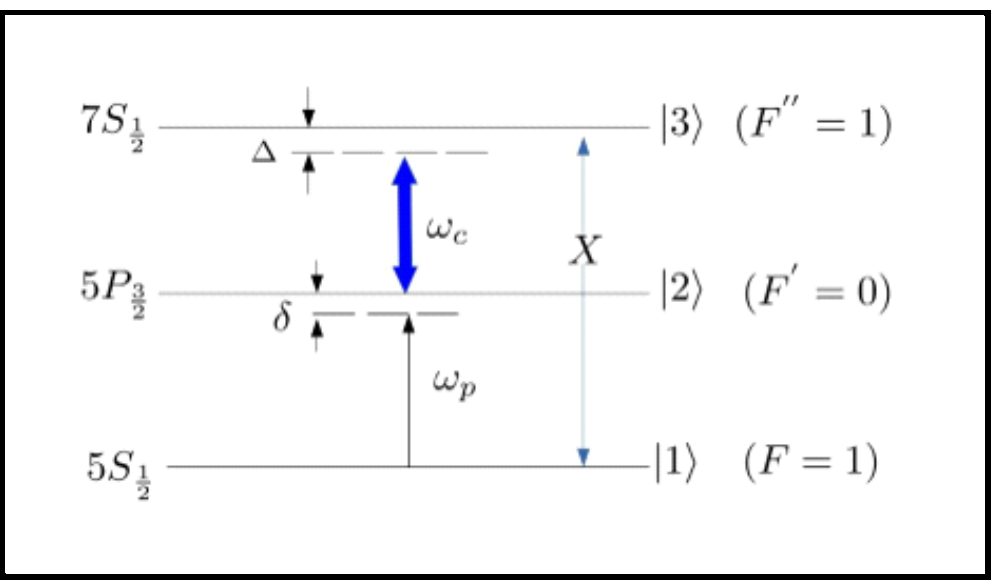

Figure 5: 三-type atom-laser coupling scheme (Hyperfine transitions of ${ }^{85} \mathrm{Rb}$ )

$\checkmark$-type system:

For a V-type system (figure 6) the lowest ground state |1>and the middle state $\mid 2>$ (one excited state) are coupled by the coupling field and the probe field connects the transition between the lowest ground state |1 >and the upper most (excited state) state $\mid 3>$.

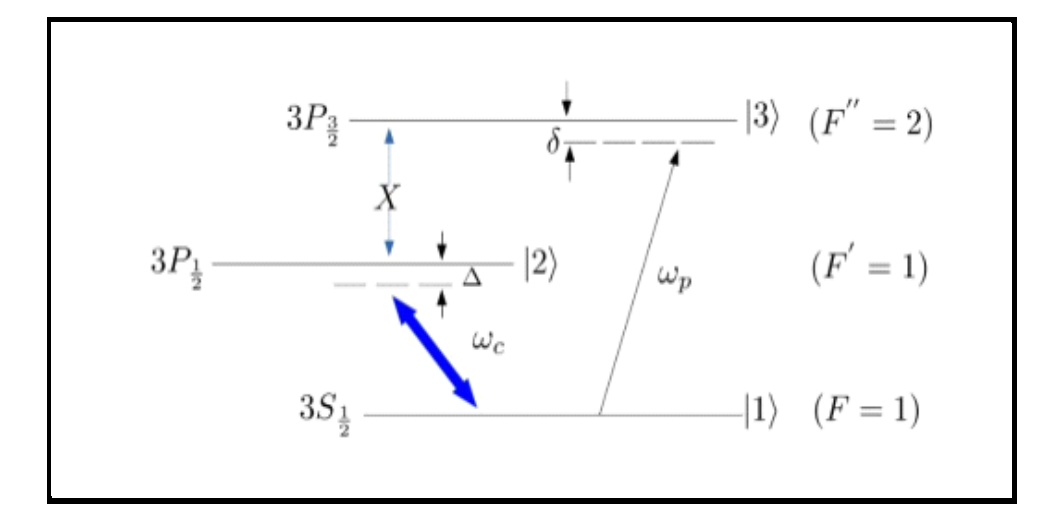

Figure 6: V-type atom-laser coupling scheme (Hyperfine transitions of ${ }^{11} \mathrm{Na} \mathrm{D}_{1}-\mathrm{D}_{2}$ ) Lines

\section{Concept of CPT and EIT}

Let us consider that the initial atomic states of a $\Lambda$-type system are prepared as a superposition of the lower two ground states. If now the system is illuminated by two strong $E \mathrm{M}$ fields, the probability of transition from one ground state to the excited state becomes zero. The transition between the other ground state and the excited state can still occur. As a result, to this, atomic population is trapped in one of the lower levels after some cycles of spontaneous decay from the excited state and transition to the excited state. Finally, no atom can go to the excited state even in the presence of the second field. Such state accumulated with the atomic population is called 'Dark state' and this phenomenon is called coherent population trapping (CPT). EIT can also be explained in the same way for a three-level atomlaser coupling scheme (Field, Hahn \& Harris, 1991; Fulton et al., 1995). For EIT, a weaker field is used as the probe field and a strong field is served as a pump field. Here, the atomic transition connected by the probe field stops and the system becomes transparent to the probe when dark state is present in the system. Along with the $\Lambda$-type system, EIT is possible to be observed in all the three systems mentioned above. In the following section they have discussed EIT in $\Lambda, \equiv$ and $V$-type systems by solving the OBEs in steady state for the three-level systems. 
Optical Bloch Equations for three level system

For a three-level system, the Optical Bloch Equations (OBE) can be obtained by the following formula.

$$
\dot{\rho}_{\mathrm{jk}}=-\sum \frac{2 \pi i}{h}\left[\mathrm{H}_{\mathrm{j} \rho} \rho_{\mathrm{lk}}-\rho_{\mathrm{jl}} H_{\mathrm{lk}}\right]-\text { decay matrix }
$$

Where, decay matrix contains decoherence terms $\mathrm{Y}_{\mathrm{jk}}=\frac{1}{2} \sum_{j<l} \Gamma_{\mathrm{jl}}+\frac{1}{2} \sum_{m<k} \Gamma_{\mathrm{km}}$

Steady state solutions of OBEs for $\Lambda$, $\equiv$ and $V$-type systems

After using Rotating Wave Approximation (RWA) and Slowly Varying Envelope Approximation (SVEA), one can get six coupled differential equations as the OBEs for the $\Lambda$, $\equiv$ and $V$-type systems. When the steady state is achieved, we obtain the solutions for the related density matrix elements for $\Lambda$, $\equiv$ and $\vee$ type systems by solving the OBEs of the three-level system numerically.

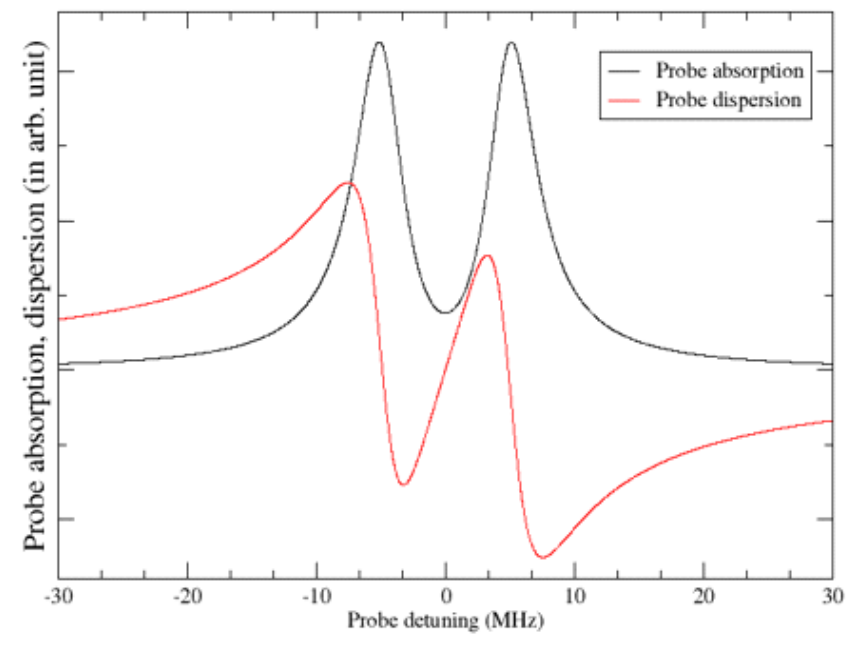

Figure 7: Variation of probe absorption [Im $\left.\left(\sigma_{12}\right)\right]$, probe dispersion [Re $\left.\left(\sigma_{12}\right)\right]$ w.r.t. probe detuning ( $\delta)$ for a $\Lambda$-type system

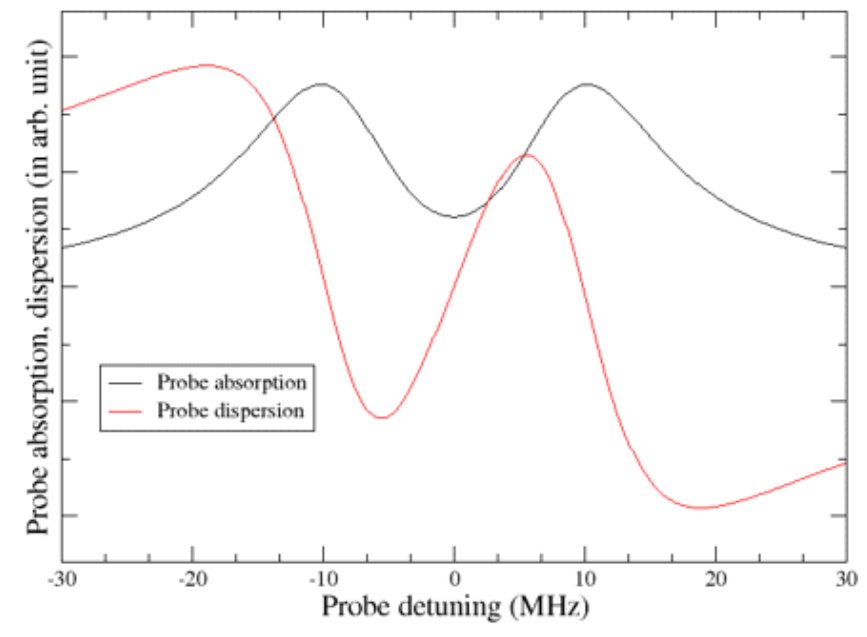

Figure 8: Variation of probe absorption [ $\left.\operatorname{Im}\left(\sigma_{12}\right)\right]$, probe dispersion $\left[\operatorname{Re}\left(\sigma_{12}\right)\right]$ w.r.t. probe detuning (ס) for a 三-type system 
The absorption and dispersion profiles of the probe field in the $\Lambda, \equiv$ and $V$-type systems are depicted in figures 7-9, respectively when pump detuning $=\Delta=0$, probe Rabi frequency $=\Omega P=2 \pi \times 1 \mathrm{MHz}$, pump Rabi frequency $=\Omega C=2 \pi \times 10 \mathrm{MHz}$. A pronounced transparency window at the line center is observed in each figure. It is evident that for $\Delta=\delta$ i.e., when the Raman resonance condition is satisfied the medium becomes transparent to the probe field. Also, the probe dispersion around $\delta=0$ is anomalous.

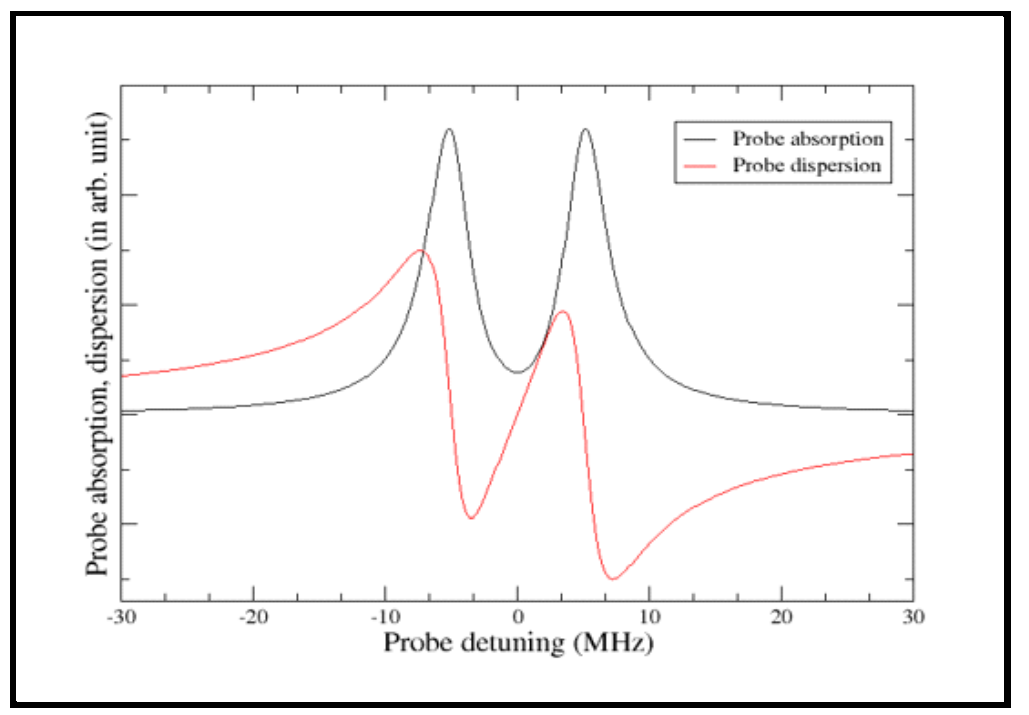

Figure 9: Variation of probe absorption $\left[\operatorname{Im}\left(\sigma_{13}\right)\right]$, probe dispersion $\left[\operatorname{Re}\left(\sigma_{13}\right)\right]$ w.r.t. probe detuning (ס) for a V-type system

\section{CONCLUSION}

The distinct features of EIT have gained extensive attentions of the researchers in the field of quantum optics. For three level systems as discussed in this article, one can notice a single EIT window in the absorption spectrum. At EIT resonance, the probe dispersion in the three-level atomic medium is anomalous. The full width at half maxima (FWHM) of the EIT dip depends on different factors such as the strength of the coupling or pump field, ground, or excited state decoherence rate etc. Here they have only examined the atomic configurations with hyperfine levels. Though, Zeeman sub-levels of the hyperfine energy levels can also be used. Using Zeeman sub-levels, the coherence manipulations can effectively be altered. While the atomic levels are increased, the light induced coherences in the system are modified vigorously. Naturally, the number of EIT windows as well as their appearance at the specific probe frequencies are also modified. Different other phenomena like Electromagnetically Induced Absorption $(E I A)$, lasing without population inversion (LWI), gain without population inversion $(\mathrm{GWI})$ are observed in the three as well as multilevel EIT atomic media. Thus, the profound impact of EIT technique upon the optical science has opened different ways to deal with the coherent preparation of atoms by $\mathrm{E} M$ fields. A well discussed topic in this context is the controlling of group velocity of light. The probe dispersion around the EIT window infers that the propagation of the probe field through the medium is subluminal or the group velocity of the light is slowed within the EIT window. Another application of the elimination of absorption by EIT is present in the non-linear optics. EIT has overcome many insurmountable obstacles in non-linear optics. Under EIT condition the Kerr non-linearity is enhanced and at the same time it gives rise to a stringent limit on the available single photon non-linear phase shift for pulsed lasers. Not only that, EIT has opened new ways in the quantum information processing, vector magnetometry, crossphase modulation, wave-mixing and so on. New applications continue to appear regularly by using the well-established EIT technique.

\section{ACKNOWLEDGEMENT}

The authors express their sincere thanks to Department of Biotechnology, Government of India for the funding from DBT-STAR College grant, under which this review project was conducted. They are also grateful to the Principal and the DBT-STAR Coordinator, Surendranath College, for their support and encouragement in implementing this review project at undergraduate level. 


\section{REFERENCE}

Boller, K. J., Imamoğlu, A., \& Harris, S. E. (1991). Observation of electromagnetically induced transparency. Physical Review Letters, 66(20), 2593.

Boon, J. R., Zekou, E., Fulton, D. J., \& Dunn, M. H. (1998). Experimental observation of a coherently induced transparency on a blue probe in a Doppler-broadened mismatched V-type system. Physical ReviewA, 57(2), 1323.

Eberly, J. H., Pons, M. L., \& Haq, H. R. (1994). Dressed-field pulses in an absorbing medium. Physical Review Letters, 72(1), 56.

Field, J. E., Hahn, K. H., \& Harris, S. E. (1991). Observation of electromagnetically induced transparency in collisionally broadened lead vapor. Physical Review Letters, 67(22), 3062.

Fulton, D. J., Shepherd, S., Moseley, R. R., Sinclair, B. D., \& Dunn, M. H. (1995). Continuous-wave electromagnetically induced transparency: A comparison of $\mathrm{V}, \Lambda$, and cascade systems. Physical ReviewA, 52(3), 2302.

Harris, S. E., Field, J. E., \& Imamoğlu, A. (1990). Nonlinear optical processes using electromagnetically induced transparency. Physical Review Letters, 64(10), 1107.

Hau, L. V., Harris, S. E., Dutton, Z., \& Behroozi, C. H. (1999). Light speed reduction to 17 metres per second in an ultracold atomic gas. Nature, 397(6720), 594-598.

Jin, S., Li, Y., \& Xiao, M. (1995). Hyperfine spectroscopy of highly excited atomic states based on atomic coherence. Optics Communications, 119(1-2), 90-96.

Kasapi, A. (1996). Enhanced isotope discrimination using electromagnetically induced transparency. Physical Review Letters, 77(6), 1035. 


\title{
Limitations of MOSFET Scaling and its Solution
}

\author{
Ishan Saha, Mohammad Javed Imam, Bonsari Choudhury, Haridas Kanji, Budhaditya Das, \\ Emona Datta* \\ Department of Physics, Surendranath College, Kolkata, India
}

*Corresponding Author's Email: edsnc123@gmail.com

\begin{abstract}
Over the past decades, scaling of complementary metal-oxide-semiconductor (CMOS) technology is a main factor for constant improvement in semiconductor industry. However, because of downscaling, when the device dimensions reduce to nanometer range, CMOS devices face lots of severe problems. Some of them are increase in leakage currents, complexity to elevate on-current, low reliability and increment in manufacturing cost etc. To maintain the improvements, various materials and advanced device structures had been introduced.
\end{abstract}

Keywords: Short-Channel Effect; Junctionless Transistor(JLT); Tunnel FET(TFET)

\section{INTRODUCTION}

For the past few decades, the complementary metal-oxide-semiconductor (CMOS) has been scaled down following Moore's Law to produce improved performance. According to Moore's Law, the number of transistors in an integrated circuit doubles approximately in every eighteen months and the rate will continue (Moore, 1965). Moore's observation also suggested that the size of individual components should be reduced so that more components can be included in an integrated circuit, which will further reduce the cost per functionality. Following this suggestion, MOSFET is being scaled down till date to meet the increasing demand of market. For the last three decades, this IC industry has been constantly scaling down the device dimension parameters, decreasing the chip size as well as improving the device and circuit design. As a result, this industry is benefitting from the exponential increase in chip speed and functionality density along with a decrease in power dissipation. However, the device dimension must be scaled down following a proper guideline. A fundamental method has been proposed by Dennard et al. in 1974, known as constant-field scaling. In this method, the device dimensions, doping concentration and supply voltage are scaled simultaneously in such a way that the electric field does not change. According to this, various device dimensions (both vertical and horizontal) such as, device width (W), gate-length (L), oxide thickness ( $t$ ), junction depth $(X)$ and supply voltage $(V D D)$
are downscaled by a factor $\alpha$ ( $(>1)$. As a result of rigorous downscaling, the device some problems that are yet to be solved. Increase in leakage current prevents further reduction in threshold voltage, which creates obstacles in reduction of supply voltage. Also, generated electric field and high doping concentration cause several challenging problems which worsen device performances.

To reduce these problems in CMOS devices, various prospects have been adopted. A few examples are multi-gate structures for better gate control over channel, using higher mobility channel materials for better on current, strain engineering etc. Also, alternative device structure has been proposed by various researchers to continue Moore's Law.

In this article, problems faced due to scaling device dimensions which hold backs further scaling down of metal-oxide-semiconductor field-effect transistors (MOSFETs) are discussed. After these issues are analyzed, various techniques to reduce those problems have been investigated.

\section{LITERATURE REVIEW}

\section{Challenges of MOSFET scaling:}

Leakage current: The current conduction mechanism in MOSFET involves thermionic emission of 
electrons over an energy barrier which sets a fundamental limit to subthreshold swing (S). In subthreshold region, the amount of gate voltage required in a transistor to increase the drain current by a magnitude of one order is known as subthreshold swing.

$$
\begin{aligned}
& S=\frac{d V_{G}}{d \Psi_{S}} \frac{d \Psi_{S}}{d\left(\log _{10} I_{D}\right)} \cong\left(1+\frac{C_{d}}{C_{o x}}\right) \ln 10 \frac{k T}{q} \\
\rightarrow & \frac{k T}{q} \ln 10 \cong 60 \mathrm{mV} \mathrm{decade}
\end{aligned}
$$

Where $I_{D}$ is the drain current, $k T / q$ is the thermal voltage, $V_{G}$ is the gate voltage, and $C_{o x}$ and $C_{d}$ are the oxide and depletion capacitances, respectively. This equation shows that with decrease in gate voltage, the transistor can be turned off abruptly. As gate length is reduced, supply voltage $V_{D D}$ as well as threshold voltage $V_{T H}$ also decrease to keep the overdrive voltage $\left(V_{D D}-V T H\right)$ high. But the reduction in $V_{T H}$ increases leakage current exponentially due to non-scalable $S$ which has a physical limit of $60 \mathrm{mV} /$ decade at room temperature. Typical values of $S$ are found to be around $100 \mathrm{mV} /$ decade for advanced CMOS; while keeping overdrive voltage high by lowering $\mathrm{V}_{\mathrm{DD}}$ from $500 \mathrm{mV}$ to $250 \mathrm{mV}$, it also has been observed that leakage current increases by a factor of 275 in bulk CMOS technology (Kim et al. 2009).

\section{Controlling power consumption:}

The power consumption for CMOS circuits can be categorized in two parts, dynamic and static. The dynamic power consumption is defined as the energy required for switching the inverter from one state to other through repeatedly charging and discharging the load capacitor $\left(C_{L}\right)$ connected to the inverteroutput. If supply voltage $V_{D D}$ is applied to the CMOS inverter which is operating at a frequency $f$, the dynamic power consumed can be given by:

$$
P_{\text {dynamic }}=C_{L} V_{D D}^{2} f
$$

On the other hand, static power consumption can be expressed as:

$$
P_{\text {static }}=I_{\text {leak }} V_{D D}
$$

So, the total power consumed by each inverter is:

$$
P_{\text {total }}=P_{\text {dynamic }}+P_{\text {static }}
$$

Maximum operating frequency directs to increased dynamic power dissipation which can be reduced by lowering $\mathrm{V}_{\mathrm{DD}}$. But decrease in supply voltage will worsen drive current which, in turn, increases inverter delay. Therefore, to maintain the speed of operation, $\mathrm{V}_{\mathrm{TH}}$ should be scaled down together with $\mathrm{V}_{\mathrm{DD}}$ to keep overdrive voltage high. But with the reduction in $\mathrm{V}_{\mathrm{TH}}$, leakage current increases exponentially for which static power increases. It has been observed that although dynamic power consumption decreases with reduction in $\mathrm{V}_{\mathrm{D}}$, static power component increases and after certain limit it exceeds dynamic component. As total power consumed depends on both dynamic and static power components, it increases with scaling of $V_{D D}$.

\section{Short-Channel effects:}

A MOSFET is small if the channel width is of same order of the depletion width. In this situation, the potential distribution depends on both transverse and longitudinal field due to gate and drain bias respectively. This results in many undesirable effects in MOSFET. These are known as Short-channel effects.

i. Carrier mobility degradation: Since the reduction in power supply does not occur in proportion to channel length, the electric field keeps increasing inside MOSFET. For lower values of field $\left(<10^{3} \mathrm{~V} / \mathrm{cm}\right)$, the carrier drift velocity is proportional to electric field across the channel. However, after that point, drift velocity tends to decrease with increase in field. Finally, the velocity saturates at higher values of field. This is known as "Velocity Saturation", originates due to various scattering mechanism (Pierret, 1996; Anderson \& Anderson, 2004). Another reason for high electric field is aggressive gate oxide thickness scaling, which confines the carriers in a smaller region at oxide/channel interface which leads to scattering of carriers and hence degradation in mobility. 
ii. Hot carrier effects: The high electric field causes various reliability challenges such as threshold voltage shift and degradation in trans-conductance because of Hot-carrier effects. As a result of increased scattering at high electric field, the drift velocity of the carriers begins to saturate, and it gains kinetic energy. At conduction band, electrons (carriers) have potential energy only, as it gains more kinetic energy, it moves higher up in conduction band. A few of the electrons can become energetic enough to cross the energy barrier between channel and gate oxide. Some of them can cross gate oxide and collected as gate current which in turn reduces input impedance. Some of the carriers can be trapped in gate oxide which increases $\mathrm{V}_{\mathrm{TH}}$.

iii. Punch-through: Punch-through is an extreme case of barrier lowering. With the increase in $V_{D D}$, the depletion width around drain can broaden further towards the source with depletion regions merging. This is known as "Punch-through". For this, gate losses its control over channel increasing drain current $\left(I_{D}\right)$ sharply. These effects increase with decrease in channel length.

iv. Drain-induced barrier lowering (DIBL): Reduction in $V_{T H}$ at higher values of drain voltage is referred as DIBL. When $\mathrm{V}_{G S}$ (gate voltage) $<\mathrm{V}_{T H}$, the surface cannot be inverted, carriers in channel faces potential barrier which blocks any flow. By increasing gate voltage, this barrier can be reduced. For short channel devices, this barrier is controlled by both $V_{G S}$ and $V_{D S}$ (drain voltage). If $V_{D S}$ is increased, channel potential barrier is reduced leading to current flow between source and drain, even at VGS < $\mathrm{V}_{\mathrm{TH}}$. The concept of channel barrier lowering for drain bias and reduced $\mathrm{V}_{\mathrm{TH}}$, is called DIBL.

\section{DISCUSSION}

To further extend Moore's law, various research has proposed alternative CMOS-compatible devices. Some of them have been discussed here. Due to the scaling process, device dimension has become so small that ultrasharp doping concentration gradients are required in junctions. This formation of ultrasharp doping requires costly millisecond annealing techniques, and it imposes several limitations on process thermal budget. To remove this problem of doping, a transistor that has no junction from source to drain has been proposed (Colinge et al. 2010a), (Rudenko et al.. 2012), (Kranti et al., 2010). In $\mathrm{JLT}$, the doping concentration in the channel is the same as that of the source and drain. As the doping concentration gradient is zero, no diffusion can occur which eliminates the necessity of ultrafast annealing technique. The main advantages of JLT over its conventional counterpart are as follows: better short channel immunity (Lee et al., 2010a), lower electric field in the ON-state (Colinge et al., 2010b), improved drain-induced barrier lowering (DIBL) (Lee et al., 2010b) etc.

In MOSFET, thermionic injection of electrons causes a fundamental limit to subthreshold swing. As tunnel FET (TFET) can overcome the fundamental subthreshold swing (SS) limitation of a conventional device, it is being considered as a promising successor of conventional metal-oxide- semiconductor FET (MOSFET) in recent years (Reddick \& Amaratunga, 1995). TFET has various advantages over conventional MOFET: (i) Due to lower S, TFET is suitable for low power application; (ii) TFET does not follow the traditional scaling rules like MOSFET to keep the same electric field throughout the device; (iii) as the channel region for TFET is intrinsic, the $\mathrm{V}_{\mathrm{TH}}$ fluctuations can be suppressed.

Following Moore's law, the Si-based CMOS technology has entered the nanoscale regime. Since then, conventional Si CMOS technology has faced several major challenges due to rigorous scaling of device dimensions. Even though some of the problems have already been dealt with, e.g., 3-D FinFET architecture, using high- $k$ gate dielectrics, etc., several are yet to be solved. To increase the drive current for improved performance is one of them. In the past few years, various materials have been aggressively investigated as alternative channel materials to Si such as Ge for p-channel and III-V compound semiconductors for $n$-channel transistors. It is revealed that saturation velocity and electron mobility of InGaAs are two times and ten times greater than that of $\mathrm{Si}$, respectively (Yonai et al., 2011; Kato et al., 2013).

\section{CONCLUSION}

As the scaling of device has reached its fundamental limit, various researchers have proposed alternative way to continue Moore's law. This technology cycle is being slowed down due to several 
problems like increment in power consumption, limitations on fabrication process, short channel effects etc. So, to reduce those problems and to give improved performance at low power, emerging devices have been considered for further use.

\section{ACKNOWLEDGEMENT}

The authors express their sincere thanks to Department of Biotechnology, Government of India for the funding from DBT-STAR College grant, under which this review project was conducted. We are also grateful to the Principal and the DBT-STAR Coordinator, Surendranath College, for their support and encouragement in implementing this review project at undergraduate level.

\section{REFERENCE}

Anderson, B., \& Anderson, R. (2004). Fundamentals of Semiconductor Devices. McGraw-Hill, Inc.

Colinge, J. P., Lee, C. W., Afzalian, A., Akhavan, N. D., Yan, R., Ferain, I., .. \& Murphy, R. (2010a). Nanowire transistors without junctions. Nature Nanotechnology, 5(3), 225-229.

Colinge, J. P., Lee, C. W., Ferain, I., Akhavan, N. D., Yan, R., Razavi, P., ... \& Doria, R. T. (2010b). Reduced electric field in junctionless transistors. Applied Physics Letters, 96(7), 073510.

Dennard, R. H., Gaensslen, F. H., Yu, H. N., Rideout, V. L., Bassous, E., \& LeBlanc, A. R. (1974). Design of ion-implanted MOSFET's with very small physical dimensions. IEEE Journal of Solid-State Circuits, 9(5), 256-268.

Kato, A., Kanazawa, T., Uehara, E., Yonai, Y., \& Miyamoto, Y. (2013, May). Sub-50-nm InGaAs MOSFET with n-InP source on Si substrate. In 2013 International Conference on Indium Phosphide and Related Materials (IPRM) (pp. 1-2). IEEE.

Kim, D., Lee, Y., Cai, J., Lauer, I., Chang, L., Koester, S. J., ... \& Blaauw, D. (2009). Heterojunction tunneling transistor (HETT)-based extremely low power applications. In Proc. Int. Symp. Low Power Electron. Design (pp. 219-224).

Kranti, A., Yan, R., Lee, C. W., Ferain, I., Yu, R., Akhavan, N. D., ... \& Colinge, J. P. (2010, September). Junctionless nanowire transistor (JNT): Properties and design guidelines. In 2010 Proceedings of the European Solid State Device Research Conference (pp. 357-360). IEEE.

Lee, C. W., Ferain, I., Afzalian, A., Yan, R., Akhavan, N. D., Razavi, P., \& Colinge, J. P. (2010b). Performance estimation of junctionless multigate transistors. Solid-State Electronics, 54(2), 97-103.

Lee, C. W., Nazarov, A. N., Ferain, I., Akhavan, N. D., Yan, R., Razavi, P., ... \& Colinge, J. P. (2010a). Low subthreshold slope in junctionless multigate transistors. Applied Physics Letters, 96(10), 102106.

Moore, G. E. (1965). Cramming more components onto integrated circuits. Electronics, 38(8), 114-117.

Pierret, R. F. (1996). Semiconductor Device Fundamentals. Pearson Education India.

Reddick, W. M., \& Amaratunga, G. A. (1995). Silicon surface tunnel transistor. Applied Physics Letters, 67(4), 494-496.

Rudenko, T., Nazarov, A., Ferain, I., Das, S., Yu, R., Barraud, S., \& Razavi, P. (2012). Mobility enhancement effect in heavily doped junctionless nanowire silicon-on-insulator metal-oxidesemiconductor field-effect transistors. Applied Physics Letters, 101(21), 213502.

Yonai, Y., Kanazawa, T., Ikeda, S., \& Miyamoto, Y. (2011, December). High drain current (> 2A/mm) InGaAs channel MOSFET at $\mathrm{V} D=0.5 \mathrm{~V}$ with shrinkage of channel length by $\mathrm{InP}$ anisotropic etching. In 2011 International Electron Devices Meeting (pp. 13-3). IEEE. 


\title{
A Comprehensive Study of the Past, Present and Future of Solid-state Drive
}

\author{
Subhrangshu Hait, Shib Shankar Biswas* \\ Department of Physics, Surendranath College, Kolkata, India \\ ${ }^{*}$ Corresponding Author's Email: Shibphy@gmail.com
}

\begin{abstract}
Scientists and engineers have been putting their efforts for years to prepare data storage more concise, speedy, reputable, less power consumption and accessible. Because of changes in the world new technology has seek remarkable revolution in it and one of them is flash memory which is known as its renowned application the solid-state disk or solid state derive (SSD). This paper enumerates the writings the development of SSD in brief.
\end{abstract}

\section{Keywords: Solid State Drive (SSD); Hard Disk Drive (HDD); Flash Storage Medium}

\section{INTRODUCTION}

Technology plays an important role in a changing world and the world changes with the change in technology. In the past data was saved to floppy disks or external drives because the internal hard disk drives used in computers in the market at that time were very expensive. The standard computer came to the market in 1980 and installed internal hard disk drives which were of megabytes order i.e., much less than the hard disks we are now using. But the biggest difficulty at that time is that if floppy disks and internal hard drives are found to be a problem, it is very difficult to recover the data stored there and sometimes the stored data is lost. This led to some other thinking about the alternative to floppy disks. CDs, DVDs, Blu-ray drives are used as alternatives to floppy drives between 1990 and 2000. As result Storage capacity also increases and in 2010 Tera Bytes drive is published. At this time, USB flash drive and SD memory card as new levels medium debuted in the market. SD memory card and USB flash drive developed new hard drive known as solid state drive (SSD). Solid State Drive is ahead of hard drive in the combinations of low random-access time, comparatively pretty read speed, less energy consumption and higher vibration tolerance which brings about fascinating choice in numerous applications.

\section{LITERATURE REVIEW}

\section{Solid state drives-a brief history}

The journey of SSD started with two new technology developments they are magnetic core memory (Eckert, 1997) and charged capacitor read-only storage. But these did not last long after the debut of drum storage it was cancelled because they were very expensive later in the 1970s and 1980 technology changed rapidly and the SSD drastically changed due to semiconductor memory improvement in the archaic Cray, IBM, and Amdahl supercomputer. Although people used them very little for high price.

Then in the year 1970 a renowned computer company, Dataram, launched the bulk core which had capacity $2 \mathrm{mb}$ and played a very effective role in digital equipment. Then in 1978, a different, the Texas memory system, produced superior competence SSD (16kb Ram SSD) and RAM SSD developed storage Tek Company in this year also.

Later in 1983, a 128kb SSD debuted with bubble memory. Then high-tech SSD series upgrades were made with the Tallgrass technologies corporation $20 \mathrm{MB}$ unit, and it is suitable for hard drive that is used in computer. In 1989, the Psion MC mobile computers used solid state disk with four slot memory storage 430. In 1991, SanDisk corporation launched $20 \mathrm{mb}$ solid state drive and the price of it was one thousand Dollar. In 1995 the M- system flashed based solid-state drive (Birrell et al. 2007) and its memory does not require any battery for data minutes but not as fast as dram. It is considered at the time of birth of modern SSD. 
Since 1995, various types of improvements have been made. In 2000, SSD first came to the market with the rise of netbook. In 2007, the OLPC XO-1 used 1GB SSD, the Asus Eee PC also utilised 2GB as elementary STROGE. As the netbook increases, the SSD capacity increases. In 2013 HGST released the first 12gb SSD drive. In the same year Western Digital launched the World's First Dual Drive with SSD And HDD Drive (refer to figure 1).

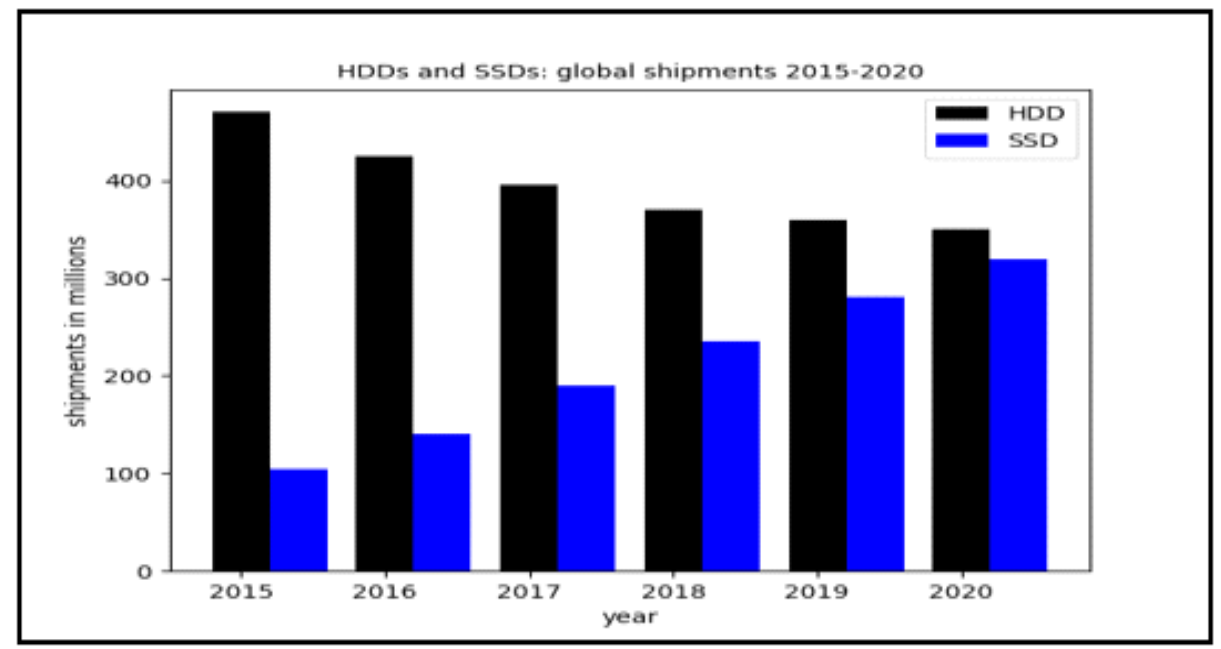

Figure 1: HDDs and SSDs: Global Shipments 2015-2020

In recent year SSD have become highly regarded and contains a good selection of application. This graph shows shipment numbers for solid state drives and hard drives throughout the world during the period 2015 to 2020.

\section{Major features and working procedure}

Several features characterize the design of an SSD. Solid State drives use flash memory (Chiang, Lee \& Chang, 1997) to provide higher performance and durability. In addition, SSD power consumption is low, compared to HDD light weight, resulting in laptop and mobile le SSD fittings easily. In addition, SSD controller software contains predictive analytic that sends us warning messages of potential drive faults. SSD can be purchased in different sizes $2.5 \mathrm{inch}, 1.8 \mathrm{inch}, 1 \mathrm{inch}$, for all these reasons most of the consumer consider SSD as a primary drive using his or her operating system and most important programs.

To explain the working procedure of SSD (Mao, Wu \& Duan, 2017), foremost it is required to bore into the internal function of normal HDD. An HDD consists of spinning disks coated by an electromagnetic component. There is a notch in the record player a needle to explain the data, the HDD got magnetic charges that narrate either 1 or 0 (Binary) which an arm reads to maze together the data stored. This is one of the most significant demerits of a normal HDD; with its moving parts it's subject to sensitivity from physical shocks and movement.

On the other side an SSD functions is the same manner alike RAM (random Access Memory or flash memory). The SSD saves data on chips in lieu of disks and it is non-volatile memory, which suggests it holds its data even if it loses power. Solid state drives utilise semiconductor chips to store data. In SSD cannot be overwritten. He has to be deleted from the file. Even if someone wants to delete any data from Windows or Mac operating systems, it doesn't get deleted instantly - the space is indicated as available for re-use. To truly re-use this space, the SSD has to opt for a "TRIM" command. After deleting adequate pages SSD will start a "garbage collection" or block. SSDs have more space that what's published in the advertisement thanks to over provisioning. Over provisioning is storage that's not included in the package but in lieu of that used for internal activity. The over-provisioned space occupied the minimum percentage of the solid-state drive. When no data is deleted block remapping occurs at the $70 \%$ mark. Solid state drive revolves all files in a cycle resulting the drive to close. Wear levelling is done at the end, it is a process of design which extends the lifetime of a solid-state drive. It prepares data so the erase cycles get disbursed equally all over the blocks of the device. 


\section{DISCUSSION}

\section{Future and challenges for SSD}

At present the future of the SSD is very bright. This drive has made global marketing a special place by leaving HDD behind for good enough performance. Although most of PC owner are converting their slots to hybrid drivers. Hybrid driver prices are low and offer very advanced data retrieval.

The solid-state drive avails flash storage medium, which is produced with NAND gates (Micheloni, Crippa \& Marelli, 2010) placed asynchronous, this design provides better storage density and reduces the cost compared to NOR gates Solid state memory devices like flash drives are hardware, which essentially means they're doing not require constant power to retain the knowledge. Flash drives draw power from your computer after they're plugged in and want no additional power supply. Memory cards, like compact flash and SD cards, are almost like flash drives, except they are doing not have the built-in USB connector to directly plug into your computer.

Regarding storage sanitization (Garfinkel \& Shelat, 2003) there are many Govt. standards as provided in its guidance. For shop, nevertheless, the standards are contrary of each other and issue direction for sanitizing computer storage in various manner. One because which creates difference of opinion among standards and the complication in sanitizing memory board is due to its internal design. The formation of memory and its procedure in SSDs are diverse from normal disk storage counterpart, which have been systemized over the preceding twenty years.

\section{CONCLUSION}

At last, SSDs are unthinkable devices that utilise non-volatile. Non-volatile storage producing devices carry out at very higher speed. There are various advantages of deploying SSD e.g., system making much speedier, making your computer more dependable and colling the system more efficiently. All these advantages are very important because it will impede the wastage to time \& money in future. In totality, everybody can be delighted from possessing an SSD. SSD is a tool which will be favourable to you irrespective of the fact that whether you are students, academic or staff.

\section{ACKNOWLEDGEMENT}

The authors express their cordial thanks to Department of Biotechnology, Government of India for financial support from DBT-STAR College grant, under which this review project was conducted. The authors are also grateful to the Principal and the DBT-STAR Coordinator, Surendranath College, for their support and encouragement in implementing this review project at undergraduate level.

\section{REFERENCES}

Birrell, A., Isard, M., Thacker, C., \& Wobber, T. (2007). A design for high-performance flash disks. ACM SIGOPS Operating Systems Review, 41(2), 88-93.

Chiang, M. L., Lee, P. C., \& Chang, R. C. (1997, December). Managing flash memory in personal communication devices. In ISCE'97. Proceedings of 1997 IEEE International Symposium on Consumer Electronics (Cat. No. 97TH8348) (pp. 177-182). IEEE.

Eckert, J. P. (1997). A survey of digital computer memory systems. Proceedings of the IEEE, 85(1), 184-197.

Garfinkel, S. L., \& Shelat, A. (2003). Remembrance of data passed: A study of disk sanitization practices. IEEE Security \& Privacy, 1(1), 17-27.

Mao, B., Wu, S., \& Duan, L. (2017). Improving the SSD performance by exploiting request characteristics and internal parallelism. IEEE Transactions on Computer-Aided Design of Integrated Circuits and Systems, 37(2), 472-484.

Micheloni, R., Crippa, L., \& Marelli, A. (2010). Inside NAND flash memories. Springer Science \& Business Media. 
Published by :

Lincoln Research and Publications Limited, Australia in collaboration with Lincoln University College, Malaysia

ISBN: 978-0-6488798-5-5

\section{www.lincolnrpl.org www.lucp.net}

